University of Nebraska - Lincoln

DigitalCommons@University of Nebraska - Lincoln

Catalog of the type specimens of Gelechioidea (Lepidoptera) in the collection of the National Museum of Natural History, Smithsonian Institution, Washington, DC

John W. Brown

Smithsonian Institution, Washington. DC

David Adamski

Smithsonian Institution, Washington. DC

Ronald W. Hodges

Smithsonian Institution, Washington. DC

Stephen M. Bahr II

Texas A\&M University

Follow this and additional works at: https://digitalcommons.unl.edu/systentomologyusda

Part of the Entomology Commons

Brown, John W.; Adamski, David; Hodges, Ronald W.; and Bahr, Stephen M. II, "Catalog of the type specimens of Gelechioidea (Lepidoptera) in the collection of the National Museum of Natural History, Smithsonian Institution, Washington, DC" (2004). USDA Systematic Entomology Laboratory. 62.

https://digitalcommons.unl.edu/systentomologyusda/62

This Article is brought to you for free and open access by the Entomology Collections, Miscellaneous at DigitalCommons@University of Nebraska - Lincoln. It has been accepted for inclusion in USDA Systematic Entomology Laboratory by an authorized administrator of DigitalCommons@University of Nebraska - Lincoln. 


\section{ZOOTAXA}

510

Catalog of the type specimens of Gelechioidea (Lepidoptera) in the collection of the National Museum of Natural History, Smithsonian Institution, Washington, DC

JOHN W. BROWN, DAVID ADAMSKI, RONALD W. HODGES \& STEPHEN M. BAHR, II

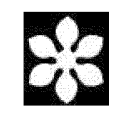

Magnolia Press

Auckland, New Zealand 
JOHN W. BROWN, DAVID ADAMSKI, RONALD W. HODGES \& STEPHEN M. BAHR, II Catalog of the type specimens of Gelechioidea (Lepidoptera) in the collection of the National Museum of Natural History, Smithsonian Institution, Washington, DC

(Zootaxa 510)

160 pp.; $30 \mathrm{~cm}$.

14 May 2004

ISBN 1-877354-40-6 (Paperback)

ISBN 1-877354-41-4 (Online edition)

FIRST PUBLISHED IN 2004 BY

Magnolia Press

P.O. Box 41383

Auckland 1030

New Zealand

e-mail: zootaxa@mapress.com

http://www.mapress.com/zootaxa/

(C) 2004 Magnolia Press

All rights reserved.

No part of this publication may be reproduced, stored, transmitted or disseminated, in any form, or by any means, without prior written permission from the publisher, to whom all requests to reproduce copyright material should be directed in writing.

This authorization does not extend to any other kind of copying, by any means, in any form, and for any purpose other than private research use.

This article is a U.S. government work, and is not subject to copyright in the United States.

ISSN 1175-5326 (Print edition)

ISSN 1175-5334 (Online edition) 


\title{
Catalog of the type specimens of Gelechioidea (Lepidoptera) in the collection of the National Museum of Natural History, Smithsonian Institution, Washington, DC
}

\author{
JOHN W. BROWN, DAVID ADAMSKI, RONALD W. HODGES \& STEPHEN M. \\ BAHR, II \\ (JWB, DA, RWH) Systematic Entomology Laboratory, PSI, Agricultural Research Service, U. S. Department of \\ Agriculture, clo National Museum of Natural History, Smithsonian Institution, Washington, DC 20013-7012, \\ USA (e-mail: jbrown@sel.barc.usda.gov); (SMB) Entomology Department, Texas A\&M University, College \\ Station, Texas, 77843, USA
}

\section{Table of contents}

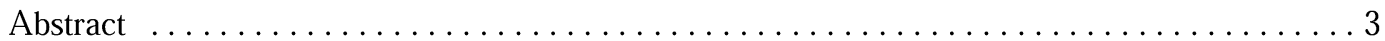

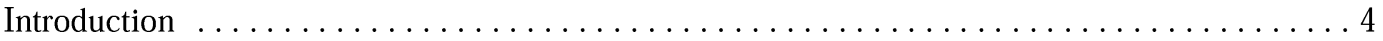

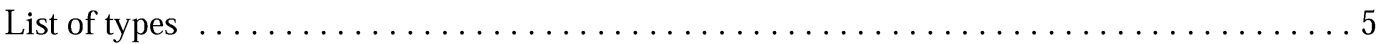

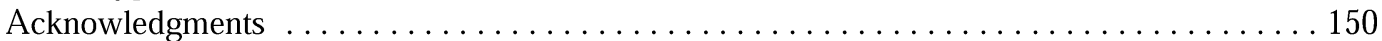

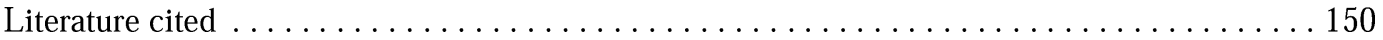

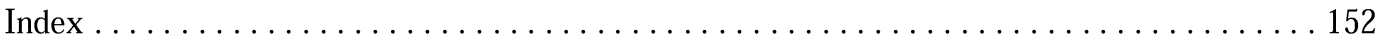

\begin{abstract}
The collection of the National Museum of Natural History, Smithsonian Institution, Washington, D.C., is second only to that of The Natural History Museum (formerly British Museum of Natural History), London, in the number of type specimens of the superfamily Gelechioidea (Lepidoptera). The Smithsonian houses 1,375 gelechioid types: 1,249 holotypes, 48 lectotypes, 1 neotype, 69 species represented by one or more syntypes, and 8 species represented by one or more "pseudotypes" (i.e., specimens identified as "type" by an accompanying label that are unlikely to be the type). Three former curators are responsible for the vast majority of the type specimens: August Busck, J. F. Gates Clarke, and Ronald W. Hodges. We present a list of the species for which a type is deposited in the USNM, organized alphabetically. For each species we provide an abbreviated reference to the original description and label data. This list represents the second contribution to a larger effort to make available information on the Lepidoptera type holdings of the USNM.
\end{abstract}

Key words: Lepidoptera, Gelechioidea, type specimens, catalogue, USNM 


\section{Introduction}

The type collection of Gelechioidea in the National Museum of Natural History, Smithsonian Institution (USNM) is second in number of types only to that of The Natural History Museum, London, England, and represents a significant resource for researchers interested in the taxonomy, phylogeny, biology, biodiversity, and conservation of gelechioid moths (Lepidoptera: Gelechioidea) worldwide. The strengths of the collection, reflecting primarily the field work of former curators rather than donations or accessions from abroad, lie in the faunas of North and South America and Oceania. The foundation of the type collection is represented by the large number of species (about 430) described by August Busck, the foremost North American lepidopterist of his time, who was employed by the U.S. Department of Agriculture (USDA) and located at the USNM from about 1900 to 1940 (Heinrich \& Loftin 1944). J. F. G. (Jack) Clarke, whose work spans the period from about 1935-1985, was employed by USDA early in his career and later by the Smithsonian Institution. Clarke was as comfortable in the field as he was in the museum, and his field work took him to many exotic places near and far. His monographic treatments of various gelechioid taxa, especially Oecophoridae, added substantially to the USNM gelechioid type holdings. From about 1962-1997, Ronald W. Hodges was USDA's microlepidopterist at the USNM, and the thrust of his systematic research focused on Gelechioidea, primarily the North American fauna, epitomized by several volumes in the series The Moths of America North of Mexico (1974, 1978, 1986, 1999). Details on the history of the Lepidoptera collection can be found in Flint et al. (1976).

Faunal treatments of the Philippine Islands (Diakonoff 1968), Rapa Island (Clarke 1971), Micronesia (Clarke 1976), Marquesas Archipelago (Clarke 1986), Palaeartic and Oriental Lecithoceridae (Park 1995, 1999, 2001; Park and Wu 2001, 2003; Wu and Park 1998, 1999; Wu 1998), and Elachistidae (Kaila 1995, 1996, 1997, 1999) resulted in the deposition of many gelechioid types in the USNM.

Recent curatorial efforts resulted in the organization of the type specimens of Gelechioidea into a single collection. The specimens are arranged alphabetically by species (or subspecies) in individual unit trays and stored in 29 insect drawers in the $5^{\text {th }}$ Floor, East Wing of the Natural History Building. The purpose of this work is to list the type specimens as part of a larger effort to make available information regarding the type holdings of USNM Lepidoptera to workers worldwide. This is the second major group, following the Tortricidae (Brown \& Lewis 2000), for which a comprehensive type catalog has been compiled for the USNM Lepidoptera types. Consistent with the collection, this list is arranged alphabetically by species, followed by author, year of publication, and genus (or genus and species for subspecies) in parentheses in which the taxon was originally described. References are spelled out in full. The sex, kind of type (e.g., holotype, lectotype, syntype), and label data also are included. We use the term "pseudotype" when the specimen is labeled as the type but there is convincing evidence to the contrary. Following 
the specimen data are the USNM type number and/or genitalia slide number, where applicable. For some species, remarks are provided on specimen condition or on ambiguities associated with the type(s). Current subfamily and/or family placement (Hodges 1998) for each taxon is provided in brackets at the end of each citation. We have attempted to provide the maximum collection data, relying on both the text of the original description and information on specimen labels. For example, country, state, county, province, etc. frequently are not present on the specimen labels; this information is provided where retrievable accurately. Abbreviations and symbols in the list include the following: $\mathrm{CNC}=$ Canadian National Collection, Ottawa, Ontario, Canada; BMNH = The Natural History Museum (formerly British Museum (Natural History)), London, United Kingdom; MCZ = Museum of Comparative Zoology, Harvard University, Cambridge, Massachusetts, USA; r.f. $=$ reared from; em. = emerged; $\mathrm{mi}=$ mile(s); no. = number; " = feet (elevation). Where the collecting data can have more than one interpretation, they are presented in quotes. For example, "Aug. 8/15" could be interpreted as "8 August 1915" or 8-15 August [no year].

At present (2004), the USNM gelechioid type collection includes 1,249 holotypes, 48 lectotypes, 1 neotype, 69 species represented by one or more syntypes, and 8 species represented by one or more pseudotypes. The following list includes all types putatively depo-sited in the USNM, regardless of whether we could locate them. In cases where we were unable to find the type, we annotate the species' entry with "Specimen not found." The fate of these specimens is uncertain, but they may be on loan, lost, or merely residing undiscovered in the main collection. The annotation "USNM Genitalia Slide No...." indicates that the slide is part of the "curated" USNM slide collection. Other references to slides (e.g., J. F. G. Clarke Slide No...) indicate the slide has not been located, although it is likely to be present in the USNM "pro-tem" slide collection. The genitalia of the type can be assumed to be undissected unless information to the contrary is provided (i.e., genitalia slide number or "abdomen lacking" annotation). In most cases where both the fore- and hindwings are missing from a specimen, it is likely that a wing slide has been made; however, we only present the slide number for those species with which we have associated the wing slide.

\section{List of types}

abactella Clarke, 1932 (Gelechia); Canadian Entomologist 64: 67. Holotype ․ Canada, British Columbia, Kaslo, [no date], Cockle. USNM Genitalia Slide No. 8703. [Gelechiidae: Gelechiinae]

abdominella Busck, 1903 (Gelechia); Proceedings of the U.S. National Museum 25: 863. Holotype 9 , USA, Arizona, Phoenix, August, Kunze. USNM Type No. 6378, USNM Genitalia Slide No. 8140. [Gelechiidae: Gelechiinae] 
abdominella Busck, 1912 (Ethmia); Proceedings of the Entomological Society of Washington 14: 85. Holotype ${ }^{\star}$, Mexico, Tehuacan, October 1910, R. Müller. USNM Type No. 14525. [Elachistidae: Ethmiinae]

abella Busck, 1903 (Gelechia); Proceedings of the U.S. National Museum 25: 889. Holotype 9 , USA, Colorado, Glenwood Springs [no date]. USNM Type No. 6392, USNM Genitalia Slide No. 7838. Left fore- and hindwing glued to double-mounted block. [Gelechiidae: Gelechiinae]

aberratella Busck, 1907 (Glyphidocera); Journal of the New York Entomological Society 15: 138. Holotype ${ }^{*}$, USA, Maryland, Montgomery Co., Plummers Island, Potomac River, July 1906, A. Busck. USNM Type No. 10322, USNM Genitalia Slide No. 10639. [Glyphidoceridae]

abludius Hodges, 1978 (Eralea); Moths of America north of Mexico, fasc. 6.1: 40. Holotype ${ }^{*}$, USA, Florida, Archbold Biological Station, Lake Placid, 23-31 May 1964, R. W. Hodges. USNM Type No. 70566, USNM Genitalia Slide No. 5583. [Cosmopterigidae: Cosmopteriginae]

absaroka Kaila, 1996 (Elachista); Entomologica Scandinavica 27: 223. Holotype o* USA, Wyoming, 6 mi NW Newcastle, 23 June 1965, R. W. Hodges. USNM Genitalia Slide No. 89275. [Elachistidae: Elachistinae]

acaciella Busck, 1900 (Polyhymno); Journal of the New York Entomological Society 8: 235. Holotype ${ }^{\star}$, USA, Texas, r.f. Acacia farnesiana, em. 17 April 1896. USNM Type No. 5353, Genitalia Slide AB Aug. 1, 1935. [Gelechiidae: Gelechiinae]

acaciella Busck, 1906 (Telphusa); Proceedings of the U.S. National Museum 30: 722. Holotype $\$$, USA, Texas, Brownsville, June 1904, H. S. Barber. USNM Type No. 9765, USNM Genitalia Slide No. 10652. [Gelechiidae: Gelechiinae]

acamtopappi Busck, 1915 (Coleophora); Proceedings of the Entomological Society of Washington 17: 87. Holotype (uncertain sex), USA, California, Los Angeles, r.f. Acamtopappus sphaerocephalus, October, A. Koebele. USNM Type No. 19240. Specimen not found. [Coleophoridae: Coleophorinae]

acanthocarpae Clarke, 1947 (Chionodes); Journal of the Washington Academy of Sciences 37: 248. Holotype o*, USA, Texas, Eagle Pass, 18 May 1938, L. F. Hitchcock. USNM Genitalia Slide No. 8124. [Gelechiidae: Gelechiinae] 
acanthovalva Park, 2001 (Hyperochtha); in Park \& Wu, Insecta Koreana 18: 140. Holotype $\diamond^{\star}$, Sri Lanka, Kan. District, Peradeniya, 2300', Upper Hantahe Hill, 12-16 January 1970, D. Davis \& B. Rowe. [Lecithoceridae]

acertella Busck, 1913 (Semioscopis); Journal of Entomology and Zoology (Pomona College) 5: 100. Holotype o*, USA, San Diego Co., San Diego, November, W. S. Wright. USNM Type No. 15613, J. F. G. Clarke Genitalia Slide No. 1236. [Cosmopterigidae: Antequerinae]

achne Hodges, 1986 (Dichomeris); Moths of America north of Mexico, fasc. 7.1: 97. Holotype $\sigma^{*}$ USA, Florida, Highlands Co., Parker Island, 26-29 May 1964, R. W. Hodges. USNM Genitalia Slide No. 9422. [Gelechiidae: Dichomeridinae]

achrantella Kaila, 1997 (Elachista); Acta Zoologica Fennica 206: 44. Holotype ơ, USA, Colorado, Alamosa Co., Sand Dunes Staff Quarters, 8200', pinyon-juniper zone, 27 June 1982, R. W. Hodges. USNM Genitalia Slide No. 89298. [Elachistidae: Elachistinae]

acmaea Clarke, 1978 (Lelita); Smithsonian Contributions to Zoology 273: 60. Holotype $q$, Chile, Llanquihue, Peulla, 9 March 1959, J. F. G. Clarke. USNM Type No. 73724, USNM Genitalia Slide No. 24203. Right forewing lacking. [Oecophoridae: Oecophorinae]

acompsa Walsingham, 1912 (Durrantia); Biologia Centrali-Americana, Lepidoptera Heterocera 4: 115. Holotype o*, Panama, Canal Zone, Tabernilla, June 1907, A. Busck. Abdomen lacking. [Peleopodidae]

acribostola Diakonoff, 1968 (Lecithocera); Bulletin of the U.S. National Museum 257 (1967): 139. Holotype ${ }^{*}$, Philippine Islands, Luzon, Mt. Makiling, [no date], P. J. Baker. USNM Genitalia Slide No. 89994. [Lecithoceridae]

acris Park, 1995 (Hypatima); Tropical Lepidoptera 6: 83. Holotype ${ }^{\star}$, Taiwan, Tainan Co., 2-3 km S Kwantzuling, ca. $350 \mathrm{~m}$, bamboo shrub, 26-28 June 1980, D. Davis. USNM Genitalia Slide No. 87401. The species name is misspelled on the type label. [Gelechiidae: Dichomeridinae]

acritomorpha Clarke, 1964 (Palinorsa); Proceedings of the U.S. National Museum 116: 203. Holotype ${ }^{\star}$, Peru, Tingo Maria, 24 November 1949, H. A. Allard. USNM Type No. 64992, USNM Genitalia Slide No. 25770. [Oecophoridae: Oecophorinae]

acritopterus Clarke, 1986 (Asymphorodes); Smithsonian Contributions to Zoology 416: 263. Holotype ${ }^{\star}$, Marquesas Archipelago, Fatu Hiva, Omoa, 17 March 1968, J. F. G. \& T. 
acronitis Busck, 1911 (Stenoma); Proceedings of the U.S. National Museum 40: 213. Holotype $0^{x}$, British Guiana, [no date], C. W. Bebee. USNM Type No. 13569, USNM Genitalia Slide No. 13079. [Elachistidae: Stenomatinae]

actiella Barnes \& Busck, 1920 (Isophrictis); Contributions to the Natural History of the Lepidoptera of North America 4: 224. Holotype ${ }^{*}$, USA, California, San Diego Co., San Diego, 24-30 May. USNM Genitalia Slide No. 5617. [Gelechiidae: Gelechiinae]

acuminae Adamski \& Brown, 2001 (Glyphidocera); Proceedings of the Entomological Society of Washington 103: 974. Holotype o*, Venezuela, Territorio Federal Amazona, Cerro de la Neblina, canopy, 155 m, 23-29 February 1984, D. Davis \& T. McCabe. USNM Genitalia Slide No. 81643. [Glyphidoceridae]

acycla Diakonoff, 1968 (Hypodrasia); Bulletin of the U.S. National Museum 257 (1967): 156. Holotype ${ }^{\star}$, Philippine Islands, Luzon, Los Baños, [no date], P. J. Baker. USNM Genitalia Slide No. 89976. [Gelechiidae: Dichomeridinae]

adam Hodges, 1999 (Chionodes); Moths of America north of Mexico, fasc. 7.6: 140. Holotype $\sigma^{x}$, USA, Arizona, Santa Cruz Co., Santa Rita Mountains, Madera Canyon, 22 May 1963, J. G. Franclemont. [Gelechiidae: Gelechiinae]

adamas Hodges, 1999 (Chionodes); Moths of America north of Mexico, fasc. 7.6: 150. Holotype $\sigma^{*}$, USA, Arkansas, Washington Co., Devil's Den State Park, 13 July 1966, R. W. Hodges. [Gelechiidae: Gelechiinae]

adamsi Hodges \& Adamski, 1997 (Filatima); Journal of the Lepidopterists' Society 51: 40. Holotype ${ }^{\star}$, USA, Maine, Little Wood Island, West Point, 13 August 1972, S. B. Adams. USNM Genitalia Slide No. 87529. [Gelechiidae: Gelechiinae]

addon Busck, 1911 (Stenoma); Proceedings of the U.S. National Museum 40: 221. Holotype ${ }^{*}$, British Guiana, Rockstone, Essequebo, [no date], W. Schaus. USNM Type No. 13585, USNM Genitalia Slide No. 12711. [Elachistidae: Stenomatinae]

adeneia Clarke, 1984 (Elaeonoma); Insects of Micronesia 9: 147. Holotype or, Micronesia, Eastern Caroline Island, Kusaie, Hill 541, 165 m, 20 March 1953, J. F. G. Clarke. USNM Type No. 76072. [Oecophoridae: Oecophorinae] 
adianta Kaila, 1997 (Elachista); Acta Zoologica Fennica 206: 39. Holotype ${ }^{\star}$, USA, Colorado, Alamosa Co., Sand Dunes Staff Quarters, 8200', 23 June 1982, pinyon/juniper zone, R.W. Hodges. USNM Genitalia Slide No. 89288. [Elachistidae: Elachistinae]

adustella Jäckh, 1978 (Scythris); Bolletino del Museo Civico di Storia Naturali 5: 1. Holotype $\sigma^{\star}$, Italy, Veneto Province, Verona, Mti Lessini, Cancello, 500 m, 24 August 1974. USNM Genitalia Slide No. 87774. According to the original description, deposited in Museo Civico Storia Naturale, Genova, Italy. [Xyloryctidae: Scythridinae]

adynatus Clarke, 1986 (Asymphorodes); Smithsonian Contributions to Zoology 416: 225. Holotype + , Marquesas Archipelago, Hiva Oa, Mt. Feani, 3400', 1 March 1968, J. F. G. \& T. Clarke. USNM Type No. 100790, USNM Genitalia Slide No. 24827. [Cosmopterigidae: Cosmopteriginae]

aenigma Clarke, 1986 (Asymphorodes); Smithsonian Contributions to Zoology 416: 255. Holotype , Marquesas Archipelago, Hiva Oa, Mt. Feani, 3400', 1 March 1968, J. F. G. \& T. Clarke. USNM Type No. 100811, USNM Genitalia Slide No. 25111. [Cosmopterigidae: Cosmopteriginae]

aenigmatica Clarke, 1962 (Trichotaphe); Proceedings of the Hawaiian Entomological Society 18: 123. Holotype $\sigma^{*}$, Mexico, Vera Cruz, Boca del Rio, r.f. Pluchea odorata, December 1960, N. L. H. Krauss. J. F. G. Clarke Genitalia Slide No. 10802. [Gelechiidae: Dichomeridinae]

aerinella Kaila, 1999 (Elachista); Acta Zoologica Fennica 211: 17. Holotype o , USA, Arizona, Coconino Co., 16 mi SW Flagstaff, West Fork, 6500', 13 July 1961, R. W. Hodges. USNM Genitalia Slide No. 10800. [Elachistidae: Elachistinae]

aethographa Clarke, 1971 (Gonionota); Smithsonian Contributions to Zoology 95: 4. Holotype ${ }^{\star}$, Costa Rica, Las Cruces, near San Vito, 19-20 March 1965, S. S. \& W. D. Duckworth. USNM Type No. 71084, USNM Genitalia Slide No. 68758. [Oecophoridae: Oecophorinae]

aethoptera Clarke, 1971 (Gonionota); Smithsonian Contributions to Zoology 95: 2. Holotype 9 , Venezuela, Aragua, Rancho Grande, 16-23 October 1966, S. S. \& W. D. Duckworth. USNM Type No. 71083, USNM Genitalia Slide No. 68759. [Oecophoridae: Oecophorinae]

affirmatella Busck, 1914 (Stenoma); Proceedings of the U.S. National Museum 47: 51. Holotype 9 , Panama, Canal Zone, June 1911, A. Busck. USNM Type No. 16737, USNM Genitalia Slide No. 13627. [Elachistidae: Stenomatinae]

GELECHIOIDEA TYPES IN USNM ～(C) 2004 Magnolia Press 

(1967): 129. Holotype 9 , Philippine Islands, Luzon, Mt. Makiling, [no date], P. J. Baker. USNM Genitalia Slide No. 89993. [Lecithoceridae]

aglaia Hodges, 1986 (Dichomeris); Moths of America north of Mexico, fasc. 7.1: 85. Holotype ${ }^{*}$, USA, Florida, Highlands Co., Archbold Biological Station, Lake Placid, 4 April 1959, R. W. Hodges. [Gelechiidae: Dichomeridinae]

agramma Becker, 1982 (Timocratica); Bulletin of the British Museum (Natural History), Entomology 45: 232. Holotype $\diamond^{\star}$, Brazil, Espirito Santo, [no date], F. Johnson. USNM Genitalia Slide No. 14026. [Elachistidae: Stenomatinae]

alabama Landry, 1994 (Coleophora); Canadian Entomologist 126: 1186. Holotype $\sigma^{*}$, USA, Alabama, Baldwin Co., Gulf State Park, 25 July 1985, R. L. \& B. B. Brown. USNM Genitalia Slide No. 87592. According to the original description, the holotype is deposited in the collection of the Mississippi Entomological Museum, Mississippi State University — on indefinite loan to USNM. [Coleophoridae: Coleophorinae]

alaricella Busck, 1908 (Gnorimoschema); Canadian Entomologist 40: 193. Holotype o*, USA, Pennsylvania, Allegheny Co., Oak Station, 2 September 1907, F. Marloff. USNM Type No. 11557, USNM Genitalia Slide No. 6912. [Gelechiidae: Gelechiinae]

albapalpella Chambers, 1881 (Elachista); Journal of the Cincinnati Society of Natural History 3: 294. Holotype 9 , USA, Massachusetts, Amherst, from Goodell. Genitalia Slide 23 Oct 1945, H.W.C. \#2. [Elachistidae: Elachistinae]

albapalpella Chambers, 1876 (Laverna); Cincinnati Quarterly Journal of Science 2 (1875): 295. Holotype + , USA, Colorado, Spanish Bar, 3 August. Specimen not found. [Coleophoridae: Momphinae]

albapenella Chambers, 1875 (Butalis); Canadian Entomologist 7: 11. Holotype o*, USA, Texas [no date]. USNM Genitalia Slide Nos. 81583, 81584. Specimen mounted on two microscope slides. [Coleophoridae: Blastobasinae]

albastrigulella Kearfott, 1907 (Dorata); Canadian Entomologist 39: 8. Holotype ๔, USA, California, Placer Co., 1 June 1904, A. H. Vachell. Specimen not found. [Oecophoridae: Oecophorinae]

albella Chambers, 1874 (Harpalyce); Canadian Entomologist 6: 235. Holotype (uncertain sex), USA, Texas [no date]. Two specimens of uncertain sex in the type collection, one with “Type” label. USNM Type No. 432. [Peleopodidae] 
albicapitella Engel, 1907 (Elachista); Entomological News 18: 277. Holotype $\sigma^{*}$, USA, Pennsylvania, Pittsburgh, 12-17 June, Engel. USNM Type No. 10285. Abdomen lacking. [Elachistidae: Elachistinae]

albicoma Clarke, 1986 (Asymphorodes); Smithsonian Contributions to Zoology 416: 211. Holotype $\sigma^{*}$, Marquesas Archipelago, Nuku Hiva, Tunoa Ridge, 2900', 23 January 1968, J. F. G. \& T. Clarke. USNM Type No. 100787, USNM Genitalia Slide No. 24723. [Cosmopterigidae: Cosmopteriginae]

albicornella Busck, 1914 (Walshia); Proceedings of the U.S. National Museum 47: 2. Holotype $\sigma^{*}$ Panama, La Chorrera, May 1912, A. Busck. USNM Type No. 15813. [Cosmopterigidae: Chrysopeleiinae]

albicostella Beutenmüller, 1889 (Psecadia); Entomologica Americana 5: 9. Holotype + , USA, Colorado, [no date], Beutenmüller. USNM Type No. 435. [Elachistidae: Ethmiinae]

albicostella Clarke, 1942 (Filatima); Proceedings of the U.S. National Museum 92: 272. Holotype $\diamond^{\star}$, USA, Washington, Pullman, 21 February 1935, J. F. G. Clarke. USNM Type No. 56273, USNM Genitalia Slide No. 8727. [Gelechiidae: Gelechiinae]

albicruris Park \& Heppner, 2000 (Torodora); Transactions of the Lepidopterological Society of Japan 51: 293. Holotype $\sigma^{*}$, Taiwan, Tainan Co., Konsirei (= Kuantzuling), 19 May 1934, S. Issiki. USNM Genitalia Slide No. 82492. [Lecithoceridae]

albifemorella Clarke, 1932 (Gelechia); Canadian Entomologist 64: 65. Holotype o*, USA, California, Mt. Shasta, 7000', 24-31 July, J. McDunnough. USNM Type No. 43517, USNM Genitalia Slide No. 9659. [Gelechiidae: Gelechiinae]

alboligula Hodges, 1962 (Anoncia); Entomologica Americana 42: 102. Holotype ${ }^{*}$, USA, California, Inyo Co., Olancha, 24-30 June. USNM Type No. 66087, USNM Genitalia Slide No. 4039. [Cosmopterigidae: Cosmopteriginae]

albomaculella Chambers, 1875 (Gelechia); Canadian Entomologist 7: 209. Holotype $\sigma^{*}$, Canada, Quebec, vicinity of Quebec City [no date]. USNM Type No. 5765. Left fore- and hindwing and abdomen lacking. [Gelechiidae: Gelechiinae]

aleatrix Hodges, 1986 (Dichomeris); Moths of America north of Mexico, fasc. 7.1: 91. Holotype $o^{*}$, USA, Illinois, Putnam Co., 15 June 1944, M. O. Glenn. USNM Genitalia Slide No. 9314. [Gelechiidae: Dichomeridinae] 
alexandrae Adamski \& Brown, 2001 (Glyphidocera); Proceedings of the Entomological Society of Washington 103: 984. Holotype $\sigma^{*}$, Venezuela, Territorio Federal Amazona, Cerro de la Neblina, base camp, $0^{\circ} 50^{\prime} \mathrm{N}, 66^{\circ} 9^{\prime} 4 " \mathrm{~W}, 155 \mathrm{~m}, 23-29$ February 1984, D. Davis \& T. McCabe. USNM Genitalia Slide No. 81640. [Glyphidoceridae]

alienella Busck, 1904 (Depressaria); Proceedings of the U.S. National Museum 27: 765. Holotype + , USA, Oregon, Josephine Co., Rogue River, r.f Artemisia sp.?, em. June 1872, Wlsm 90780. USNM Type No. 7816, USNM Genitalia Slide No. 547. In conflict with the data on the holotype, the original description states that the type "was taken at Kaslo, British Columbia, Canada, by Dr. Dyar." The holotype bears the same USNM type number referred to in the original description. Consequently, Busck may have erred on the locality. Hodges (1974) does not mention the discrepancy. [Elachistidae: Depressariinae]

allardi Clarke, 1951 (Coptotelia); Acta Zoologica Lilloana 11: 338. Holotype ox, Peru, Tingo Maria, 4 December 1949, H. A. Allard. USNM Type No. 61118, USNM Genitalia Slide No. 69424. [Oecophoridae: Oecophorinae]

alleriella Busck, 1940 (Aroga); Bulletin of the Southern California Academy of Sciences 39: 89. Holotype ${ }^{*}$, USA, Alabama, Mobile, r.f. Polygonum maritinum, 1 December 1924, T. Van Aller. USNM Type No. 54051, USNM Genitalia Slide No. 9904. [Gelechiidae: Gelechiinae]

alloea Walsingham, 1911 (Oestomorpha); Biologia Centrali-Americana, Lepidoptera Heterocera 4: 108. Holotype ơ, Mexico, Veracruz, Cordoba, May 1908, F. Knab. [Gelechiidae: Gelechiinae]

alnifructella Busck, 1915 (Recurvaria); Proceedings of the Entomological Society of Washington 17: 82. Holotype + , USA, Virginia [no date]. USNM Type No. 19226, USNM Genitalia Slide No. 6259. [Gelechiidae: Gelechiinae]

alternatella Kearfott, 1908 (Gelechia); Journal of the New York Entomological Society 16: 185. Holotype + , Canada, Manitoba, Aweme, 12-25 May, N. Criddle. R. W. Hodges Genitalia Slide No. 2555. [Gelechiidae: Gelechiinae]

alticolans Hodges, 1969 (Siskiwitia); Smithsonian Contributions to Zoology 18: 10. Holotype $\sigma^{\star}$, USA, Arizona, Santa Cruz Co., Santa Rita Mountains, Madera Canyon, Bog Springs Campground, 5100', 10-26 July 1964, D. R. Davis. [Cosmopterigidae: Chrysopeleiinae] 
altor Hodges, 1999 (Chionodes); Moths of America north of Mexico, fasc. 7.6: 141. Holotype ${ }^{*}$, USA, Arizona, Santa Cruz Co., Santa Rita Mountains, Madera Canyon, 14 June 1960, J. G. Franclemont. [Gelechiidae: Gelechiinae]

amauroptera Clarke 1971 (Gonionota); Smithsonian Contributions to Zoology 95: 13. Holotype ${ }^{*}$, Argentina, Tucumán, Ciudad Universitaria, Cerro San Javier, 800 m, 17 February 1959, J. F. G. Clarke. USNM Type No. 71090, USNM Genitalia Slide No. 68913. [Oecophoridae: Oecophorinae]

amblysoma Clarke, 1986 (Asymphorodes); Smithsonian Contributions to Zoology 416: 217. Holotype ${ }^{*}$, Marquesas Archipelago, Fatu Hiva, Tahuna, 2000', 27 March 1968, J. F. G. \& T. Clarke. USNM Type No. 100788, USNM Genitalia Slide No. 24758. [Cosmopterigidae: Cosmopteriginae]

amissella Busck, 1908 (Depressaria); Proceedings of the Entomological Society of Washington 9: 89. Holotype + , USA, Florida, Kissimmee, [no date], W. Beutenmüller. USNM Type No. 11326, USNM Genitalia Slide No. 281. Left forewing lacking. [Elachistidae: Depressariinae]

amphichroma Clarke, 1978 (Corita); Smithsonian Contributions to Zoology 273: 15. Holotype + , Chile, Centro-Austral, January-March 1898, V. Izquerdo. USNM Type No. 73696, USNM Genitalia Slide No. 24249. [Oecophoridae: Oecophorinae]

amrodella Kaila, 1999 (Elachista); Acta Zoologica Fennica 211: 71. Holotype ơ, USA, Colorado, Chaffee Co., Cottonwood Pass, 11600', ponderosa pine, pinyon-juniper cottonwood-aspen, 18 July 1982, R. W. Hodges. USNM Genitalia Slide No. 90198. [Elachistidae: Elachistinae]

amydrographa Diakonoff, 1968 (Tanyzancla); Bulletin of the U.S. National Museum 257 (1967): 171. Holotype \%, Philippine Islands, Luzon, Mt. Makiling, [no date], P. J. Baker. USNM Genitalia Slide No. 89978. [Oecophoridae: Oecophorinae]

amyrisella Busck, 1900 (Depressaria); Proceedings of the U.S. National Museum 23: 233. Holotype + , USA, Florida, Palm Beach, [no date], H. G. Dyar. USNM Type No. 4941, USNM Genitalia Slide No. 391. [Elachistidae: Depressariinae]

anagna Kaila, 1997 (Elachista); Acta Zoologica Fennica 206: 29. Holotype ơ, USA, Arizona, Coconino Co., 10 mi NNW Flagstaff, Hart Prairie, 8500', 1 July 1961, R. W. Hodges. USNM Genitalia Slide No. 89278. [Elachistidae: Elachistinae] 
analis Busck, 1914 (Cryptolechia); Proceedings of the U.S. National Museum 47: 30. Holotype ${ }^{\star}$, Panama, Porto Bello, April, A. Busck. USNM Type No. 16752, USNM Genitalia Slide No. 4080. [Oecophoridae: Oecophorinae]

ancylosana Wu \& Park, 1999 (Frisilia); Korean Journal of Systematic Zoology 15: 5. Holotype $\sigma^{*}$, Sri Lanka, Kan. District, Peradeniya, Upper Hantane Hill, 2300', $12-16$ January 1970, D. Davis \& B. Rowe. USNM Genitalia Slide No. 88982. [Lecithoceridae]

anelaea Meyrick, 1932 (Stenoma); Exotic Microlepidoptera 4: 305. Holotype o", Brazil, Ponte Nova, Rio Xingu [no date]. USNM Genitalia Slide No. 14023. Anterior part of abdomen in gelatin capsule. [Elachistidae: Stenomatinae]

angelicivora Clarke, 1952 (Depressaria); Smithsonian Miscellaneous Collections 117: 15. Holotype $\circ^{\star}$, USA, Montana, McDonald Pass, $14 \mathrm{mi}$ W Helena, 6100', r.f. Angelica arguta, em. 10 August 1950, J. F. G. Clarke. USNM Type No. 61133, USNM Genitalia Slide No. 583. [Elachistidae: Depressariinae]

angulata Park \& Hodges, 1995 (Dichomeris); Insecta Koreana 12: 43. Holotype o*, Taiwan, Nantou Co., Leinhauchi Forest Station, 15 km SW Puli, 750 m, 22-26 May 1980, D. Davis. USNM Genitalia Slide No. 87321. [Gelechiidae: Dichomeridinae]

angustalatella Powell, 1973 (Ethmia); Smithsonian Contributions to Zoology 120: 214. Holotype $o^{\star}$, Mexico, Nueva Leon, 3 mi E Galeana, 5000', 7-9 August 1963, W. D. Duckworth \& D. Davis. USNM Genitalia Slide No. 98450. [Elachistidae: Ethmiinae]

angustati Clarke, 1941 (Depressaria); Proceedings of the U.S. National Museum 90: 189. Holotype $\sigma^{*}$ USA, Washington, Whatcom Co., Skyline Ridge, Mount Baker District, 1718 August 1933, r.f. Lomatium angustatum, em. 31 August 1933, J. F. G. Clarke. USNM Type No. 52076, USNM Genitalia Slide No. 662. [Elachistidae: Depressariinae]

anita Busck, 1920 (Gonioterma); Insecutor Inscitiae Menstruus 8: 93. Holotype *, Guatemala, Cayuga, [no date], W. Schaus. USNM Type No. 22316, USNM Genitalia Slide No. 13339. [Elachistidae: Stenomatinae]

anna Busck, 1911 (Gonioterma); Proceedings of the U.S. National Museum 40: 224. Holotype 9 , Dutch Guiana, Geldersland, Surinam River, [no date], W. Schaus. USNM Type No. 13591, USNM Genitalia Slide No. 13290. [Elachistidae: Stenomatinae]

annulata Busck, 1914 (Perimede); Proceedings of the U.S. National Museum 47: 2. Holotype + , Panama, Canal Zone, Paraiso, May 1911, A. Busck. USNM Type No. 15814, USNM Genitalia Slide No. 8824. [Cosmopterigidae: Chrysopeleiinae] 
annulata Clarke, 1978 (Doina); Smithsonian Contributions to Zoology 273: 29. Holotype

USNM Genitalia Slide No. 24258. Right forewing lacking. [Amphisbatidae]

anomala Clarke, 1978 (Talitha); Smithsonian Contributions to Zoology 273: 10. Holotype 9. Chile, Santiago, Guatacan, 1100 m, October 1952, L. E. Peña. USNM Type No. 73693, USNM Genitalia Slide No. 24185. Right fore- and hindwing lacking. [Oecophoridae: Oecophorinae]

anomala Hodges, 1985 (Exotelia); Journal of the Lepidopterists' Society 39: 139. Holotype $\sigma^{*}$, USA, New Mexico, 40 km NE Silver City, r.f. Pinus ponderosa, June 1977, R. Stevens. [Gelechiidae: Gelechiinae]

anomalella Dietz, 1910 (Eubolepia); Transactions of the American Entomological Society 36: 68. Holotype 9 , USA, Arizona, Pinal Mountains [no date]. USNM Genitalia Slide No. 81032. [Coleophoridae: Blastobasinae]

anteliella Busck, 1903 (Paltodora); Proceedings of the U.S. National Museum 25: 778. Holotype ${ }^{*}$, USA, New Jersey, Montclair, [no date], W. D. Kearfott. USNM Type No. 6347, USNM Genitalia Slide No. 5618. [Gelechiidae: Gelechiinae]

antennariella Clarke, 1941 (Agonopterix); Proceedings of the U.S. National Museum 90: 108. Holotype $\sigma^{*}$, USA, Washington, Whitman Co., Kamiack Butte, 18 April 1935, r.f. Antennaria luzuloides, J. F. G. Clarke. USNM Type No. 52081. [Elachistidae: Depressariinae]

antichira Wu \& Park, 1999 (Timyra); Korean Journal of Systematic Zoology 15: 207. Holotype $\sigma^{\star}$, Sri Lanka, Tri. District, China Bay, 200', 9-11 November 1976, Hevel, Dietz, Karunaratne \& Balasooriya. USNM Genitalia Slide No. 90086. [Lecithoceridae]

aphidiella Walsingham, 1907 (Holcocera); Proceedings of the U.S. National Museum 33: 205. Holotype ${ }^{*}$, USA, Missouri, r.f. gall of Phylloxera on hickory, October 1882. USNM Type No. 10676, USNM Genitalia Slide No. 80993. [Coleophoridae: Blastobasinae]

apicalis Busck, 1911 (Stenoma); Proceedings of the U.S. National Museum 40: 215. Holotype $\sigma^{*}$, French Guiana, St. Jean, Maroni River, [no date], W. Schaus. USNM Type No. 13573, USNM Genitalia Slide No. 13162. [Elachistidae: Stenomatinae]

apicalis Diakonoff, 1968 (Odites); Bulletin of the U.S. National Museum 257 (1967): 122. Holotype $\sigma^{*}$, Philippine Islands, Luzon, Mt. Makiling, [no date], P. J. Baker. USNM Genitalia Slide No. 89991. [Lecithoceridae] 
apicistrigella Chambers, 1872 (Parasia); Canadian Entomologist 4: 66. Holotype o* USA, Kentucky [no date]. USNM Type No. 5780. Abdomen lacking. [Gelechiidae: Gelechiinae]

aporema Clarke, 1987 (Asymphorodes); Proceedings of the Biological Society of Washington 100: 596. Holotype ơ, Guam, 15 March 1939, R. G. Oakley. USNM Genitalia Slide No. 26411. [Cosmopterigidae: Cosmopteriginae]

aporia Clarke, 1986 (Asymphorodes); Smithsonian Contributions to Zoology 416: 201. Holotype $\sigma^{*}$, Marquesas Archipelago, Nuku Hiva, Tapuaooa, 30 January 1968, J. F. G. \& T. M. Clarke. USNM Type No. 100781, USNM Genitalia Slide No. 24940. [Cosmopterigidae: Cosmopteriginae]

arcanella Busck, 1912 (Dolidiria); Smithsonian Miscellaneous Collections 59: 5. Syntypes $\left(4{ }^{\circ}\right)$, Panama, Cabima and Trinidad River, June 1911, A. Busck. USNM Type No. 14531, USNM Genitalia Slide No. 86885, USNM Wing Slide No. 87127. One specimen dissected, two with abdomen lacking. [Peleopodidae]

arda Clarke, 1978 (Perzelia); Smithsonian Contributions to Zoology 273: 14. Holotype ox, Chile, Maule, Pelluhue, 600 m, 2 December 1953, L. E. Peña. USNM Type No. 73695. [Oecophoridae: Oecophorinae]

ardelia Hodges, 1986 (Dichomeris); Moths of America north of Mexico, fasc. 7.1: 62. Holotype $\diamond^{\star}$, USA, Florida, Archbold Biological Station, Lake Placid, 1-7 May 1964, R. W. Hodges. USNM Genitalia Slide No. 9138. [Gelechiidae: Dichomeridinae]

ardesiella Walsingham, 1911 (Dichomeris); Biologia Centrali-Americana, Lepidoptera Heterocera 4: 96. Holotype $o^{*}$, Mexico, Veracruz, Cordoba, May 1908, F. Knab, Walsingham No. 5765. [Gelechiidae: Dichomeridinae]

arena Kaila, 1997 (Elachista); Acta Zoologica Fennica 206: 15. Holotype o*, USA, Nevada, Lander Co., Toiyabe National Forest, Bob Scott Campground, 7300-7500', 3 June 1974, J. F. G. Clarke. USNM Genitalia Slide No. 89291. [Elachistidae: Elachistinae]

argentea Busck, 1914 (Pavolechia); Proceedings of the U.S. National Museum 47: 21. Holotype + , Panama, Trinidad River, March 1912, A. Busck. USNM Type No. 16679, USNM Genitalia Slide No. 10667, USNM Wing Slide No. 86541. [Gelechiidae: Gelechiinae] 
argentea Clarke, 1986 (Acanthophlebia); Smithsonian Contributions to Zoology 416: 312. Holotype $o^{\star}$, Marquesas Archipelago, Fatu Hiva, Omoa, 11 March 1968, J. F. G. \& T. M. Clarke. USNM Type No. 100828, USNM Genitalia Slide No. 25139. Left fore- and hindwing lacking. [Cosmopterigidae: Cosmopteriginae]

argentidisca Dognin, 1905 (Borkhausenia); Annales de la Société Entomologique de Belgique 49: 90. Holotype + , Brazil, São Paulo, Paranapanema [no date]. USNM Genitalia Slide No. 69552. [Oecophoridae: Oecophorinae]

argentidorsella Busck, 1911 (Hasta); Proceedings of the U.S. National Museum 40: 210. Holotype ${ }^{*}$, Brazil, Paraná, Castro, [no date], W. Schaus. USNM Type No. 13564, USNM Genitalia Slide No. 69378. [Elachistidae: Depressariinae]

argentifera Busck, 1903 (Aristotelia); Proceedings of the U.S. National Museum 25: 800. Holotype ${ }^{\star}$, USA, California, San Francisco Co., October, Koebele. USNM Type No. 6550, Genitalia Slide AB May 10, 1933. [Gelechiidae: Gelechiinae]

argentimaculella Murtfeldt, 1900 (Laverna); Canadian Entomologist 32: 161. Syntypes (uncertain sex), USA, Missouri, leaf-miner on Oenothera biennis, 1895. Several specimens in USNM reared on Oenothera by Murtfeldt; two specimens, both lacking the abdomen, are double-mounted together and bear a handwritten label "Type! AB[usck], from (?) M[issouri]," “371 M, mines in leaves of Oenothera, 10/4-11 [18]95." [Coleophoridae: Momphinae]

argentipunctella Ely, 1947 (Gelechia); Proceedings of the Entomological Society of Washington 12: 70. Holotype (uncertain sex), USA, Connecticut, East River, 7-12 July 1902, C. Ely. USNM Type No. 12882. Type consists of a portion of the thorax, the left midleg, and left forewing only. [Gelechiidae: Gelechiinae]

argilla Clarke, 1978 (Osmarina); Smithsonian Contributions to Zoology 273: 67. Holotype $\sigma^{*}$, Chile, Centro-Austral, January-March 1898, V. Izquerdo. USNM Type No. 73727. [Oecophoridae: Oecophorinae]

argopleura Clarke, 1971 (Gonionota); Smithsonian Contributions to Zoology 95: 12. Holotype 9 , Brazil, Santa Catarina, 26 September 1934, F. Plaumann. USNM Type No. 71089, USNM Genitalia Slide No. 68908. [Oecophoridae: Oecophorinae]

argutiola Hodges, 1974 (Aroga); Canadian Entomologist 106: 987. Holotype o*, USA, Michigan, Alcona Co., r.f. sweet fern, em. 24 May 1971, L. F. Wilson. USNM Genitalia Slide 2904. [Gelechiidae: Gelechiinae] 
argyreodema Wu \& Park, 1999 (Doxogenes); Korean Journal of Systematic Zoology 15: 215. Holotype ${ }^{*}$ Sri Lanka, NE District, Eliya, 8280', Mt. Pidurutalagala, 14 February 1970, D. Davis \& B. Rowe. USNM Genitalia Slide No. 89060. [Lecithoceridae]

argyrosticha Clarke, 1971 (Stathmopoda); Smithsonian Contributions to Zoology 56: 180. Holotype $o^{*}$, Rapa Island, Maugaoa, 950', 23 November 1963, J. F. G. \& T. M. Clarke. USNM Type No. 70084, USNM Genitalia Slide No. 26336. [Oecophoridae: Stathmopodinae]

argyrothamniella Busck, 1900 (Anacampsis); Proceedings of the U.S. National Museum 235: 231. Holotype $\sigma^{*}$, USA, Florida, Palm Beach, r.f. Argyrothamnia blodgettii, [no date], H. Dyar. USNM Type No. 4938, USNM Genitalia Slide No. 10654. [Gelechiidae: Gelechiinae]

arista Diakonoff, 1968 (Thymiatris); Bulletin of the U.S. National Museum 257 (1967): 112. Holotype $\sigma^{\star}$, Philippine Islands, Luzon, Los Baños, [no date], P. J. Baker. USNM Genitalia Slide No. 89984 [Xyloryctidae: Xyloryctinae]

aristella Busck, 1903 (Gelechia); Proceedings of the U.S. National Museum 25: 866. Lectotype ${ }^{\star}$, USA, Arizona, Williams, 17 July, H. S. Barber. USNM Type No. 6381, USNM Genitalia Slide No. 7860. Designated by Hodges (1999). [Gelechiidae: Gelechiinae]

arizonella Busck, 1903 (Gelechia); Proceedings of the U.S. National Museum 25: 856. Syntypes $\left(20^{\star}, 1^{\circ}\right)$, USA, Arizona, Santa Rita Mountains, 21 May 1898, 4 June, E. A. Schwarz. USNM Genitalia Slide No. 8749. [Gelechiidae: Gelechiinae]

armata Clarke, 1952 (Depressaria); Smithsonian Miscellaneous Collections 117: 19. Holotype ${ }^{*}$, USA, Washington, Whatcom Co., 6500', r.f. Lomatium brandegei, 4 August 1950, J. F. G. Clarke. USNM Type No. 61135, USNM Genitalia Slide No. 669. [Elachistidae: Depressariinae]

arnicella Clarke, 1942 (Gnorimoschema); Proceedings of the U.S. National Museum 92: 268. Holotype +, USA, Washington, Whitman Co., Kamiack Butte, 3000', 19 May 1934, J. F. G. Clarke. USNM Type No. 56268, USNM Genitalia Slide No. 6920. [Gelechiidae: Gelechiinae]

arsa Hodges, 1978 (Periploca); Moths of America north of Mexico, fasc. 6.1: 103. Holotype $\sigma^{\star}$, USA, Florida, Monroe Co., Key Largo, 17 October 1968, S. Kemp. USNM Genitalia Slide No. 4881. [Cosmopterigidae: Chrysopeleiinae] 
artemisiella Kearfott, 1903 (Gnorimoschema); Journal of the New York Entomological Society 11: 160. Holotype ${ }^{\star}$, USA, Illinois, Chicago, July, J. H. Reading. USNM Type No. 6816, USNM Genitalia Slide No. 648. According to the original description, this species was described from 24 specimens reared from Artemisia canadensis by Reading in Chicago. Kearfott clearly indicates “Type U.S. Nat. Mus.” [Gelechiidae: Gelechiinae]

aruns Hodges, 1999 (Chionodes); Moths of America north of Mexico, fasc. 7.6: 189. Holotype $\sigma^{*}$ USA, Texas, Culberson Co., Sierra Diablo, 20 mi NNW Van Horn, 6000', 27 May 1973, R. W. Hodges. [Gelechiidae: Gelechiinae]

ascriptella Busck, 1908 (Borkhausenia); Canadian Entomologist 40: 194. Lectotype o USA, Pennsylvania, Allegheny Co., Oak Station, 9 July 1907, F. Marloff. Abdomen lacking. Designated by Hodges (1974). [Oecophoridae: Oecophorinae]

asema Clarke, 1947 (Chionodes); Journal of the Washington Academy of Sciences 37: 243. Holotype ${ }^{\star}$, USA, Illinois, Putnam Co., 29 June 1941, M. O. Glenn. USNM Type No. 58234, USNM Genitalia Slide No. 9896. [Gelechiidae: Gelechiinae]

asemanta Dognin, 1905 (Cryptolechia); Annales de la Société Entomologique de Belgique 49: 88. Holotype ${ }^{\star}$, Ecuador, environs de Loja, Durrant "12.6.12." USNM Type No. 32358, USNM Genitalia Slide No. 4085. [Elachistidae: Depressariinae]

aspera Park, 1995 (Anarsia); Tropical Lepidoptera 6: 57. Holotype ${ }^{\star}$, Taiwan, Orchid (=Lanyu) Island, 4 km SW Hungta, 16-20 July 1980, D. Davis. USNM Genitalia Slide No. 87365. [Gelechiidae: Gelechiinae]

asperula Clarke, 1978 (Doina); Smithsonian Contributions to Zoology 273: 26. Holotype 9. Chile, Maule, Constitución, 26 November 1953, L. E. Peña. USNM Type No. 73703, USNM Genitalia Slide No. 24193. [Amphisbatidae]

atascosa Landry, 1991 (Arotrura); Entomological Society of Canada, Memoir 160: 62. Holotype $o^{\star}$, USA, Texas, Cameron Co., Laguna Atascosa, 11 November 1979, A. \& M. E. Blanchard. USNM Genitalia Slide No. 87226. [Xyloryctidae: Scythridinae]

atricastana Park, 1999 (Lecithocera); Zoological Studies 38: 254. Holotype ox, Taiwan, Taichung Co., Heiganzan (=Hungshan), 11 August 1943, A. Mutuura. USNM Genitalia Slide No. 82493. [Lecithoceridae]

atrimersa Diakonoff, 1968 (Odites perissa); Bulletin of the U.S. National Museum 257 (1967): 122. Holotype ${ }^{*}$, Philippine Islands, Luzon, Mt. Makiling, [no date], P. J. Baker. Specimen not found. [Lecithoceridae] 
atriplex Busck, 1910 (Gnorimoschema); Proceedings of the Entomological Society of Washington 11: 175. Holotype 9 , USA, California, San Bernardino Co., May, r.f. Atriplex canescens, A. Koebele. USNM Type No. 12700, USNM Genitalia Slide No. 7229. [Gelechiidae: Gelechiinae]

atriplicivora Cockerell, 1898 (Coleophora); Annals of Natural History 7: 403. Syntypes (uncertain sex), USA, New Mexico, Mesilla Park, r.f. cases on Atriplex canescens [no date]. Two cases on a single insect pin; no adults found. [Coleophoridae: Coleophorinae]

atrostrigella Clarke, 1941 (Depressaria); Proceedings of the U.S. National Museum 90: 168. Holotype ${ }^{\prime}$, Canada, Manitoba, Aweme, 6 September 1928, N. Criddle. USNM Type No. 53299, J. F. G. Clarke Genitalia Slide No. 2253. According to the original description, deposited in CNC. [Elachistidae: Depressariinae]

attenuata Clarke, 1986 (Pitycona); Smithsonian Contributions to Zoology 416: 175. Holotype + , Marquesas Archipelago, Fatu Hiva, Omoa, 13 March 1968, J. F. G. \& T. M. Clarke. USNM Type No. 100772, USNM Genitalia Slide No. 24575. [Gelechiidae: Gelechiinae]

atteria Busck, 1914 (Ancipita); Proceedings of the U.S. National Museum 47: 26. Holotype 9 , Panama, Cabima, June 1911, A. Busck. USNM Type No. 16688, USNM Genitalia Slide No. 69202. [Oecophoridae: Oecophorinae]

augusti Heinrich, 1920 (Holcocera); Proceedings of the U.S. National Museum 57: 70. Holotype $\sigma^{\star}$, USA, Oregon, Ashland, reared “15/1915,” J. E. Patterson. USNM Type No. 21811, Genitalia Slide CH-13, Dec. 1917. [Coleophoridae: Blastobasinae]

aulaea Clarke, 1932 (Gelechia); Canadian Entomologist 64: 68. Holotype *, USA, Washington, Pullman, 17 January 1931, T. C. Clarke. USNM Type No. 43452. [Gelechiidae: Gelechiinae]

aurea Busck, 1914 (Hamadera); Proceedings of the U.S. National Museum 47: 23. Holotype + , Panama, Trinidad River, March 1912, A. Busck. USNM Type No. 16682. Right fore- and hindwing lacking. [Oecophoridae: Oecophorinae]

auriciliella Busck, 1914 (Fortinea); Proceedings of the U.S. National Museum 47: 3. Holotype ${ }^{\star}$, Panama, Porto Bello, March 1911, A. Busck. USNM Type No. 15815, J. F. G. Clarke Genitalia Slide No. 10977. Right fore- and hindwing lacking. [Gelechiidae: Gelechiinae] 
auricincta Diakonoff, 1968 (Tisis); Bulletin of the U.S. National Museum 257 (1967): 125. Holotype $\sigma^{\star}$, Philippine Islands, Mindanao, Davao Province, east slope of Mount McKinley, 3000', September 1946, H. Hoogstraal. USNM Genitalia Slide No. 69000. [Lecithoceridae]

aurorella Dyar, 1902 (Semioscopis); Canadian Entomologist 34: 319. Syntype o* USA, Pennsylvania, New Brighton, 21 March 1902, H. D. Merrick. USNM Type No. 6610, USNM Genitalia Slide No. 491. The species was described from 4 syntypes; we were able to located only one. [Elachistidae: Depressariinae]

baccharisella Busck, 1903 (Gnorimoschema); Proceedings of the U.S. National Museum 25: 825. Syntype ${ }^{*}$, USA, California, Alameda Co., September, r.f. Baccharis pilularis. USNM Type No. 6359. Abdomen lacking. [Gelechiidae: Gelechiinae]

badia Hodges, 1962 (Sathrobrota); Entomologica Americana 42: 76. Holotype 9 , USA, South Florida, r.f. pine cones infested by Dioryctria, em. 16 June 1931, J. K. Small. USNM Type No. 66083. Abdomen lacking. [Cosmopterigidae: Cosmopteriginae]

baibarensis Park, 1999 (Homaloxestis); Zoological Studies 38: 240. Holotype o*, Taiwan, Taichung Co., Baibara, Meiyuan, 28 March 1943, S. Issiki. USNM Genitalia Slide No. 82495. [Lecithoceridae]

balbidophora Diakonoff, 1968 (Diploclasis); Bulletin of the U.S. National Museum 257 (1967): 168. Holotype $\sigma^{*}$, Philippine Islands, Luzon, Los Baños, [no date], P. J. Baker. USNM Genitalia Slide No. 89981, USNM Wing Slide No. 89982. [Oecophoridae: Oecophorinae]

baldella Barnes \& Busck, 1920 (Coelopoeta); Contributions to the Natural History of the Lepidoptera of North America 4: 248. Holotype o*, USA, California, San Bernardino Mts., Camp Baldy, 24-30 June. USNM Genitalia Slide No. 87422. [Oecophoridae: Oecophorinae]

baldiana Barnes \& Busck, 1920 (Telphusa); Contributions to the Natural History of the Lepidoptera of North America 4: 225. Syntypes $\left(1 \sigma^{*}, 2 \%\right)$, USA, California, San Bernardino, Camp Baldy, 1-7 June. USNM Type No. 6348, USNM Genitalia Slide Nos. 6645, 6648, 6649. [Gelechiidae: Gelechiinae]

baliostola Walsingham, 1912 (Ethmia); Biologia Centrali-Americana, Lepidoptera Heterocera 4: 144. Holotype ${ }^{\star}$, Costa Rica, Banana River, March 1906. USNM Type No. 68203, USNM Genitalia Slide No. 98454. [Elachistidae: Ethmiinae] 

type $\sigma^{\star}$, USA, Arizona, Palmerlee [no date]. USNM Genitalia Slide No. 1050. [Elachistidae: Depressariinae]

banksiella Busck, 1903 (Gnorimoschema); Proceedings of the U.S. National Museum 25: 832. Syntypes $\left(2{ }^{\star}\right)$, USA, New York, Long Island, Sea Cliff [no date]. USNM Type No. 6364. Abdomen lacking from both specimens. [Gelechiidae: Gelechiinae]

barberella Busck, 1902 (Depressaria); Proceedings of the U.S. National Museum 24: 747. Holotype ơ, USA, Arizona, Williams, [no date], H. S. Barber. USNM Type No. 6128. [Elachistidae: Depressariinae]

barberella Busck, 1903 (Neodactylota); Proceedings of the U.S. National Museum 25: 836. Holotype + , USA, Arizona, Williams [no date]. USNM Type No. 6362, USNM Genitalia Slide No. 7563. [Gelechiidae: Gelechiinae]

bardus Hodges, 1999 (Chionodes); Moths of America north of Mexico, fasc. 7.6: 99. Holotype $\sigma^{*}$, USA, California, Santa Barbara Co., Santa Barbara Island, Channel Islands National Park, 20 July 1987, r.f. Eriogonum giganteum. USNM Genitalia Slide No. 87591. [Gelechiidae: Gelechiinae]

barnesiella Busck, 1903 (Gelechia); Proceedings of the U.S. National Museum 25: 875. Syntype $\sigma^{*}$, USA, Colorado, Glenwood Springs, 1892. USNM Type No. 6386, USNM Genitalia Slide No. 7572. [Gelechiidae: Gelechiinae]

barnesiella Busck, 1907 (Trichotaphe); Proceedings of the Entomological Society of Washington 8: 92. Holotype ${ }^{\star}$, USA, Arizona, Redington [no date]. USNM Type No. 9897, USNM Genitalia Slide No. 10642. [Gelechiidae: Dichomeridinae]

basilaris Busck, 1914 (Stenoma); Proceedings of the U.S. National Museum 47: 45. Holotype $\sigma^{*}$, Panama, Trinidad River, March 1912, A. Busck. USNM Type No. 16721, USNM Genitalia Slide No. 12721. [Elachistidae: Stenomatinae]

basipallidella Dietz, 1910 (Holcocera dives); Transactions of the American Entomological Society 36: 27. Lectotype o*, USA, Massachusetts, Cohasset, [no date], O. Bryant. USNM Genitalia Slide No. 81004. Designated by Adamski \& Hodges (1996). [Coleophoridae: Blastobasinae]

basipunctella Adamski \& Brown, 2001 (Glyphidocera); Proceedings of the Entomological Society of Washington 103: 981. Holotype $\diamond^{\star}$, Venezuela, Territorio Federal Amazona, Cerro de la Neblina, base camp, $140 \mathrm{~m}, 0^{\circ} 50^{\prime} \mathrm{N}, 66^{\circ} 9^{\prime} \mathrm{W}, 24$ November-1 December 1984, R. Brown. USNM Genitalia Slide No. 81460. [Glyphidoceridae] 
basirosella Busck, 1914 (Costoma); Proceedings of the U.S. National Museum 47: 24.

Holotype ?, Panama, Trinidad River, June 1912, A. Busck. USNM Type No. 16683, USNM Genitalia Slide No. 69386. [Oecophoridae: Oecophorinae]

batanella Busck, 1903 (Gnorimoschema); Proceedings of the U.S. National Museum 25: 833. Syntype $\sigma^{\star}$, USA, New Jersey, Essex Co. Park, 17 June, W. Kearfott. USNM Type No. 6365, USNM Genitalia Slide No. 6926. [Gelechiidae: Gelechiinae]

baucidis Hodges, 1969 (Sorhagenia); Smithsonian Contributions to Zoology 18: 9. Holotype ${ }^{\star}$, USA, Illinois, Putnam Co., 17 June 1965, M. O. Glenn. USNM Type No. 69784, USNM Genitalia Slide No. 8824. Right forewing lacking. [Cosmopterigidae: Chrysopeleiinae]

baxa Hodges, 1986 (Dichomeris); Moths of America north of Mexico, fasc. 7.1: 105. Holotype $\diamond^{\star}$, USA, California, Monterey Co., Presidio of Monterey, 18 April 1944, r.f. Corythrogyne filaginifolia, J. F. G. Clarke. USNM Genitalia Slide No. 9387. [Gelechiidae: Dichomeridinae]

belangerella Chambers, 1875 (Gelechia); Canadian Entomologist 7: 210. Holotype o*, Canada [no other data]. Type represented by the head, thorax, and right wings only. USNM Type No. 5767. [Gelechiidae: Gelechiinae]

bella Duckworth, 1966 (Falculina); Proceedings of the U.S. National Museum 118: 398. Holotype ${ }^{\star}$, Brazil, above Obidos, Amazon, 10 September 1920, Cornell Univ. Exped. Lot 607, Sub 182. USNM Genitalia Slide No. 14124. [Elachistidae: Stenomatinae]

benitella Barnes \& Busck, 1920 (Gelechia); Contributions to the Natural History of the Lepidoptera of North America 4: 229. Holotype + , USA, Texas, San Benito, “Aug 8-15.” USNM Genitalia Slide No. 6743. Specimen lacking most appendages. [Gelechiidae: Gelechiinae]

bermudensis Hodges, 1966 (Batrachedra decoctor); Transactions of the American Entomological Society 92: 630. Holotype + , British West Indies, Bermuda, r.f. inflorescence of Serenoa repens, 16 May, F. M. Jones. USNM Type No. 67864, USNM Genitalia Slide No. 5161. [Batrachedridae]

besma Clarke, 1947 (Depressaria); Journal of the Washington Academy of Sciences 37: 14. Holotype $\sigma^{*}$, USA, Washington, Pierce Co., Fort Lewis, 30 May 1944, r.f. Lomatium utriculatum, J. F. G. Clarke. USNM Type No. 58010, USNM Genitalia Slide No. 634. [Elachistidae: Depressariinae] 
betina Clarke, 1947 (Depressaria); Journal of the Washington Academy of Sciences 37: 9.

Holotype ${ }^{*}$, USA, Washington, Klickitat Co., Gilmer, 2 June 1946, r.f. Lomatium triternatum macrocarpum, J. F. G. Clarke. USNM Type No. 58008, USNM Genitalia Slide No. 567. [Elachistidae: Depressariinae]

betulae Clarke, 1947 (Filatima); Journal of the Washington Academy of Sciences 37: 264. Holotype $\sigma^{\star}$, USA, Massachusetts, Melrose Highlands, r.f. Betula populifolia, 5 June 1931. USNM Type No. 58250, USNM Genitalia Slide No. 8763. [Gelechiidae: Gelechiinae]

betulella Busck, 1902 (Depressaria); Proceedings of the U.S. National Museum 24: 746. Holotype + , USA, Pennsylvania, r.f. Betula nigra, 1 July 1900, W. G. Dietz. USNM Type No. 6130, USNM Genitalia Slide No. 678. [Elachistidae: Depressariinae]

betulella Busck, 1903 (Telphusa); Proceedings of the U.S. National Museum 25: 787. Syntypes $\left(1 \sigma^{*}, 1^{\circ}\right)$, USA, Virginia, r.f. birch, em. 1 March 1884 and 23 July 1884 . USNM Type No. 6348, USNM Genitalia Slide No. 6648. [Gelechiidae: Gelechiinae]

bicolor Clarke, 1947 (Chionodes); Journal of the Washington Academy of Sciences 37: 250. Holotype ${ }^{\star}$, USA, California, Sonoma Co., Petaluma, 5-9 September 1936, E. C. Johnston. USNM Type No. 58240, USNM Genitalia Slide No. 9899. [Gelechiidae: Gelechiinae]

bicostomaculella Chambers, 1872 (Depressaria); Canadian Entomologist 4: 127. Neotype ‘, USA, Kentucky, "12/72.” USNM Genitalia Slide No. 7795. Left forewing lacking. Designated by Hodges (1999). [Gelechiidae: Gelechiinae]

bicristatella Chambers, 1875 (Gelechia); Canadian Entomologist 7: 210. Holotype $q$, Canada, Quebec [no date]. USNM Type No. 5764, Genitalia Slide RWH \# 725. Specimen comprised of head and part of thorax only. [Elachistidae: Agonoxeniinae]

bifasciella Busck, 1903 (Aristotelia); Proceedings of the U.S. National Museum 25: 799. Syntypes (4), USA, Arizona, Argus Mountains, April 1891, "through C. V. Riley." USNM Type No. 6349, Genitalia Slide AB May 10, 1931, USNM Genitalia Slide No. 5850. [Gelechiidae: Gelechiinae]

bifasciella Chambers, 1874 (Theisoa); Canadian Entomologist 6: 76. Holotype (uncertain sex), USA, Kentucky [no date]. [Gelechiidae: Gelechiinae]

biforella Busck, 1909 (Gelechia), Proceedings of the Entomological Society of Washington 11: 89. Holotype ${ }^{\circ}$, USA, Texas, Cotula, at light, 10 May 1906, J. C. Crawford. USNM Type No. 12263, USNM Genitalia Slide No. 8765. [Gelechiidae: Gelechiinae] 
bigella Busck, 1913 (Gelechia); Journal of Entomology and Zoology (Pomona College) 5: 96. Holotype ${ }^{\star}$, USA, California, San Diego, 19 February 1912, W. S. Wright. USNM

Type No. 15611, USNM Genitalia Slide No. 8768. [Gelechiidae: Gelechiinae]

biloba Hodges, 1978 (Afeda); Moths of America north of Mexico, fasc. 6.1: 127. Holotype \%, USA, Florida, Monroe Co., Tavernier, 6 October 1955, J. M. Todd. USNM Genitalia Slide No. 4877. [Cosmopterigidae: Chrysopeleiinae]

bimaculata Park, 1999 (Lecithocera); Zoological Studies 38: 244. Holotype ${ }^{\star}$, Taiwan, Taichung Co., Hassenzan (=Pahsienshan), 5 June 1942, S. Issiki. USNM Genitalia Slide No. 82494. [Lecithoceridae]

biminimaculella Chambers, 1880 (Gelechia); Journal of the Cincinnati Society of Natural History 2: 181. Syntypes $\left(2{ }^{\star}\right)$, USA, Texas [no date]. USNM Type No. 1525, USNM Genitalia Slide No. 9242. [Gelechiidae: Gelechiinae]

bipunctatus Clarke, 1986 (Asymphorodes); Smithsonian Contributions to Zoology 416: 232. Holotype $\sigma^{\star}$, Marquesas Archipelago, Nuku Hiva, Tapuaooa, 2500', 30 January 1968, J. F. G. \& T. M. Clarke. USNM Type No. 100794, USNM Genitalia Slide No. 24799. [Cosmopterigidae: Cosmopteriginae]

bipunctella Busck, 1906 (Numata); Proceedings of the U.S. National Museum 30: 724. Holotype $\sigma^{7}$, USA, Texas, Brownsville, June 1904, H. S. Barber. USNM Type No. 9767, USNM Genitalia Slide No. 10650. [Gelechiidae: Gelechiinae]

biscolorella Chambers, 1872 (Agnippe); Canadian Entomologist 4: 195. Holotype o*, USA, Kentucky, April. USNM Type No. 442, USNM Genitalia Slide No. 10680. Left fore- and hindwing lacking. [Gelechiidae: Gelechiinae]

bistrigella Busck, 1914 (Anacampsis); Proceedings of the U.S. National Museum 47: 9. Holotype $\sigma^{*}$, Panama, Alhajuela, May 1912, A. Busck. USNM Type No. 15822. [Gelechiidae: Gelechiinae]

bittenella Busck, 1906 (Tamarrha); Proceedings of the U.S. National Museum 32: 730. Holotype + , USA, Texas, Brownsville, in copula, 29 May 1904. USNM Type No. 9272. Right fore- and hindwing lacking. [Elachistidae: Ethmiinae]

blacella Barnes \& Busck, 1920 (Agonopteryx); Contributions to the Natural History of the Lepidoptera of North America 4: 232. Holotype 9 , USA, California, Truckee, 2 September 1913, X. McLashan. USNM Type No. 22668, USNM Genitalia Slide No. 288. [Elachistidae: Depressariinae] 
blanchardorum Hodges, 1986 (Dichomeris); Moths of America north of Mexico, fasc. 7.1: 43. Holotype $\sigma^{*}$, USA, Texas, Cameron Co., Laguna Atascosa, 22 November 1973, A. \& M. E. Blanchard. USNM Genitalia Slide No. 10975. [Gelechiidae: Dichomeridinae]

boleta Wu, 1998 (Tisis); Zoological Studies 37: 195. Holotype $\sigma^{\star}$, Indonesia, West Java, Mts. Gede-Panggrango, Tjibodas, 1400 m, 5 October 1966, J. F. G. \& T. M. Clarke. USNM Genitalia Slide No. 89132. [Lecithoceridae]

bolize Hodges, 1986 (Dichomeris); Moths of America north of Mexico, fasc. 7.1: 100. Holotype $\sigma^{*}$, USA, Nebraska, Cherry Co., Valentine NWR, Hackberry Lane, 15 June 1983, R. W. Hodges. USNM Genitalia Slide No. 12299. [Gelechiidae: Dichomeridinae]

bottimeri Busck, 1940 (Mompha); Bulletin of the Southern California Academy of Sciences 39: 87. Holotype $\sigma^{\star}$, USA, Florida, Alachua Co., Gainesville, 29 May 1937, r.f. seed capsules of Crocanthemum corumbosum, L. J. Bottimer. USNM Type No. 54052. [Coleophoridae: Momphinae]

bourquini Clarke, 1964 (Gonionota); Proceedings of the U.S. National Museum 115: 70. Holotype $\sigma^{\star}$, Brazil, New Bremen, 5 October, F. Plaumann. USNM Type No. 66371, USNM Genitalia Slide No. 68947. [Oecophoridae: Oecophorinae]

bovinella Busck, 1914 (Stenoma); Proceedings of the U.S. National Museum 47: 50. Holotype $\sigma^{\star}$, Panama, Canal Zone, June 1911, A. Busck. USNM Type No. 16736, USNM Genitalia Slide No. 13375. [Elachistidae: Stenomatinae]

braunae Clarke, 1941 (Semioscopis); Proceedings of the U.S. National Museum 90: 159. Holotype $\sigma^{x}$, USA, Ohio, Cincinnati, 29 March 1905, A. Braun. USNM Type No. 53118, USNM Genitalia Slide No. 476. [Elachistidae: Depressariinae]

bregorella Kaila, 1999 (Elachista); Acta Zoologica Fennica 211: 60. Holotype $\sigma^{*}$, USA, Arizona, Madera Canyon, Santa Rita Mts., 5600', 8 October 1959, R.W. Hodges. USNM Genitalia Slide No. 90106. [Elachistidae: Elachistinae]

brevimacula Clarke, 1986 (Asymphorodes); Smithsonian Contributions to Zoology 416: 203. Holotype $\sigma^{\star}$, Marquesas Archipelago, Hiva Oa, Mt. Feani, 3800', 30 February 1968, J. F. G. \& T. M. Clarke. USNM Type No. 100784, USNM Genitalia Slide No. 24824. [Cosmopterigidae: Cosmopteriginae]

brevisella Adamski \& Brown, 2001 (Glyphidocera); Proceedings of the Entomological Society of Washington 103: 987. Holotype $\sigma^{*}$, Venezuela, Territorio Federal Amazona, San Carlos de Rio Negro, $1^{\circ} 56^{\prime} \mathrm{N}, 67^{\circ} 02^{\prime} \mathrm{W}, 6-12$ December 1984, R. Brown. USNM Genitalia Slide No. 81634. [Glyphidoceridae] 
brevistriga Clarke, 1950 (Ethmia); Journal of the Washington Academy of Sciences 40: 163. Holotype ${ }^{*}$, USA, California, Bodega Bay, 2 May 1937, E. C. Johnston. USNM Type No. 59638, USNM Genitalia Slide No. 10859. [Elachistidae: Ethmiinae]

brocha Adamski \& Brown, 2001 (Glyphidocera); Proceedings of the Entomological Society of Washington 103: 970. Holotype $\sigma^{*}$, Venezuela, Territorio Federal Amazona, Cerro de la Neblina, base camp, $140 \mathrm{~m}, 0^{\circ} 50^{\prime} \mathrm{N}, 66^{\circ} 9^{\prime} \mathrm{W}, 13-20$ February 1984, D. Davis \& T. McCabe. USNM Genitalia Slide No. 81642. [Glyphidoceridae]

bruneri Busck, 1934 (Hypercallia); Entomologica Americana 13: 161. Holotype ${ }^{\star}$, Cuba, Baracoa, Ote., 21-30 April 1929, S. C. Bruner \& L. Boucle. Genitalia Slide AB Mar. 28, 1932. [Oecophoridae: Oecophorinae]

brunneipes Hodges, 1962 (Anoncia); Entomologica Americana 42: 99. Holotype ơ, USA, California, San Diego Co., San Diego, 20 June 1911, W. S. Wright. USNM Type No. 66085, USNM Genitalia Slide No. 4047. [Cosmopterigidae: Cosmopteriginae]

brunnella Adamski \& Brown, 2001 (Glyphidocera); Proceedings of the Entomological Society of Washington 103: 994. Holotype $\&$, Venezuela, Territorio Federal Amazona, Cerro de la Neblina, base camp, $140 \mathrm{~m}, 0^{\circ} 50^{\prime} \mathrm{N}, 66^{\circ} 9^{\prime} \mathrm{W}, 24$ November-1 December 1984, R. Brown. USNM Genitalia Slide No. 81637. [Glyphidoceridae]

brunneus Busck, 1903 (Prostomeus); Proceedings of the U.S. National Museum 25: 838. Syntype $₹$, USA, Florida, [no date], Beutenmüller collection. USNM Type No. 6367, J. F. G. Clarke Genitalia Slide No. 2950. [Gelechiidae: Gelechiinae]

busckella Ely, 1910 (Ptycerata); Proceedings of the Entomological Society of Washington 12: 69. Holotype ${ }^{\star}$, USA, Connecticut, East River, 28 July 1909, C. Ely. USNM Type No. 12881, USNM Genitalia Slide No. 10674. [Gelechiidae: Gelechiinae]

buscki Duckworth, 1964 (Lethata); Proceedings of the U.S. National Museum 116: 102. Holotype $\sigma^{*}$, British Honduras, Punta Gorda, July 1933, G. H. \& J. Sperry. USNM Type No. 66685, USNM Genitalia Slide No. 13381. [Elachistidae: Stenomatinae]

busckiella Engel, 1907 (Synallagma); Entomological News 18: 278. Syntype ․ USA, Pennsylvania, Pittsburgh, 3 July 1906, H. Engel. USNM Type No. 10286. Although the date on the specimen does not agree with the dates presented in the original description, we suspect that it is one of type series. Other syntypes are present in the Carnegie Museum. [Coleophoridae: Momphinae] 
busckiella Kearfott, 1903 (Gnorimoschema); Journal of the New York Entomological Society 11: 158. Syntypes (2ఠ), USA, New Jersey, Caldwell, r.f. gall on shoots of Aster patens, em. October. Described from 55 specimens, Kearfott clearly indicates "Type U. S. Nat. Mus."; there are two specimens labeled "Type." USNM Type No. 6818, USNM Genitalia Slide No. 6936. [Gelechiidae: Gelechiinae]

busiris Hodges, 1966 (Batrachedra); Transactions of the American Entomological Society 92: 606. Holotype ${ }^{\star}$, USA, Florida, Highlands Co., Parker Islands, 26-29 May 1964, R. W. Hodges. USNM Type No. 67856. [Batrachedridae]

cabima Busck, 1912 (Gonada); Smithsonian Miscellaneous Collections 59(4): 6. Holotype $\sigma^{\star}$, Panama, Cabima, May, A. Busck. USNM Type No. 14532, USNM Genitalia Slide No. 69438. [Oecophoridae: Oecophorinae]

cacula Hodges, 1999 (Chionodes); Moths of America north of Mexico, fasc. 7.6: 61. Holotype $\sigma^{\star}$, USA, Florida, Highlands Co., Archbold Biological Station, Lake Placid, 8-15 May 1964, R. W. Hodges. [Gelechiidae: Gelechiinae]

caia Hodges, 1986 (Dichomeris); Moths of America north of Mexico, fasc. 7.1: 62. Holotype $\sigma^{\star}$, USA, Illinois, Putnam Co., 11 June 1947, M. O. Glenn. USNM Genitalia Slide No. 9133. [Gelechiidae: Dichomeridinae]

cajonensis Clarke, 1941 (Agonopterix); Proceedings of the U.S. National Museum 90: 82. Holotype ${ }^{\star}$, USA, California, San Bernardino Co., Cajon Valley, 25 June 1937, G. \& J. L. Sperry. USNM Type No. 52948, USNM Genitalia Slide No. 154. [Elachistidae: Depressariinae]

calator Hodges, 1966 (Batrachedra); Transactions of the American Entomological Society 92: 607. Holotype ${ }^{*}$, Mexico, Hildago, Real del Monte, 12 April 1909, Van Ostrand. USNM Type No. 67857, USNM Genitalia Slide No. 5072. [Batrachedridae]

californica Busck, 1907 (Paralechia); Proceedings of the Entomological Society of Washington 8: 92. Holotype + , USA, California, Los Angeles Co. [no date]. USNM Type No. 9896, USNM Genitalia Slide No. 6578. [Gelechiidae: Gelechiinae]

caliginosella Busck, 1904 (Ethmia); Journal of the New York Entomological Society 12: 44. Holotype 9 , USA, Colorado, Silverton, 12,000', 1903, C. P. Gillette. USNM Type No. 7733. [Elachistidae: Ethmiinae] 
callosella Barnes \& Busck, 1920 (Agonopteryx); Contributions to the Natural History of the Lepidoptera of North America 4: 231. Lectotype 9 , USA, California, San Bernardino, 1-7 June. USNM Type No. 22665, USNM Genitalia Slide No. 1364. Designated by Hodges (1974). [Elachistidae: Depressariinae]

calusella Kaila, 1996 (Elachista); Entomologica Scandinavica 27: 224. Holotype $\sigma^{\star}$, USA, Florida, Fisheating Creek, Palmdale, 7-10 May 1964, R. W. Hodges. USNM Genitalia Slide No. 89276. [Elachistidae: Elachistinae]

cambiella Busck, 1915 (Psacaphora); Proceedings of the Entomological Society of Washington 17: 81. Holotype + , USA, Montana, Evans, in cambium of Salix, 15 May 1913, J. Brunner. USNM Type No. 19224, USNM Genitalia Slide No. 4993. [Elachistidae: Agonoxeniinae]

canadensis Busck, 1902 (Depressaria); Proceedings of the U.S. National Museum 24: 744. Holotype 9 , Canada, Manitoba, Winnipeg, [no date], A. W. Hanham. USNM Type No. 6127, USNM Genitalia Slide No. 176. [Elachistidae: Depressariinae]

canariella Busck, 1908 (Cryptolechia); Proceedings of the U.S. National Museum 35: 195. Syntypes $\left(10^{\star}, 2\right.$ $)$, USA, Arizona, Huachuca Mountains [no date]. USNM Type No. 11940, USMN Genitalia Slides Nos. 843, 844, 845. One of the females lacks a "Type" label. [Oecophoridae: Oecophorinae]

canella Busck, 1904 (Depressaria); Proceedings of the U.S. National Museum 27: 764. Holotype $\sigma^{*}$, USA, Washington, Pullman, 2 September, C. V. Piper. USNM Type No. 7817, USNM Genitalia Slide No. 401, USNM Wing Slide No. 409. [Elachistidae: Depressariinae]

canicoma Clarke, 1986 (Asymphorodes); Smithsonian Contributions to Zoology 416: 241. Holotype $o^{\star}$, Marquesas Archipelago, Fatu Hiva, Mt. Upe, 2025', 3 April 1968, J. F. G. \& T. M. Clarke. USNM Type No. 100801, USNM Genitalia Slide No. 24924. [Cosmopterigidae: Cosmopteriginae]

canitiana Wu \& Park, 1998 (Hygroplasta); Insecta Koreana 15: 8. Holotype ơ, Sri Lanka, Kan. District, Laksapana, 1200', 23-29 September 1970, O. S. Flint, Jr. USNM Genitalia Slide No. 89216. [Lecithoceridae]

cannescens Clarke, 1956 (Pseuderotis); Entomological News 17: 256. Holotype + , Argentina, Tigre, [no date], F. Bourquin. USNM Type No. 63272, USNM Genitalia Slide No. 86871. [Peleopodidae] 
canofusella Clarke, 1947 (Chionodes); Journal of the Washington Academy of Sciences 37: 248. Holotype ${ }^{*}$, USA, Texas, Brooks Co., Encantada, 28 November 1940, Hunt \& McGarr. USNM Type No. 58239, USNM Genitalia Slide No. 7880. [Gelechiidae: Gelechiinae]

canor Hodges, 1999 (Chionodes); Moths of America north of Mexico, fasc. 7.6: 221. Holotype $\diamond^{\star}$, USA, Arizona, Coconino Co., Fort Valley, $7.5 \mathrm{mi}$ NW Flagstaff, 8 August 1961, R. W. Hodges. [Gelechiidae: Gelechiinae]

canusella Chamber (Harpalyce), Canadian Entomologist 6: 235. Pseudotype (uncertain sex), USA, Texas [no date]. [Elachistidae: Stenomatinae]

capella Busck, 1940 (Mompha); Bulletin of the Southern California Academy of Sciences 39: 88. Holotype $\approx$, USA, New York, Massapequa, Long Island, r.f. seed capsules of Crocanthemum sp., em. 8 July 1937, L. J. Bottimer. [Coleophoridae: Momphinae]

carabophanes Meyrick, 1932 (Antaeotricha); Exotic Microlepidoptera 4: 289. Holotype ^, Colombia, San Antonio, November, Fassel. USNM Genitalia Slide No. 14060. [Elachistidae: Stenomatinae]

carbonifer Busck, 1914 (Cerconota); Proceedings of the U.S. National Museum 47: 48. Holotype $\sigma^{\star}$, Panama, Porto Bello, March 1912, A. Busck. USNM Type No. 16728, USNM Genitalia Slide No. 13643. [Elachistidae: Stenomatinae]

caritella Busck, 1908 (Gerdana); Proceedings of the U.S. National Museum 35: 193. Syntypes $\left(1{ }^{*}, 4 \circ, 1\right.$ uncertain sex), USA, Maryland, Montgomery Co., Plummers Island, July 1903, A. Busck. J. F. G. Clarke Genitalia Slide Nos. 264 ( $\left.{ }^{\circ}\right)$ and $959\left({ }^{\ngtr}\right)$, USNM Genitalia Slide No. 87284. One specimen with abdomen lacking. [Autostichidae: Symmocinae]

carnicheella Jäckh, 1978 (Scythris glacialis); Bolletino del Museo Civico di Storia Naturali 5: 11. Holotype $\sigma^{*}$, Italy, Alpe Carniche, Sappada, Passo Houbolt, 2300 m, 22 July 1935, L. Rocca. USNM Genitalia Slide No. 87772. According to the original description, deposited in Museo Civico Storia Naturale, Genova, Italy. [Xyloryctidae: Scythridinae]

carribea Busck, 1910 (Glyphidocera); Bulletin of the Department of Agriculture, Trinidad 9 (66): 244. Holotype $\sigma^{\star}$, British West Indies, Trinidad, June 1905, A. Busck. USNM Type No. 13383, USNM Genitalia Slide 82504. [Glyphidoceridae]

caryae Clarke, 1941 (Psilocorsis); Proceedings of the U.S. National Museum 90: 215. Holotype o* USA, Texas, Cuero, 13 September 1910, M. M. High. USNM Type No. 52866. [Amphisbatidae] 
caryaevorella Packard, 1886 (Gelechia); Annual Report of the Department of Agriculture, not found. [Gelechiidae: Gelechiinae]

castanella Adamski \& Brown, 2001 (Glyphidocera); Proceedings of the Entomological Society of Washington 103: 997. Holotype $\$$, Venezuela, Territorio Federal Amazona, Cerro de la Neblina, base camp, $140 \mathrm{~m}, 0^{\circ} 50^{\prime} \mathrm{N}, 66^{\circ} 9^{\prime} \mathrm{W}, 4-12$ February 1984, D. Davis \& T. McCabe. USNM Genitalia Slide No. 81638. [Glyphidoceridae]

catalinella Busck, 1907 (Gelechia); Journal of the New York Entomological Society 15: 136. Holotype + , USA, Arizona, Catalina Springs, August. USNM Type No. 10319, USNM Genitalia Slide No. 10685. [Gelechiidae: Gelechiinae]

caudatae Clarke, 1934 (Gelechia); Canadian Entomologist 65: 175. Holotype o*, USA, Washington, Pullman, r.f. Salix caudata, 25 July 1933, J. F.G. Clarke. USNM Genitalia Slide No. 6752. [Gelechiidae: Gelechiinae]

caulisivena Wu \& Park, 1999 (Timyra); Korean Journal of Systematic Zoology 15: 206. Holotype $\sigma^{*}$ Sri Lanka, Gal. District, Kanneliya, 200', 15-17 October 1976, Hevel, Dietz, Karunaratne \& Balasocriya. USNM Genitalia Slide No. 88920. [Lecithoceridae]

cautor Hodges, 1999 (Chionodes); Moths of America north of Mexico, fasc. 7.6: 142. Holotype ${ }^{*}$, USA, Texas, Brewster Co., Big Bend National Park, Green Gulch, 30 November 1985, E. C. Knudson. R. W. Hodges Genitalia Slide No. 5111. [Gelechiidae: Gelechiinae]

ceanothiella Busck, 1904 (Gelechia); Proceedings of the U.S. National Museum 27: 760. Lectotype $\sigma^{*}$, Canada, British Columbia, Kaslo, 21 June 1903, r.f. Ceanothus sanguineus, H. Dyar. USNM Type No. 7863, USNM Genitalia Slide No. 15142. Designated by Hodges (1999). [Gelechiidae: Gelechiinae]

cephalonthiella Chambers, 1871 (Laverna); Canadian Entomologist 3: 221. Syntypes (o*, \%), USA, Kentucky, [no date], r.f. mines on leaves of Cephalanthus occidentalis. [Coleophoridae: Momphinae]

ceraena Wu \& Park, 1999 (Doxogenes); Korean Journal of Systematic Zoology 15: 217. Holotype $\sigma^{\star}$, Sri Lanka, Col. District, Tunmodera, 200', 17 November 1970, O. S. Flint, Jr. USNM Genitalia Slide No. 89157. [Lecithoceridae] 
cerasella Kaila, 1996 (Elachista); Entomologica Scandinavica 27: 235. Holotype o*, USA, Nebraska, Cherry Co., Fort Niobrara, 19 June 1983, R. W. Hodges. USNM Genitalia Slide No. 89277. [Elachistidae: Elachistinae]

cerussata Walsingham, 1911 (Gelechia); Biologia Centrali-Americana, Lepidoptera Heterocera 4: 61. Holotype $\sigma^{*}$, Mexico, Veracruz, Cordoba, May 1908, F. Knab, Walsingham No. 5977. Abdomen lacking. [Gelechiidae: Gelechiinae]

ceryx Hodges, 1999 (Chionodes); Moths of America north of Mexico, fasc. 7.6: 172. Holotype $\diamond^{\star}$, USA, Florida, Monroe Co., N. Key Largo, 20 November 1976, D. C. Ferguson. [Gelechiidae: Gelechiinae]

cestrivora Clarke, 1950 (Gnorimoschema); Journal of the Washington Academy of Sciences 40: 288. Holotype ${ }^{*}$, Argentina, Tucumán, [no date], r.f. galls on Cestrum lorentzanum, K. J. Hayward. USNM Type No. 59424, USNM Genitalia Slide No. 10672. [Gelechiidae: Gelechiinae]

ceylonica Park, 2001 (Frisilia); in Park \& Wu, Insecta Koreana 18: 144. Holotype o*, Sri Lanka, Anu. District, Wildlife Soc. Bungalo, Hunuwilagama, 200', Wilpatu, 10-19 March 1970, D. Davis \& B. Rowe. USNM Genitalia Slide No. 87833. [Lecithoceridae]

chaetosoma Clarke, 1962 (Agonopterix); Entomological News 73: 93. Holotype o*, Japan, Honsyû, Kii, Nati, 21 June 1957, larva in cylinder of tied leaf of Fagara schinifolia, T. Kodama. USNM Type No. 65829, USNM Genitalia Slide No. 25939. [Elachistidae: Depressariinae]

chalcopterus Clarke, 1986 (Asymphorodes); Smithsonian Contributions to Zoology 416: 217. Holotype ${ }^{*}$, Marquesas Archipelago, Hiva Oa, Mt. Feani, 3400', 1 March 1968, J. F. G. \& T. M. Clarke. USNM Type No. 100778, USNM Genitalia Slide No. 25170. [Cosmopterigidae: Cosmopteriginae]

chalcosoma Clarke, 1986 (Asymphorodes); Smithsonian Contributions to Zoology 416: 254. Holotype $\sigma^{*}$, Marquesas Archipelago, Nuku Hiva, Tunoa Ridge, 2900', 23 January 1968. USNM Type No. 100810, USNM Genitalia Slide No. 25108. [Cosmopterigidae: Cosmopteriginae]

chambersella Dyar, 1903 (Ethmia); Journal of the New York Entomological Society 10 (1902): 208. Holotype + , USA (no other data). USNM Type No. 6625, USNM Genitalia Slide No. 86866. Preoccupied; replaced by Durrantia obiterella Busck, 1908, Proceedings of the U.S. National Museum 35: 207. [Peleopodidae] 
chaonella Park, 1995 (Anarsia); Tropical Lepidoptera 6: 61. Holotype $\sigma^{*}$, Taiwan, Tapei Co., Taihoku, 6 August 1946, S. Issiki. USNM Genitalia Slide No. 87398. [Gelechiidae: Gelechiinae]

charagma Clarke, 1971 (Gonionota); Smithsonian Contributions to Zoology 95: 9. Holotype $\sigma^{\star}$, Brazil, Nova Teutonia, April 1948, F. Plaumann. USNM Type No. 71087, USNM Genitalia Slide No. 68744. [Oecophoridae: Oecophorinae]

chenopodiella Busck, 1916 (Gnorimoschema); Proceedings of the Entomological Society of Washington 18: 148. Holotype i, USA, Massachusetts, W Springfield, r.f. pigweed, 7 September 1915, H. E. Smith. USNM Type No. 20203. Abdomen lacking. [Gelechiidae: Gelechiinae]

chersopsamma Meyrick, 1932 (Anapatris); Exotic Microlepidoptera 4: 287. Holotype $q$, Panama, Canal Zone, Corazal, March 1911, A. Busck. [Elachistidae: Stenomatinae]

chilibrella Busck, 1914 (Teratomorpha); Proceedings of the U.S. National Museum 47: 31. Holotype $\sigma^{*}$, Panama, Alhajuela, April 1911, A. Busck. USNM Type No. 16754, USNM Genitalia Slide No. 4086. [Oecophoridae: Oecophorinae]

chinanensis Park, 2003 (Issikiopteryx); Journal of Asia-Pacific Entomology 6: 16. Holotype $\sigma^{*}$, Taiwan, Kaohsiung Co., Keinansan [=Chiananshan], 14 August 1933, S. Issiki. Specimen not found. [Lecithoceridae]

chionostigma Walsingham, 1911 (Telphusa); Biologia Centrali-Americana, Lepidoptera Heterocera 4: 57. Holotype + , Panama, Canal Zone, Tabernilla, [no date], A. Busck, Walsingham No. 5831. Abdomen lacking. [Gelechiidae: Gelechiinae]

chiquitella Busck, 1910 (Gnorimoschema); Proceedings of the Entomological Society of Washington 11: 176. Holotype 9 , USA, New Mexico, Mesilla, [no date], C. N. Ainsley. USNM Type No. 12683. Abdomen lacking. [Gelechiidae: Gelechiinae]

chisosensis Hodges, 1978 (Cosmopterix); Moths of America north of Mexico, fasc. 6.1: 31. Holotype o*, USA, Texas, Brewster Co., Big Bend National Park, Chisos Mountains, Green Gulch, 5500', 6 June 1973, R. W. Hodges. USNM Genitalia Slide No. 3890. [Cosmopterigidae: Cosmopteriginae]

chlorina Kearfott, 1911 (Stenoma); Entomological News 22: 126. Syntypes (2๔\% 3), Brazil, São Paulo [no date]. USNM Type No. 16714, USNM Genitalia Slide Nos. 13312, 13313, 13314, 13316, 13319. Labial palpus and left mesothoracic leg in gelatin capsule; one pinned specimen missing. [Elachistidae: Stenomatinae] 

Holotype $\sigma^{*}$, Panama, La Chorrera, 1912, A. Busck. USNM Type No. 16751, USNM Genitalia Slide No. 4081. [Amphisbatidae]

chrautis Hodges, 1974 (Agonopterix); Moths of America north of Mexico, fasc. 6.2: 46. Holotype 9 , USA, New Mexico, Jemez Springs, 24-31 July. USNM Genitalia Slide No. 137. [Elachistidae: Depressariinae]

chrysophanes Clarke, 1986 (Asymphorodes); Smithsonian Contributions to Zoology 416: 223. Holotype ${ }^{\star}$, Marquesas Archipelago, Hiva Oa, Atuona, 12 February 1968, J. F. G. \& T. M. Clarke. USNM Type No. 100789, USNM Genitalia Slide No. 25007. [Cosmopterigidae: Cosmopteriginae]

chungchinmookara Adamski \& Brown, 2001 (Glyphidocera); Proceedings of the Entomological Society of Washington 103: 981. Holotype $\sigma^{*}$, Venezuela, Territorio Federal Amazona, Cerro de la Neblina, camp VII, 051'N, 6558'W, 1850 m, 2-4 December 1984, R. Brown. USNM Genitalia Slide No. 81457. [Glyphidoceridae]

cibus Hodges, 1999 (Chionodes); Moths of America north of Mexico, fasc. 7.6: 98. Holotype $\sigma^{*}$, USA, Texas, Cameron Co., Laguna Atascosa, 22 November 1973, A. \& M. E. Blanchard. USNM Genitalia Slide No. 14764. [Gelechiidae: Gelechiinae]

cicatricula Clarke, 1986 (Asymphorodes); Smithsonian Contributions to Zoology 416:

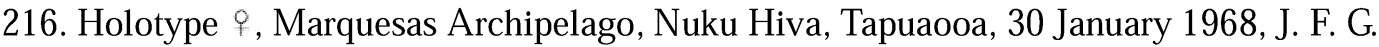
\& T. M. Clarke. USNM Type No. 100780, USNM Genitalia Slide No. 24943. [Cosmopterigidae: Cosmopteriginae]

ciliella Busck, 1908 (Cryptolechia); Proceedings of the U.S. National Museum 35: 196. Holotype $\diamond^{*}$, USA, Arizona, Pima Co., Baboquavaria (=Baboquivari) Mountains, 15-30 July 1903, O. C. Poling. USNM Type No. 11942, USNM Genitalia Slide No. 891. [Oecophoridae: Oecophorinae]

cinnamomea Clarke, 1978 (Alynda); Smithsonian Contributions to Zoology 273: 56. Holotype $\sigma^{*}$, Chile, Llanquihue, Petrohue, 10 March 1959, J. F. G. Clarke. USNM Type No. 73721, USNM Genitalia Slide No. 24285. Right fore- and hindwing lacking. [Oecophoridae: Oecophorinae]

circitor Hodges, 1969 (Perimede); Smithsonian Contributions to Zoology 18: 13. Holotype + , USA, Arkansas, Washington Co., Devil's Den State Park, 18 July 1966, R. W. Hodges. USNM Type No. 69790, USNM Genitalia Slide No. 4811. [Cosmopterigidae: Chrysopeleiinae] 
citrantha Diakonoff, 1968 (Tonica); Bulletin of the U.S. National Museum 257 (1967): 184. Holotype 9 , Philippine Islands, Luzon, Los Baños, [no date], Woodward. USNM Genitalia Slide No. 89967. [Oecophoridae: Oecophorinae]

citricolella Chambers, 1880 (Blastobasis); in Comstock, Report of the Commissioner of Agriculture, 1879: 206. Lectotype ${ }^{\circ}$, USA, Florida, Jacksonville, r.f. dry orange, em. 17 March 1880. USNM Type No. 3774. Right forewing, left hindwing, and abdomen lacking. Designated by Adamski \& Hodges (1996). [Coleophoridae: Blastobasinae]

citrifoliella Chambers, 1880 (Nothris); Journal of the Cincinnati Society of Natural History 2: 184. Lectotype $\sigma^{*}$, USA, Florida, October 1879. Abdomen lacking. Designated by Hodges (1986). [Gelechiidae: Dichomeridinae]

citrinella Barnes \& Busck, 1920 (Epithectis); Contributions to the Natural History of the Lepidoptera of North America 4: 224. Holotype $\diamond^{*}$, USA, Arizona, Palmerlee [no date]. USNM Genitalia Slide No. 6598. [family placement uncertain]

citrinodema Wu \& Park, 1999 (Timyra); Korean Journal of Systematic Zoology 15: 210. Holotype ${ }^{*}$, Sri Lanka, Gal. District, Udugama, Kanneliya Jungle, 400', 6-12 October 1973, at black light, Krombien, Karunaratna, Fernando \& Ferdinando. USNM Genitalia Slide No. 88807. [Lecithoceridae]

clandestinella Busck, 1906 (Cosmopteryx); Proceedings of the U.S. National Museum 30: 712. Holotype ${ }^{\star}$, USA, Twining City, District of Columbia, r.f. Tanicum, em. 1899-1902. USNM Type No. 9777. Abdomen lacking. [Cosmopterigidae: Cosmopteriginae]

claripennis Busck, 1914 (Stenoma); Proceedings of the U.S. National Museum 47: 39. Holotype i, Panama, Taboga Island, June 1911, A. Busck. USNM Type No. 16710, USNM Genitalia Slide No. 13751. [Elachistidae: Stenomatinae]

clarissa Busck, 1914 (Ethmia); Insecutor Inscitiae Menstruus 2: 56. Holotype o*, Cuba, [no date], W. Schaus. USNM Type No. 18269, USNM Genitalia Slide No. 87687. [Elachistidae: Ethmiinae]

clarkei Hodges, 1999 (Chionodes); Moths of America north of Mexico, fasc. 7.6: 228. Holotype $\odot^{\star}$, USA, Oregon, Harney Co., Fish Lake, Steens Mountain, 7100', 12 July 1962, J. F. G. Clarke. [Gelechiidae: Gelechiinae]

clarkei Kaila, 1995 (Perittia); SHILAP Revista de Lepidopterologia 23: 266. Holotype ${ }^{\star}$, Washington, Wawawai, 18 April 1929, J. F. G. Clarke. USNM Genitalia Slide No. 87317, USNM Wing Slide No. 87318. [Elachistidae: Elachistinae] 
clarkei Keifer, 1936 (Agonopteryx); Bulletin of the Southern California Academy of Sciences 35: 10. Holotype + , USA, California, El Dorado Co., Missouri Flat, 28 May 1935, r.f. Artemisia vulgaris, em. 27 June 1935. USNM Genitalia Slide No. 58. [Elachistidae: Depressariinae]

clarkei Powell, 1973 (Ethmia); Smithsonian Contributions to Zoology 120: 117. Holotype ox, Mexico, Quintana Roo, Isla de Mujeres, near Puerto Juarez, 29 March 1960, J. F. G. Clarke, Bredin Expedition. USNM Genitalia Slide No. 98453. Right forewing lacking. [Elachistidae: Ethmiinae]

claudiella Kearfott, 1907 (Mompha); Canadian Entomologist 39: 212. Syntypes (1 $\sigma^{*}, 1 \%$ ), Canada, Manitoba, Rounthwaite, July, C. Marmont. USNM Type No. 9871, USNM Genitalia Slide No. $4954\left({ }^{\star}\right)$. One syntype not found. [Coleophoridae: Momphinae]

clavata Park, 2001 (Alciphanes); in Park \& Wu, Insecta Koreana 18: 142. Holotype $\odot^{\star}$, Sri Lanka, Jap. District, Chundikkulan Sanctuary, 25', 7 November 1976, G. Hevel \& D. Davis. [Lecithoceridae]

clevelandi Busck, 1914 (Borkhausenia); Proceedings of the U.S. National Museum 47: 32. Holotype $o^{\star}$, Panama, La Chorrera, May 1912, A. Busck. USNM Type No. 16693, USNM Genitalia Slide No. 69568. [Oecophoridae: Oecophorinae]

cocae Busck, 1931 (Eucleodora); Proceedings of the Entomological Society of Washington 33: 60. Holotype 9 , Peru, Otuzco, September 1930, Mille. USNM Type No. 43310, USNM Genitalia Slide No. 15642. [Oecophoridae: Oecophorinae]

cockerella Busck, 1903 (Aristotelia); Proceedings of the U.S. National Museum 25: 800. Holotype 9 , USA, New Mexico, Mesilla Park, [no date], T. D. A. Cockerell. USNM Type No. 6351, USNM Genitalia Slide No. 10878. [Gelechiidae: Gelechiinae]

cockerelli Busck, 1903 (Gelechia); Proceedings of the U.S. National Museum 25: 871. Holotype , USA, New Mexico, Mesilla Park, 13 May, T. D. A. Cockerell. USNM Type No. 6384, USNM Genitalia Slide No. 7607. [Gelechiidae: Gelechiinae]

coenophyes Diakonoff, 1968 (Metathrinca); Bulletin of the U.S. National Museum 257 (1967): 119. Holotype $\sigma^{*}$, Philippine Islands, Luzon, Mt. Apo 70B, 17 March 1945, J. G. Franclemont. USNM Genitalia Slide No. 89938. [Xyloryctidae: Xyloryctinae]

coffeaella Busck, 1925 (Auximobasis); Communicições, Estudo e Dabellacão de Praga Caféeira, São Paulo, Pub. 13: 13. Holotype ${ }^{*}$, Brazil, São Paulo, r.f. coffee, A. Neiva. USNM Type No. 28167. [Coleophoridae: Blastobasinae] 
cognata Wu \& Park, 1998 (Deltoplastis); Insecta Koreana 15: 6. Holotype o , Sri Lanka, Kan. District, Kandy, 2100', Udawattakele Sanctuary, 10-23 January 1970, D. Davis \& B. Rowe. USNM Genitalia Slide No. 89000. [Lecithoceridae]

cologramma Clarke, 1971 (Gonionota); Smithsonian Contributions to Zoology 95: 5. Holotype $\sigma^{*}$, Venezuela, Aragua, Rancho Grande, 1100 m, 10-21 February 1969, W. D. Duckworth \& R. Dietz. USNM Type No. 71085, USNM Genitalia Slide No. 68757. [Oecophoridae: Oecophorinae]

coloradensis Busck, 1903 (Gelechia); Proceedings of the U.S. National Museum 25: 857. Holotype + , USA, Colorado, Glenwood Springs [no date]. USNM Type No. 6374, USNM Genitalia Slide No. 9911. [Gelechiidae: Gelechiinae]

colubrinae Busck, 1903 (Recurvaria); Proceedings of the U.S. National Museum 25: 810. Holotype (uncertain sex), USA, Texas, Rockport, r.f. Psylla galls on Colubrina texensis, August 1894, E. A. Schwarz. USNM Type No. 6354. Specimen not found. [Gelechiidae: Gelechiinae]

comma Busck, 1911 (Stenoma); Proceedings of the U.S. National Museum 40: 218. Syntypes $\left(2{ }^{\star}\right)$, French Guiana, Cayenne, [no date], W. Schaus. USNM Type No. 13578, USNM Genitalia Slide No. 15554. [Elachistidae: Stenomatinae]

comosae Hodges, 1966 (Batrachedra); Transactions of the American Entomological Society 92: 626. Holotype $\diamond^{\star}$, Puerto Rico, near Corozal, 25 July 1951, r.f. Ananas comosus, O. D. Courtney \& J. D. Crump. USNM Type No. 67873, USNM Genitalia Slide No. 5137. [Batrachedridae]

complicata Clarke, 1951 (Coptotelia); Acta Zoologica Lilloana 11: 340. Holotype ơ, Brazil, Santa Catarina, 1936, F. Plaumann. USNM Type No. 61114. [Oecophoridae: Oecophorinae]

conchita Busck, 1920 (Gonioterma); Insecutor Inscitiae Menstruus 8:93. Holotype o*, Guatemala, Cayuga, [no date], W. Schaus. USNM Type No. 22315, W. D. Duckworth Genitalia Slide No. 2575. [Elachistidae: Stenomatinae]

concinusella Chambers, 1875 (Gelechia); Cincinnati Quarterly Journal of Science 2: 253. Syntype 9 , USA, Texas [no date]. USNM Type No. 448, J. F. G. Clarke Genitalia Slide No. 3433. [Gelechiidae: Gelechiinae] 
concolor Adamski \& Maier, 2003 (Holcocera); Proceedings of the Entomological Society 510 of Washington 105: 145. Holotype $\sigma^{\star}$, USA, Massachusetts, Barnstable, 25 May 1951, C. P. Kimball. USNM Genitalia Slide No. 82257. [Coleophoridae: Blastobasinae]

concolorella Beutenmüller, 1888 (Cryptolechia); Entomologica Americana 4: 30. Holotype $\sigma^{*}$, USA, Nevada [no date]. USNM Type No. 431, USNM Genitalia Slide No. 858. [Oecophoridae: Oecophorinae]

concolorella Chambers, 1875 (Eriphia); Canadian Entomologist 7: 55. Holotype + , USA, Texas [no date]. Specimen not found. [Cosmopterigidae: Chrysopeleiinae]

concolorella Chambers, 1875 (Elachista); Canadian Entomologist 7: 55. Pseudotype (uncertain sex), USA, Texas [no date]. [Cosmopterigidae: Chrysopeleiinae]

condaliavorella Busck, 1900 (Trichotaphe); Proceedings of the U.S. National Museum 23: 232. Lectotype $\sigma^{*}$, USA, Florida, Palm Beach, r.f. Condalia ferrea, [no date], H. Dyar. USNM Type No. 4940, USNM Genitalia Slide No. 10643. Designated by Hodges (1986). [Gelechiidae: Dichomeridinae]

confamulella Heinrich, 1921 (Holcocera); Journal of Agricultural Research 20: 818. Holotype $\sigma^{*}$, USA, Texas, Brownsville, Smith Point, em. 10 April 1919, F. H. Barber. USNM Type No. 23742, Genitalia and Wing Slide CH-26, July 1920. [Coleophoridae: Blastobasinae]

confluentella Dietz, 1910 (Holcocera); Transactions of the American Entomological Society 36: 36. Lectotype + , USA, Massachusetts, Cohasset, 5 July 1907, O. Bryant. USNM Genitalia Slide No. 80999. Designated by Adamski \& Hodges (1996). [Coleophoridae: Blastobasinae]

coniophora Braun, 1948 (Elachista); Memoirs of the American Entomological Society 13: 51. Holotype ${ }^{\star}$, USA, California, San Diego, April, Ricksecker. USNM Type No. 34827, USNM Genitalia Slide No. 89273. [Elachistidae: Elachistinae]

constancei Clarke, 1947 (Depressaria); Journal of the Washington Academy of Sciences 37: 5. Holotype ${ }^{\star}$, USA, California, Siskiyou Co., Yreka, 24 May 1946, r.f. Lomatium californicum (Nutt.), J. F. G. C. Clarke. USNM Type No. 58007. [Elachistidae: Depressariinae]

contrasta Clarke, 1964 (Gonionota); Proceedings of the U.S. National Museum 115: 64. Holotype $\sigma^{\star}$, Peru, Tingo Maria, 25 November 1949, H. A. Allard. USNM Type No. 66373, USNM Genitalia Slide No. 68952. [Oecophoridae: Oecophorinae] 
convoluta Duckworth, 1970 (Peleopoda); Smithsonian Contributions to Zoology 48: 8.

Holotype * $^{*}$ Venezuela, Aragua, Rancho Grande, 1100 m, 7 August 1943, R. Lichy. USNM Type No. 70838, USNM Genitalia Slide No. 87127. Right fore- and hindwing lacking. [Peleopodidae]

copa Hodges, 1986 (Dichomeris); Moths of America north of Mexico, fasc. 7.1: 92. Holotype $\sigma^{\star}$, USA, New York, Ithaca, Snyder Heights, 1100', 16 July 1975, J. G. Franclemont. [Gelechiidae: Dichomeridinae]

copia Clarke, 1957 (Batrachedra); Acta Zoologica Mexicana 2: 3. Holotype + , Mexico, Tlalnepantla, r.f. Agave sp., 13 February 1956, I. B. Barrera. USNM Type No. 63452, USNM Genitalia Slide No. 5100. [Batrachedridae]

coquillettella Busck, 1902 (Gnorimoschema); Proceedings of the U.S. National Museum 25: 405. Holotype ơ, USA, California, Los Angeles, [date illegible], D. W. Coquillette. USNM Genitalia Slide No. 6956. [Gelechiidae: Gelechiinae]

coquillettella Busck, 1907 (Ethmia); Proceedings of the Entomological Society of Washington 8: 95. Holotype $\sigma^{*}$, USA, California, [no date], D. W. Coquillett. USNM Type No. 9900. [Elachistidae: Ethmiinae]

cora Busck, 1914 (Gonioterma); Proceedings of the U.S. National Museum 47: 53. Holotype $\sigma^{*}$, Panama, Trinidad River, March 1912, A. Busck. USNM Type No. 16742, USNM Genitalia Slide No. 13013. [Elachistidae: Stenomatinae]

coranella Dyar, 1902 (Ethmia); Journal of the New York Entomological Society 10: 207. Lectotype ${ }^{\circ}$, USA, Texas, Kerrville, [no date], W. Barnes. USNM Type No. 6624. Designated by Powell (1973). [Elachistidae: Ethmiinae]

cornuta Busck, 1914 (Dichomeris); Proceedings of the U.S. National Museum 47: 20. Holotype ơ Panama, Trinidad River, June 1912, A. Busck. USNM Type No. 16677. [Gelechiidae: Dichomeridinae]

costalis Busck, 1914 (Dichomeris); Proceedings of the U.S. National Museum 47: 18. Syntypes $\left(1 \sigma^{\circ}, 1^{\circ}\right)$, Panama, Tabogilla Island and Porto Bello, April and February 1912, A. Busck. USNM Type No. 16673. [Gelechiidae: Dichomeridinae]

costimacula Clarke, 1941 (Agonopterix); Proceedings of the U.S. National Museum 90: 102. Holotype ${ }^{*}$, USA, Maryland, Montgomery Co., Plummers Island, Potomac River, 3 March 1908, A. Busck. USNM Type No. 52080, USNM Genitalia Slide No. 346. [Elachistidae: Depressariinae] 
coticola Busck, 1913 (Gelechia); Journal of Entomology and Zoology (Pomona College) 510 5: 97. Holotype o , USA, California, San Diego Co., La Puerta, July, W. S. Wright \& G. Field. USNM Type No. 15609, USNM Genitalia Slide No. 6116. [Gelechiidae: Gelechiinae]

coverdalella Kearfott, 1903 (Anacampsis); Journal of the New York Entomological Society 11: 162. Holotype ${ }^{*}$, USA, Louisiana, Natchitoches Parish, [no date], G. Coverdale. USNM Type No. 6967, Genitalia Slide AB Nov. 10, 1935. The original description does not give the number of specimens examined, but it clearly states that the type is in USNM. [Gelechiidae: Gelechiinae]

cracens Hodges, 1978 (Sorhagenia); Moths of America north of Mexico, fasc. 6.1: 133. Holotype $\sigma^{*}$, USA, Idaho, Spirit Lake, 16-23 July. USNM Genitalia Slide No. 1368. [Cosmopterigidae: Chrysopeleiinae]

crambina Busck, 1920 (Stenoma); Insecutor Inscitiae Menstruus 8: 89. Holotype ơ, Mexico, Sierra de Guerrero [no date]. USNM Type No. 22320, USNM Genitalia Slide No. 13759. [Elachistidae: Stenomatinae]

crassicornella Dietz, 1910 (Holcocera); Transactions of the American Entomological Society 36: 32. Holotype ${ }^{*}$, USA, Florida [no date]. Abdomen lacking; other body parts in gelatin capsule. [Coleophoridae: Blastobasinae]

crataegella Busck, 1903 (Recurvaria); Proceedings of the U.S. National Museum 25: 811. Holotype 9 , USA, Pennsylvania, Hazleton, r.f. Crataegus tomentosus, 22 June 1899. USNM Type No. 6355, USNM Genitalia Slide No. 6263. [Gelechiidae: Gelechiinae]

cratia Hodges, 1974 (Agonopterix); Moths of North America north of Mexico, fasc. 6.2: 35. Holotype 9 , USA, Arizona, Coconino Co., Walnut Canyon, 6 1/3 mi ESE Flagstaff, 6500', 1 August 1964, J. G. Franclemont. [Elachistidae: Depressariinae]

crepida Hodges, 1986 (Dichomeris); Moths of America north of Mexico, fasc. 7.1: 118. Holotype ${ }^{*}$, USA, South Carolina, McClellanville, 20 March 1974, R. B. Dominick. [Gelechiidae: Dichomeridinae]

crescentifasciella Chambers, 1874 (Gelechia); Canadian Entomologist 5: 237. Holotype $\uparrow$, USA, Texas [no date]. USNM Type No. 446. Abdomen lacking. [Gelechiidae: Gelechiinae] 
crotalariella Busck, 1910 (Lipatia); Bulletin of the Department of Agriculture, Trinidad 9: 244. Holotype 9 , British West Indies, Trinidad, r.f. Crotalaria, em. 21 May 1910, F. W.

Urich. USNM Type No. 13382. Abdomen lacking. [Gelechiidae: Gelechiinae]

crotolariella Busck, 1900 (Aproaerema); Proceedings of the U.S. National Museum 23: 226. Holotype 9 , USA, Florida, Palm Beach, r.f. Crotolaria (= Crotalaria) pumilla, em. 7 March 1900, H. Dyar. USNM Type No. 4933, USNM Genitalia Slide No. 10678. Species name based on misspelling of plant genus. [Gelechiidae: Gelechiinae]

crotonella Bottimer, 1926 (Chaetocampa); Journal of Agricultural Research 33: 804. Holotype ${ }^{*}$, USA, Texas, Liberty, r.f. seeds of Croton glandulosus, 27 May 1924. USNM Type No. 27333. [Elachistidae: Agonoxeniinae]

crucifera Busck, 1914 (Psilocorsis); Proceedings of the U.S. National Museum 47: 25. Holotype ${ }^{\star}$, Panama, Trinidad River, June, A. Busck. USNM Type No. 16686. Abdomen lacking. [Amphisbatidae]

cryptopalpella Adamski, 1999 (Hypatopa); Proceedings of the Entomological Society of Washington 101: 439. Holotype ${ }^{*}$, West Indies, Dominica, 2 mi NW Pont Cass, May 1965, D. R. Davis. [Coleophoridae: Blastobasinae]

cubensis Busck, 1934 (Ethmia); Entomologica Americana (n.s.) 13: 164. Holotype $\sigma^{\star}$, Cuba, Sierra Maestra, 1000', 28 April 1930, O. Querci. USNM Type No. 44138, Genitalia Slide AB Apr. 1932. [Elachistidae: Ethmiinae]

culminis Clarke, 1986 (Asymphorodes); Smithsonian Contributions to Zoology 416: 247. Holotype + , Marquesas Archipelago, Hiva Oa, Mt. Feani, 3800', 20 February 1968, J. F. G. \& T. M. Clarke. USNM Type No. 100804, USNM Genitalia Slide No. 24973. [Cosmopterigidae: Cosmopteriginae]

cuneatus Clarke, 1986 (Asymphorodes); Smithsonian Contributions to Zoology 416: 210. Holotype $\sigma^{*}$, Marquesas Archipelago, Fatu Hiva, Tahuna, 2000', 27 March 1968, J. F. G. \& T. M. Clarke. USNM Type No. 100785, USNM Genitalia Slide No. 24726. [Cosmopterigidae: Cosmopteriginae]

cuniculator Busck, 1914 (Batrachedra); Insecutor Inscitiae Menstruus 2: 106. Syntypes (2 $\%$ ), Hawaii, 24 July 1913. USNM Type No. 18432. Two specimens double-mounted on same block, one with abdomen lacking. [Batrachedridae]

cupreata Dognin, 1905 (Eumimographe); Annales de la Société Entomologique de Belgique 49: 87. Holotype ${ }^{\star}$, Ecuador, environs de Loja [no date]. USNM Type No. 32346, USNM Genitalia Slide No. 14481. [Oecophoridae: Oecophorinae] 

Holotype o*, Panama, Trinidad River, June 1912, A. Busck. USNM Type No. 15823. [Gelechiidae: Gelechiinae]

curvatella Busck, 1914 (Parastega); Proceedings of the U.S. National Museum 47: 16. Holotype ${ }^{*}$, Panama, Trinidad River, June 1912, A. Busck. USNM Type No. 15831, USNM Genitalia Slide No. 10669. [Gelechiidae: Gelechiinae]

curvilinella Beutenmüller, 1889 (Depressaria); Entomologica Americana 5: 10. Holotype + , USA, New York [no date]. USNM Type No. 441. Abdomen lacking. [Elachistidae: Depressariinae]

curviliniella Busck, 1914 (Catarata); Proceedings of the U.S. National Museum 47: 37. Holotype ${ }^{\star}$, Panama, Trinidad River, March 1912, A. Busck. USNM Type No. 16706, USNM Genitalia Slide No. 13239. [Elachistidae: Stenomatinae]

curvula Clarke, 1978 (Irenia); Smithsonian Contributions to Zoology 273: 44. Holotype \%, Chile, Arauco, Caramavida, 1-6 January 1954, L. E. Peña. USNM Type No. 73715, USNM Genitalia Slide No. 24277. [Oecophoridae: Oecophorinae]

cushmani Clarke, 1942 (Filatima); Proceedings of the U.S. National Museum 92: 273. Holotype $\sigma^{x}$, USA, Washington, Pullman, 24 July 1933, J. F. G. Clarke. USNM Type No. 56274, USNM Genitalia Slide No. 8771. [Gelechiidae: Gelechiinae]

cyathopoides Clarke, 1951 (Coptotelia); Acta Zoologica Lilloana 11: 344. Holotype $\sigma^{*}$, Ecuador, Zamora, March 1886. USNM Type No. 61117, USNM Genitalia Slide No. 69423. [Oecophoridae: Oecophorinae]

cyclella Busck, 1903 (Anacampsis); Proceedings of the U.S. National Museum 25: 848. Holotype $\sigma^{\star}$, USA, Arizona, Santa Rita Mountains, June, E. A. Schwarz. USNM Type No. 6371, USNM Genitalia Slide No. 10657. [Gelechiidae: Gelechiinae]

daduchus Hodges, 1966 (Batrachedra); Transactions of the American Entomological Society 92: 632. Holotype + , Jamaica, St. Andrews Parish, Hardware Gap, Dicks Pond, 25 July 1962, R. Farr \& O. Flint. USNM Type No. 67866, USNM Genitalia Slide No. 5178. [Batrachedridae]

daedala Hodges, 1964 (Sorhagenia); Proceedings of the U.S. National Museum 115: 314. Holotype $0^{\star}$, USA, California, Siskiyou Co., Mt. Shasta City, 4 July 1958, J. A. Powell. USNM Genitalia Slide No. 4771, USNM Wing Slide No. 4772. [Cosmopterigidae: Chrysopeleiinae] 
dagnirella Kaila, 1999 (Elachista); Acta Zoologica Fennica 211: 15. Holotype ${ }^{\star}$, USA, USNM Genitalia Slide No. 90100. [Elachistidae: Elachistinae]

dammersi Clarke, 1947 (Agonopterix); Journal of the Washington Academy of Sciences 37: 4. Holotype o , USA, California, San Bernardino Co., Forest Home, r.f. Senecio douglasii, C. Dammers. USNM Type No. 58012, USNM Genitalia Slide No. 148. Left hindwing in gelatin capsule. [Elachistidae: Depressariinae]

dammersi Keifer, 1936 (Gelechia); Monthly Bulletin, Department of Agriculture, California 25: 242. Holotype $\sigma^{*}$, USA, California, Palm Springs [no date]. USNM Genitalia Slide No. 7695. [Gelechiidae: Gelechiinae]

davisella Powell, 1973 (Ethmia); Smithsonian Contributions to Zoology 120: 115. Holotype $\sigma^{*}$, Mexico, Tamaulipas, 6 mi S of Ciudad Victoria, 1050', 6 August 1963, W. D. Duckworth \& D. R. Davis. [Elachistidae: Ethmiinae]

davisi Hodges, 1999 (Chionodes); Moths of America north of Mexico, fasc. 7.6: 78. Holotype $\sigma^{*}$, USA, Arizona, Cochise Co., Chiricahua Mountains, Southwest Research Station, 5400', 5-8 July 1964, D. R. Davis. [Gelechiidae: Gelechiinae]

davisi Park \& Hodges, 1995 (Dichomeris); Insecta Koreana 12: 35. Holotype ox, Taiwan, Taipei Co., Sirin, 19 September 1933, S. Issiki. USNM Genitalia Slide No. 11584. [Gelechiidae: Dichomeridinae]

decoctor Hodges, 1966 (Batrachedra); Transactions of the American Entomological Society 92: 628. Holotype ${ }^{*}$, USA, Florida, Highlands Co., Parker Islands, 26-29 May 1964, R. W. Hodges. USNM Type No. 67867, USNM Genitalia Slide No. 5173. [Batrachedridae]

decorosa Diakonoff, 1968 (Lecithocera); Bulletin of the U.S. National Museum 257 (1967): 141. Holotype +, Philippine Islands, Luzon, Mt. Makiling, [no date], P. J. Baker. USNM Genitalia Slide No. 89970. [Lecithoceridae]

decorosella Busck, 1908 (Brachyloma); Proceedings of the Entomological Society of Washington 10: 111. Holotype + , USA, New Jersey, Montclair, r.f. Quercus, em. August 1921, W. D. Kearfott. USNM Type No. 11945, W. D. Duckworth Genitalia Slide No. 2083. [Elachistidae: Stenomatinae] 
deflecta Busck, 1909 (Dichomeris); Proceedings of the Entomological Society of Washington 11: 91. Holotype 9 , USA, Arizona, Redington [no date]. USNM Type No. 12278, USNM Genitalia Slide No. 10646, USNM Wing Slide No. 9154. [Gelechiidae: Dichomeridinae]

delector Hodges, 1966 (Chedra); Transactions of the American Entomological Society 92: 640. Holotype ${ }^{\star}$, Chile, Angol, 30 January 1941, on sedge seed, D. S. Bullock, lot no. 416913. USNM Type No. 67870, USNM Genitalia Slide No. 5185. [Batrachedridae]

delitor Hodges, 1999 (Chionodes); Moths of America north of Mexico, fasc. 7.6: 83. Holotype $\sigma^{\star}$, USA, Texas, Brewster Co., Chisos Mountains, K Bar Ranch, 1 June 1973, R. W. Hodges. [Gelechiidae: Gelechiinae]

delliella Fernald, 1891 (Psecadia); Canadian Entomologist 23: 29. Holotype + , USA, Texas, [no date], Fernald Collection. [Elachistidae: Ethmiinae]

delotella Busck, 1909 (Dichomeris); Proceedings of the Entomological Society of Washington 11: 90. Holotype ${ }^{\circ}$, USA, Arizona, Baboquivari Mountains [no date]. USNM Type No. 12265, USNM Genitalia Slide No. 10644. [Gelechiidae: Dichomeridinae]

demas Busck, 1911 (Stenoma); Proceedings of the U.S. National Museum 40: 223. Holotype $\sigma^{*}$, French Guiana, St. Jean, Maroni River, [no date], W. Schaus. USNM Type No. 13588, USNM Genitalia Slide No. 13780. [Elachistidae: Stenomatinae]

denotata Bottimer, 1926 (Isophrictis similella); Journal of Agricultural Research 33: 808. Holotype ${ }^{*}$, USA, Texas, Liberty, r.f. Helianthis mollis, September 1923. USNM Type No. 27334, USNM Genitalia Slide No. 5620. [Gelechiidae: Gelechiinae]

dentata Adamski \& Brown, 2001 (Glyphidocera); Proceedings of the Entomological Society of Washington 103: 991. Holotype ${ }^{\star}$, Venezuela, Territorio Federal Amazona, Cerro de la Neblina, base camp, $140 \mathrm{~m}, 0^{\circ} 50^{\prime} \mathrm{N}, 66^{\circ} 9^{\prime} \mathrm{W}, 4-12$ February 1984, D. Davis \& T. McCabe. USNM Genitalia Slide No. 81641. [Glyphidoceridae]

dentata Diakonoff \& Sattler, 1966 (Ethmia); Entomologische Berichten 26: 189. Holotype $\uparrow$, Philippine Islands, Mindanao, Zamboanga del Sur, 30 July 1958, light trap, H. E. Milliron. USNM Genitalia Slide No. 89963. [Elachistidae: Ethmiinae]

dentella Busck, 1903 (Gelechia); Proceedings of the U.S. National Museum 25: 862. Lectotype $\sigma^{7}$, USA, Arizona, Phoenix, August, Kunze. USNM Type No. 6376, USNM Genitalia Slide No. 8054. Designated by Hodges (1999). [Gelechiidae: Gelechiinae] 
dentella Hodges, 1978 (Periploca); Moths of America north of Mexico, fasc. 6.1: 111. Holotype $\sigma^{\star}$, California, Yosemite National Park, 23 May 1941, r.f. Libocedrus decurrens, E. Ernest. USNM Genitalia Slide No. 4406. Specimen in glass vial. [Cosmopterigidae: Chrysopeleiinae]

deodora Clarke, 1962 (Brachmia); Entomological News 73: 98. Holotype ${ }^{*}$, Japan, Honsyu, Kinki, Sakai, r.f. Cedrus deodara, em. 2 July 1957, T. Kodama. USNM Type No. 65831, J. F. G. Clarke Slide No. 10726. [Gelechiidae: Gelechiinae]

deoia Hodges, 1966 (Lita); Proceedings of the U.S. National Museum 119: 20. Holotype o*, USA, California, Tulare Co., Smokey Valley, 6000', 28 September 1946, C. Henne. USNM Type No. 67645, USNM Genitalia Slide No. 7474. [Gelechiidae: Gelechiinae]

depuratella Busck, 1909 (Gelechia); Proceedings of the Entomological Society of Washington 11: 177. Holotype 9 , USA, New Mexico, Mesilla, [no date], C. N. Ainslie. USNM Type No. 12788, USNM Genitalia Slide No. 8778. [Gelechiidae: Gelechiinae]

determinata Clarke, 1964 (Gonionota); Proceedings of the U.S. National Museum 115: 68. Holotype ${ }^{*}$, British Guiana, Mallali [no date]. USNM Type No. 66374, USNM Genitalia Slide No. 68984. [Oecophoridae: Oecophorinae]

devia Hodges, 1969 (Periploca); Smithsonian Contributions to Zoology 18: 5. Holotype ^, USA, Arizona, Coconino Co., Fort Valley, 7.5 mi NW Flagstaff, 7350', 22 June 1961, R. W. Hodges. USNM Type No. 69781, USNM Genitalia Slide No. 4358. [Cosmopterigidae: Chrysopeleiinae]

diacrita Diakonoff, 1968 (Atasthalistis); Bulletin of the U.S. National Museum 257 (1967): 152. Holotype $\sigma^{\star}$, Philippine Islands, Luzon, Mt. Makiling, [no date], P. J. Baker. USNM Genitalia Slide No. 89975. [Gelechiidae: Dichomeridinae]

dialis Hodges, 1966 (Lita); Proceedings of the U.S. National Museum 119: 21. Holotype ๔, USA, Arizona, Cochise Co., Paradise, March. USNM Type No. 67646, USNM Genitalia Slide No. 7475. [Gelechiidae: Gelechiinae]

diamphidius Clarke, 1986 (Asymphorodes); Smithsonian Contributions to Zoology 416: 194. Holotype $\sigma^{7}$, T. M. Marquesas Archipelago, Hiva Oa, Mt. Feani, 3400' (1036 m), 1 March 1968, J. F. G. \& T. M. Clarke. USNM Type No. 100777. [Cosmopterigidae: Cosmopteriginae] 

Holotype $\sigma^{*}$, Marquesas Archipelago, Nuku Hiva, Tapuaooa, 2500', 30 January 1968, J. F. G. \& T. M. Clarke. USNM Type No. 100827, USNM Genitalia Slide No. 24966. [Cosmopterigidae: Cosmopteriginae]

diatriba Walsingham, 1913 (Stenoma); Biologia Centrali-Americana, Lepidoptera Heterocera 4: 171. Holotype + , Guatemala, Alta Vera Paz, Cacao, Trece Aguas, 1906, E. A. Schwarz \& H. Barber. USNM Genitalia Slide No. 14128. [Elachistidae: Stenomatinae]

dietziella Busck, 1903 (Paltodora); Proceedings of the U.S. National Museum 25: 777. Holotype + , USA, Colorado, Denver [printed on label], Chimney Gulch [hand written on label], 13 June 1898. USNM Type No. 6346, USNM Genitalia Slide No. 5601. [Gelechiidae: Gelechiinae]

difficilisella Chambers, 1872 (Evagora); Canadian Entomologist 4: 66. Holotype o*, USA, Kentucky [no date]. USNM Type No. 444, USNM Genitalia Slide No. 10676. [Gelechiidae: Gelechiinae]

diffidentia Clarke, 1986 (Asymphorodes); Smithsonian Contributions to Zoology 416: 246. Holotype + , Marquesas Archipelago, Fatu Hiva, Tahuna, 2000', 27 March 1968, J. F. G. \& T. M. Clarke. USNM Type No. 100803. [Cosmopterigidae: Cosmopteriginae]

digitella Adamski \& Brown, 2001 (Glyphidocera); Proceedings of the Entomological Society of Washington 103: 977. Holotype $\diamond^{\star}$, Venezuela, Territorio Federal Amazona, San Carlos de Rio Negro, $62^{\circ} 03^{\prime} \mathrm{N}, 62^{\circ} 03^{\prime} \mathrm{W}, 6-12$ December 1984, R. Brown. USNM Genitalia Slide No. 81459. [Glyphidoceridae]

dimorpha Busck, 1914 (Petrochroa); Insecutor Inscitiae Menstruus 2: 105. Syntypes (3 $\sigma^{\star}$, 1 \%), Hawaiian Islands, Honolulu, Oahu, O. H. Swezey. USNM Type No. 18431, ơ and $q$ genitalia on Genitalia Slide AB Nov. 22, 1930. The syntypes are mounted on a large cork block; the two undissected specimens lack abdomens. [Elachistidae: Elachistinae]

dimorpha Duckworth, 1962 (Cerconota); Proceedings of the Entomological Society of Washington 64: 39. Holotype $\sigma^{*}$, Ecuador, Guayas Province, "Eugenia" farm, kilometer 4, Canton Milagro, 1 June 1960, larva leaf-tier on Cacao. USNM Genitalia Slide No. 13000. [Elachistidae: Stenomatinae]

dimorphella Busck, 1907 (Glyphidocera); Journal of the New York Entomological Society 15: 136. Holotype $\sigma^{*}$, USA, Maryland, Montgomery Co., Plummers Island, July 1903, A. Busck. USNM Type No. 10320, USNM Genitalia Slide No. 10367. [Glyphidoceridae] 
dimorphella Clarke, 1941 (Agonopterix); Proceedings of the U.S. National Museum 90: 97. Holotype o*, USA, Illinois, Hanry, 21 May 1938, r.f. Amorpha fruticosa, M. O. Glenn. USNM Type No. 52947. [Elachistidae: Depressariinae]

discalis Busck, 1914 (Stenoma); Proceedings of the U.S. National Museum 47: 46. Holotype ${ }^{*}$, Panama, Trinidad River, March 1912, A. Busck. USNM Type No. 16722, USNM Genitalia Slide No. 12732. [Elachistidae: Stenomatinae]

disetosella Park, 1995 (Hypatima); Tropical Lepidoptera 6: 75. Holotype ־*, Taiwan, Nantou Co., Lienhuachi Forest Station, 15 km SW Puli, 250 m, 22-26 May 1980, D. Davis. USNM Genitalia Slide No. 87371. [Gelechiidae: Dichomeridinae]

dispersa Duckworth, 1967 (Lethata); Proceedings of the U.S. National Museum 122: 13. Holotype ${ }^{\star}$, Brazil, Matto Grosso, Chapada, near Cuyaba, [no date], H. H. Smith. USNM Genitalia Slide No. 13385. [Elachistidae: Stenomatinae]

dissimilis Kearfott, 1911 (Antaeotricha); Entomological News 22: 126. Holotype ơ, Brazil, São Paulo [no date]. USNM Genitalia Slide No. 17757. [Elachistidae: Stenomatinae]

dissita Clarke, 1964 (Gonionota); Proceedings of the U.S. National Museum 115: 67. Holotype + , Trinidad, Port of Spain, [no date], F. W. Urich. USNM Type No. 66372, USNM Genitalia Slide No. 68948. [Oecophoridae: Oecophorinae]

dissona Kaila, 1997 (Elachista); Acta Zoologica Fennica 206: 14. Holotype ${ }^{\star}$, USA, Colorado, Alamosa Co., Sand Dunes Staff Quarters, 8200', pinyon-juniper zone, 24 June 1982, R. W. Hodges. USNM Genitalia Slide No. 89290. [Elachistidae: Elachistinae]

distinctella Dietz, 1910 (Blastobasis); Transactions of the American Entomological Society 36: 10. Holotype ${ }^{*}$, USA [no other data]. USNM Genitalia Slide No. 80988. [Coleophoridae: Blastobasinae]

diva Hodges, 1986 (Dichomeris); Moths of America north of Mexico, fasc. 7.1: 570. Holotype ${ }^{\star}$, USA, Arizona, Santa Cruz Co., 1 mi S Patagonia, 29-30 July 1964, D. R. Davis. USNM Genitalia Slide No. 9114. [Gelechiidae: Dichomeridinae]

diveni Heinrich, 1921 (Borkhausenia); Journal of Agricultural Research 20: 814. Holotype ^, USA, Texas, Brownsville, r.f. Lantana horrida, [no date], E. L. Diven. USNM Type No. 23741, Heinrich Genitalia Slide 7 Nov 1919. [Cosmopterigidae: Cosmopteriginae] 
diversella Busck, 1916 (Gelechia); Proceedings of the Entomological Society of Washington 18: 149. Holotype 9 , USA, California, San Diego Co., 28 May 1911, W. S. Wright. USNM Type No. 20069, USNM Genitalia Slide No. 7561. [Gelechiidae: Gelechiinae]

dives Dietz, 1910 (Holcocera); Transactions of the American Entomological Society 36: 26. Lectotype $\sigma^{*}$, USA, Massachusetts, Cohasset, 16 June 1907, O. Bryant. USNM Genitalia Slide No. 81004. Designated by Adamski \& Hodges (1996). [Coleophoridae: Blastobasinae]

docilis Diakonoff, 1968 (Lecithocera); Bulletin of the U.S. National Museum 257 (1967): 144. Holotype $\sigma^{*}$, Philippine Islands, Luzon, Mt. Makiling, [no date], P. J. Baker. USNM Genitalia Slide No. 89972. [Lecithoceridae]

dolo Hodges, 1999 (Chionodes); Moths of America north of Mexico, fasc. 7.6: 198. Holotype $o^{*}$, USA, Canada, Yukon Territory, Dempster Highway, km 155, Ogilvie Mountains, 1050 m, 4 July 1985, K. Mikkola. [Gelechiidae: Gelechiinae]

dorsalis Busck, 1914 (Anacampsis); Proceedings of the U.S. National Museum 47: 8. Syntype ${ }^{*}$, Panama, Trinidad River, June 1912, A. Busck. USNM Type No. 15819. Abdomen and right fore- and hindwing lacking. [Gelechiidae: Gelechiinae]

dracunculi Clarke, 1933 (Depressaria artemisiae); Canadian Entomologist 65: 90. Holotype $\sigma^{*}$, USA, Washington, Whitman Co., Snake River, opposite Clarkston, 20 April 1931, J. F. G. Clarke. USNM Type No. 44743. [Elachistidae: Depressariinae]

drapeta Hodges, 1999 (Chionodes); Moths of America north of Mexico, fasc. 7.6: 63. Holotype ${ }^{*}$, USA, Florida, Monroe Co., Key Largo, 11 November 1967, S. Kemp. [Gelechiidae: Gelechiinae]

drimyla Diakonoff, 1968 (Frisilia); Bulletin of the U.S. National Museum 257 (1967): 128. Holotype + , Philippine Islands, Luzon, Mt. Makiling, [no date], P. J. Baker. USNM Genitalia Slide No. 89995. Left fore- and hindwing lacking. [Lecithoceridae]

dromicella Busck, 1910 (Gelechia); Proceedings of the Entomological Society of Washington 11: 177. Holotype 9 , USA, California, Placer Co., September, A. Koebele. USNM Type No. 12792, USNM Genitalia Slide No. 6762. [Gelechiidae: Gelechiinae]

dryocrypta Meyrick, 1931 (Hypercallia); Exotic Microlepidoptera 4: 121. Syntype + , Guatemala, Cayuga, April, W. Schaus. USNM Genitalia Slide No. 68983. Described from two females. [Oecophoridae: Oecophorinae] 
duckworthi Powell, 1973 (Ethmia); Smithsonian Contributions to Zoology 120: 165. Holotype ${ }^{\star}$, Panama, Barro Colorado Island, 1-9 May 1964, W. D. \& S. S. Duckworth. USNM Genitalia Slide No. 89952. [Elachistidae: Ethmiinae]

dudiella Busck, 1903 (Gnorimoschema); Proceedings of the U.S. National Museum 25: 828. Syntypes (2 ${ }^{\circ}$ ), USA, Arizona, Williams, June 1901, H. S. Barber. USNM Type No. 6361, USNM Genitalia Slide No. 6961. [Gelechiidae: Gelechiinae]

dulcinella Kaila, 1999 (Elachista); Acta Zoologica Fennica 211: 95. Holotype ॰ ${ }^{\star}$ USA, Florida, Palmdale, Fisheating Creek, 7-10 May 1964, R. W. Hodges. USNM Genitalia Slide No. 90102. [Elachistidae: Elachistinae]

dyariella Busck, 1903 (Gelechia); Proceedings of the U.S. National Museum 25: 877. Syntypes (6), USA, Colorado, [no date], Dyar. USNM Type No. 6388, USNM Genitalia Slide No. 6763. [Gelechiidae: Gelechiinae]

echinopanicia Clarke, 1941 (Agonopterix rosaciliella); Proceedings of the U.S. National Museum 90: 86. Holotype ${ }^{*}$, USA, Washington, Skyline Ridge, Mt. Baker District, r.f. Echinopanax horridum, 5 September 1933, J. F. G. Clarke. USNM Type No. 52252, USNM Genitalia Slide No. 170. [Elachistidae: Depressariinae]

ectopon Hodges, 1986 (Helcystogramma); Moths of America north of Mexico, fasc. 7.1: 131. Holotype ${ }^{*}$, USA, Nebraska, Cherry Co., Fort Niobrara NWR, 29 June 1983, R. W. Hodges. USNM Genitalia Slide No. 12111. [Gelechiidae: Dichomeridinae]

edax Hodges, 1962 (Ithome); Journal of the Lepidopterists' Society 15: 88. Holotype $o^{*}$, USA, Texas, Brownsville, r.f. blossoms of ebony (Byra sp.), em. 7 June 1944. USNM Genitalia Slide No. 4848. [Cosmopterigidae: Chrysopeleiinae]

edithella Barnes \& Busck, 1920 (Psacaphora); Contributions to the Natural History of the Lepidoptera of North America 4: 222. Holotype o*, USA, Colorado, Golden, Chimney Gulch, 7 June 1907, Oslar. USNM Type No. 10323, USNM Genitalia Slide No. 1012. Abdomen in gelatin capsule. [Coleophoridae: Momphinae]

edithella Busck, 1907 (Epicallima); Journal of the New York Entomological Society 15: 138. Holotype ${ }^{*}$, USA, New Hampshire, Center Harbor, [no date], H. Dyar. USNM Type No. 10323, USNM Genitalia Slide No. 1012. [Oecophoridae: Oecophorinae]

edulicola Hodges \& Stevens, 1978 (Coleotechnites); Journal of the Lepidopterists' Society 32: 120. Holotype $\sigma^{\star}$, USA, New Mexico, 14 km N Aztec, r.f. Pinus edulis, June 1973, R. Stevens. USNM Type No. 75472. [Gelechiidae: Gelechiinae] 

Holotype $\sigma^{\star}$, USA, Texas, 17 May 1904, H. Barber. USNM Type No. 9769, USNM Genitalia Slide No. 6262. [Gelechiidae: Gelechiinae]

elainae Hodges, 1999 (Chionodes); Moths of America north of Mexico, fasc. 7.6: 192. Holotype $\sigma^{*}$, USA, Arizona, Cochise Co., Chiricahua Mountains, Onion Saddle, 7600', 1 July 1967, J. G. Franclemont. [Gelechiidae: Gelechiinae]

eleanorae Clarke, 1941 (Depressaria); Proceedings of the U.S. National Museum 90: 178. Holotype $\alpha^{\star}$, Canada, Ontario, Hymers, 16-23 August. USNM Type No. 52082. [Elachistidae: Depressariinae]

elegans Hodges, 1978 (Walshia); Moths of America north of Mexico, fasc. 6.1: 124. Holotype o*, USA, Louisiana, Tangipahoa Parish, Fluker, 13 September 1971, G. Strickland. USNM Genitalia Slide No. 224 [Cosmopterigidae: Chrysopeleiinae]

elegans Park, 2001 (Dichomeris); Insecta Koreana 18: 308. Holotype $\odot^{\star}$, Taiwan, Pingtung Co., Kenting Park, 50 m, 29-31 August 1983, J. B. Heppner. USNM Genitalia Slide No. 87441. Specimen not found. [Gelechiidae: Dichomeridinae]

elena Clarke, 1951 (Coptotelia); Acta Zoologica Lilloana 11: 349. Holotype + , Brazil, Amazones, São Paulo de Olivença, November-December, Fassel. USNM Type No. 61119, USNM Genitalia Slide No. 69435. [Oecophoridae: Oecophorinae]

elephas Walsingham, 1910 (Anacampsis); Biologia Centrali-Americana, Lepidoptera Heterocera 4: 39. Holotype ${ }^{\star}$, Guatemala, San Geronimo, Champion, Walsingham No. 65726. [Gelechiidae: Gelechiinae]

elongata Moriuti, 1963 (Lycophantis); Kontyû 31: 266. Holotype 9 , Formosa [Taiwan], Marrepa, 13 August 1943, A. Mutuura. Specimen not found. [Oecophoridae: Oecophorinae]

elpista Walsingham, 1911 (Glyphidocera); Biologia Centrali-Americana, Lepidoptera Heterocera 4: 110. Holotype + , Panama, Tabernilla, Canal Zone, [no date], A. Busck. Abdomen in gelatin capsule. [Glyphidoceridae]

elucidella Barnes \& Busck, 1920 (Eucordylea); Contributions to the Natural History of the Lepidoptera of North America 4: 227. Holotype $\diamond^{*}$, USA, California, San Diego Co., San Diego, June. USNM Genitalia Slide No. 6564. [Gelechiidae: Gelechiinae] 
elutella Busck, 1914 (Ethmia); Proceedings of the U.S. National Museum 47: 35. Holotype 9 , Panama, Porto Bello, March 1911, A. Busck. USNM Type No. 16697. [Elachistidae: Ethmiinae]

emma Busck, 1911 (Gonioterma); Proceedings of the U.S. National Museum 40: 226. Holotype $q$, French Guiana, St. Jean, Maroni River, [no date], W. Schaus. USNM Type No. 13594, USNM Genitalia Slide No. 14126. [Elachistidae: Stenomatinae]

emmeli Powell, 1973 (Ethmia monticola); Smithsonian Contributions to Zoology 120: 106. Holotype ${ }^{*}$, USA, Arizona, Coconino Co., Fort Valley, 7.5 mi NW Flagstaff, 7350', 27 June 1961, R. W. Hodges. [Elachistidae: Ethmiinae]

emphereia Clarke, 1986 (Asymphorodes); Smithsonian Contributions to Zoology 416: 237. Holotype ${ }^{\star}$, Marquesas Archipelago, Hiva Oa, Mt. Feani, 2400', 1 March 1968, J. F. G. \& T. M. Clarke. USNM Type No. 100795, USNM Genitalia Slide No. 24826. [Cosmopterigidae: Cosmopteriginae]

emptor Hodges, 1999 (Chionodes); Moths of America north of Mexico, fasc. 7.6: 62. Holotype $\sigma^{\star}$, USA, Florida, Highlands Co., Archbold Biological Station, Lake Placid, 8-15 May 1964, R. W. Hodges. [Gelechiidae: Gelechiinae]

empusa Hodges, 1986 (Dichomeris); Moths of America north of Mexico, fasc. 7.1: 59. Holotype $\sigma^{*}$, USA, Arizona, Coconino Co., West Fork, 16 mi SW Flagstaff, 6500', 4 July 1961, R. W. Hodges. USNM Genitalia Slide No. 9125. [Gelechiidae: Dichomeridinae]

enchrysa Hodges, 1962 (Eteobalea); Entomologica Americana 42: 72. Holotype ơ, USA, California, San Bernardino Co., Cajon Valley, r.f. Trichostoma lanatum, em. 30 June 1938, C. Dammers. USNM Type No. 66082. Right fore- and hindwing lacking. [Cosmopterigidae: Cosmopteriginae]

engelella Busck, 1906 (Mompha); Canadian Entomologist 38: 123. Syntypes (1 $0^{\star}, 1$ ), USA, Washington, D.C., June 1902, A. Busck; USA, Pennsylvania, Pittsburgh, 29 May 1905. USNM Type No. 9797. Syntypes not found, but there are 6 specimens from Pittsburgh (none from 29 May 1905) with red “Type” labels. [Coleophoridae: Momphinae]

ensis Hodges, 1999 (Chionodes); Moths of America north of Mexico, fasc. 7.6: 216. Holotype ${ }^{\circ}$, USA, Utah, Sanpete Co., head of Ephraim Canyon, 10,000-10,300', 28 July 1981, R. W. Hodges. USNM Genitalia Slide No. 11956. [Gelechiidae: Gelechiinae] 
entoloma Busck, 1913 (Coleophora); Journal of Entomology and Zoology (Pomona College) 5: 97. Syntypes (2ð), USA, California, San Diego, 1-7 June and 16-23 June, W. S. Wright. USNM Type No. 15608. Specimens not found. [Coleophoridae: Coleophorinae]

ephialtes Walsingham, 1911 (Tabernillaia); Biologia Centrali-Americana, Lepidoptera Heterocera 4: 54. Holotype $\&$, Panama, Canal Zone, Tabernilla, June 1907, A. Busck, Walsingham No. 5850. Abdomen glued to double-mount block. [Gelechiidae: Gelechiinae]

epileuca Wu \& Park, 1999 (Kalocyrma); Insecta Koreana 16: 136. Holotype ^, Sri Lanka, Rat. District, Uggalkaltota, Irrigation Bungalow, 350', 31 January-8 February 1970, D. Davis \& B. Rowe. USNM Genitalia Slide No. 88985. [Lecithoceridae]

epilygella Powell, 1973 (Ethmia); Smithsonian Contributions to Zoology 120: 137. Holotype $o^{\star}$, Brazil, Nova Teutonia, 300-500 m, $27^{\circ} 11^{\prime} \mathrm{S}, 52^{\circ} 23^{\prime} \mathrm{W}$, August 1963, F. Plaumann. [Elachistidae: Ethmiinae]

eremia Clarke, 1971 (Gonionota); Smithsonian Contributions to Zoology 95: 16. Holotype o, French Guiana, Cayenne, [no date], W. Schaus. USNM Type No. 71093, USNM Genitalia Slide No. 68756. [Oecophoridae: Oecophorinae]

eremnogramma Clarke, 1978 (Doina); Smithsonian Contributions to Zoology 273: 30. Holotype ${ }^{\star}$, Chile, Arauco, Caramavida, 1-6 January 1954, L. E. Peña. USNM Type No. 73707, USNM Genitalia Slide No. 24259. [Amphisbatidae]

eremonoma Braun, 1948 (Onceroptila); Memoirs of the American Entomological Society 13: 12. Holotype ox, USA, Utah, Eureka, 9 August 1911, T. Spalding. USNM Type No. 34825, USNM Genitalia Slide No. 10716. [Elachistidae: Elachistinae]

eremos Hodges, 1969 (Perimede); Smithsonian Contributions to Zoology 18: 12. Holotype , USA, Arkansas, Washington Co., Devil's Den State Park, 27 June 1966, R. W. Hodges. USNM Type No. 69788, USNM Genitalia Slide No. 4800. [Cosmopterigidae: Chrysopeleiinae]

eriobotryae Busck, 1915 (Blastobasis); Proceedings of the Entomological Society of Washington 17: 85. Lectotype of, USA, Florida, Miami, r.f. loquat (Eriobotrya japonica), July 1910. USNM Type No. 19233. Designated by Adamski \& Hodges (1996). [Coleophoridae: Blastobasinae] 
eriobotryae Busck, 1939 (Proclesis); Anales de la Sociedad Cientifica de Argentina 127: 361. Holotype $\diamond^{\star}$, Argentina, Tucumán, r.f. Eriobotrya japonica [no date]. USNM Type No. 53116. [Deoclonidae]

eriogonella Clarke, 1935 (Gelechia); Canadian Entomologist 67: 247. Holotype ${ }^{\star}$, USA, Washington, Whitman Co., Pullman, r.f. Eriogonum heraclioides, em. 19 July 1934, J. F. G. Clarke. USNM Type No. 50943, USNM Genitalia Slide No. 9920. [Gelechiidae: Gelechiinae]

erransella Chambers, 1874 (Perimede); Canadian Entomologist 6: 52. Pseudotypes (2\%), USA, Kentucky [no date]. USNM Type No. 492, USNM Genitalia Slide No. 4845. Both specimens have handwritten Chambers labels and one has a USNM red type label. Miller and Hodges (1990) indicate that the holotype is in MCZ. [Cosmopterigidae: Chrysopeleiinae]

eryngiella Bottimer, 1926 (Recurvaria); Journal of Agricultural Research 33: 812. Holotype $\sigma^{*}$, USA, Texas, Chambers Co., Stowell, r.f. Eryngium aquaticum, July 1923. USNM Type No. 27336, USNM Genitalia Slide No. 6234. [Gelechiidae: Gelechiinae]

erythropennis Dognin, 1913 (Dasycera); Annales de la Société Entomologique de Belgique 57: 416. Holotype $\diamond^{\star}$, Colombia, November, Fassel, Dognin Collection. USNM Type No. 32360, USNM Genitalia Slide No. 77914. According to the original description, the type was collected in July. [Elachistidae: Stenomatinae]

eschara Clarke, 1978 (Revonda); Smithsonian Contributions to Zoology 273: 42. Holotype $\sigma^{*}$, Chile, Arauco, Cordillera de Nahuelbuta, Pichinahuel, 1100-1400 m, 23-31 January 1954, L. E. Peña. USNM Type No. 71713, USNM Wing Slide No. 24194. Abdomen lacking. [Oecophoridae: Oecophorinae]

esor Hodges, 1999 (Chionodes); Moths of America north of Mexico, fasc. 7.6: 63. Holotype o*, USA, Florida, Monroe Co., Big Pine Key, 19 May 1988, ex-larva on Borrichia arborescens flower head, em. 29 June 1988, D. H. Habeck. [Gelechiidae: Gelechiinae]

eupatoriella Busck, 1934 (Aristotelia); Entomologica Americana 13: 159. Holotype o , Cuba, Santiago de las Vegas, r.f. Eupatorium [no date]. Genitalia Slide J. F. G. Clarke 10975. [Gelechiidae: Gelechiinae]

euryaula Meyrick, 1925 (Coleophora); Bulletin de la Société Royale Entomologique d’Egypte1925: 215. Lectotype + , Egypt, [no date], A. Alfieri. USNM Genitalia Slide No. 87144. Larval case and adult affixed to double mount. Lectotype designated by Baldizzone \& Landry (1993). [Coleophoridae: Coleophorinae] 
eurysia Wu, 2000 (Philharmonia); Acta Zootaxonomica Sinica 25: 427. Holotype o*, 510 Sarawak, Semengoh Forest Reserve, 15 mi S Kuching, 16 September 1966. USNM Genitalia Slide No. 89121. [Lecithoceridae]

euzosta Walsingham, 1912 (Triclonella); Biologia Centrali-Americana, Lepidoptera Heterocera 4: 137. Holotype $\&$, Panama, Canal Zone, Tabernilla, June 1907, A. Busck. Genitalia Slide AB Oct. 5, 1935. [Cosmopterigidae: Cosmopteriginae]

evippeella Busck, 1906 (Agnippe); Proceedings of the U.S. National Museum 30: 723. Holotype $\diamond^{\star}$, USA, Texas, Brownsville, 1 May 1904, H. Barber. USNM Type No. 9766, USNM Genitalia Slide No. 10653. [Gelechiidae: Gelechiinae]

excavata Busck, 1914 (Dichomeris); Proceedings of the U.S. National Museum 47: 18. Holotype ?, Panama, Porto Bello, March 1911, A. Busck. USNM Type 16674, USNM Genitalia Slide No. 15494. [Gelechiidae: Dichomeridinae]

excavata Clarke, 1964 (Gonionota); Proceedings of the U.S. National Museum 115: 79. Holotype + , Mexico, Orizaba, [no date], W. Schaus. USNM Type No. 66378, USNM Genitalia Slide No. 68973. [Oecophoridae: Oecophorinae]

exquisita Duckworth, 1964 (Gonioterma); Proceedings of the U.S. National Museum 115: 382. Holotype ${ }^{\star}$, Brazil, Lassance, Minasgar, 9-12 November 1912, Cornell Univ. Exped. Lot 569. USNM Genitalia Slide No. 23331. [Elachistidae: Stenomatinae]

extenta Busck, 1920 (Stenoma); Insecutor Inscitiae Menstruus 8: 90. Holotype ${ }^{\star}$, Guatemala, Cayuga, [no date], W. Schaus. USNM Type No. 22311, USNM Genitalia Slide No. 17743. [Elachistidae: Stenomatinae]

extima Clarke, 1964 (Gonionota); Proceedings of the U.S. National Museum 115: 76. Holotype + , Costa Rica, Tuis, 28 May-4 June, W. Schaus. USNM Type No. 66376, USNM Genitalia Slide No. 68986. [Oecophoridae: Oecophorinae]

fabajuxta Wu \& Park, 1999 (Carodista); Insecta Koreana 16: 134. Holotype o*, Sri Lanka, NE District, Moon Plains, 1635', 18 August 1973, at black light, G. Ekis. USNM Genitalia Slide No. 89192. [Lecithoceridae]

factor Hodges, 1999 (Chionodes); Moths of America north of Mexico, fasc. 7.6: 196. Holotype + , USA, California, San Bernardino Co., Big Bear Lake, 6800', 6 August 1978, R. Leuschner. USNM Genitalia Slide No. 874. [Gelechiidae: Gelechiinae] 
facula Wu \& Park, 1999 (Torodora); Tinea 16 (1): 65. Holotype ${ }^{*}$, Sri Lanka, Rat. District, Uggalkaltota, Irrigation Bungalow, 350', 31 January-8 February, 1970, D. Davis \& B. Rowe. USNM Genitalia Slide No. 89049. [Lecithoceridae]

facunda Hodges, 1962 (Cosmopterix); Entomologica Americana (n.s.) 42: 55. Holotype 9 , USA, Texas, Brownsville, 27 March 1928, F. H. Benjamin. USNM Type No. 66081, USNM Genitalia Slide No. 3941. [Cosmopterigidae: Cosmopteriginae]

fagella Busck, 1903 (Trypanisma); Proceedings of the U.S. National Museum 25: 816. Holotype ${ }^{\star}$, USA, District of Columbia [no date]. USNM Type No. 6357, USNM Genitalia Slide No. 6594. Left fore- and hindwing lacking. [Gelechiidae: Gelechiinae]

falcata Clarke, 1978 (Utilia); Smithsonian Contributions to Zoology 273: 51. Holotype o*, Chile, Centro-Austral, January-March 1898, V. Izquerdo. USNM Type No. 73717, USNM Genitalia Slide No. 24282, USNM Wing Slide No. 24191. [Oecophoridae: Oecophorinae]

falcata Hodges, 1978 (Siskiwitia); Moths of America north of Mexico, fasc. 6.1: 112. Holotype ${ }^{*}$, USA, Florida, Archbold Biological Station, Lake Placid, 16-22 May 1964, R. W. Hodges. USNM Genitalia Slide No. 7825. [Cosmopterigidae: Chrysopeleiinae]

falculinella Busck, 1911 (Gonada); Proceedings of the U.S. National Museum 40: 211. Holotype $\sigma^{*}$, French Guiana, St. Jean, Maroni River, [no date], W. Schaus. USNM Type No. 13565, USNM Genitalia Slide No. 4070. [Oecophoridae: Oecophorinae]

fasciatum Busck, 1911 (Stenoma); Proceedings of the U.S. National Museum 40: 217. Holotype $\sigma^{*}$, French Guiana, Cayenne, [no date], W. Schaus. USNM Type No. 13575, USNM Genitalia Slide No. 12761. [Elachistidae: Stenomatinae]

fasciola Clarke, 1986 (Herlinda); Smithsonian Contributions to Zoology 416: 285. Holotype ${ }^{\star}$, Marquesas Archipelago, Fatu Hiva, Omoa, 14 March 1968, J. F. G. \& T. M. Clarke. USNM Type No. 100823, USNM Genitalia Slide No. 25122. [Cosmopterigidae: Cosmopteriginae]

fassliana Dognin, 1913 (Hilarographa); Annales de la Société Entomologique de Belgique 57: 417. Holotype o*, Colombia, San Antonio, [no date], Fassel. USNM Genitalia Slide No. 77307. [Elachistidae: Stenomatinae]

fatula Hodges, 1975 (Psilocorsis); Journal of the Lepidopterists' Society 29: 91. Holotype + , USA, Texas, Culberson Co., Guadalupe Mountains, Smith Canyon, 5750', 22 May 1973, R. W. Hodges. USNM Genitalia Slide No. 4650. [Amphisbatidae] 
faustella Busck, 1910 (Gnorimoschema); Proceedings of the Entomological Society of Washington 11: 175. Holotype 9 , USA, New Mexico, Beulah, 8000', July, T. D. A. Cockerell. USNM Type No. 12699, USNM Genitalia Slide No. 6975. [Gelechiidae: Gelechiinae]

favilla Clarke, 1986 (Asymphorodes); Smithsonian Contributions to Zoology 416: 257. Holotype $o^{\star}$, Marquesas Archipelago, Hiva Oa, Mt. Feani, 2000', 1 March 1968, J. F. G. \& T. M. Clarke. USNM Type No. 100813, USNM Genitalia Slide No. 25023. [Cosmopterigidae: Cosmopteriginae]

felisae Clarke, 1962 (Homoeoprepes); Proceedings of the U.S. National Museum 113: 375. Holotype $\sigma^{*}$, Colombia, Cauca, Paramo de Parace, Lake San Rafael, 3570 m, 27 January 1950, J. F. G. Clarke. Abdomen lacking. [Elachistidae: Agonoxeniinae]

felix Busck, 1914 (Stenoma); Proceedings of the U.S. National Museum 47: 401. Holotype †, Panama, Cabima, May 1911, A. Busck. USNM Type No. 16713, USNM Genitalia Slide No. 13928. [Elachistidae: Stenomatinae]

fenestra Busck, 1914 (Stenoma); Proceedings of the U.S. National Museum 47:44. Holotype $\sigma^{\star}$, Costa Rica, Sixola River, [no date], W. Schaus. USNM Type No. 16719, USNM Genitalia Slide No. 13876. [Elachistidae: Stenomatinae]

fernaldella Riley, 1889 (Setiostoma); Proceedings of the Entomological Society of Wash-

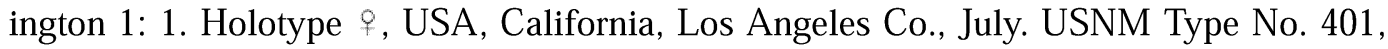
W. D. Duckworth Genitalia Slide No. 2081. [Elachistidae: Stenomatinae]

fernaldella Busck, 1903 (Trichotaphe); Proceedings of the U.S. National Museum 25: 915. Holotype $\sigma^{*}$, USA, Maine, Orono [no date]. Abdomen lacking. [Gelechiidae: Dichomeridinae]

fernaldella Walsingham, 1882 (Cosmopteryx); Transactions of the American Entomological Society 10: 97 . Holotype (uncertain sex), USA. The type-locality is unknown according to Hodges (1978). Abdomen lacking. [Cosmopterigidae: Cosmopteriginae]

fernandezyepezi Duckworth, 1967 (Lethata); Proceedings of the U.S. National Museum 122: 14. Holotype ${ }^{*}$, Venezuela, Rancho Grande, 1100 m, 16-19 January 1966, S. S. \& W. D. Duckworth. USNM Genitalia Slide No. 13386. [Elachistidae: Stenomatinae]

festiva Busck, 1914 (Ethmia); Proceedings of the U.S. National Museum 47: 33. Holotype o, Panama, Porto Bello, May 1912, A. Busck. USNM Type No. 16695. [Elachistidae: Ethmiinae] 
fictor Hodges, 1999 (Chionodes); Moths of America north of Mexico, fasc. 7.6: 219. Holotype ${ }^{\star}$, USA, Alaska, Brooks Range, Atigun Pass and below, 7 July 1979, R. Leuschner. USNM Genitalia Slide No. 15968. [Gelechiidae: Gelechiinae]

figurella Busck, 1912 (Gelechia); First Annual Report of the Laguna Marine Laboratory (Pomona College) 1912: 168. Holotype 9, USA, California, Los Angeles Co., Laguna Beach, [no date], C. F. Baker. USNM Genitalia Slide No. 9900. [Gelechiidae: Gelechiinae]

figurodigita Wu \& Park, 1998 (Deltoplastis); Insecta Koreana 15: 2. Holotype o , Sri Lanka, Gal. District, Udugama, Kanneliya Jungle, 400', 6-12 October 1973, at black light, Krombein, Karuntaratne, Fernando \& Ferdinando. USNM Genitalia Slide No. 89175. [Lecithoceridae]

fimbriata Clarke, 1964 (Gonionota); Proceedings of the U.S. National Museum 115: 82. Holotype ${ }^{\star}$, Panama, Porto Bello, April 1912, A. Busck. USNM Type No. 66379, USNM Genitalia Slide No. 68985. [Oecophoridae: Oecophorinae]

fimus Hodges, 1999 (Chionodes); Moths of America north of Mexico, fasc. 7.6: 204. Holotype ox, USA, Alaska, Schrader Lake, 9 July 1973, J. F. G. Clarke. USNM Genitalia Slide No. 14656. [Gelechiidae: Gelechiinae]

fistuca Hodges, 1986 (Dichomeris); Moths of America north of Mexico, fasc. 7.1: 68. Holotype ${ }^{\star}$, USA, South Carolina, Charleston Co., McClellanville, Wedge Plantation, 27 April 1981, R. W. Hodges. [Gelechiidae: Dichomeridinae]

flavicomella Engel, 1907 (Depressaria); Entomological News 18: 276. Lectotype ${ }^{\star}$, USA, Pennsylvania, New Brighton, 19 June 1905, H. D. Merrick. USNM Type No. 10284. Abdomen lacking. Designated by Hodges (1974). [Elachistidae: Depressariinae]

flavicorporella Walsingham, 1882 (Gelechia); Transactions of the American Entomological Society 10: 177. Lectotype $\sigma^{*}$, USA, Maine, Orono [no date]. USNM Genitalia Slide No. 87136. Designated by Hodges (1999). [Gelechiidae: Gelechiinae]

flavidella Busck, 1914 (Recurvaria); Proceedings of the U.S. National Museum 47: 12. Holotype o*, Panama, Paraiso, Isthmian Canal Zone, May 1911, A. Busck. USNM Type No. 15825, USNM Genitalia Slide No. 10664. [Gelechiidae: Gelechiinae]

fletcherella Gibson, 1909 (Psilocorsis); Ottawa Naturalist 22: 226. Holotype + , Canada, Ontario, Ottawa [no date]. [Amphisbatidae] 
flinti Clarke, 1978 (Doina); Smithsonian Contributions to Zoology 273: 24. Holotype $q$, 510 Chile, Cautin, near Pucon, 4 January 1966, O. Flint \& Cekalovic. USNM Type No. 73701. [Amphisbatidae]

flipria Hodges, 1974 (Malthildana); Moths of America north of Mexico, fasc. 6.2: 122. Holotype $\approx$, USA, North Carolina, Black Mountain, [no date], W. Beutenmüller. USNM Genitalia Slide No. 1075. [Oecophoridae: Oecophorinae]

florella Busck, 1903 (Gnorimoschema); Proceedings of the U.S. National Museum 25: 832. Holotype ${ }^{\star}$, USA, Colorado, Denver [no date]. USNM Type No. 6363. Abdomen lacking. [Gelechiidae: Gelechiinae]

floridanella Beutenmüller, 1889 (Cosmopteryx); Entomologica Americana 5: 10. Holotype ${ }^{*}$, USA, Central Florida [no date]. USNM Type No. 496. Abdomen lacking. [Cosmopterigidae: Cosmopteriginae]

floridanella Busck, 1901 (Glyphidocera); Proceedings of the Entomological Society of Washington 4: 475. Holotype $\sigma^{*}$, USA, Florida [no date]. USNM Type No. 5363, Genitalia Slide AB Dec. 16, 1920. [Glyphidoceridae]

floridella Dietz, 1910 (Valentinia); Transactions of the American Entomological Society 36: 17. Lectotype ${ }^{\star}$, USA, Florida, Crescent City, r.f. ${ }^{\star}$ cone of Zamia integrifolia, em. 4 June 1895. USNM Genitalia Slide No. 80985. Designated by Adamski \& Hodges (1996). [Coleophoridae: Blastobasinae]

floridensis Hodges, 1978 (Walshia); Moths of America north of Mexico, fasc. 6.1: 125. Holotype ${ }^{*}$, USA, Florida, Lake Alfred, 31 December 1929, r.f. Kuhnistera pinnata, L. J. Bottimer. [Cosmopterigidae: Chrysopeleiinae]

florinda Clarke, 1978 (Utilia); Smithsonian Contributions to Zoology 273: 53. Holotype ${ }^{\star}$, Chile, Araucania, 1 March 1892, V. Izquerdo. USNM Type No. 73718, USNM Genitalia Slide No. 24283. [Oecophoridae: Oecophorinae]

fluvialella Busck, 1908 (Gelechia); Canadian Entomologist 40: 194. Holotype ${ }^{\circ}$, USA, Pennsylvania, Allegheny Co., Oak Station, [no date], F. Marloff. USNM Type No. 11558, USNM Genitalia Slide No. 7891. [Gelechiidae: Gelechiinae]

folia Hodges, 1966 (Batrachedra); Transactions of the American Entomological Society 92: 601. Holotype 9 , USA, Arizona, Coconino Co., Hochderffer Hill, 12.5 mi NNW Flagstaff, 8500', 4 August 1961, R. W. Hodges. USNM Type No. 67853, USNM Genitalia Slide No. 5056. Right hindwing lacking. [Batrachedridae] 
fondella Busck, 1906 (Gelechia); Canadian Entomologist 38: 122. Holotype o*, USA, Maryland, Montgomery Co., Plummers Island [no date]. USNM Type No. 9795, USNM

Genitalia Slide No. 8273. Right fore- and hindwing lacking. [Gelechiidae: Gelechiinae]

fractura Clarke, 1986 (Asymphorodes); Smithsonian Contributions to Zoology 416: 247. Holotype $\sigma^{*}$, Marquesas Archipelago, Nuku Hiva, Tunoa Ridge, 2900', 23 January 1968, J. F. G. \& T. M. Clarke. USNM Type No. 100805. [Cosmopterigidae: Cosmopteriginae]

fragariae Busck, 1919 (Aristotelia); Proceedings of the Entomological Society of Washington 21: 52. Holotype 9 , Canada, British Columbia, Victoria [no date]. USNM Genitalia Slide No. 5886. [Gelechiidae: Gelechiinae]

fragariella Busck, 1904 (Anacampsis); Proceedings of the U.S. National Museum 27: 760. Holotype $\sigma^{\star}$, USA, Washington, Pullman, r.f. strawberry leaves (Fragaria sp.), July, Piper. USNM Type No. 7861, USNM Genitalia Slide No. 10659. [Gelechiidae: Gelechiinae]

fragmentella Dognin, 1913 (Stenoma); Annales de la Société Entomologique de Belgique 57: 417. Holotype ${ }^{*}$, French Guiana, H. Rolle, Berlin, S.W.11, Dognin Collection. USNM Genitalia Slide No. 14115, USNM Type No. 32367. [Elachistidae: Stenomatinae]

franclemonti Hodges, 1999 (Chionodes); Moths of America north of Mexico, fasc. 7.6: 65. Holotype ${ }^{\star}$, USA, New Jersey, Ocean Co., Wrangle Brook Road, 28 August 1956, J. G. Franclemont. [Gelechiidae: Gelechiinae]

fraternella Busck, 1910 (Stenoma); Proceedings of the Entomological Society of Washington 12: 80. Holotype $\sigma^{\star}$, Costa Rica, Juan Viñas, [no date], W. Schaus. USNM Type No. 13203, USNM Genitalia Slide No. 12622. [Elachistidae: Stenomatinae]

fregeis Hodges, 1978 (Anoncia); Moths of America north of Mexico, fasc. 6.1: 67. Holotype + , California, Kern Co., Carrizo Plains, 20 August 1935, G. D. Hanna. USNM Genitalia Slide No. 6129. [Cosmopterigidae: Cosmopteriginae]

fritillella Powell, 1973 (Ethmia); Smithsonian Contributions to Zoology 120: 136. Holo-

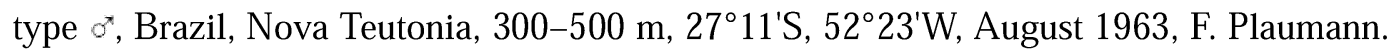
USNM Genitalia Slide No. 87689. [Elachistidae: Ethmiinae]

frondifer Busck, 1914 (Stenoma); Proceedings of the U.S. National Museum 47: 42. Holotype ${ }^{\star}$, Panama, Trinidad River, March 1912, A. Busck. USNM Type No. 16717, USNM Genitalia Slide No. 13824. [Elachistidae: Stenomatinae] 
fulmenella Busck, 1910 (Gelechia); Proceedings of the Entomological Society of Wash510 ington 11: 178. Holotype ${ }^{*}$, USA, California, Argus Mountains, April, A. Koebele. USNM Type No. 12697, Genitalia Slide AB Feb. 18, 1933. Left fore- and hindwing lacking. [Gelechiidae: Gelechiinae]

fulva Walsingham, 1882 (Depressaria); Transactions of the American Entomological Society 10: 175. Holotype $o^{\star}$, North America [USA], [no date], Fernald collection. [Elachistidae: Depressariinae]

fumipennis Busck, 1914 (Stenoma); Proceedings of the U.S. National Museum 47: 45. Holotype o*, Panama, Trinidad River, March 1912, A. Busck. USNM Type No. 16725, USNM Genitalia Slide No. 12888. [Elachistidae: Stenomatinae]

furia Hodges, 1986 (Dichomeris); Moths of America north of Mexico, fasc. 7.1: 93. Holotype ${ }^{\rtimes}$, USA, Illinois, Putnam Co., 11 July 1968, M. O. Glenn. [Gelechiidae: Dichomeridinae]

fusca Duckworth, 1964 (Lethata); Proceedings of the U.S. National Museum 116: 103. Holotype $\sigma^{\star}$, Brazil, Santarém, July 1927, Meyrick Collection, BM 1938-290. USNM Genitalia Slide No. 13387. [Elachistidae: Stenomatinae]

fusca Park \& Hodges, 1995 (Dichomeris); Insecta Koreana 12: 16. Holotype ${ }^{\star}$, Taiwan, Taichung Co., Hassenzan, 4 June 1942, S. Issiki. USNM Genitalia Slide No. 87474. [Gelechiidae: Dichomeridinae]

fuscalis Park \& Hodges, 1995 (Dichomeris); Insecta Koreana 12: 16. Holotype o*, Taiwan, Taipei Co., Taihoku, 7 June 1946, S. Issiki. USNM Genitalia Slide No. 11683. [Gelechiidae: Dichomeridinae]

fuscipalpalis Becker, 1982 (Timocratica); Bulletin of the British Museum (Natural History), Entomology 45: 246. Holotype ${ }^{*}$, Venezuela, Guayaraca, Auyantepui, Bolivar, 1100 m, 14 April 1956, F. Fernandez \& C. J. Rosales. W. D. Duckworth Genitalia Slide No. 3694. [Elachistidae: Stenomatinae]

fusciterminella Clarke, 1941 (Agonopterix); Proceedings of the U.S. National Museum 90: 80. Holotype $\sigma^{\star}$, Canada, British Columbia, Duncan, Vancouver Island, 13 July 1910, Hanham. USNM Type No. 52077, USNM Genitalia Slide No. 125. [Elachistidae: Depressariinae] 
fuscolepis Park, 2002 (Nosphistica), Zoological Studies 41: 257. Holotype o*, Taiwan, Kaohsiung Co., 1-2 km W Meishan, 29 June-2 July 1980, D. Davis. Specimen not found. [Lecithoceridae]

fuscorectangulata Duckworth, 1964 (Antaeotricha); Proceedings of the U.S. National Museum 116: 41. Holotype $\sigma^{*}$ USA, Arizona, Chiricahua Mts., South Fork of Cave Creek, 4 July 1939, A. F. Braun. W. D. Duckworth Genitalia Slide No. 2002. [Elachistidae: Stenomatinae]

fuscotaeniaella Chambers, 1878 (Gelechia); Bulletin of the U.S. Geological and Geographic Survey of the Territories 4: 89. Pseudotype ${ }^{*}$, USA, Texas [no date]. USNM Type No. 451. According to Miller and Hodges, the holotype is in MCZ. [Gelechiidae: Gelechiinae]

gaiophanes Clarke, 1971 (Gonionota); Smithsonian Contributions to Zoology 95: 13. Holotype 9 , Brazil, New Bremen, 7 February 1936, F. Plaumann. USNM Type No. 71091, USNM Genitalia Slide No. 68761. [Oecophoridae: Oecophorinae]

gallaesolidaginis Riley, 1869 (Gelechia); First Annual Report on the Noxious, Beneficial and Other Insects of the State of Missouri: 173. Holotype ${ }^{\star}$, USA, Illinois, Chicago, em. 18 August 1867. USNM Genitalia Slide No. 6985. [Gelechiidae: Gelechiinae]

gallaespeciosum Miller, 2000 (Gnorimoschema); Thomas Say Publications in Entomology: 38. Holotype ${ }^{\star}$, Minnesota, Ramsey Co., Roseville, r.f. Solidago speciosa, 1972, W. E. Miller. USNM Genitalia Slide No. 90896. [Gelechiidae: Gelechiinae]

gallegoi Clarke, 1968 (Maesara); Proceedings of the U.S. National Museum 125: 2. Holotype ${ }^{*}$, Colombia, Antioquia, Rio Negro, October 1966. USNM Type No. 69716, USNM Genitalia Slide No. 69527. [Oecophoridae: Oecophorinae]

gargantuella Heinrich, 1920 (Holcocera); Proceedings of the U.S. National Museum 57: 69. Holotype ${ }^{*}$, USA, Arizona, Brush Corral, r.f. galls on white oak, em. 7-15 May, W. D. Edmonston \& G. Hofer. USNM Type No. 21810, Genitalia Slide CH, 13 December 1917. [Coleophoridae: Blastobasinae]

garritor Hodges, 1966 (Batrachedra); Transactions of the American Entomological Society 92: 611. Holotype + , USA, Arizona, Coconino Co., Hochderffer Hill, 12.5 mi NNW Flagstaff, 8500', 9 July 1961, R. W. Hodges. USNM Type No. 67859, USNM Genitalia Slide No. 5094. [Batrachedridae] 
garveyi Adamski \& Brown, 2001 (Glyphidocera); Proceedings of the Entomological Society of Washington 103: 989. Holotype o*, Venezuela, Territorio Federal Amazona, Cerro de la Neblina, base camp, $140 \mathrm{~m}, 0^{\circ} 50^{\prime} \mathrm{N}, 66^{\circ} 9^{\prime} \mathrm{W}, 24$ November-1 December 1984, R. Brown. USNM Genitalia Slide No. 81458. [Glyphidoceridae]

gelidella Busck, 1908 (Depressaria); Proceedings of the Entomological Society of Washington 9: 90. Holotype ${ }^{*}$, Canada, Manitoba, Winnipeg, [no date], A. W. Hanham. USNM Type No. 11328. Abdomen lacking. [Oecophoridae: Depressariinae]

geminata Clarke, 1978 (Aliciana); Smithsonian Contributions to Zoology 273: 65. Holotype ${ }^{*}$, Chile, Malleco, Rio Blanco, 1050-1300 m, 21-24 February 1954, L. E. Peña. USNM Type No. 73726, USNM Genitalia Slide No. 24259. [Oecophoridae: Oecophorinae]

geniata Hodges, 1966 (Lita); Proceedings of the U.S. National Museum 119: 18. Holotype or, USA, California, San Diego Co., La Puerta Valley [no date]. [Gelechiidae: Gelechiinae]

geniatella Busck, 1914 (Gelechia); Proceedings of the U.S. National Museum 47: 13. Holotype $\&$, Panama, Corozal, Isthmian Canal Zone, March 1911, A. Busck. USNM Type No. 15827, USNM Genitalia Slide No. 10662. [Gelechiidae: Gelechiinae]

georgei Hodges, 1985 (Tildenia); Journal of the Lepidopterists' Society 39: 151. Holotype ๙, USA, Illinois, Mason Co., Sand Ridge State Forest, 16 August 1982, r.f. Physalis heterophylla var. ambigua, em. 7 September 1982, P. Gross. [Gelechiidae: Gelechiinae]

geranella Barnes \& Busck, 1920 (Ethmia); Contributions to the Natural History of the Lepidoptera of North America 4: 242. Holotype ox, USA, California, San Diego Co., La Puerta Valley [no date]. USNM Type No. 22673. [Elachistidae: Ethmiinae]

gerda Busck, 1914 (Gonioterma); Proceedings of the U.S. National Museum 47: 52. Holotype $\sigma^{\star}$, Panama, Porto Bello, April 1912, A. Busck. USNM Type No. 16740, USNM Genitalia Slide No. 14127. [Elachistidae: Stenomatinae]

gibsoniella Busck, 1915 (Gnorimoschema); Proceedings of the Entomological Society of Washington 17: 82. Holotype + , Canada, Manitoba, Aweme, r.f. Solidago rigida, em. 11 August 1914, N. Criddle. USNM Type No. 19227, USNM Genitalia Slide No. 7011. [Gelechiidae: Gelechiinae] 
gigantea Busck, 1914 (Ethmia); Insecutor Inscitiae Menstruus 2: 54. Holotype ${ }^{\circ}$, Mexico,

gioia Clarke, 1951 (Coptotelia); Acta Zoologica Lilloana 11: 347. Holotype + , Bolivia, Incachaca. Cochabamba, [no date], J. Steinbach. USNM Type No. 61115, USNM Genitalia Slide No. 69426. [Oecophoridae: Oecophorinae]

glandulella Riley, 1871 (Gelechia); Canadian Entomologist 3: 118. Lectotype + , USA, Missouri. USNM Type No. 471, USNM Genitalia Slide No. 81479. Designated by Adamski \& Brown (1989). [Coleophoridae: Blastobasinae]

gleba Hodges, 1986 (Dichomeris); Moths of America north of Mexico, fasc. 7.1: 87. Holotype 9 , USA, Illinois, Putnam Co., 31 July 1972, M. O. Glenn. [Gelechiidae: Dichomeridinae]

glebula Clarke, 1978 (Doina); Smithsonian Contributions to Zoology 273: 33. Holotype \%, Chile, Santiago, Rio Colorado, 10 April 1953, L. E. Peña. USNM Type no. 73710, USNM Genitalia Slide No. 24265. [Amphisbatidae]

gleditschiaeella Chambers, 1876 (Laverna?); Canadian Entomologist 8: 136. Holotype $0^{x}$, USA, Kentucky, r.f. thorns of Gleditschia sp. [no date]. [Gelechiidae: Chrysopeleiinae]

glenni Clarke, 1947 (Dichomeris); Proceedings of the Entomological Society of Washington 49: 187. Holotype ${ }^{*}$, USA, Illinois, Putnam Co., 5 August 1946, M. O. Glenn. USNM Type No. 58309, USNM Genitalia Slide No. 24265. [Gelechiidae: Dichomeridinae]

glenni Kaila, 1996 (Elachista); Entomologica Scandinavica 27: 234. Holotype ơ, USA, Illinois, Putnam Co., 7 July 1965, M. O. Glenn. USNM Genitalia Slide No. 89284. [Elachistidae: Elachistinae]

glowackae Adamski \& Brown, 2001 (Glyphidocera); Proceedings of the Entomological Society of Washington 103: 972. Holotype ox, Venezuela, Territorio Federal Amazona, Cerro de la Neblina, camp VII, 051'N, 6558'W, 1850 m, 2-4 December 1984, R. Brown. USNM Genitalia Slide No. 81200. [Glyphidoceridae]

gnoma Hodges, 1978 (Melanocinclis); Moths of America north of Mexico, fasc. 6.1: 43. Holotype $\diamond^{*}$, USA, Florida, Archbold Biological Station, Lake Placid, 8-15 May 1964, R. W. Hodges. USNM Type No. 70563, USNM Genitalia Slide No. 4682. [Cosmopterigidae: Cosmopteriginae] 
golmeia Hodges, 1974 (Carolana); Moths of America north of Mexico, fasc. 6.2: 126. 510 Holotype + , USA, Arizona, Coconino Co., $16 \mathrm{mi}$ SW Flagstaff, West Fork, 6500', 20 August 1961, R. W. Hodges. USNM Genitalia Slide No. 1092. [Oecophoridae: Oecophorinae]

golovina Clarke, 1947 (Filatima); Journal of the Washington Academy of Sciences 37: 264. Holotype ${ }^{\star}$, USA, California, Riverside Co., Palm Springs, 2 May 1921, K. R. Coolidge. Specimen not found. [Gelechiidae: Gelechiinae]

goodellella Chambers, 1881 (Gelechia); Journal of the Cincinnati Society of Natural History 3: 289. Holotype (uncertain sex), USA, Massachusetts, Amherst [no date]. [Gelechiidae: Dichomeridinae]

grabia Wu \& Park, 1999 (Homaloxestis); Insecta Koreana 16: 1. Holotype ${ }^{\star}$, Sri Lanka, Kan. District, Udawattakele, 1800', 19 November 1976, black light, Hevel, Dietz, Karunaratne \& Balasooriya. USNM Genitalia Slide No. 89023. [Lecithoceridae]

gracilens Hodges, 1962 (Cosmopterix); Entomologica Americana (n.s.) 42: 30. Holotype $\%$, USA, Arizona, Huachuca Mountains, Palmerlee [no date]. USNM Type No. 66079, USNM Genitalia Slide No. 1349. [Cosmopterigidae: Cosmopteriginae]

graminea Adamski, 1999 (Blastobasis); Proceedings of the Entomological Society of Washington 101: 165. Holotype $q$, Colombia, Cauca Valley, Instituto Colombiano Agropecuario, Experiment Station Palmira, ex-sugar cane, 1-15 March 1991, em. 21 April-1 May 1991, L. Cardenas Duque. [Coleophoridae: Blastobasinae]

grandis Clarke, 1947 (Chionodes); Journal of the Washington Academy of Sciences 37: 253. Holotype $\sigma^{*}$, USA, Colorado, Silverton, 8-15 July. USNM Type No. 58242, USNM Genitalia Slide No. 7901. [Gelechiidae: Gelechiinae]

grandis Hodges, 1978 (Perimede); Moths of America north of Mexico, fasc. 6.1: 130. Holotype $\sigma^{*}$, USA, Texas, Culberson Co., Guadalupe Mountains, McKittrick Canyon, 5000', 23 May 1973, R. W. Hodges. USNM Genitalia Slide No. 4723. [Cosmopterigidae: Chrysopeleiinae]

grandis Koster, 2002 (Chrysoclista); Tijdschrift voor Entomologie 145: 107. Holotype $\odot^{\star}$, USA, Colorado, Chaffee Co., 2 mi ESE Buena Vista, 3849'49"N, 10605'10"W, 8430', 23 August 1997, D. J. Wright. USNM Genitalia Slide No. 82491. [Elachistidae: Agonoxeninae] 
granosa Kaila, 1997 (Elachista); Acta Zoologica Fennica 206: 36. Holotype o ${ }^{\star}$, USA, Arizona, Coconino Co., 9.5 mi SE Flagstaff, Vail Lake Road, 6500', 18 July 1961, R. W. Hodges. USNM Genitalia Slide No. 89282. [Elachistidae: Elachistinae]

graphica Busck, 1920 (Stenoma); Insecutor Inscitiae Menstruus 8: 91. Holotype ơ, Costa Rica, Sixola River, April 1907, W. Schaus. USNM Type No. 22312, USNM Genitalia Slide No. 13826. [Elachistidae: Stenomatinae]

graphicella Busck, 1908 (Gnorimoschema); Proceedings of the Entomological Society of Washington 9: 86. Holotype ${ }^{*}$, USA, California [no date]. USNM Type No. 11321, USNM Genitalia Slide No. 6257. [Gelechiidae: Gelechiinae]

gratus Hodges, 1999 (Chionodes); Moths of America north of Mexico, fasc. 7.6: 203. Holotype + , USA, Montana, Sweetgrass Co., Crazy Mountains, Half Moon Park, Big Timber Canyon, 6500', 26 June 1966, D. C. Ferguson. USNM Genitalia Slide No. 472. [Gelechiidae: Gelechiinae]

grissefasciella Chambers, 1875 (Gelechia); Cincinnati Quarterly Journal of Science 2: 253. Holotype $\sigma^{*}$ USA, Texas, [no date]. Right forewing and abdomen lacking. [Gelechiidae: Gelechiinae]

grypotatos Park, 2001 (Carodista), in Park \& Wu, Insecta Koreana 18: 145. Holotype o*, Sri Lanka, Gal. District, Kanneliya Jungle, 11-16 January 1975, S. Wood \& J. Petty. Specimen not found. [Lecithoceridae]

guilandinae Busck, 1900 (Blastobasis); Proceedings of the U.S. National Museum 23: 234. Lectotype $\sigma^{\star}$, USA, Florida, Palm Beach, r.f. stem of Guilandinae bonducella, em. 30 March 1900. USNM Type No. 4942, USNM Genitalia Slide No. 80980. Designated Adamski \& Hodges (1996). [Coleophoridae: Blastobasinae]

gunni Busck, 1911 (Stenoma); Proceedings of the U.S. National Museum 40: 218. Holotype ${ }^{\star}$, French Guiana, St. Laurent, Maroni River, [no date], W. Schaus. USNM Type No. 13577, USNM Genitalia Slide No. 17759. [Elachistidae: Stenomatinae]

guttata Busck, 1914 (Eritarbes); Proceedings of the U.S. National Museum 47: 1. Holotype ${ }^{\star}$, Panama, Cabima, June, A. Busck. USNM Type No. 16668. [Cosmopterigidae: Chrysopeleiinae]

habra Hodges, 1978 (Teladoma); Moths of America north of Mexico, fasc. 6.1: 51. Holotype ${ }^{*}$, USA, Arkansas, Washington Co., Devil's Den State Park, 22 May 1966, R. W. 
habrochitona Walsingham, 1911 (Dichomeris); Biologia Centrali-Americana, Lepidoptera Heterocera 4: 102. Holotype ox, Panama, Canal Zone, Tabernilla, June 1907, A. Busck. [Gelechiidae: Dichomeridinae]

hagenella Chambers, 1878 (Anesychia); Bulletin of the U.S. Geological and Geographic Survey of the Territories 4: 80. Pseudotype ${ }^{\star}$, USA, Texas, "27." USNM Type No. 436, USNM Genitalia Slide No. 89951. Powell (1973) designated a lectotype in MCZ. [Elachistidae: Ethmiinae]

hageter Hodges, 1966 (Batrachedra); Transactions of the American Entomological Society 92: 616. Holotype ${ }^{\star}$, USA, California, Mt. Lowe, 28 July 1924. USNM Type No. 67862, USNM Genitalia Slide No. 5111. Right fore- and hindwing lacking. [Batrachedridae]

haldarella Kaila, 1999 (Elachista); Acta Zoologica Fennica 211: 20. Holotype ox, USA, Nevada, Lander Co., Toiyabe National Forest, Bob Scott Campground, 1 June 1974, 7300-7500', J. F. G. Clarke. USNM Genitalia Slide No. 90099. [Elachistidae: Elachistinae]

hallwachsae Clarke, 1983 (Eomichla); Journal of the Lepidopterists' Society 37: 155. Holotype ${ }^{*}$, Costa Rica, Guanacaste Province, Santa Rosa National Park, r.f. Bombacopsis quinatum, 23-25 May 1980, D. Janzen \& W. Hallwachs. [Oecophoridae: Oecophorinae]

hamatella Adamski \& Brown, 2001 (Glyphidocera); Proceedings of the Entomological Society of Washington 103: 979. Holotype ${ }^{*}$, Venezuela, Territorio Federal Amazona, Cerro de la Neblina, base camp, $140 \mathrm{~m}, 0^{\circ} 50^{\prime} \mathrm{N}, 66^{\circ} 9^{\prime} \mathrm{W}, 4-12$ February 1984, D. Davis \& T. McCabe. USNM Genitalia Slide No. 81632. [Glyphidoceridae]

hammella Busck, 1910 (Ethmia); Proceedings of the Entomological Society of Washington 12: 53. Holotype + , Costa Rica, Tuis, 5800', [no date], W. Schaus. USNM Type No. 12844. [Elachistidae: Ethmiinae]

hamon Busck, 1911 (Stenoma); Proceedings of the U.S. National Museum 40: 223. Holotype $q$, French Guiana, St. Jean, Maroni River, [no date], W. Schaus. USNM Type No. 13589, USNM Genitalia Slide No. 12980. [Elachistidae: Stenomatinae]

hapsus Hodges, 1999 (Chionodes); Moths of America north of Mexico, fasc. 7.6: 55. Holotype ơ, USA, Arkansas, Washington, Co., Devil's Den State Park, 21 July 1966, R. W. Hodges. [Gelechiidae: Gelechiinae] 
harrisonella Busck, 1904 (Aristotelia); Proceedings of the U.S. National Museum 27: 756. Holotype 9 , Canada, British Columbia, Kalso, July, Dyar \& Cockle. USNM Type No. 7855, USNM Genitalia Slide No. 5878. [Gelechiidae: Gelechiinae]

hassenzanum Park \& Hodges, 1995 (Helcystogramma); Korean Journal of Systematic Zoology 11: 229. Holotype o*, Taiwan, Taichung Co., Hassenzan, 4 June 1947, S. Issiki. USNM Genitalia Slide No. 11680. [Gelechiidae: Dichomeridinae]

haywardi Busck, 1938 (Timocratica); Anales de la Sociedad Cientifica de Argentina 126: 280. Holotype (uncertain sex), Argentina [no date]. Specimen not found. [Elachistidae: Stenomatinae]

hebes Dognin, 1905 (Mesoptycha); Annales de la Société Entomologique de Belgique 49: 89. Holotype ${ }^{*}$, Colombia, Micay, Août 1896, Durrant “12.6.12," Dognin Collection. USNM Type No. 32366, USNM Genitalia Slide No. 14048. [Elachistidae: Stenomatinae]

heidemannella Dietz, 1900 (Epigritia); Transactions of the American Entomological Society 36: 111. Lectotype $\sigma^{\star}$, USA, Pennsylvania, Bedford Co., Sulphur Springs, 1900. USNM Type No. 6133, USNM Genitalia Slide No. 81053. Specimen not found. Designated by Adamski \& Hodges (1996). [Coleophoridae: Blastobasinae]

helianthi Busck, 1932 (Teladoma); Proceedings of the Entomological Society of Washington 34: 17. Lectotype $\sigma^{\star}$, USA, Illinois, St. Louis, r.f. sunflower. USNM Type No. 43657. Right fore-and hindwing lacking. Designated by Clarke (1951). [Cosmopterigidae: Cosmopteriginae]

hemiglypta Clarke, 1971 (Gonionota); Smithsonian Contributions to Zoology 95: 15. Holotype $\sigma^{\star}$, Brazil, Nova Teutonia, April 1948, F. Plaumann. USNM Type No. 71092, USNM Genitalia Slide No. 68778. [Oecophoridae: Oecophorinae]

hemileucus Clarke, 1986 (Asymphorodes); Smithsonian Contributions to Zoology 416: 205. Holotype ${ }^{\prime}$, Marquesas Archipelago, Nuku Hiva, Tapuaooa, 2500', 30 January 1968, J. F. G. \& T. M. Clarke. USNM Type No. 100784, USNM Genitalia Slide No. 24729. [Cosmopterigidae: Cosmopteriginae]

hemilitha Clarke, 1965 (Gnorimoschema); Proceedings of the U.S. National Museum 117: 81. Holotype $\star^{\star}$, Chile, Juan Fernandez Islands, Masatierra, Bahia Cumberland, 17 February 1951. [Gelechiidae: Gelechiinae] 
hemisigna Clarke, 1951 (Parastega); Journal of the Washington Academy of Sciences 41: 140. Holotype $\sigma^{*}$, Argentina, Buenos Aires, June 1950, F. Bourquin. USNM Type No. 60941, USNM Genitalia Slide No. 13006. Left fore- and hindwing lacking. [Gelechiidae: Gelechiinae]

henicosura Wu \& Park, 1999 (Doxogenes); Korean Journal of Systematic Zoology 15: 215. Holotype o*, Sri Lanka, Gal. District, Kanneliya, 200', 15-17 October 1976, Hevel, Dietz, Karunaratne \& Balasooriya. USNM Genitalia Slide No. 89158. Specimen not found. [Lecithoceridae]

henshawiella Busck, 1903 (Gnorimoschema); Proceedings of the U.S. National Museum 25: 831. Holotype 9 , USA, Colorado [no date]. USNM Type No. 6362, USNM Genitalia Slide No. 7243. [Gelechiidae: Gelechiinae]

heptathalama Busck, 1900 (Homaledra); Proceedings of the U.S. National Museum 23: 237. Syntypes $\left(10^{\star}, 1\right.$ ㅇ), USA, Florida, Palm Beach, r.f. Sabal palmetto, [no date], H. Dyar \& F. Kinzel. USNM Type No. 4946, USNM Genitalia Slide No. 4893, USNM Wing Slide No. 4891. Described from 8 specimens; we were unable to find the other 6 . [Coleophoridae: Coleophorinae]

hermosella Busck, 1911 (Filinota); Proceedings of the U.S. National Museum 40: 207. Holotype $\sigma^{x}$, French Guiana, St. Jean, Maroni River, [no date], W. Schaus. USNM Type No. 13559, USNM Genitalia Slide No. 69325. Right fore- and hindwing lacking. [Oecophoridae: Oecophorinae]

hesitans Diakonoff, 1968 (Chrysonoma); Bulletin of the U.S. National Museum 257 (1967): 180. Holotype ${ }^{*}$, Philippine Islands, Luzon, Mt. Apo, 70-B, 5 March 1945. USNM Genitalia Slide No. 89983. [Oecophoridae: Oecophorinae]

hesphoea Hodges, 1975 (Agonopterix); Journal of the Lepidopterists' Society 29: 89. Holotype $\sigma^{*}$, USA, Texas, Culberson Co., Sierra Diablo, 20 mi NNW Van Horn, 6000', 27 May 1973, R. W. Hodges. USNM Genitalia Slide No. 4653, USNM Type No. 73061. Right hindleg in gelatin vial. [Elachistidae: Depressariinae]

heterochroma Clarke, 1971 (Hypercallia); Smithsonian Contributions to Zoology 95: 36. Holotype $\sigma^{*}$, Venezuela, Aragua, Rancho Grande, 22-31 July 1967, R. W. Poole. USNM Type No. 71103, USNM Genitalia Slide No. 69434. [Oecophoridae: Oecophorinae]

heterotoma Diakonoff, 1968 (Brachmia); Bulletin of the U.S. National Museum 257 (1967): 158. Holotype ơ, Philippine Islands, Luzon, Los Baños, [no date], P. J. Baker. USNM Genitalia Slide No. 89979. [Gelechiidae: Gelechiinae] 
hibiscella Busck, 1903 (Gelechia); Proceedings of the U.S. National Museum 25: 869. Lectotype + , USA, District of Columbia, r.f. Hibiscus moscheutos, “3476 11/8 84," C. V. Riley. Designated by Hodges (1999). USNM Type No. 6383, USNM Genitalia Slide No. 7905. Right fore- and hindwing lacking. [Gelechiidae: Gelechiinae]

hieroglyphica Powell, 1973 (Ethmia); Smithsonian Contributions to Zoology 120: 185. Holotype $\sigma^{x}$, Bolivia, Incachaca, Cochabamba, [no date], J. Steinbach. USNM Genitalia Slide No. 98456. [Elachistidae: Ethmiinae]

hiramella Busck, 1914 (Ethmia); Insecutor Inscitiae Menstruus 2: 56. Holotype ${ }^{\star}$, Cuba, Santiago, [no date], W. Robson. USNM Type No. 18272, USNM Genitalia Slide No. 87684. According to the original description, the specimen was collected by W. Schaus. [Elachistidae: Ethmiinae]

hirculella Busck, 1909 (Dichomeris); Proceedings of the Entomological Society of Washington 11: 89. Lectotype + , USA, Connecticut, East River, 18 July 1908, C. Ely. Designated by Hodges (1986). USNM Type No. 12264, USNM Genitalia Slide No. 10684. [Gelechiidae: Dichomeridinae]

hodgesi Povolný, 1967 ("Ephysteris"); Acta Entomologica Musei Nationalis Pragae 37: 119. Holotype + , USA, Arizona, Coconino Co., Fort Valley, $7.5 \mathrm{mi}$ NW Flagstaff, 12 July 1961, R. W. Hodges. USNM Genitalia Slide No. 4634. [Gelechiidae: Gelechiinae]

homosoma Clarke, 1986 (Asymphorodes); Smithsonian Contributions to Zoology 416: 229. Holotype $\sigma^{\star}$, Marquesas Archipelago, Nuku Hiva, Taiohae, 4 February 1968, J. F. G. \& T. M. Clarke. USNM Type No. 100792, USNM Genitalia Slide No. 24739. [Cosmopterigidae: Cosmopteriginae]

honoria Clarke, 1986 (Asymphorodes); Smithsonian Contributions to Zoology 416: 272. Holotype ${ }^{*}$, Marquesas Archipelago, Fatu Hiva, Mt. Upe, 2025', 3 April 1968, J. F. G. \& T. M. Clarke. USNM Type No. 100816, USNM Genitalia Slide No. 24939. [Cosmopterigidae: Cosmopteriginae]

hora Busck, 1914 (Psoricoptera); Proceedings of the U.S. National Museum 47: 14. Holotype + , Panama, Alhajuela, April 1911, A. Busck. USNM Type No. 15828. [Gelechiidae: Gelechiinae]

hortatrix Hodges, 1969 (Periploca); Smithsonian Contributions to Zoology 18: 4. Holotype + , USA, Arkansas, Washington Co., Devil's Den State Park, 20 July 1966, R. W. Hodges. USNM Type No. 69779, USNM Genitalia Slide No. 4288. [Cosmopterigidae: Chrysopeleiinae] 

$o^{\star}$, USA, Florida, Pensacola, 13 May 1962, S. Hills. USNM Type No. 69535. [Amphisbatidae]

hostiata Hodges, 1969 (Periploca); Smithsonian Contributions to Zoology 18: 3. Holotype ๔, USA, Washington, Yakima Co., 2.5 mi W Fort Simcoe, 31 July 1962, J. F. G. Clarke. USNM Type No. 69791, USNM Genitalia Slide No. 4287. [Cosmopterigidae: Chrysopeleiinae]

hostis Hodges, 1999 (Chionodes); Moths of America north of Mexico, fasc. 7.6: 122. Holotype $\sigma^{\pi}$, USA, Utah, Sanpete Co., Major's Flat near Ephraim Canyon, 20 July 1981, R. W. Hodges. [Gelechiidae: Gelechiinae]

howardi Walsingham, 1909 (Aristotelia); Biologia Centrali-Americana, Lepidoptera Heterocera 4: 23. Holotype $\sigma^{\star}$, Mexico, Sonora, San José de Guaymas, 10 April, L. O. Howard. Genitalia Slide AB July 1, 1933. Described from unique male. [Gelechiidae: Gelechiinae]

huachucella Busck, 1908 (Cryptolechia); Proceedings of the U.S. National Museum 35: 195. Holotype $\sigma^{\star}$, USA, Arizona, Huachuca Mts. [no date]. USNM Type No. 11941, USNM Genitalia Slide No. 725. [Elachistidae: Depressariinae]

huffmanellum Metzler \& Adamski, 2002 (Gnorimoschema); Fabreries 27: 61. Holotype ${ }^{\star}$, USA, Ohio, Greene Co., Wright-Patterson Air Force Base, Huffman Prairie, site B, 3 June 1994, E. Metzler. [Gelechiidae: Gelechiinae]

hurlberti Adamski, 2000 (Glyphidocera); Fabreries 25: 70. Holotype ơ USA, Colorado, Chaffee Co., below Cottonwood Pass, 10,200', 16 July 1982, D. C. Ferguson. USNM Genitalia Slide No. 82256. [Glyphidoceridae]

hyperella Ely, 1910 (Agonopterys) (sic); Proceedings of the Entomological Society of Washington 12: 68. Lectotype $\sigma^{\star}$, USA, Virginia, Great Falls, 26 April 1909, r.f. Hypericum prolificum, em. 26-27 May 1909, C. R. Ely. USNM Type No. 12853, USNM Genitalia Slide No. 6. Designated by Hodges (1974). [Elachistidae: Depressariinae]

hypoleuca Clarke, 1971 (Gonionota); Smithsonian Contributions to Zoology 95: 10. Holotype $\sigma^{*}$, Venezuela, Aragua, Rancho Grande, 11-15 January 1966, S. S. \& W. D. Duckworth. USNM Type No. 71088, USNM Genitalia Slide No. 68753. [Oecophoridae: Oecophorinae] 
hypostema Clarke, 1986 (Asymphorodes); Smithsonian Contributions to Zoology 416: 201. Holotype $\sigma^{\star}$, Marquesas Archipelago, Nuku Hiva, Tunoa Ridge, 2900', 23 January 1968, J. F. G. \& T. M. Clarke. USNM Type No. 100782, USNM Genitalia Slide No. 24958. [Cosmopterigidae: Cosmopteriginae]

hyptiotes Clarke, 1964 (Gonionota); Proceedings of the U.S. National Museum 115: 80. Holotype + , Mexico, Orizaba, 10 September, R. Müller. USNM Type No. 66380, USNM Genitalia Slide No. 68974. [Oecophoridae: Oecophorinae]

iceryaeella Riley, 1887 (Blastobasis); Report of the Commissioner of Agriculture, 1886: 484. Lectotype $\sigma^{*}$, USA, California, Alameda Co. [no date]. USNM Type No. 473. Abdomen and right forewing lacking. Designated by Adamski \& Brown (1989). [Coleophoridae: Blastobasinae]

ignicolor Busck, 1914 (Cryptolechia); Proceedings of the U.S. National Museum 47: 27. Holotype ${ }^{\star}$, Panama, Cabima, May 1911, A. Busck. USNM Type No. 16746, USNM Genitalia Slide No. 4082. [Elachistidae: Depressariinae]

ignita Busck, 1912 (Lupercalia); Smithsonian Miscellaneous Collections 59: 7. Holotype ${ }^{\star}$, Panama, Trinidad River, May, A. Busck. USNM Type No. 14533, USNM Genitalia Slide No. 69328, USNM Wing Slide No. 69328. [Oecophoridae: Oecophorinae]

illuminella Busck, 1914 (Cryptolechia); Proceedings of the U.S. National Museum 47: 28. Holotype ${ }^{*}$, Panama, Trinidad River, March 1912, A. Busck. USNM Type No. 16747. Abdomen lacking. [Elachistidae: Depressariinae]

illusio Hodges, 1986 (Dichomeris); Moths of America north of Mexico, fasc. 7.1: 101. Holotype + , USA, Florida, Hastings, 8 June. USNM Genitalia Slide No. 12203. USNM Genitalia Slide No. 12203. [Gelechiidae: Dichomeridinae]

illusor Hodges, 1966 (Batrachedra); Transactions of the American Entomological Society 92: 604. Holotype + , USA, Illinois, Putnam Co., r.f. twigs of Salix spp., 26 June 1964, M. O. Glenn. USNM Type No. 67853, USNM Genitalia Slide No. 5057. [Batrachedridae]

imber Hodges, 1999 (Chionodes); Moths of America north of Mexico, fasc. 7.6: 71. Holotype $\diamond^{\star}$, USA, Nebraska, Cherry Co., Hackberry Lake, Valentine National Wildlife Refuge, 7 June 1983, R. W. Hodges. [Gelechiidae: Gelechiinae]

imperiella Busck, 1914 (Peleopoda); Proceedings of the U.S. National Museum 47: 26. Holotype $\&$, Panama, La Chorrera, May 1912, A. Busck. USNM Type No. 16687, USNM Genitalia Slide No. 69542. [Peleopodidae] 
imperiella Jäckh, 1978 (Scythris); Bolletino del Museo Civico di Storia Naturali 5: 7. Holotype ${ }^{*}$, Italy, Passo di Teglia, 1100 m, 30 June 1969, E. Jäckh. Genitalia preparation affixed to pin. [Xyloryctidae: Scythridinae]

impes Hodges, 1999 (Chionodes); Moths of America north of Mexico, fasc. 7.6: 227. Holotype $\sigma^{*}$, USA, Washington, Whitman Co., Kamiak Butte, r.f. Antennaria dimorpha, 18 May 1934, J. F. G. Clarke. USNM Genitalia Slide No. 15159. [Gelechiidae: Gelechiinae]

impresella Busck, 1914 (Gonioterma); Proceedings of the U.S. National Museum 47: 51. Holotype ${ }^{\star}$, Panama, Trinidad River, March 1912, A. Busck. USNM Type No. 16739, USNM Genitalia Slide No. 13845. [Elachistidae: Stenomatinae]

improvisa Diakonoff, 1968 (Lecithocera); Bulletin of the U.S. National Museum 257 (1967): 146. Holotype 9 , Philippine Islands, Mindanao, July-August 1917. On the type label the species name is misspelled "impromisa." Genitalia mounted on small glass slide affixed to pin. [Lecithoceridae]

impudica Walsingham, 1911 (Phthorimaea); Biologia Centrali-Americana, Lepidoptera Heterocera 4: 51. Holotype ${ }^{*}$, Panama, Canal Zone, Tabernilla, 1907, A. Busck. USNM Genitalia Slide No. 10671. [Gelechiidae: Gelechiinae]

inaequalis Busck, 1909 (Gelechia); Proceedings of the Entomological Society of Washington 11: 180. Holotype 9 , New Mexico, Mesilla, C. N. Ainslie. USNM Type No. 12000. Abdomen lacking. [Gelechiidae: Gelechiinae]

incana Hodges, 1962 (Teladoma); Entomologica Americana (n.s.) 42: 85. Holotype $\odot^{*}$, USA, Illinois, Putnam Co., 10 July 1959, M. O. Glenn. USNM Type No. 66084, USNM Genitalia Slide No. 3974. [Cosmopterigidae: Cosmopteriginae]

incana Hodges, 1969 (Pseudotelphusa); Proceedings of the Entomological Society of Washington 71: 202. Holotype $\sigma^{*}$, USA, New York, Adams, r.f. Pyrus, em. 1 April 1962. USNM Type No. 70577. [Gelechiidae: Gelechiinae]

incicur Hodges, 1966 (Lita); Proceedings of the U.S. National Museum 119: 17. Holotype o*, California, Tulare Co., Smokey Valley, 6300', 28 September 1946, C. Henne. USNM Type No. 67643, USNM Genitalia Slide No. 7458. [Gelechiidae: Gelechiinae]

inconspicua Clarke, 1978 (Doina); Smithsonian Contributions to Zoology 273: 24. Holotype $\sigma^{*}$, Chile, Arauco, Caramavida, 25-31 December 1953, L. E. Peña. USNM Type No. 73702, USNM Genitalia Slide No. 24254. [Amphisbatidae] 
incontigua Clarke, 1964 (Gonionota); Proceedings of the U.S. National Museum 115: 74. Holotype + , Venezuela, Caracas [no date]. USNM Type No. 66375, USNM Genitalia Slide No. 68987. [Oecophoridae: Oecophorinae]

indiscriminata Clarke, 1965 (Nanodacna); Proceedings of the U.S. National Museum 117: 93. Holotype 9 , Chile, Santiago, Juan Fernandez Islands, Masatierra, Piedra Agujereada, 12 March 1955, P. G. Kuschal. J. F. G. Clarke Genitalia Slide No. 10757. [Elachistidae: Agonoxeniinae]

inga Busck, 1911 (Gonioterma); Proceedings of the U.S. National Museum 40: 225. Holotype ${ }^{*}$, French Guiana, Cayenne, Maroni River, [no date], W. Schaus. USNM Type No. 13593, USNM Genitalia Slide No. 13340. [Elachistidae: Stenomatinae]

inornata Walsingham, 1882 (Semioscopis); Transactions of the American Entomological Society 10: 174. Syntype(s), North America. Walsingham (1882) indicates "In Prof. Fernald's and Mr Allen's collections." [Elachistidae: Depressariinae]

inquilinella Busck, 1910 (Gelechia); Canadian Entomologist 42: 168. Holotype ?, USA, New York, Karner, r.f. gall of cecidomyiid on willow, em. 24 March 1910, E. P. Felt. USNM Type No. 13119, USNM Genitalia Slide No. 9553. [Gelechiidae: Gelechinae]

inquisitor Hodges, 1966 (Chedra); Transactions of the American Entomological Society 92: 639. Holotype 9 , USA, Illinois, Putnam Co., 26 June 1962, M. O. Glenn. USNM Type No. 67871, USNM Genitalia Slide No. 5183. [Batrachedridae]

inscitum Busck, 1911 (Stenoma); Proceedings of the U.S. National Museum 40: 219. Holotype 9 , French Guiana, St. Laurent, Maroni River, [no date], W. Schaus. USNM Type No. 13581, USNM Genitalia Slide No. 14166. [Elachistidae: Stenomatinae]

insigna Wu \& Park, 1999 (Philharmonia); Tinea 16: 67. Holotype o*, Sri Lanka, Vav. District, Parayanalankulam Irrigation Canal, 100', 25 mi NW Medawachchiya, 20-25 March 1970, D. Davis \& B. Rowe. USNM Genitalia Slide No. 88867. [Lecithoceridae]

insignata Clarke, 1971 (Gonionota); Smithsonian Contributions to Zoology 95: 18. Holotype ${ }^{*}$, Ecuador, environs de Loja, “10-86," Dognin Collection. USNM Type No. 71094, USNM Genitalia Slide No. 68915. [Oecophoridae: Oecophorinae]

insulana Clarke, 1968 (Gonionota); Proceedings of the U.S. National Museum 125: 5. Holotype $\diamond^{\star}$, West Indies, Dominica, 0.5 mi E Pont Casse, 13 January 1965, J. F. G. \& T. M. Clarke. USNM Type No. 69717, J. F. G. Clarke Genitalia Slide No. 11681. [Oecophoridae: Oecophorinae] 

Holotype ${ }^{\star}$, USA, Arkansas, Washington Co., Devil's Den State Park, 11 July 1966, R. W. Hodges. USNM Genitalia Slide No. 4272. [Cosmopterigidae: Chrysopeleiinae]

invariabilis Kearfott, 1908 (Gelechia); Journal of the New York Entomological Society 16: 184. Holotype $\sigma^{*}$, USA, Utah, Stockton, 5-9 September 1906, T. Spalding. According to the original description, this species was described from 9 specimens, with the type in Kearfott's personal collection. Specimen not found. [Gelechiidae: Gelechiinae]

invictella Busck, 1908 (Recurvaria); Entomological News 19: 316. Holotype ơ, USA, California, San Diego, 14 April 1907. USNM Type No. 11915. Abdomen lacking. [Gelechiidae: Gelechiinae]

io Busck, 1911 (Stenoma); Proceedings of the U.S. National Museum 40: 213. Holotype + , French Guiana, St. Laurent, Maroni River [no date]. USNM Type No. 13568, USNM Genitalia Slide No. 13602. [Elachistidae: Stenomatinae]

iota Clarke, 1986 (Herlinda); Smithsonian Contributions to Zoology 416: 285. Holotype ${ }^{*}$, Marquesas Archipelago, Nuku Hiva, Taiohae, 28 January 1968, J. F. G. \& T. M. Clarke. USNM Type No. 100825. [Cosmopterigidae: Cosmopteriginae]

iothalles Forbes, 1939 (Trichotaphe); Journal of the New York Entomological Society 47: 159. Holotype $\sigma^{*}$, USA, New Jersey, New Brunswick, August 1932. USNM Genitalia Slide No. 87168. [Gelechiidae: Dichomeridinae]

ipomoeae Busck, 1900 (Cosmopteryx); Proceedings of the U.S. National Museum 23: 235. Holotype $\sigma^{\star}$, USA, Florida, Palm Beach, r.f. Ipomaea sp., [no date], H. Dyar. USNM Type No. 4943, USNM Genitalia Slide No. 3946. [Cosmopterigidae: Cosmopteriginae]

irene Barnes \& Busck, 1920 (Stenoma); Contributions to the Natural History of the Lepidoptera of North America 4: 239. Holotype $\sigma^{*}$, USA, Texas, Brownsville [no date]. USNM Genitalia Slide No. 95108. [Elachistidae: Stenomatinae]

irenella Busck, 1911 (Peleopoda); Proceedings of the U.S. National Museum 40: 209. Holotype 9 , French Guiana, St. Jean, Maroni River [no date], W. Schaus. USNM Type No. 13563, USNM Genitalia Slide No. 69543. [Peleopodidae]

iridella Busck, 1907 (Mompha); Proceedings of the Entomological Society of Washington. 8: 96. Holotype (uncertain sex), USA, California, Claremont, [no date], C. F. Baker. USNM Type No. 9902. Head, prothorax, metathorax, and abdomen lacking. [Cosmopterigidae: Cosmopteriginae] 
iridescens Clarke, 1947 (Chionodes); Journal of the Washington Academy of Sciences 37: 244. Holotype ${ }^{*}$, USA, Washington, Pierce Co., American Lake, r.f. Arctostaphylos uva-

ursi, em. 5 June 1946, J. F. G. Clarke. USNM Type No. 58235, USNM Genitalia Slide No. 7705. [Gelechiidae: Gelechiinae]

iridostoma Diakonoff, 1968 (Metathrinca); Bulletin of the U.S. National Museum 257 (1967): 117. Holotype ${ }^{\star}$, Philippine Islands, Luzon, Mt. Makiling, [no date], P. J. Baker. USNM Gentialia Slide No. 89987. [Xyloryctidae: Xyloryctinae]

irreptor Hodges, 1999 (Chionodes); Moths of America north of Mexico, fasc. 7.6: 143. Holotype $\sigma^{\star}$, USA, Texas, Uvalde Co., Garner State Park, 6 October 1984, E. C. Knudson. USNM Genitalia Slide No. 14884. [Gelechiidae: Gelechiinae]

isa Clarke, 1947 (Martyrhilda); Journal of the Washington Academy of Sciences 37: 5. Holotype ${ }^{*}$, Canada, Manitoba, Aweme, 31 March 1904, N. Criddle. USNM Type No. 58014, USNM Genitalia Slide No. 979. [Elachistidae: Depressariinae]

isa Hodges, 1986 (Dichomeris); Moths of America north of Mexico, fasc. 7.1: 103. Holotype $\sigma^{*}$, USA, Oklahoma, Sequoyah Co., Tenkiller Lake, 3 mi W Blackgum, 6-9 July 1979, D. \& M. Davis. USNM Genitalia Slide No. 12214. [Gelechiidae: Dichomeridinae]

isaura Clarke, 1978 (Teresita); Smithsonian Contributions to Zoology 273: 64. Holotype *, Chile, Malleco, Rio Blanco, 21-24 February 1954, L. E. Peña. USNM Type No. 73725, USNM Genitalia Slide No. 24288, USNM Wing Slide No. 24224. [Oecophoridae: Oecophorinae]

ischnella Kaila, 1997 (Elachista); Acta Zoologica Fennica 206: 26. Holotype o*, USA, Texas, Culberson Co., Sierra Diablo, 20 mi NNW Van Horn, 6000', 27 May 1973, R. W. Hodges. USNM Genitalia Slide No. 89287. [Elachistidae: Elachistinae]

isotona Meyrick, 1932 (Antaeotricha); Exotic Microlepidoptera 4: 291. Holotype (uncertain sex), Panama, Trinidad River, March, A. Busck. Specimen not found. [Elachistidae: Stenomatinae]

issikii Clarke, 1962 (Agonopterix); Entomological News 73: 96. Holotype $\sigma^{*}$, Japan, Honsyû, Sinano, Tobira, r.f. spun leaves of Orixa japonica [no date]. USNM Type No. 65830, USNM Genitalia Slide No. 25937. [Elachistidae: Depressariinae]

ivae Busck, 1900 (Aristotelia); Proceedings of the U.S. National Museum 23: 225. Syntypes $\left(1 \sigma^{*}, 1^{\circ}\right)$, USA, Florida, Palm Beach, r.f. Iva frutscens, [no date], H. Dyar. USNM Type No. 4932, USNM Genitalia Slide No. 5884. [Gelechiidae: Gelechiinae] 
janae Adamski \& Brown, 2001 (Glyphidocera); Proceedings of the Entomological Society of Washington 103: 995. Holotype $q$, Venezuela, Territorio Federal Amazona, Cerro de la Neblina, base camp, $140 \mathrm{~m}, 0^{\circ} 50^{\prime} \mathrm{N}, 66^{\circ} 9^{\prime} \mathrm{W}, 4-12$ February 1984, D. Davis \& T. McCabe. USNM Genitalia Slide No. 81644. [Glyphidoceridae]

jocelynae Miller, 2000 (Gnorimoschema); Thomas Say Publications in Entomology: 23. Holotype ${ }^{*}$, USA, Washington, D.C., r.f. Solidago gigantea, 1955, W. E. Miller. USNM Genitalia Slide No. 90897. [Gelechiidae: Gelechiinae]

johnstoni Clarke, 1947 (Chionodes); Journal of the Washington Academy of Sciences 37: 246. Holotype ${ }^{\star}$, USA, California, Sonoma Co., Petaluma, 10 July 1935, E. C. Johnston. USNM Type No. 58286, USNM Genitalia Slide No. 7774. [Gelechiidae: Gelechiinae]

juliella Busck, 1908 (Depressaria); Proceedings of the Entomological Society of Washington 9: 91. Holotype $\sigma^{*}$, New Mexico, Pecos, [no date], T. D. A. Cockerell. USNM Type No. 11330. Abdomen lacking. [Elachistidae: Depressariinae]

juniperella Adamski, 1987 (Glyphidocera); Proceedings of the Entomological Society of Washington 89: 330. Holotype $\diamond^{*}$, USA, Mississippi, Monroe Co., Amfac Nursery, 25 August 1981, r.f. gold coast juniper, em. 14 September 1981, D. Tatum. [Glyphidoceridae]

juniperella Kearfott, 1903 (Recurvaria); Journal of the New York Entomological Society 11: 157. Holotype + , USA, New Jersey, Caldwell, 20 June 1905. USNM Type No. 6965, USNM Genitalia Slide No. 6246. [Gelechiidae: Gelechiinae]

juniperi Hodges, 1978 (Periploca); Moths of America north of Mexico, fasc. 6.1: 109. Holotype 9 , Wyoming, 6 mi NW Newcastle, 23 July 1965, em. 3 August 1965, r.f. Juniperus sp., R. W. Hodges. [Cosmopterigidae: Chrysopeleiinae]

kasyi Duckworth, 1966 (Falculina); Proceedings of the U.S. National Museum 118: 395. Holotype ${ }^{\star}$, Surinam, Moengo, Boven Cottica, 14 May 1927, Cornell Univ. Lot 760, Sub 55. USNM Type No. 67277, USNM Genitalia Slide No. 14143. Although there is no type label on the specimen, it is clearly the type. [Elachistidae: Stenomatinae]

kearfottella Barnes \& Busck, 1920 (Coleophora); Contributions to the Natural History of the Lepidoptera of North America 4: 244. Syntypes (3 ${ }^{+}$), USA, New Jersey, Essex Co., r.f. Salix sp., W. D. Kearfott. USNM Type No. 22674. Specimens not found. [Coleophoridae: Coleophorinae]

kearfottella Busck, 1903 (Aproaerema); Proceedings of the U.S. National Museum 25: 842. Holotype ${ }^{\star}$, USA, New Jersey, Essex Co. Park, 25 July, W. D. Kearfott. USNM Type No. 6370, USNM Genitalia Slide No. 10677. [Gelechiidae: Gelechiinae] 
kearfottella Busck, 1903 (Aristotelia); Proceedings of the U.S. National Museum 25: 803. Holotype $\sigma^{*}$, USA, New Jersey, Essex Co., July, W. D. Kearfott. USNM Type No. 6352, USNM Genitalia Slide No. 5967. [Gelechiidae: Gelechiinae]

kimballi Duckworth, 1964 (Mothonica); Proceedings of the U.S. National Museum 116: 47. Holotype ${ }^{\star}$, USA, Florida, Sarasota Co., Siesta Key, 24 January 1954, C. P. Kimball. J. F. G. Clarke Genitalia Slide No. 10324. Lacking fore- and hindwing. [Elachistidae: Stenomatinae]

kincaidella Busck, 1907 (Gelechia); Proceedings of the Entomological Society of Washington 8: 91. Holotype $\sigma^{*}$, USA, Wyoming, Rock Spring [no date]. USNM Type No. 9895. Abdomen lacking. [Gelechiidae: Gelechiinae]

kinzelella Busck, 1900 (Nealyda); Proceedings of the U.S. National Museum 23: 225. Holotype ${ }^{\star}$, USA, Florida, Palm Beach, r.f. Pisonia obtusata, [no date], H. Dyar. USNM Type No. 4936, USNM Genitalia Slide No. 5578. [Gelechiidae: Gelechiinae]

knabi Walsingham, 1909 (Homaledra); Biologia Centrali-Americana, Lepidoptera Heterocera 4: 9. Holotype 9 , Mexico, Vera Cruz, Cordoba, May, F. Knab, Type 5972, Wlsm det 1908. USNM Wing Slide No. 5125, USNM Genitalia Slide No. 5126. [Batrachedridae]

kubai Hodges, 1999 (Chionodes); Moths of America north of Mexico, fasc. 7.6: 188. Holotype o , USA, California, El Dorado Co., Pine Hill, 4 July 1979, em. 5 August 1979, B. Kuba. [Gelechiidae: Gelechiinae]

labes Hodges, 1969 (Periploca); Smithsonian Contributions to Zoology 18: 6. Holotype 9 , USA, Arizona, Coconino Co., Vail Lake Road, 9.5 mi SE Flagstaff, 6500', 11 July 1961, R. W. Hodges. USNM Type No. 69782, USNM Genitalia Slide No. 5767. [Cosmopterigidae: Chrysopeleiinae]

lacerta Wu \& Park, 1999 (Homaloxestis); Insecta Koreana 16: 2. Holotype o*, Sri Lanka, Kan. District, Udawattakele, 2100', 4-5 October 1976, Hevel, Dietz, Karunaratne \& Balasooriya. USNM Genitalia Slide No. 88887. [Lecithoceridae]

lactans Hodges, 1999 (Chionodes); Moths of America north of Mexico, fasc. 7.6: 74. Holotype ${ }^{\star}$, USA, Texas, Culberson Co., Sierra Diablo, 20 mi NNW Van Horn, 6000', 29 May 1973, R. W. Hodges. [Gelechiidae: Gelechiinae]

lactis Busck, 1911 (Stenoma); Proceedings of the U.S. National Museum 40: 216. Holotype ${ }^{\star}$, French Guiana, St. Jean, Maroni River, [no date], W. Schaus. USNM Type No. 13574, USNM Genitalia Slide No. 13123. [Elachistidae: Stenomatinae] 
ladonia Landry \& Wright, 1993 (Coleophora); Canadian Entomologist 125: 559. Holotype $0^{\star}$, USA, Florida, Gainesville, 26 August 1966, L. O’Berry. USNM Genitalia Slide No. 87225. [Coleophoridae: Coleophorinae]

laetifica Busck, 1920 (Stenoma); Insecutor Inscitiae Menstruus 8: 91. Holotype ?, Guatemala, Cayuga, [no date], W. Schaus. USNM Type No. 22313, USNM Genitalia Slide No. 13133. [Elachistidae: Stenomatinae]

laetitia Hodges, 1986 (Dichomeris); Moths of America north of Mexico, fasc. 7.1: 88. Holotype $\diamond^{\star}$, USA, Illinois, Putnam Co., 7 June 1962, M. O. Glenn. [Gelechiidae: Dichomeridinae]

lagneia Clarke, 1978 (Doina); Smithsonian Contributions to Zoology 273: 21. Holotype o* Chile, Lautara, November 1895, V. Izquerdo. USNM Type No. 73699. [Amphisbatidae]

laguna Busck, 1912 (Gnorimoschema); First Annual Report of the Laguna Marine Laboratory (Pomona College) 1912: 167. Holotype $\sigma^{*}$, USA, California, Los Angeles Co., Laguna Beach, [no date], C. F. Baker. USNM Type No. 14335, USNM Genitalia Slide No. 8127. [Gelechiidae: Gelechiinae]

lagunculariella Busck, 1900 (Anacampsis); Proceedings of the U.S. National Museum 23: 230. Holotype $\diamond^{x}$, USA, Florida, Palm Beach, r.f. Laguncularia racemosa, [no date], H. Dyar. [Gelechiidae: Gelechiinae]

lamellospina Wu \& Park, 1998 (Deltoplastis); Insecta Koreana 15: 4. Holotype ơ, Sri Lanka, Rat. District, Sinharaja Jungle, 2 mi S of Weddagala, 8-12 February 1977, blacklight trap, Krombein, Fernando, Balasooriya \& Gunawardane. USNM Genitalia Slide No. 89226. [Lecithoceridae]

laminospina Wu \& Park, 1999 (Quassitagma); Insecta Koreana 16: 12. Holotype o*, Sri Lanka, Gal. District, Kanneliya, 200', 15-17 October 1976, black light, G. Hevel, Dietz, Karunaratne \& Balasooriya. USNM Genitalia Slide No. 89217. [Lecithoceridae]

lampyridella Busck, 1914 (Stenoma); Proceedings of the U.S. National Museum 47: 41. Holotype $o^{\star}$, Panama, Cabima, May 1911, A. Busck. USNM Type No. 16715, USNM Genitalia Slide No. 12773. [Elachistidae: Stenomatinae]

langei Keifer, 1936 (Gelechia); Bulletin of the Southern California Academy of Sciences 35: 20. Holotype ${ }^{\star}$, USA, California, near Placerville [no date]. [Gelechiidae: Gelechiinae] 
lanosa Duckworth, 1967 (Lethata); Proceedings of the U.S. National Museum 122: 10. Holotype $\sigma^{\star}$, Panama, Cocle Province, El Valle, 22 April 1965, S. S. \& W. D. Duckworth. W. D. Duckworth Genitalia Slide No. 3626. [Elachistidae: Stenomatinae]

lapilella Busck, 1914 (Catarata); Proceedings of the U.S. National Museum 47: 36. Holotype ${ }^{\star}$, Panama, Porto Bello, March 1912, A. Busck. USNM Type No. 16703, USNM Genitalia Slide No. 13855. [Elachistidae: Stenomatinae]

lasia Walsingham, 1912 (Zetesima); Biologia Centrali-Americana, Lepidoptera Heterocera 4: 157. Holotype $o^{\star}$, Panama, [no date], W. Schaus. USNM Genitalia Slide No. 14032, USNM Wing Slide No. 14033. [Elachistidae: Stenomatinae]

lassenella Busck, 1908 (Ethmia); Proceedings of the Entomological Society of Washington 9: 92. Lectotype ?, USA, Arizona, Redington [no date]. USNM Type No. 11332. Designated by Powell (1973). [Elachistidae: Ethmiinae]

lassula Hodges, 1962 (Ithome); Journal of the Lepidopterists' Society 15: 89. Holotype ox, USA, Florida, Key West, r.f. Leucaena glauca, em. 19 April 1945. USNM Type No. 4853. Most of specimen in gelatin capsule. [Cosmopterigidae: Chrysopeleiinae]

latebra Hodges, 1978 (Siskiwitia); Moths of America north of Mexico, fasc. 6.1: 112. Holotype ${ }^{\star}$, USA, Arkansas, Washington Co., Devil's Den State Park, 11 July 1966, R. W. Hodges. [Cosmopterigidae: Chrysopeleiinae]

latipalpella Barnes \& Busck, 1920 (Agonopteryx); Contributions to the Natural History of the Lepidoptera of North America 4: 233. Lectotype ${ }^{\star}$, USA, Texas, San Benito, 16-23 March. USNM Type No. 22669, USNM Genitalia Slide No. 394. Designated by Hodges (1974). [Elachistidae: Depressariinae]

latitans Dognin, 1905 (Cryptolechia); Annales de la Société Entomologique de Belgique 49: 89. Holotype ${ }^{\star}$, Brazil, Province de Saint-Paul, Paranapanema, [no date], Dognin Collection. USNM Type No. 32364, USNM Genitalia Slide No. 13872. [Elachistidae: Stenomatinae]

latro Hodges, 1999 (Chionodes); Moths of America north of Mexico, fasc. 7.6: 224. Holotype ${ }^{*}$, USA, Florida, Marion Co., Ocala National Forest, National Forest Road 75, Lake Delancy, 25 October 1992, J. S. Kutis. [Gelechiidae: Gelechiinae]

laudatella Walsingham, 1907 (Gelechia); Proceedings of the U.S. National Museum 33: 197. Holotype + , USA, California, Folsom [no date]. USNM Type No. 10677, USNM Genitalia Slide No. 10681. [Gelechiidae: Gelechiinae] 
lecaniella Busck, 1913 (Blastobasis); Insecutor Inscitiae Menstruus 1: 89. Holotype $\sigma^{*}$, 510 British Guiana, Plantation Nonpareil, r.f. Lecanium and Ceroplastes scales on old roots of lime and guava, [no date], H. W. B. Moore. USNM Type No. 16013, USNM Genitalia Slide No. 80989. [Coleophoridae: Blastobasinae]

lector Hodges, 1999 (Chionodes); Moths of America north of Mexico, fasc. 7.6: 121. Holotype $\diamond^{\star}$, USA, California, Riverside Co., Woodwardia Canyon E., 30 March 1947, r.f. Acer, em. 2 May 1947. USNM Genitalia Slide No. 8464. [Gelechiidae: Gelechiinae]

legnotoa Hodges, 1986 (Dichomeris); Moths of America north of Mexico, fasc. 7.1: 101. Holotype + , USA, Florida, Pinellas Co., Largo, 9 July 1982, E. Knudson. USNM Genitalia Slide No. 14662. [Gelechiidae: Dichomeridinae]

lenape Kaila, 1996 (Elachista); Entomologica Scandinavica 27: 237. Holotype o , USA, New Jersey, Lakehurst, 4 May 1962, R. W. Hodges. USNM Genitalia Slide No. 89285. [Elachistidae: Elachistinae]

lenta Clarke, 1956 (Ectaga); Entomological News 67: 253. Holotype ${ }^{*}$, Argentina, Tigre, 15 May 1954, F. Bourquin. USNM Type No. 63271, J. F. G. Clarke Genitalia Slide No. 10392. [Oecophoridae: Oecophorinae]

lenticula Clarke, 1986 (Asymphorodes); Smithsonian Contributions to Zoology 416: 220. Holotype $\sigma^{*}$, Marquesas Archipelago, Nuku Hiva, Tapuaooa, 2500', 30 January 1968, J. F. G. \& T. M. Clarke. USNM Type No. 100793, USNM Genitalia Slide No. 24899. [Cosmopterigidae: Cosmopteriginae]

lepidophaga Clarke, 1960 (Holcocera); Florida Entomologist 43: 115. Holotype ox, USA, Florida, Baker Co., 20 February 1958, em. 15 April 1958, r.f. ơ flower buds of Pinus elliotti, B. H. Ebel. USNM Type No. 64924, J. F. G. Clarke Genitalia Slide No. 10753. [Coleophoridae: Blastobasinae]

lepidotae Clarke, 1934 (Gelechia); Canadian Entomologist 65: 177. Holotype ơ, USA, Washington, Whitman Co., Snake River, opposite Clarkston, r.f. Glycyrrhiza lepidotae, H. H. Keifer. USNM Type No. 50179, USNM Genitalia Slide No. 9561. [Gelechiidae: Gelechiinae]

lepisma Walsingham, 1912 (Catarata); Biologia Centrali-Americana, Lepidoptera Heterocera 4: 154. Holotype + , Panama, Canal Zone, Tabernilla, June 1907, A. Busck. USNM Genitalia Slide No. 12964. Right forewing in gelatin capsule. [Elachistidae: Stenomatinae] 
leptotaeniae Clarke, 1933 (Depressaria); Canadian Entomologist 65: 87. Holotype ॰ $^{\star}$ USA, Washington, Pullman, 2 June 1932, r.f. Leptotaenia multifida Nutt., J. F. G. Clarke. USNM Type No. 44742. [Elachistidae: Depressariinae]

leptotes Clarke, 1986 (Asymphorodes); Smithsonian Contributions to Zoology 416: 274. Holotype $\sigma^{*}$, Marquesas Archipelago, Nuku Hiva, Tapuaooa, 2500', 30 January 1968, J. F. G. \& T. M. Clarke. USNM Type No. 100818, USNM Genitalia Slide No. 24806. [Cosmopterigidae: Cosmopteriginae]

lespedezae Walsingham, 1882 (Cosmopteryx); Proceedings of the Zoological Society of London, 10: 198. Holotype $\sigma^{*}$, r.f. Lespedeza, [no date], Riley Collection. According to Hodges (1962), the type probably came from Dallas, Texas, USA. [Cosmopterigidae: Cosmopteriginae]

leucaniella Busck, 1909 (Gelechia); Proceedings of the Entomological Society of Washington 11: 180. Holotype ${ }^{\star}$, USA, San Diego Co., San Diego, May, L. E. Ricksecker. USNM Type No. 12687, USNM Genitalia Slide No. 9928. [Gelechiidae: Gelechiinae]

leucocephala Becker, 1982 (Timocratica xanthosoma); Bulletin of the British Museum (Natural History), Entomology 45: 247. Holotype ${ }^{\star}$, Panama, Canal Zone, Barro Colorado Island, 5-10 April 1955, S. S. \& W. D. Duckworth. [Elachistidae: Stenomatinae]

leucoloma Clarke, 1986 (Asymphorodes); Smithsonian Contributions to Zoology 416: 251. Holotype + , Marquesas Archipelago, Hiva Oa, Mt. Feani, 3400', 1 March 1968, J. F. G. \& T. M. Clarke. USNM Type No. 100808, USNM Genitalia Slide No. 24993. [Cosmopterigidae: Cosmopteriginae]

leucomastis Diakokoff, 1968 (Lecithocera); Bulletin of the U.S. National Museum 257 (1967): 145. Holotype o, Philippine Islands, Luzon, Mt. Apo, 70 B, 9 May 1945, J. G. Franclemont. USNM Genitalia Slide No. 89974. [Lecithoceridae]

leuconotella Busck, 1904 (Trichotaphe); Proceedings of the U.S. National Museum 27: 762. Holotype + , USA, Washington, Pullman, Washington Experiment Station No. 477, 28 July 1898, C. V. Piper. USNM Type No. 7864, USNM Genitalia Slide No. 12346, USNM Wing Slide No. 12347. [Gelechiidae: Dichomeridinae]

leucoptera Clarke, 1986 (Autosticha); Smithsonian Contributions to Zoology 416: 171. Holotype ${ }^{\star}$, Marquesas Archipelago, Hiva Oa, Atuona, 27 February 1968, J. F. G \& T. M. Clarke. USNM Type No. 100770, USNM Genitalia Slide No. 24574. [Autostichidae: Autostichinae] 
leucothea Busck, 1914 (Stenoma); Proceedings of the U.S. National Museum 47: 50.

510 Holotype $\sigma^{*}$, Panama, Trinidad Island, March 1912, A. Busck. USNM Type No. 16735, USNM Genitalia Slide No. 13347. [Elachistidae: Stenomatinae]

leucotricha Clarke, 1971 (Semolina); Smithsonian Contributions to Zoology 56: 159. Holotype $\sigma^{*}$, Rapa Island, Haurei, 29 October 1963, J. F. G. \& T. M. Clarke. USNM Genitalia Slide No. 26268. [Cosmopterigidae: Cosmopteriginae]

leucoxantha Clarke, 1978 (Irenia); Smithsonian Contributions to Zoology 273: 45. Holotype ${ }^{\circ}$, Chile, Maule, Constitución, 26 November 1953, L. E. Peña. USNM Type No. 73714, USNM Genitalia Slide No. 24142. [Oecophoridae: Oecophorinae]

levisella Fyles, 1904 (Trichotaphe); Canadian Entomologist 36: 211. Lectotype o ${ }^{\star}$, Canada, Quebec, r.f. Aster macrophyllus, 10 July 1902, Levis. USNM Type No. 6813. Abdomen lacking. Designated by Hodges (1986). [Gelechiidae: Dichomeridinae]

libator Hodges, 1966 (Batrachedra); Transactions of the American Entomological Society 92: 628. Holotype ${ }^{\star}$, USA, Florida, Highlands Co., Parker Islands, 26-29 May 1964, R. W. Hodges. USNM Type No. 67867. [Batrachedridae]

linaria Clarke, 1957 (Batrachedra); Acta Zoologica Mexicana 2: 1. Holotype + , Mexico, Baja California, 1 January 1942, r.f. Agave leaf. USNM Type No. 63450, USNM Genitalia Slide No. 5103. [Batrachedridae]

linda Busck, 1914 (Ethmia); Insecutor Inscitiae Menstruus 2: 55. Holotype $\odot$, Venezuela, Caracas [no date]. USNM Type No. 18267. [Elachistidae: Ethmiinae]

lindanella Barnes \& Busck, 1920 (Aristotelia); Contributions to the Natural History of the Lepidoptera of North America 4: 226. Holotype + , USA, California, San Bernardino Co., Loma Linda [no date]. [Gelechiidae: Gelechiinae]

lindenella Busck, 1903 (Gelechia); Proceedings of the U.S. National Museum 25: 876. Holotype $\diamond^{\star}$, USA, Texas [no date]. USNM Type No. 6387, USNM Genitalia Slide No. 10683. [Gelechiidae: Gelechiinae]

lindseyi Barnes \& Busck, 1920 (Stenoma); Contributions to the Natural History of the Lepidoptera of North America 4: 239. Holotype o* USA, Arizona, Cochise Co., Paradise [no date]. W. D. Duckworth Genitalia Slide No. 2082. [Elachistidae: Stenomatinae] 
linealis Park \& Hodges, 1995 (Dichomeris); Insecta Koreana 12: 56. Holotype ${ }^{*}$, Taiwan, Taipei Co., Sozan, 10 June 1934, S. Issiki. USNM Genitalia Slide No. 11616. [Gelechiidae: Dichomeridinae]

lineola Clarke, 1978 (Deia); Smithsonian Contributions to Zoology 273: 12. Holotype 9 , Chile, Llanquihue, Petrohue, 10 March 1959, J. F. G. Clarke. USNM Type No. 73694, USNM Genitalia Slide No. 24207. [Oecophoridae: Oecophorinae]

liniella Busck, 1910 (Stenoma); Proceedings of the Entomological Society of Washington 12:80. Holotype ${ }^{x}$, Costa Rica, Sixola River, [no date], W. Schaus. USNM Type No. 13202, USNM Genitalia Slide No. 13753. [Elachistidae: Stenomatinae]

lipatiella Busck, 1909 (Gelechia); Proceedings of the Entomological Society of Washington 11: 88. Holotype ${ }^{\star}$, USA, Colorado, Platte Canyon, [no date], E. J. Oslar. USNM Type No. 12279, Genitalia Slide AB July 3, 1936. [Gelechiidae: Gelechiinae]

literatella Busck, 1911 (Pleurota); Proceedings of the U.S. National Museum 40: 205. Holotype $\alpha^{*}$, French Guiana, Rio Maroni, [no date], W. Schaus. USNM Type No. 13557, USNM Genitalia Slide No. 25766. [Oecophoridae: Oecophorinae]

lithochroma Busck, 1914 (Promenesta); Proceedings of the U.S. National Museum 47: Proceedings of the U.S. National Museum 47: 22. Holotype o*, Panama, Trinidad River, March 1912, A. Busck. USNM Type No. 16680, J. F. G. Clarke Genitalia Slide No. 4260. [Elachistidae: Stenomatinae]

lividula Park \& Hodges, 1995 (Dichomeris); Insecta Koreana 12: 57. Holotype $\sigma^{\star}$, Taiwan, Hualien Co., Pianau-col, 10 August 1943, A. Mutuura. USNM Genitalia Slide No. 87346. [Gelechiidae: Dichomeridinae]

loetae Clarke, 1942 (Chionodes); Proceedings of the U.S. National Museum 92: 270. Holotype $\sigma^{\star}$, USA, Washington, San Juan Co., Friday Harbor, 16 July 1926, T. C. Kincaid. USNM Type No. 56270, USNM Genitalia Slide No. 14576. [Gelechiidae: Gelechiinae]

lomionella Kaila, 1997 (Elachista); Acta Zoologica Fennica 206: 13. Holotype o*, USA, Massachusetts, Barnstable, 21 August 1952, C. P. Kimball. USNM Genitalia Slide No. 89289. [Elachistidae: Elachistinae]

lonchocarpella Busck, 1934 (Stilbosis); Entomologica Americana 13: 157. Holotype o*, Cuba, Santiago de las Vegas [no date]. USNM Genitalia Slide No. 4991. [Cosmopterigidae: Chrysopeleiinae] 

Holotype $\sigma^{\star}$, Marquesas Archipelago, Hiva Oa, Mt. Feani, 3800', 20 February 1968, J. F. G \& T. M. Clarke. USNM Type No. 100773. [Gelechiidae: Gelechiinae]

longimaculata Dognin, 1905 (Eumimographe); Annales de la Société Entomologique de Belgique 49: 87. Holotype $\sigma^{*}$, Ecuador, environs de Loja. USNM Type No. 32347, USNM Genitalia Slide No. 4078. [Oecophoridae: Oecophorinae]

longimaculella Chambers, 1872 (Hyponomeuta); Canadian Entomologist 4: 43. Pseudotype 9 , USA, Kentucky [no date]. USNM Type No. 413. [Elachistidae: Ethmiinae]

longitudinella Busck, 1914 (Besciva); Proceedings of the U.S. National Museum 47: 6. Holotype + , Panama, Trinidad River, March 1912, A. Busck. USNM Type No. 16670, USNM Genitalia Slide No. 10663. [Gelechiidae: Gelechiinae]

longivincula Wu \& Park, 1998 (Antiochtha); Insecta Koreana 15: 12. Holotype ơ, Sri Lanka, Kal. District, Agalawatta, 13-14 October 1976, black light, G. Hevel, Dietz, Karunaratne \& Balasooriya. USNM Genitalia Slide No. 88865. [Lecithoceridae]

lonicerae Zimmerman \& Bradley, 1950 (Swezeyula); Proceedings of the Hawaiian Entomological Society 14: 194. Holotype ${ }^{*}$, Hawaii, Makiki Valley, r.f. Lonicera japonica, 4 December 1949, O. H. Swezey. Abdomen in gelatin capsule. [Elachistidae: Elachistinae]

lophosella Busck, 1910 (Gelechia); Proceedings of the Entomological Society of Washington 11: 182. Holotype $\diamond^{\star}$, USA, California, San Diego Co., San Diego, May, L. E. Ricksecker. USNM Type No. 12689, USNM Genitalia Slide No. 9898. [Gelechiidae: Gelechiinae]

lososi Adamski \& Brown, 2002 (Blastobasis); Insecta Koreana 19: 142. Holotype ơ, Fiji, Viti Levu, South Pacific Bible College, 14 km W Lami, 7-10 December 1986, UV trap, R. L. \& B. B. Brown. USNM Genitalia Slide No. 81197. [Coleophoridae: Blastobasinae]

louisella Kaila, 1997 (Elachista); Acta Zoologica Fennica 206: 53. Holotype ${ }^{*}$, USA, California, Orange Co., Laguna Beach, 15-30 January 1925, O. C. Poling. USNM Genitalia Slide No. 89283. [Elachistidae: Elachistinae]

loxographa Diakonoff, 1968 (Philobota); Bulletin of the U.S. National Museum 257 (1967): 175. Holotype $\%$, Philippine Islands, Luzon, Mountain Province, Baguio, 9 October 1945, J. Franclemont. USNM Genitalia Slide No. 89977. [Oecophoridae: Oecophorinae] 
loxotoma Busck, 1909 (Stenoma); Proceedings of the Entomological Society of Washington 11: 212. Holotype $\sigma^{*}$, Mexico, Veracruz, Orizaba, [no date], R. Müller. USNM Type No. 12809, USNM Genitalia Slide No. 12585. [Elachistidae: Stenomatinae]

lucerna Clarke, 1986 (Asymphorodes); Smithsonian Contributions to Zoology 416: 249. Holotype $\sigma^{*}$, Marquesas Archipelago, Fatu Hiva, Tahuna, 2000', 27 March 1968, J. F. G. \& T. M. Clarke. USNM Type No. 100806, USNM Genitalia Slide No. 24990. [Cosmopterigidae: Cosmopteriginae]

lucidella Adamski, 1989 (Calosima); Mississippi Agricultural Forest Experimental Station Technical Bulletin 165: 18. Holotype o*, USA, Florida, Lake Placid, Archbold Biological Station, 1-7 May 1964, R.W. Hodges. USNM Genitalia Slide No. 81081. [Coleophoridae: Blastobasinae]

lucidella Busck, 1912 (Epicallima); Entomological News 23: 170. Holotype \&, USA, Pennsylvania, Allegheny Co., Oak Station, 15 June 1911, F. Marloff. USNM Genitalia Slide No. 1033. [Oecophoridae: Oecophorinae]

lucidus Clarke, 1986 (Asymphorodes); Smithsonian Contributions to Zoology 416: 244. Holotype $\sigma^{\star}$, Marquesas Archipelago, Hiva Oa, Atuina, 29 February 1968, J. F. G. \& T. M. Clarke. USNM Type No. 100802, USNM Genitalia Slide No. 24951. [Cosmopterigidae: Cosmopteriginae]

luminosa Busck, 1914 (Dichomeris); Proceedings of the U.S. National Museum 47: 19. Holotype $\propto^{\star}$, Panama, Portobello, March 1911, A. Busck. USNM Type No. 16676. Abdomen lacking. [Gelechiidae: Dichomeridinae]

lunata Adamski \& Brown, 2001 (Glyphidocera); Proceedings of the Entomological Society of Washington 103: 986. Holotype $\sigma^{*}$, Venezuela, Territorio Federal Amazona, San Carlos de Rio Negro, $1^{\circ} 56$ 'N, 6703'W, 6-12 December 1984, R. L. Brown. USNM Genitalia Slide No. 81639. [Glyphidoceridae]

lunimaculata Dognin, 1913 (Stenoma); Annales de la Société Entomologique de Belgique 57: 417. Holotype + , Colombia, [no date], Fassel, Dognin Collection. USNM Type No. 32363, USNM Genitalia Slide No. 17784. [Elachistidae: Stenomatinae]

lupinella Busck, 1901 (Anacampsis); Canadian Entomologist 33: 14. Holotype $\uparrow$, Canada, Ontario, Toronto, 10 June 1900, r.f. Lupinus perennis, em. 18 June 1900. USNM Type No. 5351, USNM Genitalia Slide No. 10658. [Gelechiidae: Gelechiinae] 

fornia, San Diego Co., La Puerta Valley [no date]. USNM Genitalia Slide No. 89281. [Elachistidae: Elachistinae]

luror Hodges, 1999 (Chionodes); Moths of America north of Mexico, fasc. 7.6: 193. Holotype $\sigma^{*}$, USA, Arizona, Coconino Co., West Fork, 16 mi SW Flagstaff, 6500', 15 July 1961, R. W. Hodges. USNM Genitalia Slide No. 7971. [Gelechiidae: Gelechiinae]

lushanae Park \& Hodges, 1995 (Dichomeris); Insecta Koreana 12: 22. Holotype o , Taiwan, Taipei Co., Sozan, 3 June 1934, S. Issiki. USNM Genitalia Slide No. 11561. Specimen not found. [Gelechiidae: Dichomeridinae]

lushanensis Park \& Wu, 2003 (Autosticha); Insecta Koreana 20: 206. Holotype o*, Taiwan, Nantou Co., Lu-shan, 30 km E Wu-she, ca. 1000 m, 27-31 May 1980, D. Davis. Specimen not found. [Autostichidae: Autostichinae]

lutea Park \& Hodges, 1995 (Dichomeris); Insecta Koreana 12: 50. Holotype ${ }^{*}$, Taiwan, Nantou Co., Meifeng, 30 km S Tayuling, 2200 m, 8 June 1980, D. Davis. USNM Genitalia Slide No. 87355. [Gelechiidae: Dichomeridinae]

luteogeminatus Clarke, 1935 (Gelechia); Canadian Entomologist 67: 251. Holotype $\sigma^{*}$, USA, Washington, Whitman Co., Wawawai, 3 August 1934, J. F. G. Clarke. USNM Type No. 50944, USNM Genitalia Slide No. 7711. [Gelechiidae: Gelechiinae]

luteola Diakonoff, 1968 (Lecithocera); Bulletin of the U.S. National Museum 257 (1967): 145. Holotype ${ }^{x}$, Philippine Islands, Luzon, Agoo, La Union, 1 May 1945, J. G. Franclemont. USNM Genitalia Slide No. 89973. [Lecithoceridae]

lutescella Clarke, 1934 (Gnorimoschema); Canadian Entomologist 65: 172. Holotype ơ, USA, Washington, Pullman, r.f. Castilleja lutescens [no date]. USNM Type No. 50177, USNM Genitalia Slide No. 7206. [Gelechiidae: Gelechiinae]

macelhosiella Busck, 1907 (Ethmia); Proceedings of the Entomological Society of Washington 8: 93. Holotype ${ }^{*}$, USA, Missouri, St. Louis, [no date], H. A. McElhose. USNM Type No. 9898. Caudal portion of abdomen damaged, genitalia lacking. [Elachistidae: Ethmiinae]

macrogramma Clarke, 1986 (Asymphorodes); Smithsonian Contributions to Zoology 416: 186. Holotype $\sigma^{\star}$, Marquesas Archipelago, Nuku Hiva, 30 January 1968, J. F. G. \& T. M. 
Clarke. USNM Type No. 100774, USNM Genitalia Slide No. 25140. [Cosmopterigidae: Cosmopteriginae]

maculata Clarke, 1978 (Altiura); Smithsonian Contributions to Zoology 273: 19. Holotype 9 , Chile, Santiago Province, Cajon de Maypo, Cordillera, El Canelo, 12-20 January 1948, T. Ramirez. USNM Type No. 73698, USNM Genitalia Slide No. 24206. [Oecophoridae: Oecophorinae]

maculata Duckworth, 1964 (Lethata); Proceedings of the U.S. National Museum 116: 106. Holotype $o^{*}$, Brazil, Nova Teutônia, 3500 m, 27¹1'8"S, 52²3' W, 21 October 1948, F. Plaumann. USNM Genitalia Slide No. 13392. [Elachistidae: Stenomatinae]

maculatella Busck, 1908 (Depressaria); Proceedings of the Entomological Society of Washington 9: 90. Lectotype $\sigma^{*}$ Canada, Ontario, [no date], A. W. Hanham. USNM Type No. 11329, USNM Genitalia Slide No. 695. Designated by Hodges (1974). [Elachistidae: Depressariinae]

magnatella Busck, 1904 (Scythris); Proceedings of the U.S. National Museum 27: 768. Holotype $\sigma^{*}$, Canada, British Columbia, Kaslo [no date]. USNM Type No. 7884, USNM Genitalia Slide No. 15856. [Xyloryctidae: Scythridinae]

magnella Busck, 1903 (Paltodora); Proceedings of the U.S. National Museum 25: 776. Holotype ${ }^{\star}$, USA, Colorado [no date]. USNM Type No. 6345, USNM Genitalia Slide No. 5598. [Gelechiidae: Gelechiinae]

major Busck, 1911 (Stenoma); Proceedings of the U.S. National Museum 40: 212. Holotype ?, Peru, Callao, [no date], M. J. Pusey. USNM Type No. 13567, USNM Genitalia Slide No. 12623. [Elachistidae: Stenomatinae]

major Kearfott, 1907 (Antispila); Bulletin of the American Museum 23: 166. Holotype ox, USA, North Carolina, Black Mountain, 8 June, W. Beutenmüller. USNM Genitalia Slide No. 98386. [Elachistidae: Elachistinae]

malindella Busck, 1910 (Gelechia); Proceedings of the Entomological Society of Washington 11: 179. Holotype ${ }^{*}$, USA, New Mexico, Mesilla, [no date], C. N. Ainsley. USNM Type No. 12685, USNM Genitalia Slide No. 7608. [Gelechiidae: Gelechiinae]

mandella Busck, 1904 (Gelechia); Proceedings of the U.S. National Museum 27: 759. Holotype $\diamond^{*}$, Canada, British Columbia, Kaslo, August, Dyar. USNM Type No. 7859, USNM Genitalia Slide No. 6775. [Gelechiidae: Gelechiinae] 

type ${ }^{\star}$, USA, Illinois, Putnam Co., 19 August 1965, M. O. Glenn. USNM Genitalia Slide No. 8827. [Cosmopterigidae: Chrysopeleiinae]

mansita Busck, 1914 (Ethmia); Insecutor Inscitiae Menstruus 2: 55. Holotype ${ }^{*}$, Mexico, Tehuacan, September 1913, R. Müller. USNM Type No. 18266. [Elachistidae: Ethmiinae]

marcella Busck, 1914 (Cryptolechia); Proceedings of the U.S. National Museum 47: 29. Holotype ${ }^{\star}$, Panama, Trinidad River, March 1912, A. Busck. USNM Type No. 16750, USNM Genitalia Slide No. 69472. [Elachistidae: Depressariinae]

marginata Busck, 1914 (Stenoma); Proceedings of the U.S. National Museum 47: 49. Holotype + , Panama, Trinidad River, March 1912, A. Busck. USNM Type No. 16729. Abdomen lacking. [Elachistidae: Stenomatinae]

marginella Busck, 1914 (Promenesta); Proceedings of the U.S. National Museum 47: 22. Holotype $\sigma^{*}$ Panama, Trinidad River, May 1911, A. Busck. USNM Type No. 16681, USNM Genitalia Slide No. 14361, USNM Wing Slide No. 14362. [Elachistidae: Stenomatinae]

mariona Heinrich, 1921 (Telphusa); Journal of Agricultural Research 20: 812. Holotype o, USA, Texas, Brownsville, 20 April 1919, r.f. Abutilon incanum. USNM Type No. 23740, USNM Genitalia Slide No. 8213 [Gelechiidae: Gelechiinae]

maroni Busck, 1911 (Stenoma); Proceedings of the U.S. National Museum 40: 220. Holotype + , French Guiana, St. Jean, Maroni River, [no date], W. Schaus. USNM Type No. 13583, USNM Genitalia Slide No. 13048. [Elachistidae: Stenomatinae]

maroniella Busck, 1911 (Peleopoda); Proceedings of the U.S. National Museum 40: 208. Holotype $\sigma^{x}$, French Guiana, St. Jean, Maroni River, [no date], W. Schaus. USNM Type No. 13561, USNM Genitalia Slide No. 69540. [Peleopodidae]

mathesoni Busck, 1916 (Batrachedra); Proceedings of the Entomological Society of Washington 18: 150. Lectotype + , USA, Florida, Coconut Grove, r.f. blossoms of coconut (Cocos nucifera), em. 20 May 1916, H. M. Matheson. USNM Type No. 20336, USNM Genitalia Slide No. 5132. Designated by Hodges (1966). [Batrachedridae]

mcdunnoughi Clarke, 1941 (Semioscopis); Proceedings of the U.S. National Museum 90: 162. Holotype $\sigma^{*}$, USA, Washington, Bellingham, 14 March 1923, J. F. G. Clarke. USNM Type No. 53117, USNM Genitalia Slide No. 490. [Elachistidae: Depressariinae] 
meddix Hodges, 1999 (Chionodes); Moths of America north of Mexico, fasc. 7.6: 73. Holotype $\$$, USA, Arizona, Yavapai Co., Clear Creek Camp, SE Camp Verde, 17 June 1968, O. Flint \& A. Menke. USNM Genitalia Slide No. 11912. [Gelechiidae: Gelechiinae]

mediella Busck, 1913 (Ethmia); Journal of Entomology and Zoology (Pomona) 5: 99. Syntype 9 , USA, California, San Diego, 4 June 1911, W. S. Wright. USNM Type No. 15612. There are several additional syntypes in main collection of USNM. [Elachistidae: Ethmiinae]

mediostriatus Clarke, 1986 (Asymphorodes); Smithsonian Contributions to Zoology 416: 274. Holotype $\sigma^{*}$, Marquesas Archipelago, Nuku Hiva, Tunoa Ridge, 2900', 23 January 1968, J. F. G. \& T. M. Clarke. USNM Type No. 100819. [Cosmopterigidae: Cosmopteriginae]

medulella Busck, 1914 (Telphusa); Proceedings of the U.S. National Museum 47: 15. Holotype ơ, Panama, Porto Bello, April 1912, A. Busck. USNM Type No. 15830. [Gelechiidae: Gelechiinae]

megamicrella Dyar, 1902 (Semioscopis); Canadian Entomologist 34: 320. Syntype o*, USA, Pennsylvania, New Brighton, 21 March 1902, H. D. Merrick. USNM Type No. 6611, USNM Genitalia Slide No. 2239. This species was described from 8 syntypes; we were unable to locate the other 7 specimens. [Elachistidae: Depressariinae]

meibomiella Forbes, 1931 (Anacampsis); Journal of the Department of Agriculture, Porto Rico 15: 376. Holotype ${ }^{\star}$, Puerto Rico, “E.E.A. de Cuba, No. 8454, on Meybomia." [Gelechiidae: Gelechiinae]

melaina Clarke, 1986 (Chelophoba); Smithsonian Contributions to Zoology 416: 173. Holotype ${ }^{\star}$, Marquesas Archipelago, Fatu Hiva, Omoa, 18 March 1968, J. F. G. \& T. M. Clarke. USNM Type No. 100771, USNM Genitalia Slide No. 24572. Right fore- and hindwing lacking. [Gelechiidae: Gelechiinae]

melaleuca Clarke, 1965 (Anchimompha); Proceedings of the U.S. National Museum 117: 90. Holotype 9 , Chile, Juan Fernandez Islands, Masafuera, Quebrada de las Casas, 7 February 1955, P. G. Kuschel. USNM Genitalia Slide No. 87691. Right fore- and hindwing lacking. [Coleophoridae: Momphinae]

melanella Murtfeldt, 1890 (Menesta); Insect Life 2: 304. Holotype ${ }^{*}$, USA, Missouri, r.f. Quercus, 16 March 1891. [Elachistidae: Stenomatinae] 
melanocrepida Clarke, 1962 (Psorosticha); Entomological News 73: 91. Holotype o , Japan, Kyusyu, Oita, r.f. Citrus unshiu, 3 June 1957, K. Yasumatsu. USNM Type No. 65828, J. F. G. Clarke Genitalia Slide No. 10729. [Elachistidae: Depressariinae]

melanolepis Clarke, 1965 (Gnorimoschema); Proceedings of the U.S. National Museum 117: 83. Holotype ${ }^{*}$, Chile, Juan Fernandez Islands, Masafuera, Quebrada de las Casas, 22 January 1955. J. F. G. Clarke Genitalia Slide No. 10751. [Gelechiidae: Gelechiinae]

melanoma Clarke, 1978 (Aniuta); Smithsonian Contributions to Zoology 273: 7. Holotype *, Chile, Llanquihue, Peulla, 7 March 1959, J. F. G. Clarke. USNM Type No. 73692, USNM Genitalia Slide No. 24240. [Oecophoridae: Oecophorinae]

melanostriga Becker, 1982 (Timocratica); Bulletin of the British Museum (Natural History), Entomology 45: 266. Holotype 9 , Brazil, Santa Catarina, F. Hoffmann. W. D. Duckworth Genitalia Slide No. 2016. [Elachistidae: Stenomatinae]

melanota Walsingham, 1911 (Dichomeris); Biologia Centrali-Americana, Lepidoptera Heterocera 4: 94. Holotype + , Mexico, Veracruz, Cordoba, April 1908, F. Knab. Abdomen and right forewing in gelatin capsules. [Gelechiidae: Dichomeridinae]

melantherella Busck, 1900 (Trichotaphe), Proceedings of the U.S. National Museum 23: 232. Lectotype ${ }^{*}$, USA, Florida, Palm Beach, r.f. Melanthera deltoidea, em. 13 March 1900. USNM Type No. 4939, USNM Genitalia Slide No. 10549. Designated by Hodges (1986). [Gelechiidae: Dichomeridinae]

melissia Walsingham, 1911 (Dichomeris); Biologia Centrali-Americana, Lepidoptera Heterocera 4: 97. Holotype ${ }^{*}$, Panama, Canal Zone, Tabernilla, June 1907, A. Busck. J. F. G. Clarke Genitalia Slide No. 5067. [Gelechiidae: Dichomeridinae]

mendoron Busck, 1911 (Stenoma); Proceedings of the U.S. National Museum 40: 214. Holotype $\sigma^{*}$, French Guiana, St. Jean, Maroni River, [no date],W. Schaus. USNM Type No. 13570, USNM Genitalia Slide No. 13152. [Elachistidae: Stenomatinae]

meniscogramma Clarke, 1978 (Philomusea); Smithsonian Contributions to Zoology 273: 71. Holotype 9 , Chile, Llanquihue, Puerto Varas, 5 March 1959, J. F. G. Clarke. USNM Type No. 73728, USNM Genitalia Slide No. 24205. Right fore- and hindwing lacking. [Oecophoridae: Oecophorinae]

mentzeliae Clarke, 1942 (Anoncia); Canadian Entomologist 74: 18. Holotype ơ, USA, Washington, Whitman Co., Snake River, opposite Clarkeston, 15 August 1940, J. F. G. Clarke. USNM Type No. 55727. [Cosmopterigidae: Cosmopteriginae] 
menura Clarke, 1971 (Gonionota); Smithsonian Contributions to Zoology 95: 18. Holotype 9 , Panama, Cerro Campana, near Chica, 2-5 April 1965, S. S. \& W. D. Duckworth. USNM Type No. 71095, USNM Genitalia Slide No. 68716. [Oecophoridae: Oecophorinae]

mercatrix Hodges, 1986 (Dichomeris); Moths of America north of Mexico, fasc. 7.1: 110. Holotype + , USA, New York, Tompkins Co., McLean Bogs Reserve, 18 July 1963, J. G. Franclemont. USNM Genitalia Slide 12352. [Gelechiidae: Dichomeridinae]

merinxa Wu \& Park, 1998 (Hygroplasta); Insecta Koreana 15: 10. Holotype ${ }^{*}$, Sri Lanka, Rat. District, Sinharaja Jungle, 2 mi S of Weddagala, 8-12 February, 1977, malaise trap, Krombein, Fernando, Balasooriya \& Gunawardane. USNM Genitalia Slide No. 89212. [Lecithoceridae]

merista Clarke, 1971 (Autosticha); Smithsonian Contributions to Zoology 56: 143. Holotype 9 , Rapa Island, Haurei, 9 December 1963, J. F. G. \& T. M. Clarke. USNM Genitalia Slide No. 24570. [Autostichidae: Autostichinae]

merriccella Dyar, 1902 (Semioscopis); Canadian Entomologist 34: 319. Syntype o*, USA, Pennsylvania, New Brighton, 15 March 1902, H. D. Merrick. USNM Type No. 6609, USNM Genitalia Slide No. 459. This species was described from 2 specimens; we were unable to locate the other syntype. [Elachistidae: Depressariinae]

mesosura Wu \& Park, 1999 (Lecithocera); Insecta Koreana 16 (1): 9. Holotype ${ }^{\star}$, Sri Lanka, Rat. District, Uggalkaltota, Irrigation Bungalow, 350', 31 January-8 February 1970, D. Davis \& B. Rowe. USNM Genitalia Slide No. 88827. [Lecithoceridae]

metallifera Walsingham, 1882 (Elachista); Transactions of the American Entomological Society 1882: 200. Holotype ${ }^{\star}$, USA, Massachusetts, Amherst, “1076," Fernald Collection. USNM Genitalia Slide No. 86610. [Coleophoridae: Momphinae]

metaxea Kaila, 1995 (Mendesia); Journal of the Lepidopterists' Society 49: 221. Holotype o, USA, Texas, Brewster Co., Chisos Mts., K-Bar Ranch, 3400', 1 June 1973, R. W. Hodges. USNM Genitalia Slide No. 89302. [Elachistidae: Elachistinae]

metoecus Hodges, 1999 (Chionodes); Moths of America north of Mexico, fasc. 7.6: 125. Holotype ${ }^{*}$, USA, Utah, Wasatch Co., Snake Creek, 3 mi NW Midway, 1 August 1971, D. C. Ferguson. [Gelechiidae: Gelechiinae]

metopaena Wu \& Park, 1999 (Lecithocera); Insecta Koreana 16 (1): 4. Holotype o*, Sri Lanka, Anu. District Hunuwilagama, near Wilpattu, 200', at black light, 28 October-3 November 1976, Hevel, Dietz, Karunaratne \& Balasooriya. USNM Genitalia Slide No. 88900. [Lecithoceridae] 
mexicana Landry, 1994 (Coleophora); Canadian Entomologist 126: 1189. Holotype $\sigma^{*}$, Mexico, Tamaulipas, Gomes Farias, 1000 m, 29-31 July 1988, V. O. Becker \& M. A. Solis. USNM Genitalia Slide No. 87593. [Coleophoridae: Coleophorinae]

meyrickella Busck, 1907 (Glyphidocera); Journal of the New York Entomological Society 15: 138. Holotype $\sigma^{*}$, USA, Maryland, Montgomery Co., Plummers Island, August 1903, A. Busck. USNM Type No. 10321, USNM Genitalia Slide No. 10638. [Glyphidoceridae]

mica Hodges, 1986 (Dichomeris); Moths of America north of Mexico, fasc. 7.1: 84. Holotype $\sigma^{\star}$, USA, Texas, Brewster Co., Chisos Mountains, Panther Pass, 6000', 2 June 1973, R. W. Hodges. [Gelechiidae: Dichomeridinae]

microsema Clarke, 1986 (Iressa); Smithsonian Contributions to Zoology 416: 293. Holotype + , Marquesas Archipelago, Fatu Hiva, Omoa, 18 March 1968, J. F. G. \& T. M. Clarke. USNM Type No. 100822. [Cosmopterigidae: Cosmopteriginae]

mikkolai Hodges, 1999 (Chionodes); Moths of America north of Mexico, fasc. 7.6: 208. Holotype $\sigma^{\star}$, Canada, Yukon, Carmacks, 16 July 1985, K. Mikkola. USNM Genitalia Slide No. 87200. [Gelechiidae: Gelechiinae]

miltopeza Clarke, 1978 (Doshia); Smithsonian Contributions to Zoology 273: 36. Holotype $\sigma^{x}$, Chile, San Ignacio, November 1892, V. Izquerdo. USNM Type No. 73711, USNM Genitalia Slide No. 24266, USNM Wing Slide No. 24217. [Oecophoridae: Oecophorinae]

mimesis Hodges, 1986 (Dichomeris); Moths of America north of Mexico, fasc. 7.1: 101. Holotype $\sigma^{\star}$, USA, Texas, Anderson Co., Salmon, malaise trap, 22 July-2 August 1974, H. R. Burke. USNM Genitalia Slide No. 86849. [Gelechiidae: Dichomeridinae]

miniata Dognin, 1905 (Cryptolechia); Annales de la Société Entomologique de Belgique 49: 88. Holotype ơ, Colombia, Popayan, 1895, W. Schaus. USNM Genitalia Slide No. 69431. [Elachistidae: Depressariinae]

minna Busck, 1914 (Gonioterma); Proceedings of the U.S. National Museum 47: 52. Holotype $\sigma^{\star}$, Panama, Porto Bello, March 1911, A. Busck. USNM Type No. 16741, USNM Genitalia Slide No. 13018. [Elachistidae: Stenomatinae]

minor Busck, 1906 (Phthorimaea); Proceedings of the U.S. National Museum 30: 726. Syntypes ( $1 \sigma^{*}, 1$ uncertain sex), USA, Texas [no date]. USNM Type No. 9770. Both specimens lacking abdomen. [Gelechiidae: Gelechiinae] 
minor Busck, 1914 (Stenoma); Proceedings of the U.S. National Museum 47: 49. Holotype

o, Panama, Trinidad River, March 1912, A. Busck. USNM Type No. 16730, USNM Genitalia Slide No. 13879. [Elachistidae: Stenomatinae]

minuta Busck, 1914 (Decantha); Proceedings of the U.S. National Museum 47: 31. Holotype $\sigma^{*}$, Panama, Trinidad River, March, A. Busck. USNM Type No. 16755. [Oecophoridae: Oecophorinae]

minutella Beutenmüller, 1889 (Cosmopteryx); Entomologica Americana 5: 10. Holotype ^, USA, Florida [no date]. USNM Type No. 497. Abdomen lacking. [Cosmopterigidae: Cosmopteriginae]

miranda Clarke, 1965 (Pseudarla); Proceedings of the U.S. National Museum 117: 83. Holotype $\sigma^{*}$, Chile, Juan Fernandez Islands, Masatierra, Bahia Cumberland, 7 January 1955. J. F. G. Clarke Genitalia Slide No. 10591. [Gelechiidae: Gelechiinae]

miriella Kaila, 1999 (Elachista); Acta Zoologica Fennica 211: 83. Holotype $\uparrow$, USA, Colorado, Chaffee Co., 4 mi SW Buena Vista, 8700', pure stand ponderosa pine, 2 July 1982 , R. W. Hodges. USNM Genitalia Slide No. 90104. [Elachistidae: Elachistinae]

miscecalonella Chambers, 1875 (Laverna); Canadian Entomologist 7: 34. Holotype o*, USA, Texas [no date]. [Gelechiidae: Chrysopeleiinae]

miscitatella Clarke, 1932 (Gnorimoschema); Canadian Entomologist 64: 65. Holotype $\sigma^{*}$, USA, Washington, Wenatchee, May-September, A. Spuler. USNM Type No. 43452, USNM Genitalia Slide No. 7266. [Gelechiidae: Gelechiinae]

miseta Walsingham, 1913 (Stenoma); Biologia Centrali-Americana, Lepidoptera Heterocera 4: 180. Holotype + , Costa Rica, Peralta, 2000', February 1907, W. Schaus. USNM Genitalia Slide No. 14089. [Elachistidae: Stenomatinae]

mistipalpis Walsingham, 1911 (Dichomeris); Biologia Centrali-Americana, Lepidoptera Heterocera 4: 102. Holotype ox, Panama, Canal Zone, Tabernilla, June 1907, A. Busck. Abdomen lacking. [Gelechiidae: Dichomeridinae]

mistrella Busck, 1907 (Stenoma); Proceedings of the Entomological Society of Washington 8: 93. Holotype ơ, USA, Missouri, $2 \mathrm{mi}$ SW St. Louis [no date]. USNM Type No. 10708, Genitalia Slide AB Mar. 20, 1928. [Elachistidae: Stenomatinae] 
moira Clarke, 1984 (Elaeonoma); Insects of Micronesia 9: 145. Holotype $\sigma^{\star}$, Micronesia, Eastern Caroline Island, Ponape, $2.5 \mathrm{mi}$ E Colonia, low elevation, 15 December 1976, J. F. G. \& T. M. Clarke. USNM Type No. 76071, USNM Genitalia Slide No. 24374. [Oecophoridae: Oecophorinae]

molitor Hodges, 1999 (Chionodes); Moths of America north of Mexico, fasc. 7.6: 210. Holotype ${ }^{*}$, USA, Illinois, 7 June 1972, M. O. Glenn. [Gelechiidae: Gelechiinae]

mollis Barnes \& Busck, 1920 (Dichomeris); Contributions to the Natural History of the Lepidoptera of North America 4: 230. Holotype $\sigma^{*}$, USA, Arizona, Redington [no date]. USNM Genitalia Slide No. 87167. [Gelechiidae: Dichomeridinae]

monachella Busck, 1910 (Ethmia); Proceedings of the Entomological Society of Washington 12: 53. Holotype ${ }^{*}$, USA, Colorado, Boulder, [no date], S. A. Rohwer. USNM Type No. 12883, USNM Genitalia Slide No. 98447. [Elachistidae: Ethmiinae]

monella Busck, 1904 (Gelechia); Proceedings of the U.S. National Museum 27: 759. Syntypes $\left(3{ }^{x}\right)$, Canada, British Columbia, Kaslo, August, H. Dyar. USNM Type No. 7860, USNM Genitalia Slide No. 6772, USNM Wing Slide No. 6778. Abdomen lacking from 2 specimens. [Gelechiidae: Gelechiinae]

mones Hodges, 1978 (Anoncia); Moths of America north of Mexico, fasc. 6.1: 63. Holotype $\sigma^{*}$, USA, Texas, Culberson Co., Guadalupe Mountains, Cherry Canyon, 5096', 22 May 1973, R. W. Hodges. USNM Genitalia Slide No. 4624. [Cosmopterigidae: Cosmopteriginae]

monila Wu \& Park, 1998 (Hygroplasta); Insecta Koreana 15: 8. Holotype o*, Sri Lanka, Gal. District, Kanneliya Jungle, 11-16 January 1975, malaise trap, Krombien, Karunaratne, Fernando \& Weragoda. USNM Genitalia Slide No. 89218. [Lecithoceridae]

monilella Barnes \& Busck, 1920 (Aristotelia); Contributions to the Natural History of the Lepidoptera of North America 4: 225. Holotype $q$, USA, North Carolina, Southern Pines [no date]. USNM Genitalia Slide No. 5894. [Gelechiidae: Gelechiinae]

monocolona Meyrick, 1932 (Antaeotricha); Exotic Microlepidoptera 4: 293. Holotype $\sigma^{\star}$, Bolivia, Cochabamba, Incachaca, [no date], J. Steinbach. Abdomen lacking. [Elachistidae: Stenomatinae] 
monopthalma Clarke, 1971 (Batrachedra); Smithsonian Contributions to Zoology 56: 162. Holotype $\diamond^{\star}$, Rapa Island, Haurei, r.f. Cyperus javanicus, em. 27 November 1963, J. F. G. \& T. M. Clarke. USNM Type No. 70097, USNM Genitalia Slide No. 26273. [Batrachedridae]

monotaeniella Botttimer, 1926 (Gelechia); Journal of Agricultural Research 33: 813. Holotype $\sigma^{x}$, USA, Texas, Chambers Co., N shore Lake Charlotte, r.f. Vachellia farnesiana, 12 November 1923. USNM Type No. 27337, USNM Genitalia Slide No. 9570. [Gelechiidae: Gelechiinae]

moorei Busck, 1913 (Arauzona); Insecutor Inscitiae Menstruus 1: 88. Holotype ${ }^{*}$, British Guiana, r.f. Pisonia incramis, [no date], Craig. USNM Type No. 16011, Genitalia Slide AB Jan. 13, 1930. [Oecophoridae: Stathmopodinae]

moya Clarke, 1947 (Depressaria); Journal of the Washington Academy of Sciences 37: 13. Holotype $\sigma^{\star}$, USA, California, Siskiyou Co., Hornbrook, 7 May 1944, r.f. Lomatium vaginatum, J. F. G. Clarke. USNM Type No. 58011, USNM Genitalia Slide No. 639. [Elachistidae: Depressariinae]

mucida Duckworth, 1967 (Lethata); Proceedings of the U.S. National Museum 122: 17. Holotype $\sigma^{\star}$, Colombia, Dept. Santander, San Jaoquin, 30 August 1965, W. D. Duckworth. USNM Genitalia Slide No. 13349. [Elachistidae: Stenomatinae]

mulleri Busck, 1910 (Ethmia); Proceedings of the Entomological Society of Washington 11: 212. Holotype ${ }^{\star}$, Mexico, Tehuacan, [no date], R. Müller. USNM Type No. 12810. [Elachistidae: Ethmiinae]

mulsa Hodges, 1986 (Dichomeris); Moths of America north of Mexico, fasc. 7.1: 83. Holotype ${ }^{*}$, USA, Arizona, Santa Cruz Co., Santa Rita Mountains, Madera Canyon, 5600', 28 June 1973, J. G. Franclemont. [Gelechiidae: Dichomeridinae]

multifasciella Chambers, 1875 (Theisoa); Canadian Entomologist 7: 93. Syntype (uncertain sex), USA, Texas. There is one syntype in USNM and one in MCZ. Specimen not found. [Gelechiidae: Gelechiinae]

multifidae Clarke, 1933 (Depressaria); Canadian Entomologist 65: 85. Holotype ${ }^{\star}$, USA, Washington, Whitman Co., Snake River, opposite Clarkston, 19 May 1932, J. F. G. Clarke. USNM Type No. 44741. [Elachistidae: Depressariinae]

mumiella Adamski \& Brown, 2001 (Glyphidocera); Proceedings of the Entomological Society of Washington 103: 991. Holotype $\sigma^{*}$, Venezuela, Territorio Federal Amazona, 
munifex Hodges, 1999 (Chionodes); Moths of America north of Mexico, fasc. 7.6: 76. Holotype ${ }^{*}$, USA, Arizona, Santa Cruz Co., Santa Rita Mountains, Madera Canyon, 13 June 1963, J. G. Franclemont. [Gelechiidae: Gelechiinae]

murcyae Adamski \& Brown, 2002 (Blastobasis); Insecta Koreana 19: 138. Holotype ox, New Caledonia, Noumea, Naie Tina, 14-15 February 1984, M. Pogue \& M. Epstein. USNM Genitalia Slide No. 81452. [Coleophoridae: Blastobasinae]

muricolorella Busck, 1902 (Depressaria); Proceedings of the U.S. National Museum 24: 741. Holotype (uncertain sex), USA, Colorado, Golden [no date]. Specimen not found. [Oecophoridae: Depressariinae]

naclia Hodges, 1978 (Anoncia); Moths of America north of Mexico, fasc. 6.1: 64. Holotype $\sigma^{*}$, USA, Arizona, Pima Co., Baboquivari Mountains, 1-15 July 1924, O. C. Poling. USNM Genitalia Slide No. 4112. [Cosmopterigidae: Cosmopteriginae]

nadia Clarke, 1950 (Ethmia); Journal of the Washington Academy of Sciences 40: 161. Holotype ${ }^{\star}$, USA, California, McCloud, 5 June 1935. USNM Type No. 59637, USNM Genitalia Slide No. 10860. [Elachistidae: Ethmiinae]

naevus Hodges, 1999 (Chionodes); Moths of America north of Mexico, fasc. 7.6: 77. Holotype $\sigma^{*}$, USA, Arizona, Cochise Co., Chiricahua Mountains, Cave Creek Canyon, 5400', 18 May 1966, J. G. Franclemont. [Gelechiidae: Gelechiinae]

nana Dietz, 1910 (Holcocera); Transactions of the American Entomological Society 36: 48. Holotype $\%$, USA, Texas, Brownsville, [no date], H. S. Barber. Abdomen lacking. [Coleophoridae: Blastobasinae]

nancyae Clarke, 1990 (Mompha); Journal of the Lepidopterists' Society 44: 253. Holotype \%, Canada, British Columbia, Queen Charlotte Islands, Moresby Island, sandspit, 21 July 1987, J. F. G. Clarke \& N. L. duPre. USNM Genitalia Slide No. 69785. [Coleophoridae: Momphinae]

nanodella Busck, 1910 (Gelechia); Proceedings of the Entomological Society of Washington 11: 179. Holotype ${ }^{*}$, USA, California, San Diego Co., San Diego, May, L. E. Ricksecker. USNM Type No. 12684, USNM Genitalia Slide No. 9897. [Gelechiidae: Gelechiinae] 
natalella Busck, 1904 (Aristotelia); Proceedings of the U.S. National Museum 27: 756. Holotype ${ }^{\star}$, Canada, British Columbia, Kalso, July, Dyar \& Cockle. USNM Type No. 7854, USNM Genitalia Slide No. 5968. [Gelechiidae: Gelechiinae]

nathrax Hodges, 1974 (Callima); Moths of America north of Mexico, fasc. 6.2: 110. Holotype 9 , USA, Florida, Lake Placid, Archbold Biological Station, 16-22 May 1964, R. W. Hodges. [Oecophoridae: Oecophorinae]

neacantha Wu \& Park, 1999 (Frisilia); Korean Journal of Systematic Zoology 15: 6. Holotype $\sigma^{\star}$, Sri Lanka, Kan. District, Udawattakele, 2100', 5 October 1976, Hevel, Dietz, Karunaratne \& Balasooriya. USNM Genitalia Slide No. 89614. [Lecithoceridae]

nebrias Clarke, 1986 (Asymphorodes); Smithsonian Contributions to Zoology 416: 277. Holotype $\sigma^{*}$, Marquesas Archipelago, Nuku Hiva, Tunoa Ridge, 2900', 23 January 1968, J. F. G. \& T. M. Clarke. USNM Type No. 100820, USNM Genitalia Slide No. 24840. [Cosmopterigidae: Cosmopteriginae]

nebulombra Powell, 1973 (Ethmia semiombra); Smithsonian Contributions to Zoology 120: 190. Holotype $\sigma^{*}$, Mexico, Yucatán, Merida, 29-30 July 1964, P. J. Spangler. [Elachistidae: Ethmiinae]

nechlys Hodges, 1974 (Martyrhilda); Moths of America north of Mexico, fasc. 6.2: 46. Holotype 9 , USA, Arizona, Yuma Co., Planet Mine, [no date], B. Williams. USNM Genitalia Slide No. 943. [Elachistidae: Depressariinae]

nefrax Hodges, 1974 (Borkhausenia); Moths of America north of Mexico, fasc. 6.2: 124. Holotype $\odot^{\star}$, USA, Arizona, Coconino Co., $7.5 \mathrm{mi}$ NW Flagstaff, Fort Valley, 7350', 22 August 1961, R. W. Hodges. R. Hodges Genitalia Slide No. 4980. [Oecophoridae: Oecophorinae]

neglecta Diakonoff, 1968 (Psorosticha); Bulletin of the U.S. National Museum 257 (1967): 187. Holotype ${ }^{\star}$, Philippine Islands, Luzon, Los Baños, [no date], P. J. Baker. USNM Genitalia Slide No. 89968. Right forewing lacking. [Oecophoridae: Oecophorinae]

negundella Heinrich, 1920 (Gelechia); Proceedings of the U.S. National Museum 57: 63. Holotype $\sigma^{*}$, USA, North Dakota, Barton, r.f. Acer negundo, em. 11 July 1914, M. D. Serdwagen. USNM Type No. 21807, USNM Genitalia Slide No. 8280, USNM Wing Slide No. 8279. [Gelechiidae: Gelechiinae] 
nenia Hodges, 1986 (Dichomeris); Moths of America north of Mexico, fasc. 7.1: 40. Holotype $\diamond^{\star}$, USA, Texas, Bandera Co., ex-larva, 12 May 1966, R. O. \& C. A. Kendall. [Gelechiidae: Dichomeridinae]

neoleuca Clarke, 1971 (Iressa); Smithsonian Contributions to Zoology 56: 159. Holotype ơ, Rapa Island, Haurie, 23 November 1963, J. F. G. \& T. M. Clarke. USNM Type No. 70100, USNM Genitalia Slide No. 26271. Right fore- and hindwing lacking. [Cosmopterigidae: Cosmopteriginae]

neopisoniae Clarke, 1946 (Nealyda); Journal of the Washington Academy of Sciences 36: 427. Holotype ${ }^{*}$, Cuba, Habana Province, Jaimanitas [no date]. USNM Type No. 58212, Genitalia Slide AB Aug. 22, 1934. [Gelechiidae: Gelechiinae]

nestes Busck, 1911 (Stenoma); Proceedings of the U.S. National Museum 40: 214. Holotype $\sigma^{\star}$, French Guiana, St. Jean, Maroni River, [no date], W. Schaus. USNM Type No. 13571, USNM Genitalia Slide No. 13176. [Elachistidae: Stenomatinae]

nienorella Kaila, 1999 (Elachista); Acta Zoologica Fennica 211: 82. Holotype ․, USA, Arizona, Santa Cruz Co., Madera Canyon, Santa Rita Mts., Bog Spring Campground, 5100', 10-26 July 1964, D. Davis. USNM Genitalia Slide No. 90105. [Elachistidae: Elachistinae]

nigra Busck, 1903 (Recurvaria piceaella); Proceedings of the U.S. National Museum 25: 814. Holotype (uncertain sex), USA, New Jersey, Montclair, 22 May, W. D. Kearfott. USNM Type No. 6963. Abdomen lacking. [Gelechiidae: Gelechiinae]

nigra Hodges, 1962 (Periploca); Pan-Pacific Entomologist 38: 94. Holotype o ${ }^{*}$ USA, California, Sacramento Co., Sacramento, r.f. Juniperus sp., 14 February 1961, A. Forbes. USNM Genitalia Slide No. 4367. According to the original description, the type is deposited in the collection of the California Department of Food and Agriculture.

nigrapunctella Busck, 1900 (Cosmopteryx); Proceedings of the U.S. National Museum 23: 235. Holotype $o^{\star}$, USA, Florida, Palm Beach, January 1900, H. Dyar. USNM Type No. 4944, USNM Genitalia Slide No. 3938. [Cosmopterigidae: Cosmopteriginae]

nigricans Busck, 1914 (Athleta); Proceedings of the U.S. National Museum 47: 38. Holotype $\sigma^{*}$, Panama, Trinidad River, June 1912, A. Busck. USNM Type No. 16708, USNM Genitalia Slide No. 13880. [Elachistidae: Stenomatinae] 
nigricornis Clarke, 1986 (Asymphorodes); Smithsonian Contributions to Zoology 416: 214. Holotype ${ }^{\star}$, Marquesas Archipelago, Fatu Hiva, Tahuna, 2000', 27 March 1968, J. F.

G. \& T. M. Clarke. USNM Type No. 100779, USNM Genitalia Slide No. 24843. [Cosmopterigidae: Cosmopteriginae]

nigrimaculella Busck, 1903 (Gelechia); Proceedings of the U.S. National Museum 25: 880. Lectotype $\odot^{\star}$, USA, New York, [no date], Beutenmüller Collection. USNM Type No. 6389; USNM Genitalia Slide No. 8282. Designated by Hodges (1999). [Gelechiidae: Gelechiinae]

nigrinotella Busck, 1908 (Depressaria); Proceedings of the Entomological Society of Washington 9: 88. Lectotype ㅇ, USA, Ohio, Cincinnati, A. F. Braun, 17 June 1905. USNM Type No. 11325, USNM Genitalia Slide No. 328. Left forewing in gelatin capsule. Designated by Hodges (1974). [Elachistidae: Depressariinae]

nigriplaga Dognin, 1904 (Coptotelia); Annales de la Société Entomologique de Belgique 48: 134. Holotype (uncertain sex), Colombia, "Durrant 12.6.12," “6559 Wlsm 1911." Genitalia Slide AB Apr. 14, 1928. [Elachistidae: Depressariinae]

nigrobasiella Clarke, 1932 (Aristotelia); Canadian Entomologist 64: 63. Holotype o*, Canada, British Columbia, Fraser Mills, 6 May 1924, L. E. Marmont. USNM Type No. 43449, USNM Genitalia Slide 5895. [Gelechiidae: Gelechiinae]

niphacma Meyrick, 1916 (Stenoma); Exotic Microlepidoptera 1: 510. Holotype ${ }^{\star}$, French Guiana, St. Jean, Rio Maroni, July 1915, LeM. Genitalia Slide AB Apr. 9, 1934. Although a penciled label on the specimen says "Stenoma chalastis," a red "Type" label affixed to the specimen indicates "This is the TYPE of Stenoma niphacma Meyr., sent to Busck, J.F.G.C. 1949.” [Elachistidae: Stenomatinae]

niveopulvella Chambers, 1875 (Gelechia); Canadian Entomologist 7: 209. Holotype o*, Canada [no other data]. USNM Type No. 9792, USNM Genitalia Slide No. 10679. [Gelechiidae: Gelechiinae]

nonna Clarke, 1986 (Cosmopterix); Smithsonian Contributions to Zoology 416: 309. Holotype \&, Marquesas Archipelago, Hiva Oa, Mt. Feani, 3400', 1 March 1968, J. F. G. \& T. M. Clarke. USNM Type No. 100848, USNM Genitalia Slide No. 25495. [Cosmopterigidae: Cosmopteriginae]

nonstrigella Busck, 1906 (Anacampsis); Canadian Entomologist 38: 121. Holotype $q$, USA, Pennsylvania, Allegheny Co., Oak Station, July. USNM Type No. 9792, USNM Genitalia Slide 10359. [Gelechiidae: Gelechiinae] 
notandella Busck, 1911 (Peleopoda); Proceedings of the U.S. National Museum 40: 209.

510 Holotype $\sigma^{*}$, French Guiana, St. Jean, Maroni, [no date], W. Schaus. USNM Type No. 13562, USNM Genitalia Slide No. 69538. [Peleopodidae]

notandella Busck, 1916 (Gelechia); Proceedings of the Entomological Society of Washington 18: 149. Holotype $\sigma^{*}$, USA, California, San Diego Co., San Diego, [no date], W. S. Wright. USNM Type No. 20071, USNM Genitalia Slide No. 8041. [Gelechiidae: Gelechiinae]

notella Busck, 1914 (Cryptolechia); Proceedings of the U.S. National Museum 47: 30. Holotype ${ }^{*}$, Panama, Trinidad River, June 1912, A. Busck. USNM Type No. 16753, USNM Genitalia Slide No. 69473. The original description erroneously states that the specimen was collected in March. [Elachistidae: Depressariinae]

notolatra Wu, 1998 (Hygroplasta); Zoological Studies 37 (3): 199. Holotype ơ, Malaysia, Sabah, Kinabalu National Park Headquarters area, 1560 m, 8 September 1983, G. Hevel \& W. Steiner. USNM Genitalia Slide No. 89129. [Lecithoceridae]

notolepis Park, 2000 (Dinochares); Zoological Studies 39: 369. Holotype $\sigma^{\star}$, Taiwan, Nantou Co., Rengwati (Lienhauchih), 24 March 1943, S. Issiki. USNM Genitalia Slide No. 82497. [Lecithoceridae]

novimundi Busck, 1915 (Symmoca); Proceedings of the Entomological Society of Washington 17: 84. Holotype ot, USA, Pennsylvania, Roxborough, 21 November 1911, F. Haimbach. USNM Type No. 19230, USNM Genitalia Slide 10661. [Autostichidae: Symmocinae]

nubila Kaila, 1997 (Elachista); Acta Zoologica Fennica 206: 27. Holotype o , USA, Arizona, Pima Co., Santa Rita Mts., Madera Canyon, 4800', 31 July 1959, R. W. Hodges. USNM Genitalia Slide No. 89299. [Elachistidae: Elachistinae]

nubis Hodges, 1999 (Chionodes); Moths of America north of Mexico, fasc. 7.6: 156. Holotype $\sigma^{\star}$, USA, Arizona, Coconino Co., Hart Prairie, 10 mi NNW Flagstaff, 30 June 1961, R. W. Hodges. [Gelechiidae: Gelechiinae]

nuciferae Clarke, 1986 (Asymphorodes); Smithsonian Contributions to Zoology 416: 257. Holotype $\sigma^{*}$, Marquesas Archipelago, Hiva Oa, Atuona, r.f. Cocos nucifera, em. 21 March 1968, J. F. G. \& T. M. Clarke. USNM Type No. 100812, USNM Genitalia Slide No. 24645. [Cosmopterigidae: Cosmopteriginae] 
nuciferae Hodges, 1966 (Batrachedra); Transactions of the American Entomological Society 92: 627. Holotype 9 , Brazil, Bahia, r.f. flowers of Cocos nucifera, [no date], Bondar. USNM Type No. 67874, USNM Genitalia Slide No. 5144. Slide only, no pinned adult found. [Batrachedridae]

nucula Kaila, 1997 (Elachista); Acta Zoologica Fennica 206: 39. Holotype $\sigma^{\star}$, USA, Colorado, Alamosa Co., Zapata Ranch, 7900', ponderosa pine-pinyon pine, cottonwood-aspen, 26 June 1982, R.W. Hodges. USNM Genitalia Slide No. 89280. [Elachistidae: Elachistinae]

obidenna Clarke, 1947 (Filatima); Journal of the Washington Academy of Sciences 37: 274. Holotype $\sigma^{*}$, USA, Arizona, Cochise Co., Paradise, 1-7 April. USNM Type No. 58252, USNM Genitalia Slide No. 9596. [Gelechiidae: Gelechiinae]

obligata Busck, 1914 (Telphusa); Proceedings of the U.S. National Museum 47: 15. Holotype + , Panama, La Chorrera, 12 May, A. Busck. USNM Type No. 15829. [Gelechiidae: Gelechiinae]

obliquistriga Dognin, 1905 (Hypercallia); Annales de la Société Entomologique de Belgique 49: 89. Holotype ${ }^{\star}$, Ecuador, environs de Loja, 1889. USNM Type No. 32361, USNM Genitalia Slide No. 4083. [Oecophoridae: Oecophorinae]

obnubila Busck, 1914 (Catarata); Proceedings of the U.S. National Museum 47: 36. Holotype ơ, Panama, Trinidad River, March 1912, A. Busck. USNM Type No. 16705, USNM Genitalia Slide No. 14085. [Elachistidae: Stenomatinae]

obscurella Beutenmüller, 1888 (Psecadia); Entomologica Americana 4: 29. Holotype $\sigma^{*}$, USA, California, Havilah, [no date], Beutenmüller. USNM Type No. 437. [Elachistidae: Ethmiinae]

obscuromaculella Chambers, 1878 (Cryptolechia); Bulletin of the U.S. Geological and

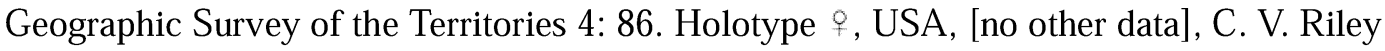
Collection. USNM Type No. 432. [Oecophoridae: Oecophorinae]

obscurosuffusella Chambers, 1874 (Gelechia); Canadian Entomologist 6: 236. Holotype (sex uncertain), USA, Texas [no date]. USNM Type No. 456. Abdomen and hindwings lacking. [Gelechiidae: Gelechiinae]

occidalpella Jäckh, 1978 (Scythris); Bolletino del Museo Civico di Storia Naturali 5: 5. Holotype ${ }^{*}$, Italy, Sestriere, Sopra, 2000 m, 20 August 1933, Rocca \& Della-Beffa. 
USNM Genitalia Slide No. 87773. According to the original description, deposited in Museo Civico Storia Naturale, Genova, Italy. [Xyloryctidae: Scythridinae]

occidentalis Adamski, 1989 (Mastema); Mississippi Agricultural Forest Experimental Station Technical Bulletin 165: 22. Holotype ${ }^{*}$, Arizona, Madera Canyon, Santa Rita Mountains, 2 September 1959, R. W. Hodges. USNM Genitalia Slide No. 81472. [Coleophoridae: Blastobasinae]

occidua Hodges \& Adamski, 1997 (Filatima); Journal of the Lepidopterists' Society 51: 42. Holotype + , USA, Washington, Pullman, r.f. Lupinus ornatus, 3 Aug 1932, J. F. G. Clarke. USNM Genitalia Slide No. 9716. [Gelechiidae: Gelechiinae]

ocellata Busck, 1914 (Catarata); Proceedings of the U.S. National Museum 47: 37. Holotype ${ }^{*}$, Panama, La Chorrera, May 1912, A. Busck. USNM Type No. 16707, W. D. Duckworth Genitalia Slide No. 2567. [Elachistidae: Stenomatinae]

ocellea Forbes, 1930 (Mothonica); Scientific Survey of Porto Rico and the Virgin Islands 12: 131. Holotype $\sigma^{\star}$, Guatemala, Cayuga, March, Schaus \& Barnes. Genitalia Slide AB Sept. 11, 1929. [Elachistidae: Stenomatinae]

ochlodes Walsingham, 1912 (Stenoma); Biologia Centrali-Americana, Lepidoptera Heterocera 4: 166. Holotype 9 , Panama, Canal Zone, Tabernilla, June 1907, A. Busck. Abdomen lacking. [Elachistidae: Stenomatinae]

ochreata Park \& Hodges, 1995 (Dichomeris); Insecta Koreana 12: 32. Holotype o*, Taiwan, Nantou Co., Mei-feng, 30 km S Tayuling, 2200 m, 1-8 June 1980, D. Davis. USNM Genitalia Slide No. 87359. [Gelechiidae: Dichomeridinae]

ochreocostella Chambers, 1878 (Gelechia); Bulletin of the U.S. Geological and Geographic Survey of the Territories 4: 91. Pseudotype 9 , USA, Texas. USNM Type No. 461. Abdomen lacking. According to Miller and Hodges (1990), the holotype female is in MCZ. [Gelechiidae: Gelechiinae]

ochrifoliata Walsingham, 1911 (Telphusa); Biologia Centrali-Americana, Lepidoptera Heterocera 4: 56. Holotype $\sigma^{*}$, Mexico, Veracruz, Cordoba, January, F. Knab. [Gelechiidae: Gelechiinae]

ochrocephala Dietz, 1910 (Holcocera); Transactions of the American Entomological Society 36: 31. Lectotype ${ }^{*}$, USA, West Virginia, Aurora, 21 August 1904, O. Heidemann. USNM Genitalia Slide No. 81025. Designated by Adamski \& Hodges (1996). [Coleophoridae: Blastobasinae] 
ochrogramma Clarke, 1986 (Asymphorodes); Smithsonian Contributions to Zoology 416: 281. Holotype ${ }^{\star}$, Marquesas Archipelago, Nuku Hiva, Tapuaooa, 2500', 30 January 1968,

J. F. G. \& T. M. Clarke. USNM Type No. 100798, USNM Genitalia Slide No. 24753. [Cosmopterigidae: Cosmopteriginae]

ochroleuca Clarke, 1978 (Aniuta); Smithsonian Contributions to Zoology 273: 5. Holotype $\sigma^{\star}$, Chile, Llanquihue, Peulla, 9 March 1959, J. F. G. Clarke. USNM Type No. 73691. [Oecophoridae: Oecophorinae]

oculinota Wu \& Park, 1999 (Timyra); Korean Journal of Systematic Zoology 15: 212. Holotype $\diamond^{*}$ Sri Lanka, Kan. District, Ugalawatta, 13-14 October 1976, Hevel, Dietz, Karunaratne \& Balasooriya. USNM Genitalia Slide No. 88852. [Lecithoceridae]

offula Hodges, 1986 (Dichomeris); Moths of America north of Mexico, fasc. 7.1: 117. Holotype $\sigma^{\star}$, USA, New York, Ithaca, 19 June 1937, J. G. Franclemont. USNM Genitalia Slide No. 9280. [Gelechiidae: Dichomeridinae]

oligoria Clarke, 1986 (Herlinda); Smithsonian Contributions to Zoology 416: 286. Holotype $\sigma^{*}$, Marquesas Archipelago, Hiva Oa, Atuona, 24 February 1968, J. F. G. \& T. M. Clarke. USNM Type No. 100824, USNM Genitalia Slide No. 25106. [Cosmopterigidae: Cosmopteriginae]

onyxijuxta Wu \& Park, 1998 (Hygroplasta); Insecta Koreana 15: 9. Holotype ${ }^{*}$, Sri Lanka, Gal. District, Udugama, Kanneliya Jungle, 400', 6-12 October, 1973, Krombein, Karunarata, Fernando \& Ferdinando. USNM Genitalia Slide No. 88940. [Lecithoceridae]

opinatrix Hodges, 1969 (Periploca); Smithsonian Contributions to Zoology 18: 4. Holotype $o^{*}$, USA, Wyoming, 6 mi NW Newcastle, 13 July 1965, R. W. Hodges. USNM Type No. 69780, USNM Genitalia Slide No. 4292. [Cosmopterigidae: Chrysopeleiinae]

optio Hodges, 1999 (Chionodes); Moths of America north of Mexico, fasc. 7.6: 154. Holotype ${ }^{*}$, USA, Texas, Jeff Davis Co., Davis Mountains, Mount Locke, 6700', 21 October 1973, A. \& M. E. Blanchard. [Gelechiidae: Gelechiinae]

oregonensis Clarke, 1941 (Agonopterix); Proceedings of the U.S. National Museum 90: 65. Holotype $\propto$, USA, Oregon, Salem, March, E. Y. Lansing. USNM Type No. 52079. Specimen not found. [Elachistidae: Depressariinae]

orestella Busck, 1908 (Elachista); Canadian Entomologist 40: 196. Holotype o*, USA, Pennsylvania, Allegheny Co., Oak Station, 11 June 1987, F. Marloff. USNM Type No. 11563. Abdomen lacking. [Elachistidae: Elachistinae] 
orientis Park \& Hodges, 1995 (Dichomeris); Insecta Koreana 12: 36. Holotype ${ }^{*}$, Taiwan, 510 Kaohsiung Co., 10-11 km NE Chiahsien, ca. 300 m, 3-8 July 1980, D. Davis. USNM Genitalia Slide No. 87333. [Gelechiidae: Dichomeridinae]

orion Busck, 1920 (Stenoma); Insecutor Inscitiae Menstruus 8: 90. Holotype ox, Guatemala, Cayuga, [no date], W. Schaus. USNM Type No. 22309. [Elachistidae: Stenomatinae]

orladelaneae Adamski \& Brown, 2002 (Blastobasis); Insecta Koreana 19: 144. Holotype $\uparrow$, New Caledonia, 2 km SW Tinip radio tower, 200 m, 18-19 February 1984, M. Pogue \& M. Epstein. USNM Genitalia Slide No. 81460. [Coleophoridae: Blastobasinae]

ornata Busck, 1934 (Ethmia); Entomologica Americana 13: 168. Holotype ${ }^{*}$, Cuba, [no date], W. Schaus. USNM Type No. 44140, Genitalia Slide AB Apr. 12, 1932. [Elachistidae: Ethmiinae]

oronella Walsingham, 1882 (Gelechia); Transactions of the American Entomological Society 10: 179. Syntype ( $\$$ ), USA, Maine, Orono, [no date], Fernald Collection. Genitalia Slide AB Aug. 6, 1933. According to the original description, described from two specimens. [Gelechiidae: Gelechiinae]

orthogonia Wu, 2000 (Halolaguna); Acta Zootaxonomica Sinica 25: 427. Holotype $\sigma^{*}$, Sarawak, Semengoh Forest Reserve, 15 mi S Kuching, 16 September 1966. USNM Genitalia Slide No. 89123. [Lecithoceridae]

oterosella Busck, 1934 (Ethmia); Entomologica Americana 13: 165. Holotype \&, Cuba, Santiago de las Vegas, Estacion Experimental Agronimica, "leaf-tier on bejuco de canastas" (= Trichostigma octandrum). USNM Type No. 44139, Genitalia Slide AB Apr. 8, 1932. [Elachistidae: Ethmiinae]

oxybela Clarke, 1971 (Gonionota); Smithsonian Contributions to Zoology 95: 8. Holotype ๙, Peru, Cuzco Province [no date]. USNM Type No. 71086. [Oecophoridae: Oecophorinae]

oxygonia Wu \& Park, 1999 (Kalocyrma); Insecta Koreana 16: 136. Holotype ־, Sri Lanka, Kan. District, Kandy, 1800', Peak View Motel, 7-14 January 1970, D. Davis \& B. Rowe. USNM Genitalia Slide No. 89008. [Lecithoceridae]

pacator Hodges, 1999 (Chionodes); Moths of America north of Mexico, fasc. 7.6: 82. Holotype ${ }^{*}$, USA, California, Los Angeles Co., San Gabriel Mountains, Mount Lowe, 9 July 1924. USNM Genitalia Slide No. 15359. [Gelechiidae: Gelechiinae] 
paean Hodges, 1999 (Chionodes); Moths of America north of Mexico, fasc. 7.6: 93. Holotype ${ }^{\star}$, USA, California, San Diego Co., Jacumba, 23 July 1935, E. C. Johnston. USNM Genitalia Slide No. 8165. [Gelechiidae: Gelechiinae]

pagella Hodges, 1966 (Lita); Proceedings of the U.S. National Museum 119: 21. Holotype o*, USA, Arizona, Coconino Co., Fort Valley, 7.5 mi NW Flagstaff, 7350', 27 August 1961, R. W. Hodges. USNM Genitalia Slide No. 10649. [Gelechiidae: Gelechiinae]

pallidastrigella Chambers, 1874 (Gelechia); Canadian Entomologist 6: 244. Syntype (uncertain sex), USA, Texas, [probably Waco], [no date], Belfrage. There are two syntypes, one in USNM and one in MCZ. Specimen not found. [Gelechiidae: Gelechiinae]

pallidella Busck, 1904 (Depressaria); Proceedings of the U.S. National Museum 27: 765. Holotype $\sigma^{*}$, Canada, British Columbia, Kaslo, 1902, H. Dyar \& Cockle. USNM Type No. 7818, USNM Genitalia Slide No. 179. [Elachistidae: Depressariinae]

pallidochrella Chambers, 1873 (Helice); Canadian Entomologist 5: 188. Pseudotype $\sigma^{*}$ USA, Kentucky [no date]. USNM Type No. 494, USNM Genitalia Slide No. 10682. The holotype, deposited in MCZ (Miller and Hodges 1990), is not conspecific with the USNM pseudotype. [Gelechiidae: Gelechiinae]

pallor Hodges, 1999 (Chionodes); Moths of America north of Mexico, fasc. 7.6: 197. Holotype $\sigma^{*}$, USA, Arizona, Coconino Co., Fort Valley, $7.5 \mathrm{mi}$ NW Flagstaff, 28 June 1961, R. W. Hodges. [Gelechiidae: Gelechiinae]

pallulella Busck, 1914 (Stenoma); Proceedings of the U.S. National Museum 47: 47. Holotype $\diamond^{\star}$, Panama, Trinidad River, March 1912, A. Busck. USNM Type No. 16727, USNM Genitalia Slide No. 12866. [Elachistidae: Stenomatinae]

palousella Clarke, 1941 (Depressaria); Proceedings of the U.S. National Museum 90: 171. Holotype 9 , USA, Washington, Pullman, 26 June 1930, J. F. G. Clarke. USNM Type No. 52074, USNM Genitalia Slide No. 502. [Elachistidae: Depressariinae]

palpigera Busck, 1914 (Paraspistis); Proceedings of the U.S. National Museum 47: 10. Syntype (uncertain sex), British West Indies, [no date], F. W. Urich. According to the original description, there is a second syntype in BMNH. [Gelechiidae: Gelechiinae]

paltodoriella Busck, 1903 (Anacampsis); Proceedings of the U.S. National Museum 25: 848. Holotype $\sigma^{*}$, USA, New Mexico, Mesilla Park [no dtae]. USNM Type No. 6372, USNM Genitalia Slide No. 10655. [Gelechiidae: Gelechiinae] 
panella Busck, 1903 (Gelechia); Proceedings of the U.S. National Museum 25: 889. Syntypes $\left(1 \sigma^{*}, 1^{\circ}\right)$, USA, Arizona, Catalina Springs, 10 May $1898\left({ }^{\star}\right)$; California, Los Angeles Co, March (\%). USNM Type No. 6291, USNM Genitalia Slide Nos. 6789, 6791. [Gelechiidae: Gelechiinae]

panurgella Heinrich, 1920 (Holcocera); Proceedings of the U.S. National Museum 57: 71. Holotype 9 , USA, Arizona, Santa Catalina Mountains, r.f. Pinus cembroides, 16-17 June, G. Hofer. USNM Type No. 21812, USNM Genitalia Slide No. 81026. [Coleophoridae: Blastobasinae]

paralagneia Clarke, 1978 (Doina); Smithsonian Contributions to Zoology 273: 21. Holotype $\sigma^{\star}$, Chile, Centro-Austral, January-March 1898, V. Izquerdo. USNM Type No. 73700, USNM Genitalia Slide No. 24122. [Amphisbatidae]

paralevirola Park, 1999 (Lecithocera); Zoological Studies 38: 245. Holotype ${ }^{\star}$, Taiwan, Taichung Co., Baibara (Meiyuan), 24 March 1942, S. Issiki. USNM Genitalia Slide No. 82496. [Lecithoceridae]

paralogella Busck, 1916 (Gelechia); Proceedings of the Entomological Society of Washington 18: 149. Holotype ${ }^{\star}$, USA, San Diego Co., San Diego, W. S. Wright. USNM Type No. 20070, USNM Genitalia Slide No. 7700. [Gelechiidae: Gelechiinae]

paraplutella Busck, 1909 (Gelechia); Proceedings of the Entomological Society of Washington 11: 181. Holotype $\sigma^{\star}$, USA, California, San Diego Co., San Diego, May-July, L. E. Ricksecker. USNM Type No. 12688, USNM Genitalia Slide No. 9938. [Gelechiidae: Gelechiinae]

paraporia Clarke, 1986 (Asymphorodes); Smithsonian Contributions to Zoology 416: 252. Holotype $\$$, Marquesas Archipelago, Nuku Hiva, Tunoa Ridge, 2900', 23 January 1968, J. F. G. \& T. M. Clarke. USNM Type No. 100809, USNM Genitalia Slide No. 25004. [Cosmopterigidae: Cosmopteriginae]

paravexillata Clarke, 1971 (Gonionota); Smithsonian Contributions to Zoology 95: 23. Holotype $\sigma^{\star}$, Venezuela, Aragua, Rancho Grande, 1100 m, 21-25 January 1966, S. S. \& W. D. Duckworth. USNM Type No. 71096. [Oecophoridae: Oecophorinae]

pardella Busck, 1912 (Moriloma); Smithsonian Miscellaneous Collections 59: 3. Holotype + , Panama, Porto Bello, r.f. Conostgia sp. (=Cryptophysa) [no date]. USNM Type No. 14530. [Cosmopterigidae: Cosmopteriginae] 
parephoria Clarke, 1951 (Aristotelia); Journal of the Washington Academy of Sciences 41: 140. Holotype ${ }^{\star}$, Argentina, Tucumán, August 1950, J. Pastrana. USNM Type No. 60943, J. G. F. Clarke Genitalia Slide No. 2858. [Gelechiidae: Gelechiinae]

parilis Hodges, 1969 (Perimede); Smithsonian Contributions to Zoology 18: 12. Holotype ๔, USA, Arkansas, Washington Co., Devil's Den State Park, 10 July 1966, R. W. Hodges. USNM Type No. 69787, USNM Genitalia Slide No. 4802. [Cosmopterigidae: Chrysopeleiinae]

paritor D. Farr \& O. Flint (Batrachedra); Transactions of the American Entomological Society 92: 631. Holotype o*, Jamaica, St. Andrews Parish, Hardware Gap, Dicks Pond Trail, 25 July 1962, O. Farr \& R. Flint. USNM Type No. 67865, USNM Genitalia Slide No. 5168. Left fore- and hindwing lacking. [Batrachedridae]

particornella Busck, 1919 (Perimede); Proceedings of the Entomological Society of Washington 11: 96. Holotype + , USA, Maryland, Montgomery Co., Plummers Island, Potomac River, [no date], USNM Type No. 12269, USNM Genitalia Slide No. 4425. [Cosmopterigidae: Chrysopeleiinae]

parvipulvella Chambers, 1875 (Elachista); Canadian Entomologist 7: 56. Holotype ox, USA, Texas, [no date], Belfrage. USNM Type No. 499. Abdomen lacking. [Elachistidae: Elachistinae]

passerella Busck, 1909 (Psacaphora); Proceedings of the Entomological Society of Washington 11: 95. Holotype $\sigma^{\star}$, USA, Connecticut, East River, 20 July 190?, C. R. Ely. USNM Type No. 12266, USNM Genitalia Slide No. 4992. [Coleophoridae: Momphinae]

passiflorae Clarke, 1982 (Odonna); Journal of Research on the Lepidoptera 20: 47. Holotype 9 , Colombia, Valle, Tenerife, r.f. Passiflora mollissima, June 1981, Chacon \& Rojas. USNM Type No. 100175, USNM Genitalia Slide No. 25051. [Oecophoridae: Oecophorinae]

pastor Hodges, 1999 (Chionodes); Moths of America north of Mexico, fasc. 7.6: 155. Holotype $\sigma^{*}$, USA, Utah, Sanpete Co., Great Basin Experiment Station, near Ephraim, 8850', 14 August 1981, R. W. Hodges. [Gelechiidae: Gelechiinae]

paulella Busck, 1903 (Gelechia); Proceedings of the U.S. National Museum 25: 865. Holotype ${ }^{\star}$, USA, Colorado [no date]. USNM Type No. 6379, USNM Genitalia Slide No. 9946 [Gelechiidae: Gelechiinae] 
pavor Hodges, 1999 (Chionodes); Moths of America north of Mexico, fasc. 7.6: 80. Holo510 type $\%$, USA, California, San Bernardino Co., San Bernardino Mountain, Camp Baldy, 16-23 July. USNM Genitalia Slide No. 15155. [Gelechiidae: Gelechiinae]

pecosella Busck, 1907 (Mompha); Proceedings of the Entomological Society of Washington 8: 96. Holotype ${ }^{*}$, USA, New Mexico, Pecos, June, T. D. A. Cockerell. USNM Type No. 9901. [Coleophoridae: Momphinae]

pecten Clarke, 1951 (Coptotelia); Acta Zoologica Lilloana 11: 342. Holotype $\%$, Guatemala, Volcan Santa Maria, June, W. Schaus \& Barnes. USNM Type No. 61116, USNM Genitalia Slide No. 69412. [Oecophoridae: Oecophorinae]

peculella Busck, 1914 (Anacampsis); Proceedings of the U.S. National Museum 47: 7. Syntype (\%), Panama, Paraiso, February-May, A. Busck. Genitalia Slide AB Oct. 12, 1937. [Gelechiidae: Gelechiinae]

pelodes Walsingham, 1913 (Stenoma); Biologia Centrali-Americana, Lepidoptera Heterocera 4: 180. Holotype \%, Panama, Canal Zone, Tabernilla, June 1907, A. Busck. USNM Genitalia Slide No. 14047. [Elachistidae: Stenomatinae]

pelta Hodges, 1986 (Dichomeris); Moths of America north of Mexico, fasc. 7.1: 99. Holotype + , USA, South Carolina, Charleston Co., McClellanville, Wedge Plantation, 28 April 1981, R. W. Hodges. Abdomen lacking. [Gelechiidae: Dichomeridinae]

pennella Busck, 1907 (Paltodora); Proceedings of the Entomological Society of Washington 8: 88. Holotype + , USA, Arizona, Bright Angel [no date]. USNM Type No. 9891, USNM Genitalia Slide No. 5602. [Gelechiidae: Gelechiinae]

pensor Hodges, 1966 (Chedra); Transactions of the American Entomological Society 92: 638. Holotype $o^{\star}$, USA, Arizona, Coconino Co., West Fork, 16 mi SW Flagstaff, 4 July 1961, R. W. Hodges. USNM Type No. 67869. [Batrachedridae]

penthica Walsingham, 1912 (Ethmia); Biologia Centrali-Americana, Lepidoptera Heterocera 4: 146. Holotype $\sigma^{\star}$, Mexico, Oaxaca [no date]. USNM Type No. 68204, USNM Genitalia Slide No. 98455. [Elachistidae: Ethmiinae]

perceptella Busck, 1914 (Dichomeris); Proceedings of the U.S. National Museum 47: 19. Holotype $o^{\star}$, Panama, La Chorrera, May 1912, A. Busck. USNM Type No. 16675, Genitalia Slide AB Sept. 17, 1928. [Gelechiidae: Dichomeridinae] 
percnophthalma Clarke, 1971 (Stathmopoda); Smithsonian Contributions to Zoology 56: 178. Holotype ơ, Rapa Island, Maugaoa, 950', 23 October 1963, J. F. G. \& T. M. Clarke. USNM Type No. 70117. [Oecophoridae: Stathmopodinae]

peregrina Clarke, 1971 (Palintropa); Smithsonian Contributions to Zoology 56: 138. Holotype $\sigma^{*}$, Rapa Island, Maura, 600', 16 September 1963, J. F. G. \& T. M. Clarke. USNM Type No. 70095, USNM Genitalia Slide No. 26266. Right fore- and hindwing lacking. [Gelechiidae: Gelechiinae]

pereyra Clarke, 1947 (Chionodes); Journal of the Washington Academy of Sciences 37: 253. Holotype ${ }^{*}$, USA, Florida, Indian River Co., Vero Beach, April-May 1941, J. R. Malloch. USNM Type No. 58243, USNM Genitalia Slide No. 8304. [Gelechiidae: Gelechiinae]

pergandeella Busck, 1908 (Depressaria); Proceedings of the Entomological Society of Washington 9: 89. Holotype o*, USA, Nebraska, [no date], T. Pergande. USNM Type No. 11327, USNM Genitalia Slide No. 280. [Elachistidae: Depressariinae]

pergandeella Busck, 1901 (Triclonella); Journal of the New York Entomological Society 8: 237. Lectotype ${ }^{\star}$, USA, District of Columbia, em. 12 August 1898. USNM Type No. 5354, USNM Genitalia Slide No. 3994. Designated by Hodges (1978). [Cosmopterigidae: Cosmopteriginae]

periculella Busck, 1910 (Gelechia); Proceedings of the Entomological Society of Washington 11: 178. Holotype ${ }^{*}$, USA, California, Humbolt Co., Bair's Ranch, Redwood Creek, July, H. S. Barber. USNM Type No. 12698. Abdomen lacking. [Gelechiidae: Gelechiinae]

periphereia Clarke, 1964 (Gonionota); Proceedings of the U.S. National Museum 115: 77. Holotype $\diamond^{\star}$, Ecuador, environs de Loja, Dognin Collection. USNM Type No. 66377, USNM Genitalia Slide No. 68961. [Oecophoridae: Oecophorinae]

perissa Diakonoff, 1968 (Odites); Bulletin of the U.S. National Museum 257 (1967): 121. Holotype $\sigma^{*}$, Philippine Islands, Luzon, Mt. Makiling, [no date], P. J. Baker. USNM Genitalia Slide No. 89989. [Lecithoceridae]

perniva Kaila, 1997 (Elachista); Acta Zoologica Fennica 206: 47. Holotype ${ }^{\star}$, USA, Wyoming, 6 mi NW Newcastle, 5 July 1965, R. W. Hodges. USNM Genitalia Slide No. 89286. [Elachistidae: Elachistinae] 
pernivis Diakonoff, 1968 (Metathrinca); Bulletin of the U.S. National Museum 257 (1967): 118. Holotype ${ }^{*}$, Philippine Islands, Mindao Province, Maco, Tagum, sea level, [no date], H. Hoogstraal. USNM Genitalia Slide No. 68995. [Xyloryctidae: Xyloryctinae]

peronia Busck, 1913 (Stenoma); Insecutor Inscitiae Menstruus 1: 90. Holotype ${ }^{\star}$, British Guiana, Belair, on bird vine (Hasanthus sp.) [no date]. USNM Type No. 16014. Abdomen lacking. [Elachistidae: Stenomatinae]

perpensa Clarke, 1947 (Filatima); Journal of the Washington Academy of Sciences 37: 268. Holotype ${ }^{\star}$, USA, California, Somona Co., The Geysers, 21 June 1938, E. C. Johnston. USNM Type No. 58248, USNM Genitalia Slide No. 9605. [Gelechiidae: Gelechiinae]

perplexa Clarke, 1951 (Aristotelia); Journal of the Washington Academy of Sciences 41: 140. Holotype $\sigma^{*}$, Argentina, Tigre, March-April 1939, F. Bourquin. USNM Type No. 60942, USNM Genitalia Slide No. 9605. [Gelechiidae: Gelechiinae]

perpulchra Walsingham, 1912 (Ethmia); Biologia Centrali-Americana, Lepidoptera Heterocera 4: 146. Holotype + , Mexico, Veracruz, Orizaba, [no date], W. Schaus. USNM Type No. 68205. [Elachistidae: Ethmiinae]

perseaphaga Clarke, 1951 (Coptotelia); Acta Zoologica Lilloana 11: 345. Holotype $\sigma^{*}$, Costa Rica, San Pedro Montes de Oca, r.f. avocado (Persea sp.), May 1932, C. H. Ballou. USNM Type No. 61113, USNM Genitalia Slide No. 69414. [Oecophoridae: Oecophorinae]

perspicillella Walsingham, 1888 (Butalis); Insect Life 1: 114. Holotype ( $\left.{ }^{x}\right)$, USA, California, Folsom, 15 July 1885. USNM Genitalia Slide No. 15847. [Xyloryctidae: Scythridinae]

peruviella Busck, 1911 (Filinota); Proceedings of the U.S. National Museum 40: 207. Holotype (uncertain sex), Peru, [no date], W. Schaus. USNM Type No. 13560, USNM Genitalia Slide No. 69327 (integumen only). [Oecophoridae: Oecophorinae]

pervada Clarke, 1962 (Gnorimoschema); Entomological News 23: 101. Holotype o*, Japan, Kyûsyû, Usuki, Ôita, em. 28 August 1956, r.f. Solanum lyratum, Someya. USNM Type No. 65832, J. F. G. Clarke Genitalia Slide No. 10616. Specimen not found. [Gelechiidae: Gelechiinae] 
petalumensis Clarke, 1947 (Chionodes); Journal of the Washington Academy of Sciences 37: 250. Holotype ${ }^{\star}$, USA, California, Sonoma Co., Petaluma, 29 August 1939, E. C. Johnston. USNM Type No. 58241, USNM Genitalia Slide No. 8325. [Gelechiidae: Gelechiinae]

petrella Busck, 1915 (Gnorimoschema); Proceedings of the Entomological Society of Washington 17: 83. Holotype ${ }^{\star}$, USA, New Hampshire, Hampton, May, S. A. Shaw. USNM Type No. 19228, USNM Genitalia Slide No. 7239. [Gelechiidae: Gelechiinae]

pexa Hodges, 1969 (Sorhagenia); Smithsonian Contributions to Zoology 18: 9. Holotype ơ, USA, Arkansas, Washington Co., Devil's Den State Park, 20 June 1966, R. W. Hodges. USNM Type No. 69785, USNM Genitalia Slide No. 4773. [Cosmopterigidae: Chrysopeleiinae]

phaeobregma Clarke, 1978 (Doina); Smithsonian Contributions to Zoology 273: 30. Holotype ${ }^{\star}$, Chile, Centro-Austral, January-March 1898, V. Izquerdo. USNM Type No. 73708, USNM Genitalia Slide No. 24261. [Amphisbatidae]

phaeodelta Clarke, 1986 (Asymphorodes); Smithsonian Contributions to Zoology 416: 273. Holotype \&, Marquesas Archipelago, Nuku Hiva, Taiohae, 26 January 1968, J. F. G. \& T. M. Clarke. USNM Type No. 100817, USNM Genitalia Slide No. 24842. [Cosmopterigidae: Cosmopteriginae]

phaeoxantha Clarke, 1986 (Herlinda); Smithsonian Contributions to Zoology 416: 288. Holotype ${ }^{\star}$, Marquesas Archipelago, Hiva Oa, trail to Mt. Feani, 2200', 20 February 1968, J. F. G. \& T. M. Clarke. USNM Type No. 100826, USNM Genitalia Slide No. 25152. [Cosmopterigidae: Cosmopteriginae]

phalacropa Meyrick, 1932 (Stenoma); Exotic Microlepidoptera 4: 303. Holotype ơ, Panama, Taboga Island, June, A. Busck. Genitalia Slide AB May 22, 1934. [Elachistidae: Stenomatinae]

phalarogramma Clarke, 1986 (Asymphorodes); Smithsonian Contributions to Zoology 416: 249. Holotype $\%$, Marquesas Archipelago, Hiva Oa, trail to Mt. Feani, 2000', 20 February 1968, J. F. G. \& T. M. Clarke. USNM Type No. 100807, USNM Genitalia Slide No. 24992. [Cosmopterigidae: Cosmopteriginae]

phillita Clarke, 1973 (Profilinota); Journal of the Lepidopterists' Society 27: 102. Holotype $\sigma^{*}$, Venezuela, Aragua, Rancho Grande, 31 October 1966, S. S. \& W. D. Duckworth. USNM Type No. 72172, USNM Genitalia Slide No. 69532. Right fore- and hindwing lacking. [Oecophoridae: Oecophorinae] 
zootaxA phococara Clarke, 1978 (Dita); Smithsonian Contributions to Zoology 273: 48. Holotype 510 , Chile, Llanquihue, Rio Maullin, 6 January 1966, O. Flint \& Cekalovic. USNM Type No. 73716, USNM Genitalia Slide No. 24210. Right fore- and hindwing lacking. [Oecophoridae: Oecophorinae]

phoebe Busck, 1911 (Stenoma); Proceedings of the U.S. National Museum 40: 222. Holotype ${ }^{*}$, French Guiana, St. Jean, Maroni River, [no date], W. Schaus. USNM Type No. 13586, USNM Genitalia Slide No. 12819. Left fore- and hindwing lacking. [Elachistidae: Stenomatinae]

phthiochroma Clarke, 1971 (Gonionota); Smithsonian Contributions to Zoology 95: 32. Holotype $\sigma^{*}$, Bolivia, Cochabamba, Incachaca, tropical cloud area, 2100 m, 27 August-5 September 1956, L. E. Peña. USNM Type No. 71102, USNM Genitalia Slide No. 68780. [Oecophoridae: Oecophorinae]

physaliella Chambers, 1872 (Gelechia); Canadian Entomologist 4: 173. Syntype + , USA, Kentucky, em. 14 April, r.f. leaf mines on Physalis viscosa. Specimen not found. [Gelechiidae: Gelechiinae]

phytolaccae Clarke, 1946 (Nealyda); Journal of the Washington Academy of Sciences 36: 427. Holotype $\sigma^{*}$, USA, Florida, Bonefish Key, r.f. Phytolacca decandra, 21 March 1945, C. L. Griswold. Specimen not found. [Gelechiidae: Gelechiinae]

phytomiella Busck, 1914 (Anacampsis); Proceedings of the U.S. National Museum 47: 8. Syntypes (25), Panama, Alhajuela, Cabima, and Porto Bello, March-June, A. Busck. USNM Type No. 15820. [Gelechiidae: Gelechiinae]

phytoptera Busck, 1914 (Stenoma); Proceedings of the U.S. National Museum 47: 44. Holotype 9 , Panama, Trinidad River, March 1912, A. Busck. USNM Type No. 16720, W. D. Duckworth Genitalia Slide No. 4058. [Elachistidae: Stenomatinae]

picolella Busck, 1914 (Beltheca); Proceedings of the U.S. National Museum 47: 5. Syntypes ( 2 , 1 uncertain sex), Panama, Cabima, May, [no date], A. Busck. USNM Type No. 16664. Abdomen lacking from one specimen. [Gelechiidae: Gelechiinae]

pinax Hodges, 1999 (Chionodes); Moths of America north of Mexico, fasc. 7.6: 149. Holotype + , USA, Arizona, Coconino Co., Hart Prairie, 10 mi NNW Flagstaff, 8500', 9 September 1961, R. W. Hodges. USNM Genitalia Slide No. 8532. [Gelechiidae: Gelechiinae] 
pinifoliella Chambers, 1880 (Gelechia); Journal of the Cincinnati Society of Natural History 2: 181. Holotype $\sigma^{*}$, USA, Texas [no date]. USNM Type No. 458. Abdomen lacking. [Gelechiidae: Gelechiinae]

piperata Hodges, 1962 (Anoncia); Entomologica Americana 42: 106. Holotype ơ, USA, California, Tulare Co., Smoky Valley, 6200', 8 June 1944, C. Henne. USNM Type No. 66088, USNM Genitalia Slide No. 4051. [Cosmopterigidae: Cosmopteriginae]

piscarifurca Wu \& Park, 1999 (Torodora); Tinea 16 (1): 64. Holotype $\sigma^{*}$, Sri Lanka, Anu. District, Irrigation Bungalow, Padaviya, 180', 27 February-9 March 1970, D. Davis \& B. Rowe. USNM Genitalia Slide No. 10527. [Lecithoceridae]

pisoniae Busck, 1900 (Nealyda); Proceedings of the U.S. National Museum 23: 229. Holotype $q$, USA, Florida, Palm Beach, r.f. Pisonia aculeata, em. February 1900, H. Dyar. [Gelechiidae: Gelechiinae]

placatrix Hodges, 1969 (Aeaea); Smithsonian Contributions to Zoology 18: 8. Holotype १, USA, Arkansas, Washington Co., Devil's Den State Park, 20 June 1966, R. W. Hodges. USNM Type No. 69783, USNM Genitalia Slide No. 4555. [Cosmopterigidae: Chrysopeleiinae]

placendiella Busck, 1908 (Batrachedra); Canadian Entomologist 40: 195. Holotype o , USA, Pennsylvania, Allegheny Co., Oak Station, F. Marloff. USNM Type No. 11561, R. W. Hodges Genitalia Slide No. 723. [Batrachedridae]

platyochra Clarke, 1947 (Filatima); Journal of the Washington Academy of Sciences 37: 272. Holotype ${ }^{\star}$, USA, California, Riverside Co., Dixieland, 15-30 April 1922, O. C. Poling. USNM Type No. 58256, USNM Genitalia Slide No. 9618. [Gelechiidae: Gelechiinae]

plaumanni Powell, 1973 (Ethmia); Smithsonian Contributions to Zoology 120: 183. Holotype $\sigma^{*}$, Brazil, Nova Teutonia, 500 m, $27^{\circ} 11^{\prime} \mathrm{S}, 52^{\circ} 23^{\prime} \mathrm{W}$, September 1961, F. Plaumann. USNM Genitalia Slide No. 98452. [Elachistidae: Ethmiinae]

plautata Wu, 1998 (Tisis); Zoological Studies 37: 195. Holotype ${ }^{\star}$, Indonesia, West Java, Mts. Gede-Panggrango, Tjibodas, 1400 m, 5 October 1966, J. F. G. \& T. M. Clarke. USNM Genitalia Slide No. 86857. [Lecithoceridae]

plemmelia Clarke, 1986 (Asymphorodes); Smithsonian Contributions to Zoology 416: 188. Holotype $\sigma^{\star}$, Marquesas Archipelago, Hiva Oa, 1 March 1968, J. F. G. \& T. M. Clarke. USNM Type No. 100775, USNM Genitalia Slide No. 24995. [Cosmopterigidae: Cosmopteriginae] 
pleurotella Walsingham, 1911 (Aerotypia); Biologia Centrali-Americana, Lepidoptera 510 Heterocera 4: 82. Holotype $\diamond^{\star}$, Mexico, Morelos, Cuernavaca, June 1906, W. Schaus. Abdomen and right fore- and hindwing lacking. [Gelechiidae: Gelechiinae]

plicata Wu \& Park, 1999 (Lecithocera); Insecta Koreana 16: 5. Holotype ox, Sri Lanka, Rat. District, Uggalkaltota, Irrigation Bungalow, 350', 31 January-8 February 1970. D. Davis \& B. Rowe. USNM Genitalia Slide No. 88846. [Lecithoceridae]

plicata Walsingham, 1911 (Ptilostonychia); Biologia Centrali-Americana, Lepidoptera Heterocera 4: 109. Holotype ${ }^{\star}$, Panama, Canal Zone, Tabernilla, June 1907, A. Busck. [Gelechiidae: Gelechiinae]

plumbeella Beutenmüller, 1889 (Psecadia semilugens var.); Entomologica Americana 5: 9. Holotype 9 , USA, Texas, [no date], Beutenmüller. USNM Type No. 438. Abdomen lacking. [Elachistidae: Ethmiinae]

plummerella Busck, 1908 (Agonopterix); Proceedings of the U.S. National Museum 35: 199. Lectotype $\sigma^{\star}$, USA, Maryland, Montgomery Co., Plummers Island, Potomac River, 3 October 1908, H. S. Barber. USNM Type No. 11943. Designated by Hodges (1972). [Elachistidae: Depressariinae]

plumosa Busck, 1914 (Stenoma); Proceedings of the U.S. National Museum 47: 47. Holotype $\sigma^{\star}$, Panama, Trinidad River, March 1912, A. Busck. USNM Type No. 16724, USNM Genitalia Slide No. 17806. [Elachistidae: Stenomatinae]

plutella Chambers, 1874 (Neda); Canadian Entomologist 6: 244. Syntype ơ, USA, Texas, [probably Waco] [no date]. J. F. G. Clarke Genitalia Slide No. 10521. Specimen not found. [Gelechiidae: Gelechiinae]

plutella Chambers, 1875 (Phaetusa); Canadian Entomologist 7: 106. Holotype (uncertain sex), USA, Texas [no date]. R. W. Hodges Genitalia Slide No. 3057. Specimen not found. [Gelechiidae: Gelechiinae]

plutonella Heinrich, 1920 (Tosca); Proceedings of the U.S. National Museum 57: 68. Holotype ${ }^{*}$, USA, New Mexico, Manzano National Forest, Hell Canyon, r.f. Prunus, [no date], C. Heinrich. USNM Type No. 21809, Heinrich Genitalia Slide No. 25 Feb 1918. [Gelechiidae: Gelechiinae]

plutor Hodges, 1999 (Chionodes); Moths of America north of Mexico, fasc. 7.6: 91. Holotype ${ }^{\star}$, USA, Texas, Terrell Co., Sanderson, 25 April 1981, E. C. Knudson. USNM Genitalia Slide No. 448. [Gelechiidae: Gelechiinae] 
poecilia Clarke, 1971 (Gonionota); Smithsonian Contributions to Zoology 95: 31. Holotype $\diamond^{\star}$, Venezuela, Aragua, Rancho Grande, 1100 m, January 1966, S. S. \& W. D. Duckworth. USNM Type No. 71101. [Oecophoridae: Oecophorinae]

poecilosoma Walsingham, 1911 (Zelosyne); Biologia Centrali-Americana, Lepidoptera Heterocera 4: 51. Holotype $\sigma^{*}$, Panama, Canal Zone, Tabernilla, June 1907, A. Busck. [Gelechiidae: Gelechiinae]

pogonikuma Wu \& Park, 1999 (Lecithocera); Insecta Koreana 16: 9. Holotype o*, Sri Lanka, Kan. District, Udawattakele, 2100', 5 October 1976, black light, Hevel, Dietz, Karunaratne \& Balasooriya. USNM Genitalia Slide No. 89001. [Lecithoceridae]

poliopterus Clarke, 1986 (Asymphorodes); Smithsonian Contributions to Zoology 416: 241. Holotype ${ }^{\star}$, Marquesas Archipelago, Hiva Oa, Atuona, 26 February 1968, J. F. G. \& T. M. Clarke. USNM Type No. 100800, USNM Genitalia Slide No. 24856. [Cosmopterigidae: Cosmopteriginae]

pollostella Busck, 1906 (Evippe); Proceedings of the U.S. National Museum 30: 725. Holotype $q$, USA, Texas [no date]. USNM Type No. 9768. Abdomen lacking. [Gelechiidae: Gelechiinae]

ponderosae Hodges \& Stevens, 1978 (Coleotechnites); Journal of the Lepidopterists' Society 32: 118. Holotype $\sigma^{*}$, USA, Colorado, Boulder, July 1971, r.f. Pinus ponderosa, J. Staley. USNM Type No. 75471. [Gelechiidae: Gelechiinae]

popa Hodges, 1999 (Chionodes); Moths of America north of Mexico, fasc. 7.6: 167. Holotype ${ }^{*}$, USA, California, Los Angeles Co., Mint Canyon, 24 January 1942, r.f. Sphaeralcea fasciculata, em. 27 February 1942, C. Henne. [Gelechiidae: Gelechiinae]

porriginosa Hodges, 1962 (Anoncia); Entomologica Americana 42: 102. Holotype o*, USA, California, Claremont, [no date], Metz. USNM Type No. 66086. [Cosmopterigidae: Cosmopteriginae]

portentosa Busck, 1914 (Zetesima); Proceedings of the U.S. National Museum 47: 39. Holotype ${ }^{\star}$, Panama, Porto Bello, March 1911, A. Busck. USNM Type No. 16709, USNM Genitalia Slide No. 14034. [Elachistidae: Stenomatinae]

potosí Busck, 1912 (Metopleura); Proceedings of the Entomological Society of Washington 14: 84. Holotype ․ Mexico, San Luis Potosi, Cerritos, August, A. Busck. USNM Type No. 14523. Left forewing lacking. [Gelechiidae: Gelechiinae] 

Slide No. 15141. [Gelechiidae: Gelechiinae]

praeco Hodges, 1999 (Chionodes); Moths of America north of Mexico, fasc. 7.6: 209. Holotype ${ }^{*}$, USA, Michigan, Presque Isle Co., Ocqueoc Lake, 25-26 July 1970, R. \& E. Hodges. USNM Genitalia Slide No. 5797. [Gelechiidae: Gelechiinae]

praedives Diakonoff, 1968 (Astiarcha); Bulletin of the U.S. National Museum 257 (1967): 169. Holotype + , Philippine Islands, Luzon, Mt. Makiling, [no date], P. J. Baker. USNM Genitalia Slide No. 89980. [Oecophoridae: Oecophorinae]

praetexta Clarke, 1950 (Darlia); Journal of the Washington Academy of Sciences 40: 288. Holotype ${ }^{*}$, Argentina, Tigre, May 1939, F. Bourquin. USNM Type No. 59523, Genitalia Slide AB Sept. 17, 1939. Right fore- and hindwing lacking. [Gelechiidae: Gelechiinae]

praetor Hodges, 1999 (Chionodes); Moths of America north of Mexico, fasc. 7.6: 224. Holotype o*, USA, Utah, Sanpete Co., head Ephraim Canyon, 10,000-10,300', 28 July 1981, R. W. Hodges. [Gelechiidae: Gelechiinae]

prattiella Busck, 1915 (Ethmia); Proceedings of the Entomological Society of Washington 17: 85. Holotype + , USA, Texas, Zavalla Co., Nueces River, [no date], F. C. Pratt. USNM Type No. 19232. [Elachistidae: Ethmiinae]

primella Busck, 1907 (Sophronia); Proceedings of the Entomological Society of Washington 8: 89. Holotype $\sigma^{\star}$, western USA [no other data]. USNM Type No. 9892, USNM Genitalia Slide No. 86516. [Gelechiidae: Gelechiinae]

princeps Busck, 1910 (Gnorimoschema); Proceedings of the Entomological Society of Washington 11: 175. Holotype $\%$, USA, California [no date]. USNM Type No. 12682. Abdomen lacking. [Gelechiidae: Gelechiinae]

priscella Busck, 1912 (Tocasta); Smithsonian Micellaneous Collections 59: 4. Holotype *, Panama, Cabima, May, A. Busck. USNM Type No. 14562. Left forewing lacking. [Elachistidae: Agonoxeninae]

procedes Clarke, 1947 (Filatima); Journal of the Washington Academy of Sciences 37: 263. Holotype $\sigma^{*}$, USA, Texas, Presidio, r.f. Salix exigua, em. 22 October 1943, J. H. Russell. USNM Type No. 58257, USNM Genitalia Slide No. 9620. [Gelechiidae: Gelechiinae] 
procus Hodges, 1999 (Chionodes); Moths of America north of Mexico, fasc. 7.6: 92. Holotype 9 , USA, New Mexico, Socorro Co., Gran Quivira National Monument, 1-3 July 1964, D. R. Davis. USNM Genitalia Slide No. 8161. [Gelechiidae: Gelechiinae]

promyctera Wu \& Park, 1998 (Hygroplasta); Insecta Koreana 15: 9. Holotype o*, Sri Lanka, Kan. District, 5 mi NW Mahiyangana, 30 March-9 April 1971, P. \& P. Spangler. USNM Genitalia Slide No. 89211. [Lecithoceridae]

protensa Park, 1995 (Anarsia); Tropical Lepidoptera 6: 60. Holotype ${ }^{\star}$, Taiwan, Nantou Co., Mei-feng, 30 km S Tayuling, 2200 m, 1-8 June 1980, D. Davis. USNM Genitalia Slide No. 87390. [Gelechiidae: Gelechiinae]

protuberans Clarke, 1950 (Pseudethmia semilugens); Journal of the Washington Academy of Sciences 40: 163. Holotype ${ }^{*}$, USA, California, Imperial Valley, Dixieland, 1-15 March 1922, O. C. Poling. USNM Type No. 59369, USNM Genitalia Slide No. 87686. [Elachistidae: Ethmiinae]

proximella Busck, 1912 (Ethmia); Proceedings of the Entomological Society of Washington 114: 84. Holotype $\%$, Mexico, Tehuacan, 10 June, R. Müller. USNM Type No. 14524. [Elachistidae: Ethmiinae]

psepsa Hodges, 1978 (Anoncia); Moths of America north of Mexico, fasc. 6.1: 65. Holotype 9 , USA, Arizona, White Mountains, 7000', 5-15 July 1925, O. C. Poling. USNM Genitalia Slide No. 9117. [Cosmopterigidae: Cosmopteriginae]

pseudofondella Busck, 1908 (Gelechia); Proceedings of the Entomological Society of Washington 9: 87. Holotype $\odot^{*}$, USA, Pennsylvania, Brighton [no date]. USNM Type No. 11323, USNM Genitalia Slide No. 8321. [Gelechiidae: Gelechiinae]

pseusta Diakonoff, 1968 (Scaeothyris), Bulletin of the U.S. National Museum 257: 164. Holotype $o^{\star}$, Philippine Islands, Mindinao, Davao Province, east slope of Mount McKinley, 3000', 14 September 1946. USNM Genitalia Slide No. 68999. Right fore- and hindwing lacking. [Cosmopterigidae: Cosmopteriginae]

psiloptera Barnes \& Busck, 1920 (Gelechia); Contributions to the Natural History of the Lepidoptera of North America 4: 230. Holotype ${ }^{\star}$, Canada, Quebec, Ottawa Co., Meach Lake [no date]. USNM Genitalia Slide No. 7932. [Gelechiidae: Gelechiinae]

psoraliella Barnes \& Busck, 1920 (Anacampsis); Contributions to the Natural History of the Lepidoptera of North America 4: 226. Holotype $\sigma^{*}$ USA, Iowa, Sioux City, r.f. Psoralea argophylla [no date]. USNM Genitalia Slide No. 10660. [Gelechiidae: Gelechiinae] 
pteleae Barnes \& Busck, 1920 (Agonopteryx); Contributions to the Natural History of the 510 Lepidoptera of North America 4: 231. Holotype 9 , USA, Illinois, Decatur, r.f. Ptelea trifoliata [no date]. USNM Type No. 22666. [Elachistidae: Depressariinae]

pteryxiphaga Clarke, 1952 (Depressaria); Smithsonian Miscellaneous Collections 117: 16. Holotype $\sigma^{*}$, USA, Wyoming, Ten Sleep, r.f. Pteryxia terebinthina calcarea, 7 July 1950, J. F. G. Clarke. USNM Type No. 61124, USNM Genitalia Slide No. 640. [Elachistidae: Depressariinae]

puertella Busck, 1916 (Gelechia); Proceedings of the Entomological Society of Washington 18: 148. Holotype o*, USA, California, San Diego Co., La Puerta [no date]. USNM Type No. 20062, USNM Genitalia Slide No. 7571. [Gelechiidae: Gelechiinae]

pumilis Busck, 1914 (Catarata); Proceedings of the U.S. National Museum 47: 36. Holotype $o^{*}$, Panama, Trinidad River, March 1912, A. Busck. USNM Type No. 16704, USNM Genitalia Slide No. 12813. [Elachistidae: Stenomatinae]

punctessa Powell, 1973 (Ethmia); Smithsonian Contributions to Zoology 120: 213. Holotype $\sigma^{*}$, Mexico, Nueva Leon, 3 mi E Galeana, 7-9 August 1963, W. D. Duckworth \& D. Davis. USNM Genitalia Slide No. 98451. [Elachistidae: Ethmiinae]

punctiferella Busck, 1906 (Mompha); Proceedings of the U.S. National Museum 30: 731. Holotype ${ }^{x}$, Texas, Victoria [no date]. USNM Type No. 9773, R. W. Hodges Genitalia Slide No. 2040. [Cosmopterigidae: Chrysopeleiinae]

purpureofusca Walsingham, 1882 (Gelechia); Transactions of the American Entomological Society 10: 184. Lectotype ․, USA, Maine, Orono, 23 June 1881. Designated by Hodges (1986). [Gelechiidae: Dichomeridinae]

purpuriella Busck, 1909 (Psacaphora); Proceedings of the Entomological Society of Washington 11: 96. Holotype $q$, USA, Colorado, Florissant, r.f. flowers of Argentina anserina, 22 June 1907, T. D. A. Cockerell,. USNM Type No. 12268, USNM Genitalia Slide No. 5508. Left fore- and hindwing lacking. [Coleophoridae: Momphinae]

purpuriella Chambers, 1874 (Chrysopeleia); Canadian Entomologist 6: 73. Syntypes (1 $1{ }^{\star}$, 1 ㅇ), USA, Kentucky [no date]. USNM Type No. 501. Both specimens lacking abdomen. [Gelechiidae: Chrysopeleiinae]

putella Busck, 1914 (Recurvaria); Proceedings of the U.S. National Museum 47: 11. Syntype + , Panama, Paraiso, Isthmian Canal Zone, La Chorrera, April-May, A. Busck. USNM Type No. 15824, USNM Genitalia Slide No. 10665. [Gelechiidae: Gelechiinae] 
pygmaea Busck, 1912 (Urangela); Smithsonian Miscellaneous Collections 59: 2. Holotype ${ }^{\star}$, Panama, Porto Bello, March. USNM Type No. 14529. [Cosmopterigidae: Cosmopteriginae]

quadristrigella Busck, 1913 (Coleophora); Journal of Entomology and Zoology (Pomona College) 5: 96. Syntypes (4), USA, California, San Diego, June-July, W. S. Wright. USNM Type No. 15607. Specimens not found. [Coleophoridae: Coleophorinae]

quaintancella Dietz, 1910 (Valentinia); Transactions of the American Entomological Society 36: 15. Lectotype ${ }^{*}$, USA, Quaintance No. 858, 20 November 1907. USNM Genitalia Slide No. 11293. Designated by Adamski \& Hodges (1996). [Coleophoridae: Blastobasinae]

querciella Chambers, 1872 (Gelechia); Canadian Entomologist 4: 223. Holotype 9 , USA, Kentucky [no date]. USNM Type No. 460. Abdomen lacking. According to Hodges (1986), the type is lost; he further indicates that a pseudotype is in MCZ. [Gelechiidae: Gelechiinae]

querciella Busck, 1908 (Stenoma); Proceedings of the Entomological Society of Washington 10: 112. Holotype + , USA, Pennsylvania, Hazleton, r.f. Quercus, August 1903, W. G. Dietz. USNM Type No. 11946, USNM Genitalia Slide No. 98109. [Elachistidae: Stenomatinae]

quinquepunctella Busck, 1903 (Aristotelia); Proceedings of the U.S. National Museum 25: 804. Holotype + , USA, Pennsylvania, June. USNM Type No. 6353, USNM Genitalia Slide No. 5911. Forewings and right hindwing lacking. [Gelechiidae: Gelechiinae]

rabula Hodges, 1999 (Chionodes); Moths of America north of Mexico, fasc. 7.6: 59. Holotype $\star^{\star}$, USA, Florida, Highlands Co., Parker Island, 26-29 May 1964, R. W. Hodges. [Gelechiidae: Gelechiinae]

radiantella Braun, 1922 (Elachista); Canadian Entomologist 54: 92. Holotype ㅇ, USA, Washington, D.C., 8844, r.f. Panicium, em. July 1899, A. Busck. USNM Genitalia Slide No. 90108. [Elachistidae: Elachistinae]

radiatella Busck, 1904 (Gnorimoschema); Proceedings of the U.S. National Museum 27: 758. Holotype $\sigma^{*}$, USA, Washington, Pullman, August, Piper. USNM Type No. 7857, USNM Genitalia Slide No. 7188, USNM Wing Slide No. 7187. [Gelechiidae: Gelechiinae] 

type 9 , USA, New Jersey, Ocean Co., Lakehurst, 30 May 1962, R.W. Hodges. USNM Genitalia Slide No. 87224. [Coleophoridae: Coleophorinae]

ravicapitis Hodges, 1960 (Martyringa); Bulletin of the Brooklyn Entomological Society 55: 81. Holotype 9 , USA, Louisiana, Alexandria, "feeding on detritus in phasmid fras," 24 February 1959, R. W. Hodges. USNM Genitalia Slide No. 1052. [Oecophoridae: Oecophorinae]

recens Hodges, 1966 (Lita); Proceedings of the U.S. National Museum 119: 31. Holotype *, USA, California, Mt. Shasta, 19 August 1939, E. C. Johnston. [Gelechiidae: Gelechiinae]

rectifex Hodges, 1999 (Chionodes); Moths of America north of Mexico, fasc. 7.6: 199. Holotype $\star^{\star}$, USA, Florida, Escambia Co., 11 January 1963, S. Hills. USNM Genitalia Slide No. 8381. [Gelechiidae: Gelechiinae]

rectistrigella Barnes \& Busck, 1920 (Gelechia); Contributions to the Natural History of the Lepidoptera of North America 4: 229. Holotype 9 , USA, California, Riverside Co. [no date]. USNM Genitalia Slide No. 7484. [Gelechiidae: Gelechiinae]

rector Hodges, 1999 (Chionodes); Moths of America north of Mexico, fasc. 7.6: 83. Holotype $\sigma^{\star}$, USA, Colorado, Chaffee Co., 5 mi N Buena Vista, 8200', 14 July 1982, R. W. Hodges. [Gelechiidae: Gelechiinae]

regens Hodges, 1999 (Chionodes); Moths of America north of Mexico, fasc. 7.6: 85. Holotype $\sigma^{*}$, USA, Nebraska, Cherry Co., Hackberry Lake, Valentine National Wildlife Refuge, 2 June 1983, R. W. Hodges. [Gelechiidae: Gelechiinae]

regiella Busck, 1912 (Peleopoda); Smithsonian Miscellaneous Collections 59: 7. Holotype $\sigma^{*}$, Panama, Cabima, May, A. Busck. USNM Type No. 14534, USNM Genitalia Slide No. 69541. [Peleopodidae]

regina Clarke, 1986 (Asymphorodes); Smithsonian Contributions to Zoology 416: 237. Holotype $o^{*}$, Marquesas Archipelago, Fatu Hiva, Tahuna, 2000', 27 March 1968, J. F. G. \& T. M. Clarke. USNM Type No. 100797, USNM Genitalia Slide No. 24688. [Cosmopterigidae: Cosmopteriginae]

remigiata Clarke, 1986 (Asymphorodes); Smithsonian Contributions to Zoology 416: 235. Holotype $\sigma^{\star}$, Marquesas Archipelago, Nuku Hiva, Taiohae, 17 January 1968, J. F. G. \& T. 
M. Clarke. USNM Type No. 100796, USNM Genitalia Slide No. 24690. [Cosmopterigidae: Cosmopteriginae]

repanda Hodges, 1978 (Periploca); Moths of America north of Mexico, fasc. 6.1: 104. Holotype ${ }^{*}$, USA, Texas, Brewster Co., Big Bend National Park, Chisos Mountains, Nugent Mt., 4 June 1973, R. W. Hodges. USNM Genitalia Slide No. 4724. [Cosmopterigidae: Chrysopeleiinae]

reparabilis Walsingham, 1912 (Glyphidocera); Biologia Centrali-Americana, Lepidoptera Heterocera 4: 112. Holotype $\sigma^{*}$, Panama, Tabernilla, Canal Zone, [no date], A. Busck. USNM Genitalia Slide No. 82505. According to the original description, the holotype of this species is deposited in BMNH. A label accompanying the specimen reads "Glyphidocera vixincisa Wlsm," which apparently is a manuscript name. [Glyphidoceridae]

repartella Dietz, 1910 (Valentinia); Transactions of the American Entomological Society 36: 19. Lectotype ơ, USA, Colorado, Denver, [no date], E. Oslar. USNM Genitalia Slide No. 80987. Right forewing lacking. Designated by Adamksi \& Hodges (1996). [Coleophoridae: Blastobasinae]

repertor Hodges, 1966 (Batrachedra); Transactions of the American Entomological Society 92: 633. Holotype 9 , Panama, Trinidad River, June 1912, A. Busck. USNM Type No. 67868, R. W. Hodges Genitalia Slide No. 3657. [Batrachedridae]

restio Hodges, 1999 (Chionodes); Moths of America north of Mexico, fasc. 7.6: 148. Holotype o*, USA, California, Sonoma Co., Sonoma, 26 June 1937, E. C. Johnston. [Gelechiidae: Gelechiinae]

retiniella Barnes \& Busck, 1920 (Gelechia); Contributions to the Natural History of the Lepidoptera of North America 4: 228. Holotype 9 , USA, Nevada, Verdi [no date]. Abdomen lacking. [Gelechiidae: Gelechiinae]

rhodochra Clarke, 1978 (Nedenia); Smithsonian Contributions to Zoology 273: 40. Holotype ${ }^{*}$, Chile, Llanquihue, Petrohue, 12 March 1959, J. F. G. Clarke. USNM Type no. 73712. [Oecophoridae: Oecophorinae]

rhodograpta Meyrick, 1915 (Filinota); Transactions of the Entomological Society of London 1915: 209. Holotype 9 , British Guiana, Mallali, March 1913, Parish. J. F. G. Clarke Genitalia Slide No. 10969. [Oecophoridae: Oecophorinae] 
rhombus Hodges, 1999 (Chionodes); Moths of America north of Mexico, fasc. 7.6: 105. Holotype ${ }^{*}$, USA, Arizona, Coconino Co., Fort Valley, $7.5 \mathrm{mi}$ NW Flagstaff, 10 July 1961, R. W. Hodges. USNM Genitalia Slide No. 8040. [Gelechiidae: Gelechiinae]

rianella Kaila, 1999 (Elachista); Acta Zoologica Fennica 211: 109. Holotype ơ, USA, Arizona, Coconino Co., Vail Lake Rd., 9.5 mi SE Flagstaff, 6500', 11 July 1961, R. W. Hodges. USNM Genitalia Slide No. 90101. [Elachistidae: Elachistinae]

rigidae Clarke, 1935 (Gelechia); Canadian Entomologist 67: 249. Holotype o*, USA, Washington, Whitman Co., Rock Lake, r.f. Artemisia rigida, em. 2 July 1934, J. F. G. Clarke. USNM Type No. 50945, USNM Genitalia Slide No. 9952. [Gelechiidae: Gelechiinae]

rileyella Chambers, 1872 (Depressaria); Canadian Entomologist 4: 106. Syntype o*, USA, Kentucky [no date]. USNM Type No. 462. Abdomen lacking. A second syntype is in MCZ (Miller \& Hodges 1990). [Gelechiidae: Gelechiinae]

rita Busck, 1920 (Gonioterma); Insecutor Inscitiae Menstruus 8: 92. Holotype o*, British Guiana, [no date], C. M. Beebe. USNM Type No. 22314, USNM Genitalia Slide No. 14145. [Elachistidae: Stenomatinae]

rixator Hodges, 1966 (Batrachedra); Transactions of the American Entomological Society 92: 619. Holotype \%, Colombia (57-2754), 2000 m, Gallego no. 2, r.f. Agave [no date]. USNM Type No. 67863. [Batrachedridae]

roceliella Clarke, 1942 (Filatima); Proceedings of the U.S. National Museum 92: 271. Holotype ${ }^{*}$, USA, Washington, Pullman, 18 July 1930, J. F. G. Clarke. USNM Type No. 56272, J. F. G. Clarke Genitalia Slide No. 2926. [Gelechiidae: Gelechiinae]

rogator Hodges, 1999 (Chionodes); Moths of America north of Mexico, fasc. 7.6: 208. Holotype $\sigma^{*}$, USA, Colorado, Alamosa Co., Great Sand Dunes National Monument, Mosca Creek, 8200', 28 June 1982, R. W. Hodges. [Gelechiidae: Gelechiinae]

rosa Busck, 1911 (Gonioterma); Proceedings of the U.S. National Museum 40: 224. Holotype ${ }^{\star}$, French Guiana, St. Jean, Maroni River, [no date], W. Schaus. USNM Type No. 13590, USNM Genitalia Slide No. 13917. [Elachistidae: Stenomatinae]

rosaciliella Busck, 1904 (Depressaria); Proceedings of the U.S. National Museum 27: 763. Syntype 9 , USA, Oregon, Camp Watsia, April 1872, Walsingham. USNM Type No. 7815. Abdomen lacking. Other syntypes not found. [Oecophoridae: Depressariinae] 
rosapicella Busck, 1914 (Rhindoma); Proceedings of the U.S. National Museum 47: 25. Holotype $o^{*}$, Panama, Alhajuelo, April 1911, A. Busck. USNM Type No. 16684, USNM

Genitalia Slide No. 69450. [Oecophoridae: Oecophorinae]

roseicorpus Dognin, 1910 (Oecophora); Heteroceres Nouveaux l'Amerique du Sud 24: 45. Holotype $\sigma^{*}$, French Guiana, St. Laurent on Maroni, “12.6.12," Durrant, Dognin Collection. USNM Type No. 32349. [Oecophoridae: Oecophorinae]

roseicrinella Busck, 1909 (Sophronia); Proceedings of the Entomological Society of Washington 11: 87. Holotype $\star^{\star}$, USA, Texas, Kerrville, [no date], F. C. Pratt. USNM Type no. 12262, USNM Genitalia Slide No. 86517. [Gelechiidae: Gelechiinae]

roseomarginella Busck, 1911 (Cryptolechia); Proceedings of the U.S. National Museum 40: 212. Holotype ${ }^{\circ}$, French Guiana, St. Jean, Maroni River [no date]. USNM Type No. 13566, USNM Genitalia Slide No. 69474. [Elachistidae: Depressariinae]

ruba Duckworth, 1964 (Lethata); Proceedings of the U.S. National Museum 116: 108. Holotype $o^{\star}$, Brazil, Nova Teutônia, 3500 m, 21 ${ }^{\circ} 11^{\prime} 8$ "S, 52²3'W, 6 August 1948, F. Plaumann. USNM Genitalia Slide No. 13399. [Elachistidae: Stenomatinae]

rudbeckiella Bottimer, 1926 (Isophrictis); Journal of Agricultural Research 33: 810. Holotype ${ }^{\star}$, USA, Texas, Liberty, r.f. Rudbeckia maxima, June 1924. USNM Type No. 27334, USNM Genitalia Slide No. 5619. [Gelechiidae: Gelechiinae]

rufusella Chambers, 1874 (Gelechia); Canadian Entomologist 6: 240. Holotype ${ }^{*}$, USA, Texas [no date]. USNM Type No. 463, USNM Genitalia Slide No. 10675. [Gelechiidae: Gelechiinae]

rustica Clarke, 1978 (Retha); Smithsonian Contributions to Zoology 273: 58. Holotype ox, Chile, Llanquihue, Petrohue, 10 March 1959, J. F. G. Clarke. USNM Type No. 73723, USNM Genitalia Slide No. 24287. [Oecophoridae: Oecophorinae]

saccharella Busck, 1934 (Donacivola); Entomologica Americana 13: 169. Holotype Cuba, No. 9633, blotchminer on sugar cane. USNM Genitalia Slide No. 90109. [Elachistidae: Elachistinae]

sagax Busck, 1914 (Stenoma); Proceedings of the U.S. National Museum 47: 40. Holotype , Panama, Porto Bello, May 1912, A. Busck. USNM Type No. 16712, W. D. Duckworth Genitalia Slide No. 2062. [Elachistidae: Stenomatinae] 

Co., Taihoku, 21 May 1946, S. Issiki. USNM Genitalia Slide No. 87384. [Gelechiidae: Dichomeridinae]

salinae Walsingham, 1911 (Glyphidocera); Biologia Centrali-Americana, Lepidoptera Heterocera 4: 110. Holotype \%, Mexico, Salina Cruz, [no date], W. Schaus. Abdomen lacking. [Glyphidoceridae]

salinaris Busck, 1911 (Gnorimoschema); Canadian Entomologist 43: 4. Holotype $q$, USA, Massachusetts, Boston, r.f. Solidago sempervirens, em. 4 September 1909. USNM Type No. 13441, USNM Genitalia Slide No. 7037. [Gelechiidae: Gelechiinae]

salome Busck, 1911 (Stenoma); Proceedings of the U.S. National Museum 40: 219. Holotype $q$, Brazil, Paraná, Castro, [no date], W. Schaus. USNM Type No. 13580, Genitalia Slide AB Jan. 10, 1920. [Elachistidae: Stenomatinae]

sanator Hodges, 1999 (Chionodes); Moths of America north of Mexico, fasc. 7.6: 85. Holotype $\sigma^{*}$, USA, Arizona, Cochise Co., Chiricahua Mountains, Southwest Research Station, 5400', 5-8 July 1964, D. R. Davis. USNM Genitalia Slide No. 7754. [Gelechiidae: Gelechiinae]

sandaradema Wu, 1998 (Tisis); Zoological Studies 37: 192. Holotype ơ, Sarawak, Semengob Forest Reserve, $15 \mathrm{mi} \mathrm{S}$ of Kuching, 16 September 1966, J. F. G. \& T. M. Clarke. USNM Genitalia Slide No. 89257. [Lecithoceridae]

sandra Powell, 1973 (Ethmia); Smithsonian Contributions to Zoology 120: 166. Holotype ơ, El Salvador, 13 km N San Salvador, 4 February 1965, S. S. \& W. D. Duckworth. [Elachistidae: Ethmiinae]

sanguinella Busck, 1902 (Depressaria); Proceedings of the U.S. National Museum 24: 738. Holotype $\diamond^{\star}$, USA, Arizona, Pinal Mts., July 1900, R. Kunzé. USNM Type No. 6129, USNM Genitalia Slide No. 260. [Elachistidae: Depressariinae]

sannio Hodges, 1999 (Chionodes); Moths of America north of Mexico, fasc. 7.6: 70. Holotype \&, USA, Washington, Yakima Co., $2.5 \mathrm{mi}$ W Fort Simcoe, 31 July 1962, J. F. G. Clarke. USNM Genitalia Slide No. 14759. [Gelechiidae: Gelechiinae]

santarosensis Hodges, 1985 (Dichomeris); Proceedings of the Entomological Society of Washington 87: 456. Holotype $\sigma^{*}$, Costa Rica, Guanacaste Province, Santa Rosa National Park, r.f. Quercus oleoides, 82.SRNP.311, D. H. Janzen. USNM Genitalia Slide No. 12099. [Gelechiidae: Dichomeridinae] 
sarissa Clarke, 1978 (Alynda); Smithsonian Contributions to Zoology 273: 55. Holotype

o* Chile, Centro-Austral, January-March 1898, V. Izquerdo. USNM Type No. 73720,

USNM Genitalia Slide No. 24284. Right fore- and hindwing lacking. [Oecophoridae: Oecophorinae]

sattleri Hodges, 1999 (Chionodes); Moths of America north of Mexico, fasc. 7.6: 218. Holotype $\sigma^{x}$, Canada, Nova Scotia, Halifax Co., Halifax Watershed, Bog E Big Indian Lake, 21 July 1968, D. C. Ferguson. [Gelechiidae: Gelechiinae]

scabrella Busck, 1913 (Galechia) [sic]; Journal of Entomology and Zoology (Pomona College) 5: 96. Syntype (uncertain sex), USA, California, San Diego, June-July, W. S. Wright. Specimen not found. [Gelechiidae: Gelechiinae]

schellbachi Clarke, 1947 (Depressaria); Journal of the Washington Academy of Sciences 37: 10. Holotype $\sigma^{*}$, USA, Arizona, Grand Canyon, Shoshone Point, 7050', 18 June 1944, L. Schellbach. USNM Type No. 58013, USNM Genitalia Slide No. 580. [Elachistidae: Depressariinae]

schwarziella Busck, 1901 (Euclemensia); Journal of the New York Entomological Society 8 (1900): 239. Lectotype + , USA, Arizona, Santa Rita Mountains, r.f. Kermes sp. on oak, em. 20 June 1898. USNM Type No. 5356, USNM Genitalia Slide No. 7781. Designated by Hodges (1978). [Cosmopterigidae: Antequerinae]

scirpicola Hodges, 1962 (Cosmopterix); Entomologica Americana (n.s.) 42: 49. Holotype ๙, USA, Oklahoma, Oklahoma City, 13 August 1956, D. R. Davis. USNM Type No. 66080, USNM Genitalia Slide No. 3913. [Cosmopterigidae: Cosmopteriginae]

scitator Hodges, 1966 (Batrachedra); Transactions of the American Entomological Society 92: 612. Holotype o*, USA, Arizona, Gila Co., 4 mi ESE Pine, 5400', 5 September 1961, R. W. Hodges. USNM Type No. 67860, USNM Genitalia Slide No. 5096. [Batrachedridae]

scobifera Kaila, 1997 (Elachista); Acta Zoologica Fennica 206: 42. Holotype o*, USA, Arizona, Coconino Co., 7.5 mi NW Flagstaff, Fort Valley, 7350', 28 August 1961, R. W. Hodges. USNM Genitalia Slide No. 89279. [Elachistidae: Elachistinae]

scrutaria Hodges, 1986 (Dichomeris); Moths of America north of Mexico, fasc. 7.1: 93. Holotype $\varnothing^{\star}$, USA, Louisiana, St. Tam. Parish, $4.2 \mathrm{mi}$ NE Abita Springs, T6S R12E S24, 15 September 1983, V. Brou. [Gelechiidae: Dichomeridinae] 

cera 4: 148. Holotype $\sigma^{\star}$, Costa Rica, Banana River, March 1907, W. Schaus. USNM Type No. 68206. [Elachistidae: Ethmiinae]

seculaella Clarke, 1932 (Gelechia); Canadian Entomologist 64: 67. Holotype $\sigma^{\star}$, USA, Washington, Pullman, 24 April 1926, J. F. G. Clarke. USNM Type No. 15610, USNM Genitalia Slide No. 10154. [Gelechiidae: Gelechiinae]

secura Hodges, 1964 (Nepotula); Proceedings of the U.S. National Museum 115: 296. Holotype $\sigma^{x}$, USA, Florida, Pensacola, 28 October 1961, S. Hills. USNM Genitalia Slide No. 4472, USNM Wing Slide No. 4473. [Cosmopterigidae: Chrysopeleiinae]

secutor Hodges, 1999 (Chionodes); Moths of America north of Mexico, fasc. 7.6: 146. Holotype $\sigma^{\star}$, USA, Texas, Jeff Davis Co., Davis Mountains, 5 mi SE Livermore, 29 August 1970, A. \& M. E. Blanchard. [Gelechiidae: Gelechiinae]

sedulitella Busck, 1910 (Gelechia); Proceedings of the Entomological Society of Washington 11: 181. Holotype $\sigma^{*}$, USA, California, Placer Co., August-September, A. Koebele. USNM Type No. 12696, USNM Genitalia Slide No. 6674. [Gelechiidae: Gelechiinae]

selene Clarke, 1971 (Gonionota); Smithsonian Contributions to Zoology 95: 27. Holotype ${ }^{\circ}$, Brazil, Santa Catarina, 21 January 1936, F. Plaumann. USNM Type No. 71099, USNM Genitalia Slide No. 68909. [Oecophoridae: Oecophorinae]

semibrunnea Dognin, 1905 (Cryptolechia); Annales de la Société Entomologique de Belgique 49: 88. Holotype $\sigma^{*}$, Ecuador, environs de Loja, Dognin Collection. USNM Type No. 32359, USNM Genitalia Slide No. 4084. [Elachistidae: Depressariinae]

semicyclionella Busck, 1903 (Gnorimoschema); Proceedings of the U.S. National Museum 25: 828. Holotype ơ, USA, New Mexico, Las Vegas HS, 5 August, H. S. Barber. USNM Type No. 6360. [Gelechiidae: Gelechiinae]

semiluteus Clarke, 1986 (Asymphorodes); Smithsonian Contributions to Zoology 416: 213. Holotype $\sigma^{\star}$, Marquesas Archipelago, Hiva Oa, Mt. Feani, 3800', 20 February 1968, J. F. G. \& T. M. Clarke. USNM Type No. 100791, USNM Genitalia Slide No. 24844. [Cosmopterigidae: Cosmopteriginae]

seminiger Clarke, 1986 (Asymphorodes); Smithsonian Contributions to Zoology 416: 211. Holotype $\sigma^{*}$, Marquesas Archipelago, Nuku Hiva, Tapuaooa, 2500', 30 January 1968, J. F. G. \& T. M. Clarke. USNM Type No. 100786. [Cosmopterigidae: Cosmopteriginae] 
semiombra Dyar, 1902 (Ethmia); Journal of the New York Entomological Society 10: 206. Syntype + , USA, California, San Diego, 12 June 1895, E. A. Schwarz. USNM Type No. 6623. [Elachistidae: Ethmiinae]

semitenebrella Dyar, 1902 (Ethmia); Journal of the New York Entomological Society 10: 204. Lectotype + , USA, Arizona, Fort Grant, [no date], H. G. Hubbard. USNM Genitalia Slide No. 98448. Designated by Powell (1973). [Elachistidae: Ethmiinae]

senica Hodges, 1999 (Chionodes); Moths of America north of Mexico, fasc. 7.6: 206. Holotype ${ }^{\star}$, USA, Arizona, Coconino Co., Hart Prairie, 10 mi NNW Flagstaff, 18 August 1961, R. W. Hodges. USNM Genitalia Slide No. 7976. [Gelechiidae: Gelechiinae]

senicionella Busck, 1902 (Depressaria); Proceedings of the U.S. National Museum 24: 742. Lectotype $\sigma^{*}$, USA, Colorado, r.f. Umbelliferae, H. Dyar. USNM Type No. 6126. Abdomen lacking; head in gelatin capsule. Designated by Hodges (1974). Abdomen lacking. [Elachistidae: Depressariinae]

septentrionella Busck, 1904 (Glyphidocera); Proceedings of the U.S. National Museum 27: 762. Holotype $\diamond^{\star}$, Canada, British Columbia, Kaslo [no date]. USNM Type No. 7865, USNM Genitalia Slide No. 10636. [Glyphidoceridae]

sepultor Hodges, 1999 (Chionodes); Moths of America north of Mexico, fasc. 7.6: 220. Holotype $\sigma^{*}$, USA, Wyoming, Weston Co., 6 mi NW Newcastle, 23 June 1965, R. Hodges. [Gelechiidae: Gelechiinae]

sequella Busck, 1914 (Triclonella); Proceedings of the U.S. National Museum 47: 32. Holotype 9 , Panama, Porto Bello, April, A. Busck. USNM Type No. 16692. [Cosmopterigidae: Cosmopteriginae]

serica Kaila, 1995 (Mendesia); Journal of the Lepidopterists' Society 49: 219. Holotype ${ }^{*}$, USA, Colorado, Chaffee Co., 1 mi S Poncha Springs, 7000', pinyon-juniper/oak-cottonwood/willow-Rhus, 6 July 1982, R. W. Hodges. USNM Genitalia Slide No. 89301. [Elachistidae: Elachistinae]

sericeus Clarke, 1986 (Asymphorodes); Smithsonian Contributions to Zoology 416: 261. Holotype $\sigma^{\star}$, Marquesas Archipelago, Nuku Hiva, Tunoa Ridge, 2900', 23 January 1968, J. F. G. \& T. M. Clarke. USNM Type No. 100799. [Cosmopterigidae: Cosmopteriginae]

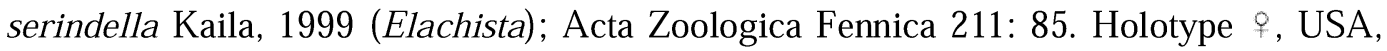
Arizona, Madera Canyon, Santa Rita Mts., 4880', 16 August 1959, R. W. Hodges. USNM Genitalia Slide No. 90107. [Elachistidae: Elachistinae] 
serotinella Busck, 1903 (Gelechia); Proceedings of the U.S. National Museum 25: 881. Holotype $\sigma^{\star}$, USA, District of Columbia, r.f. Prunus serotina [no date]. USNM Type No. 6390, USNM Genitalia Slide No. 9668. [Gelechiidae: Gelechiinae]

serrae Clarke, 1933 (Agonopterix); Canadian Entomologist 65: 84. Holotype o*, USA, Washington, Pullman, 24 June 1932, r.f. Senecio serra, J. F. G. Clarke. USNM Type No. 44740, USNM Genitalia Slide No. 182. [Elachistidae: Depressariinae]

serrata Wu \& Park, 1999 (Frisilia); Korean Journal of Systematic Zoology 15: 2. Holotype $\sigma^{\star}$, Sri Lanka, Anu. Dist., Hunuwilagama, near Wilpattu, 200', 28 October-3 November 1976, black light, Hevel, Dietz, Karunaratne \& Balasooriya. [Lecithoceridae]

servilis Walsingham, 1911 (Dichomeris); Biologia Centrali-Americana, Lepidoptera Heterocera 4: 103. Holotype + , Panama, Canal Zone, Tabernilla, June 1907, A. Busck, Walsingham No. 5786. [Gelechiidae: Dichomeridinae]

sevir Hodges, 1999 (Chionodes); Moths of America north of Mexico, fasc. 7.6: 137. Holotype $\diamond^{\star}$, USA, North Carolina, Macon Co., Highlands, 3865', 5 July 1958, J. G. Franclemont. [Gelechiidae: Gelechiinae]

sexmaculata Dognin, 1904 (Cryptolechia); Annales de la Société Entomologique de Belgique 48: 133. Holotype ․, Ecuador, San Francisco, 2000 m, “12.6.12,” Durrant, Dognin Collection. USNM Type No. 32365, USNM Genitalia Slide No. 14036. [Elachistidae: Stenomatinae]

similatella Busck, 1920 (Ethmia); Insecutor Inscitiae Menstruus 8: 83. Holotype o*, Guatemala, Cayuga, [no date], W. Schaus. USNM Type No. 22304. [Elachistidae: Ethmiinae]

similis Busck, 1911 (Stenoma); Proceedings of the U.S. National Museum 40: 222. Holotype $\sigma^{*}$, French Guiana, St. Jean, Maroni River, [no date], W. Schaus. USNM Type No. 13588, USNM Genitalia Slide No. 17833. [Elachistidae: Stenomatinae]

simplex Busck, 1914 (Stenoma); Proceedings of the U.S. National Museum 47: 49. Holotype $\sigma^{*}$, Panama, Trinidad River, June 1912, A. Busck. USNM Type No. 16738. Abdomen lacking. [Elachistidae: Stenomatinae]

simpliciella Busck, 1904 (Trichotaphe); Proceedings of the U.S. National Museum 27: 761. Holotype $\sigma^{\star}$, USA, Washington, Pullman, 10 August 1898, C. V. Piper. USNM Type No. 7863, USNM Genitalia Slide No. 10645. [Gelechiidae: Dichomeridinae] 
simulata Hodges, 1986 (Dichomeris); Moths of America north of Mexico, fasc. 7.1: 104. Holotype $\diamond^{\star}$, USA, Texas, Hemphill Co., Canadian, 28 May 1970, A. \& M. E. Blanchard. USNM Genitalia Slide No. 12309. Left forewing in gelatin capsule. [Gelechiidae: Dichomeridinae]

simulatrix Hodges, 1978 (Ithome); Moths of America north of Mexico, fasc. 6.1: 137. Holotype ${ }^{*}$, USA, Florida, Everglades, 8-15 April. USNM Genitalia Slide No. 1372. [Cosmopterigidae: Chrysopeleiinae]

simulella Dietz, 1910 (Holcocera); Transactions of the American Entomological Society 36: 52. Lectotype + , USA, Arizona, Williams, 17 July, H. S. Barber. USNM Genitalia Slide No. 11102. Designated by Adamski \& Hodges (1996). [Coleophoridae: Blastobasinae]

sinelinea Hodges, 1978 (Cosmopterix); Moths of America north of Mexico, fasc. 6.1: 24. Holotype ơ, USA, South Carolina, McClellanville, The Wedge, 26 August 1971, R. W. Hodges. USNM Genitalia Slide No. 3772. [Cosmopterigidae: Cosmopteriginae]

siren Hodges, 1986 (Dichomeris); Moths of America north of Mexico, fasc. 7.1: 64. Holotype $\diamond^{\star}$, USA, Maryland, Prince Georges Co., Oxon Hill, Henson Creek, 7 August 1978, D. R. Davis. [Gelechiidae: Dichomeridinae]

sironae Hodges, 1966 (Lita); Proceedings of the U.S. National Museum 119: 27. Holotype $\checkmark$, USA, San Diego Co., San Diego [no date]. USNM Type No. 67649, USNM Genitalia Slide No. 7499. [Gelechiidae: Gelechiinae]

sistrella Busck, 1903 (Gelechia); Proceedings of the U.S. National Museum 25: 862. Lectotype ${ }^{*}$, USA, Arizona, Phoenix, August, Kunze. USNM Type No. 6377, USNM Genitalia Slide No. 8048. Designated by Hodges (1999). [Gelechiidae: Gelechiinae]

slabaughi Miller, 2000 (Gnorimoschema); Thomas Say Publications in Entomology: 42. Holotype + , USA, North Dakota, Mercer Co., T147N R85W S6, r.f. Grindelia squarrosa, October 1970, P. E. Slabaugh. USNM Genitalia Slide No. 90898. [Gelechiidae: Gelechiinae]

smogops Hodges, 1978 (Anoncia); Moths of America north of Mexico, fasc. 6.1: 72. Holotype 9 , Arizona, Pima Co., Baboquivari Mountains, 1-15 May 1924, O. C. Poling. USNM Genitalia Slide No. 4181. [Cosmopterigidae: Cosmopteriginae] 
solanophaga Adamski \& Brown, 2002 (Frumenta); Proceedings of the Entomological Society of Washington 104: 1030. Holotype ${ }^{*}$, Mexico, San Luis Potosí, San Luis de la Paz, 21.19N, 100.32W, 10 September 1999, r.f. Solanum elaeagnifolium, H. G. Zimmerman. [Gelechiidae: Gelechiinae]

solomoni Wagner, Adamski \& Brown, 2004 (Mompha); Proceedings of the Entomological Society of Washington 106: 2. Holotype ${ }^{\star}$, USA, Mississippi, Washington Co., Stoneville, r.f. Cephalanthus occidentalis (twig borer), May 1987, J. D. Solomon. [Coleophoridae: Momphidae]

soronella Busck, 1914 (Arogalea); Proceedings of the U.S. National Museum 47: 17. Holotype + , Panama, La Chorrera, May, A. Busck. USNM Type No. 15833, USNM Genitalia Slide No. 10666. [Gelechiidae: Gelechiinae]

soror Hodges, 1978 (Periploca); Moths of America north of Mexico, fasc. 6.1: 108. Holotype + , Texas, Brewster Co., Big Bend National Park, Chisos Mountains, Green Gulch, 5500', 6 June 1973, R. W. Hodges. USNM Genitalia Slide No. 4767. [Cosmopterigidae: Chrysopeleiinae]

soter Hodges, 1999 (Chionodes); Moths of America north of Mexico, fasc. 7.6: 129. Holotype $\sigma^{\star}$, USA, Illinois, Putnam Co., 10 July 1962, M. O. Glenn. [Gelechiidae: Gelechiinae]

sparsa Hodges, 1978 (Melanocinclis); Moths of America north of Mexico, fasc. 6.1: 42. Holotype $\sigma^{\star}$, USA, Arkansas, Washington Co., Devil's Den State Park, 6 August 1966, R. W. Hodges. USNM Type No. 70567, USNM Genitalia Slide No. 5584. [Cosmopterigidae: Cosmopteriginae]

speratella Busck, 1908 (Glyphidocera); Proceedings of the Entomological Society of Washington 9: 88. Holotype ${ }^{*}$, USA, Pennsylvania, New Brighton, 4 July 1907, Merrick. USNM Type No. 11324, USNM Genitalia Slide No. 10640. [Glyphidoceridae]

speratum Busck, 1911 (Stenoma); Proceedings of the U.S. National Museum 40: 219. Holotype ${ }^{\star}$, French Guiana, Cayenne, [no date], W. Schaus. USNM Type No. 13579, USNM Genitalia Slide No. 13657. [Elachistidae: Stenomatinae]

sperryi Clarke, 1947 (Filatima); Journal of the Washington Academy of Sciences 37: 270. Holotype ${ }^{*}$, USA, California, San Bernardino Co., San Bernardino Mountains, Barton Flats, 4 August 1945, A. L. Melander. USNM Type No. 58251, USNM Genitalia Slide No. 9672. [Gelechiidae: Gelechiinae] 
sphaeralceae Clarke, 1941 (Martyrhilda); Proceedings of the U.S. National Museum 90: 138. Holotype ${ }^{\star}$, USA, Washington, Grant Co., Grand Coulee, Park Lake, 15 May 1935, r.f. Sphaeralcea munroana, J. F. G. Clarke. USNM Type No. 52076, USNM Genitalia Slide No. 938. [Elachistidae: Depressariinae]

sphenogramma Clarke, 1971 (Gonionota); Smithsonian Contributions to Zoology 95: 25. Holotype ${ }^{\star}$, Venezuela, Aragua, Rancho Grande, 1100 m, 16-19 January 1966, S. S. \& W. D. Duckworth. USNM Type No. 71098, USNM Genitalia Slide No. 68905. [Oecophoridae: Oecophorinae]

spilosella Barnes \& Busck, 1920 (Gelechia); Contributions to the Natural History of the Lepidoptera of North America 4: 228. Holotype (uncertain sex), USA, California, San Diego Co., San Diego [no date]. Specimen not found. [Gelechiidae: Gelechiinae]

spinigera Clarke, 1947 (Filatima); Journal of the Washington Academy of Sciences 37: 274. Holotype ${ }^{*}$, USA, California, Mojave Desert, near Llano, 4 April 1938, J. A. Comstock. USNM Type No. 58247, USNM Genitalia Slide No. 9678. [Gelechiidae: Gelechiinae]

splendoriferella Busck, 1904 (Gnorimoschema); Proceedings of the U.S. National Museum 27: 756. Holotype ${ }^{*}$, USA, Washington, Pullman, August, Piper. USNM Type No. 7858, USNM Genitalia Slide No. 7029. [Gelechiidae: Gelechiinae]

spodogramma Clarke, 1986 (Asymphorodes); Smithsonian Contributions to Zoology 416: 266. Holotype ${ }^{\star}$, Marquesas Archipelago, Hiva Oa, Atuona, 10 February 1968, J. F. G. \& T. M. Clarke. USNM Type No. 100821, USNM Genitalia Slide No. 25141. [Cosmopterigidae: Cosmopteriginae]

sponsella Busck, 1907 (Colinita); Journal of the New York Entomological Society 15: 139. Holotype ${ }^{\star}$, USA, Arizona, Yuma Co., desert. USNM Type No. 10324, USNM Genitalia Slide No. 86628, USNM Wing Slide No. 86629. [Xyloryctidae: Scythridinae]

sponsus Hodges, 1999 (Chionodes); Moths of America north of Mexico, fasc. 7.6: 207. Holotype $\sigma^{\star}$, USA, Texas, Culberson Co., Sierra Diablo Wildlife Management Area, 6400', 30 August 1970, J. G. Franclemont. USNM Genitalia Slide No. 11885 [Gelechiidae: Gelechiinae]

stator Hodges, 1999 (Chionodes); Moths of America north of Mexico, fasc. 7.6: 71. Holotype \& USA, Washington, Yakima Co., 2.5 mi W Fort Simcoe, 31 July 1962, J. F. G. Clarke. USNM Genitalia Slide No. 14663. [Gelechiidae: Gelechiinae] 

type $\sigma^{*}$, USA, Massachusetts, Martha's Vineyard, 17 July 1949, F. M. Jones. USNM Genitalia Slide No. 1160. Left forewing lacking. [Oecophoridae: Oecophorinae]

stella Busck, 1911 (Gonioterma); Proceedings of the U.S. National Museum 40: 225. Holotype $\sigma^{*}$, French Guiana, St. Jean, Maroni River, [no date], W. Schaus. USNM Type No. 13592, USNM Genitalia Slide No. 4017. [Elachistidae: Stenomatinae]

stellatella Busck, 1914 (Dichomeris); Proceedings of the U.S. National Museum 47: 20. Holotype $\sigma^{\star}$, Panama, Taboga Island, February 1912, A. Busck. USNM Type No. 16678. Right fore- and hindwing lacking. [Gelechiidae: Dichomeridinae]

stellella Busck, 1906 (Mompha); Canadian Entomologist 38: 123. Holotype ${ }^{\star}$, USA, Washington, D.C., em. 5 August 1892, USNM Type No. 9796. According to the original description, larvae feed at the base of the flowers of Oenothera sp. [Coleophoridae: Momphinae]

stenomacra Wu \& Park, 1999 (Timyra); Korean Journal of Systematic Zoology 15: 211. Holotype ${ }^{*}$, Sri Lanka, Kan. District, Udawattakele, 1800', 19 November 1976, black light, Hevel, Dietz, Karunaratne \& Balasooriya. USNM Genitalia Slide No. 90122. Specimen not found. [Lecithoceridae]

stipator Hodges, 1964 (Aeaea); Proceedings of the U.S. National Museum 115: 303. Holotype $\sigma^{\star}$, USA, Arizona, Coconino Co., Vail Lake Road, $9.5 \mathrm{mi}$ SE Flagstaff, 6500', 11 July 1961, R. W. Hodges. USNM Genitalia Slide No. 9595. [Cosmopterigidae: Chrysopeleiinae]

stomatocosma Meyrick, 1932 (Stenoma); Exotic Microlepidoptera 4: 304. Holotype ox, Brazil, Teffé, Amazones, September, Fassel. Genitalia Slide AB Dec. 1, 1937. [Elachistidae: Stenomatinae]

stonda Hodges, 1974 (Decantha); Moths of America north of Mexico, fasc. 6.2: 108. Holotype $o^{\star}$, USA, Wyoming, 6 mi NW Newcastle, 13 July 1965, R. W. Hodges. [Oecophoridae: Oecophorinae]

stratia Hodges, 1974 (Himmacia); Moths of America north of Mexico, fasc. 6.2: 83. Holotype $\sigma^{*}$, USA, Arizona, Santa Cruz Co., Santa Rita Mountains, Madera Canyon, 5600', 14 June 1965, J. G. Franclemont. [Oecophoridae: Oecophorinae] 
striata Clarke, 1978 (Alynda); Smithsonian Contributions to Zoology 273: 54. Holotype $o^{x}$, Chile, Arauco, Caramavida, 1-6 January 1954, L. E. Peña. USNM Type No. 73719. [Oecophoridae: Oecophorinae]

striatella Busck, 1903 (Gelechia); Proceedings of the U.S. National Museum 25: 869. Holotype ${ }^{*}$, USA, Arizona [no date]. USNM Type No. 6382, USNM Genitalia Slide No. 9689. [Gelechiidae: Gelechiinae]

striatella Busck, 1913 (Ethmia); Insecutor Inscitiae Menstruus 1: 141. Holotype ${ }^{*}$, Mexico, Tehuacan, June, R. Müller. USNM Type No. 16662. [Elachistidae: Ethmiinae]

striatella Busck, 1914 (Stenoma); Proceedings of the U.S. National Museum 47: 47. Holotype ?, Panama, Alhajuela, April 1911, A. Busck. USNM Type No. 16726, USNM Genitalia Slide No. 13966. [Elachistidae: Stenomatinae]

suasor Hodges, 1999 (Chionodes); Moths of America north of Mexico, fasc. 7.6: 57. Holotype $\sigma^{*}$, USA, Texas, Walker Co., Huntsville State Park, 20 May 1969, A. \& M. E. Blanchard. USNM Genitalia Slide No. 82490. [Gelechiidae: Gelechiinae]

suavella Kaila, 1999 (Elachista); Acta Zoologica Fennica 211: 89. Holotype ㅇ, USA, Arizona, Madera Canyon, Santa Rita Mts., 4880', 31 August 1959, R. W. Hodges. USNM Genitalia Slide No. 90103. [Elachistidae: Elachistinae]

subicula Clarke, 1978 (Doina); Smithsonian Contributions to Zoology 273: 28. Holotype ๙, Chile, Llanquihue, Peulla, 7 March 1959, J. F. G. Clarke. USNM Type No. 73705. [Amphisbatidae]

subiridescens Walsingham, 1882 (Laverna); Transactions of the American Entomological Society 1882: 194. Holotype $\sigma^{\star}$, USA, [no other data], Fernald Collection. Abdomen lacking. [Coleophoridae: Momphinae]

submissa Busck, 1914 (Ethmia); Insecutor Inscitiae Menstruus 2: 57. Holotype 우 Cuba, [no date], E. A. Schwarz \& W. Schaus. USNM Type No. 18271, USNM Genitalia Slide No. 87688. [Elachistidae: Ethmiinae]

subnigritaenia Powell, 1973 (Ethmia); Smithsonian Contributions to Zoology 120: 173. Holotype $\sigma^{\star}$, Mexico, Mexico City, [no date], R. Müller. USNM Genitalia Slide No. 87683. [Elachistidae: Ethmiinae] 

Ponte Nova, Rio Xingu, Amazones, [no date], Dognin. Genitalia Slide AB Nov. 3, 1926. Anterior part of abdomen in gelatin capsule. [Elachistidae: Stenomatinae]

subterraneum Busck, 1911 (Gnorimoschema); Canadian Entomologist 43: 5. Syntypes (19), USA, Massachusetts, Boston, r.f. Aster multiflorus, [no date], C. H. Clarke. USNM Type No. 13386. [Gelechiidae: Gelechiinae]

subtusvena Diakonoff, 1968 (Amorbaea); Bulletin of the U.S. National Museum 257 (1967): 115. Holotype ${ }^{*}$, Philippine Islands, Luzon, Mt. Makiling, [no date], P. J. Baker. USNM Genitalia Slide No. 89985. [Xyloryctidae: Xyloryctinae]

subusta Diakonoff, 1968 (Amorbaea); Bulletin of the U.S. National Museum 257 (1967): 116. Holotype ${ }^{*}$, Philippine Islands, Luzon, Mt. Makiling, [no date], P. J. Baker. USNM Genitalia Slide No. 89986. [Xyloryctidae: Xyloryctinae]

sulphurea Busck, 1914 (Cryptolechia); Proceedings of the U.S. National Museum 47: 28. Holotype + , Panama, Porto Bello, June 1911, A. Busck. USNM Type No. 16748, USNM Genitalia Slide No. 69469. [Elachistidae: Depressariinae]

surrepta Diakonoff, 1968 (Homaloxestis); Bulletin of the U.S. National Museum 257 (1967): 122. Holotype $\sigma^{\star}$, Philippine Islands, Luzon, Mt. Apo 70 B, 8 May 1945, J. G. Franclemont. USNM Genitalia Slide No. 89996. [Lecithoceridae]

swezeyi Busck, 1914 (Petrochroa); Insecutor Inscitiae Menstruus 2: 105. Syntypes ( 3 , 1 of uncertain sex), Hawaii, Kaimuki, Oahu, r.f. case on rocks, O. Swezey. USNM Type No. 18430. All syntypes mounted on a single cork block. [Elachistidae: Elachistinae]

swezeyi Clarke, 1984 (Elaeonoma); Insects of Micronesia 9: 151. Holotype + , Southern Mariana Islands, Guam, Piti, 26 September 1936, r.f. dead Barleria, O. H. Swezey. USNM Genitalia Slide No. 24518. [Oecophoridae: Oecophorinae]

sylphe Hodges, 1986 (Dichomeris); Moths of America north of Mexico, fasc. 7.1: 58. Holotype ${ }^{*}$, USA, Florida, Highlands Co., Archbold Biological Station, 1 April 1959, R. W. Hodges. [Gelechiidae: Dichomeridinae]

sylvicolella Busck, 1903 (Epithectis); Proceedings of the U.S. National Museum 25: 818. Holotype $\sigma^{*}$, USA, New York, [no date], Fitch. USNM Type No. 6358, USNM Genitalia Slide No. 6595. [Gelechiidae: Gelechiinae] 
symmetrica Park \& Hodges, 1995 (Dichomeris); Insecta Koreana 20: 16. Holotype ${ }^{*}$, Taiwan, Taitung Co., Orchid Island, 4 km SW Hungta, 16-20 May 1980, D. Davis. USNM

Genitalia Slide No. 87351. [Gelechiidae: Dichomeridinae]

symmorpha Braun, 1948 (Elachista); Memoirs of the American Entomological Society 13: 36. Holotype $\sigma^{*}$, USA, California, Tulare Co., Monache Meadows, 8000', 1-7 July. USNM Type No. 34826, USNM Genitalia Slide No. 89274. [Elachistidae: Elachistinae]

sympatrica Clarke, 1962 (Homoeoprepes); Proceedings of the U.S. National Museum 113: 381. Holotype ${ }^{\star}$, Colombia, Cauca, Paramo de Parace, Lake San Rafael, 3570 m, 27 January 1959, J. F. G. Clarke. USNM Genitalia Slide No. 69030. [Elachistidae: Agonoxeniinae]

syntoma Walsingham, 1912 (Hyphypena); Biologia Centrali-Americana, Lepidoptera Heterocera 4: 131. Holotype ${ }^{\star}$, Panama, Canal Zone, Tabernilla, June 1908, A. Busck. Abdomen lacking. In the original description the date is reported as June 1907. [Oecophoridae: Oecophorinae]

taboga Busck, 1914 (Epicallima); Proceedings of the U.S. National Museum 47: 33. Holotype $\diamond^{\star}$, Panama, Taboga Island, June, A. Busck. USNM Type No. 16694, USNM Genitalia Slide No. 4092. [Oecophoridae: Oecophorinae]

taipingensis Park, 2003 (Issikiopteryx); Journal of Asia-Pacific Entomology 6: 15. Holotype ${ }^{*}$, Taiwan, Ilan Co., Taiheisan [Taipingshan], 9 May 1942, S. Issiki. Specimen not found. [Lecithoceridae]

taiwana Park \& Hodges, 1995 (Dichomeris); Insecta Koreana 12: 48. Holotype ${ }^{*}$, Taiwan, Nantou Co., Sunmoon Lake, 760 m, 20-25 June 1980, D. Davis. USNM Genitalia Slide No. 87329. [Gelechiidae: Dichomeridinae]

taiwana Park \& Wu, 2003 (Autosticha); Insecta Koreana 20: 213. Holotype ơ, Taiwan, Nantou Co., Lushan, ca. 30 km W Wu-she, ca. 1000 m, 27-31 May 1980, D. Davis. Specimen not found. [Autostichidae: Autostichinae]

tanyglocha Wu \& Park, 1999 (Hyperochtha); Tinea 16: 69. Holotype ${ }^{*}$, Sri Lanka, Kan. District, Kandy, Udawattakele, 26-28 May 1975, Messersmith, Williams \& Karunaratne. [Lecithoceridae]

tarmes Hodges, 1999 (Chionodes); Moths of America north of Mexico, fasc. 7.6: 66. Holotype ${ }^{\star}$, USA, Michigan, Allegheny Co., T2N R14W S31, 1 August 1986, G. Balogh. [Gelechiidae: Gelechiinae] 
taurusella Adamski, 2004 (Blastobasis); Holarctic Lepidoptera 7: 51. Holotype ${ }^{*}$, USA, 510 Texas, Brownsville, 4 May 1904, H. S. Barber. USNM Genitalia Slide No. 81275. [Coleophoridae: Blastobasinae]

tehuacana Busck, 1913 (Gelechia); Insecutor Inscitiae Menstruus 1: 140, Holotype ox, Mexico, Tehuacan, July, R. Müller. USNM Type No. 16661, Genitalia Slide AB Aug. 6, 1936. [Gelechiidae: Gelechiinae]

telerella Kaila, 1999 (Elachista); Acta Zoologica Fennica 211: 64. Holotype o*, USA, Oregon, Wallowa Co., Wallowa Lake, 6 June 1970, J. F. G. Clarke. USNM Genitalia Slide No. 82499. [Elachistidae: Elachistinae]

telosperma Diakonoff, 1968 (Lecithocera); Bulletin of the U.S. National Museum 257 (1967): 143. Holotype $\sigma^{*}$, Philippine Islands, Luzon, San Miguel, Tarlac, 1-21 October 1945, J. G. Franclemont. USNM Gentialia Slide No. 89971 [Lecithoceridae]

tenuicornis Clarke, 1942 (Arla); Proceedings of the U.S. National Museum 92: 269. Holotype $o^{\star}$, USA, Washington, Klickitat Co., Warwick, 9 June 1931, T. M. Clarke. USNM Type No. 56269, USNM Genitalia Slide No. 7542. Right fore- and hindwing lacking. [Gelechiidae: Gelechiinae]

terinella Barnes \& Busck, 1920 (Agonopteryx); Contributions to the Natural History of the Lepidoptera of North America 4: 232. Holotype *, USA, Colorado, Silverton, 24-30 August. USNM Type No. 22667, USNM Genitalia Slide Nos. 193. [Elachistidae: Depressariinae]

terminalis Clarke, 1951 (Coptotelia); Acta Zoologica Lilloana 11: 348. Holotype + , Mexico, Tabasco, Teapa, 13 December, R. Müller. USNM Type No. 61120, USNM Genitalia Slide No. 69422. [Oecophoridae: Oecophorinae]

terminimaculella Kearfott, 1908 (Gelechia); Journal of the New York Entomological Society 16: 184. Holotype $\sigma^{\star}$, Canada, Manitoba, Aweme, 13-16 June, N. Criddle. [Gelechiidae: Gelechiinae]

terracottella Busck, 1900 (Gnorimoschema); Proceedings of the U.S. National Museum 23: 227. Holotype + , USA, Florida, Palm Beach, r.f. Iva imbricata, em. March, H. Dyar. USNM Type No. 4934, USNM Genitalia Slide No. 10647. [Gelechiidae: Gelechiinae]

terrenella Busck, 1914 (Anacampsis); Proceedings of the U.S. National Museum 47: 10. Holotype $\sigma^{\star}$, Panama, Porto Bello, May, A. Busck. USNM Type No. 16676. [Gelechiidae: Gelechiinae] 
tessa Clarke, 1947 (Chionodes); Journal of the Washington Academy of Sciences 37: 246.

Holotype $\sigma^{x}$, California, Sonoma Co., Petaluma, [no date], E. C. Johnston. USNM Type

No. 58237, USNM Genitalia Slide No. 8029. [Gelechiidae: Gelechiinae]

testor Hodges, 1966 (Batrachedra); Transactions of the American Entomological Society 92: 605. Holotype ${ }^{*}$, USA, Florida, Fisheating Creek, near Palmdale, 7-10 May 1964, R. W. Hodges. USNM Type No. 67855, USNM Genitalia Slide No. 5064. [Batrachedridae]

tetradymiella Busck, 1903 (Gnorimoschema); Proceedings of the U.S. National Museum 25: 834. Holotype (uncertain sex), USA, California, Los Angeles. Specimen not found. [Gelechiidae: Gelechiinae]

tetraphyta Meyrick, 1931 (Pyramidobela); Exotic Microlepidoptera 4: 174. Lectotype $o^{x}$, Mexico, Hidalgo, Real del Monte, Van Ostrand. USNM Genitalia Slide No. 664. Designated by Powell (1973). [Elachistidae: Ethmiinae]

thaihesiana Park, 1999 (Lecithocera); Zoological Studies 38: 246. Holotype ${ }^{*}$, Taiwan, Ilan Co., Taiheisan (=Taipingshan), 10 May 1942, S. Issiki. USNM Genitalia Slide No. 82498. [Lecithoceridae]

thaliae Hodges, 1966 (Lita); Proceedings of the U.S. National Museum 119: 28. Holotype ox, USA, Utah, Eureka, 16 August 1911, T. Spalding. [Gelechiidae: Gelechiinae]

thamnocephala Clarke, 1978 (Eraina); Smithsonian Contributions to Zoology 273: 3. Holotype $\diamond^{\star}$, Chile, Maule, Pelluhue, 600 m, 2 December 1953, L. E. Peña. USNM Type No. 73690, USNM Genitalia Slide No. 24137. [Oecophoridae: Oecophorinae]

thapsina Wu \& Park, 1999 (Frisilia); Korean Journal of Systematic Zoology 15: 2. Holotype ${ }^{\star}$, Sri Lanka, Kal. Dist, Agalawatta, 13-14 October 1976, black light, Hevel, Dietz, Karunaratne \& Balasooriya. USNM Genitalia Slide No. 88825. [Lecithoceridae]

theca Clarke, 1957 (Batrachedra); Acta Zoologica Mexicana 2: 2. Holotype + , Mexico, Distrito Federal, Santa Rosa Ameyalco, r.f. Agave, 28 February 1954. USNM Type No. 63431, USNM Genitalia Slide No. 5115. [Batrachedridae]

thelmae Clarke, 1941 (Agonopterix); Proceedings of the U.S. National Museum 90: 96. Holotype $\sigma^{\star}$, USA, Maryland, Montgomery Co., Plummers Island, Potomac River, em. 2 August 1920, H. Barber. USNM Type No. 53175, USNM Genitalia Slide No. 241. [Elachistidae: Depressariinae] 
thenara Wu, 2001 (Eccedoxa); Acta Zootaxonomia Sinica 26: 206. Holotype ơ, Sri Lanka, 510 Rat. District, Uggalkaltota, Irrigation Bungalow, 350', 31 January-8 February 1970, D. Davis \& B. Rowe. [Lecithoceridae]

theobromae Busck, 1920 (Zetesima); Insecutor Inscitiae Menstruus 8: 88. Holotype $\sigma^{x}$, Dutch Guiana, Paramaribo, Quaintance No. 9231, r.f. cacao, A. Reyne. USNM Type No. 22324, USNM Genitalia Slide No. 13609. [Elachistidae: Stenomatinae]

theurgis Hodges, 1999 (Chionodes); Moths of America north of Mexico, fasc. 7.6: 213. Holotype $\sigma^{\star}$, USA, Colorado, Chaffe Co., 4 mi SW Buena Vista, 8700', 10 July 1982, R. W. Hodges. [Gelechiidae: Gelechiinae]

thomasi Barnes \& Busck, 1920 (Stenoma); Contributions to the Natural History of the Lepidoptera of North America 4: 240. Holotype ${ }^{*}$, USA, Arizona, Palmerlee [no date]. USNM Genitalia Slide No. 82506. The name on both the specimen and slide label is "Stenoma thomasiella." [Elachistidae: Stenomatinae]

thoristes Busck, 1911 (Stenoma); Proceedings of the U.S. National Museum 40: 220. Holotype $\sigma^{\star}$, French Guiana, St. Jean, Maroni River, [no date], W. Schaus. USNM Type No. 13582, USNM Genitalia Slide No. 13990. [Elachistidae: Stenomatinae]

thujaella Kearfott, 1903 (Recurvaria); Journal of the New York Entomological Society 11: 154. Holotype $\sigma^{*}$, USA, r.f. Thuja occidentalis. According to the original description, this species was described from 42 specimens reared from Thuja occidentalis; it clearly states that the "type" is in USNM. Abdomen lacking. [Gelechiidae: Gelechiinae]

thustra Clarke, 1947 (Depressaria); Journal of the Washington Academy of Sciences 37: 15. Holotype $\diamond^{*}$ USA, Washington, Klickitat Co., Gilmer, 26 May 1946, r.f. Lomatium triternatum macrocarpum, J. F. G. Clarke. USNM Type No. 58009, USNM Genitalia Slide No. 650. [Elachistidae: Depressariinae]

thyotes Hodges, 1999 (Chionodes); Moths of America north of Mexico, fasc. 7.6: 96. Holotype + , USA, Texas, Cameron Co., Southmost, 27 October 1986, E. C. Knudson. USNM Genitalia Slide No. 964. [Gelechiidae: Gelechiinae]

timarella Landry \& Wright, 1993 (Coleophora); Canadian Entomologist 125: 578. Holotype ${ }^{\star}$, Arizona, Coconino Co., West Fork, 16 mi SW Flagstaff, 6500', 5 August 1961, R. W. Hodges. USNM Genitalia Slide No. 87223. [Coleophoridae: Coleophorinae] 
tistra Hodges, 1974 (Decantha), Moths of America north of Mexico, fasc. 6.2: 107. Holotype $\sigma^{*}$, USA, Arizona, Coconino Co., Fort Valley, 7.5 mi N Flagstaff, 28 June 1961, R. W. Hodges. USNM Genitalia Slide No. 5795. [Oecophoridae: Oecophorinae]

titanica Walsingham, 1912 (Holcocera); Biologia Centrali-Americana, Lepidoptera Heterocera 4: 151. Holotype 9 , Mexico, Oaxaca, Salina Cruz, July 1906, W. Schaus. USNM Type No. 81038, USNM Genitalia Slide No. 81038. [Coleophoridae: Blastobasinae]

tolmeta Walsingham, 1912 (Stenoma); Biologia Centrali-Americana, Lepidoptera Heterocera 4: 166. Holotype 9 , Panama, Canal Zone, Tabernilla, June 1907, A. Busck. Abdomen lacking. [Elachistidae: Stenomatinae]

tortricella Chambers, 1874 (Stenoma); Canadian Entomologist 6: 235. Holotype ${ }^{*}$, USA, Texas, Waco [no date]. USNM Type No. 433, Genitalia Slide AB Mar. 22, 1920. [Elachistidae: Stenomatinae]

tortriciformella Clemens, 1860 (Menesta); Proceedings of the Academy of Natural Sciences, Philadelphia 1860: 213. Holotype $q$, USA, “100.” USNM Type No. 7352, W. D. Duckworth Genitalia Slide No. 3001. [Elachistidae: Stenomatinae]

trachycantha Clarke, 1978 (Doina); Smithsonian Contributions to Zoology 273: 33. Holotype $\sigma^{\star}$, Chile, Centro-Austral, January-March 1898, V. Izquerdo. USNM Type No. 73709, USNM Genitalia Slide No. 24264. [Amphisbatidae]

traditionis Clarke, 1950 (Euchionodes); Journal of the Washington Academy of Sciences 40: 285. Holotype $\sigma^{*}$, Argentina, Tigre, May 1941, F. Bourquin. USNM Type No. 59422 , USNM Genitalia Slide No. 87552. [Gelechiidae: Gelechiinae]

traducella Busck, 1914 (Gelechia); Proceedings of the U.S. National Museum 47: 12. Holotype + , Panama, La Chorrera, May, A. Busck. USNM Type No. 15826, J. F. G. Clarke Genitalia Slide No. 10978. Specimen lacking all wings except left hindwing. [Gelechiidae: Gelechiinae]

transversa Clarke, 1971 (Gonionota); Smithsonian Contributions to Zoology 95: 25. Holotype $o^{*}$, Brazil, Paraná, Castro, [no date], W. Schaus. USNM Type No. 71097, USNM Genitalia Slide No. 68765. [Oecophoridae: Oecophorinae]

transversella Busck, 1914 (Ethmia); Insecutor Inscitiae Menstruus 2: 53. Holotype $o^{*}$, Costa Rica, Juan Viñas, [no date], W. Schaus. USNM Type No. 18270, USNM Genitalia Slide No. 98449. [Elachistidae: Ethmiinae] 

USNM Genitalia Slide No. 14116. [Elachistidae: Stenomatinae]

triangulella Busck, 1907 (Gelechia); Proceedings of the Entomological Society of Washington 8: 91. Syntypes (6), USA, Arizona, Bright Angel and Williams, June \& July, H. S. Barber. USNM Type No. 9894. [Gelechiidae: Gelechiinae]

triangulifera Kaila, 1997 (Elachista); Acta Zoologica Fennica 206: 30. Holotype ${ }^{*}$, USA, Oregon, Cook Co., Paulina, 19 May 1965, J. H. Baker. USNM Genitalia Slide No. 89300. [Elachistidae: Elachistinae]

triannulata Clarke, 1958 (Paralida); Entomological News 69: 2. Holotype $\sigma^{*}$, Japan, Honshu, Kinki, Ikeda, r.f. Melia azedarach var. japonica, em. 16 September 1949, S. Issiki. USNM Type No. 63586, J. F. G. Clarke Genitalia Slide No. 10612. Right fore- and hindwing lacking. [Gelechiidae: Gelechiinae]

tribrachia Park, 2001 (Carodista); in Park \& Wu, Insecta Koreana 18: 146. Holotype $\sigma^{\star}$, Sri Lanka, Gal. District, Kanneliya, 200', 15-17 October 1975, G. Hevel, R. Dietz, S. Karunaratne \& D. Balassoorilya. USNM Genitalia Slide No. 10668. [Lecithoceridae]

trichella Busck, 1908 (Batrachedra); Canadian Entomologist 40: 196. Holotype ox, USA, Pennsylvania, Oak Station, 13 June 1907, F. Marloff. USNM Type No. 11562, USNM Genitalia Slide No. 5192. [Batrachedridae]

trichella Busck, 1914 (Parastega); Proceedings of the U.S. National Museum 47: 16. Holotype ${ }^{\star}$, Panama, Porto Bello, April 1912, A. Busck. USNM Type No. 15832, USNM Genitalia Slide No. 10668. [Gelechiidae: Gelechiinae]

trichogramma Clarke, 1986 (Asymphorodes); Smithsonian Contributions to Zoology 416: 259. Holotype $\sigma^{*}$, Marquesas Archipelago, Hiva Oa, Atuona, 25 February 1968, J. F. G. \& T. M. Clarke. USNM Type No. 100814. [Cosmopterigidae: Cosmopteriginae]

trico Hodges, 1999 (Chionodes); Moths of America north of Mexico, fasc. 7.6: 211. Holotype $\sigma^{\star}$, USA, South Dakota, Lawrence Co., Hardy Work Center, T3N R1E S30, 21 July 1965, R. Hodges. [Gelechiidae: Gelechiinae]

tricristatella Chambers, 1875 (Leucophryne); Canadian Entomologist 7: 211. Syntype $q$, Canada [no other data]. USNM Type No. 5773. [Coleophoridae: Momphinae] 
tricrosura Wu \& Park, 1999 (Frisilia); Korean Journal of Systematic Zoology 15: 3. Holotype ${ }^{\star}$, Sri Lanka, Gal. District, Kanneliya, 200', 15-17 October 1976, black light, G. Hevel, R. Dietz, S. Karunaratne \& D. Balasooriya. USNM Genitalia Slide No. 88821. [Lecithoceridae]

tridentata Clarke, 1947 (Filatima); Journal of the Washington Academy of Sciences 37: 266. Holotype $\sigma^{*}$, USA, California, Riverside Co., Snow Creek, r.f. Salix, em. 21 October 1938, C. M. Dammers. Specimen not found. [Gelechiidae: Gelechiinae]

trigrapha Clarke, 1986 (Asymphorodes); Smithsonian Contributions to Zoology 416: 189. Holotype $\sigma^{*}$, Marquesas Archipelago, Nuku Hiva, Tunoa Ridge, 23 January 1968, J. F. G. \& T. M. Clarke. USNM Type No. 100776, USNM Genitalia Slide No. 24854. [Cosmopterigidae: Cosmopteriginae]

trilobella Park \& Hodges, 1995 (Dichomeris); Insecta Koreana 12: 42. Holotype ${ }^{\star}$, Taiwan, Pingtung Co., Kenting Botanical Garden, 22-25 July 1980, D. Davis. USNM Genitalia Slide No. 87323. [Gelechiidae: Dichomeridinae]

trimacula Clarke, 1978 (Atha); Smithsonian Contributions to Zoology 273: 57. Holotype ox, Chile, Llanquihue, Peulla, 9 March 1959, J. F. G. Clarke. USNM Type No. 73722, USNM Genitalia Slide No. 24286. Right fore- and hindwing lacking. [Oecophoridae: Oecophorinae]

trimaculella Chambers, 1874 (Gelechia); Canadian Entomologist 6: 238. Lectotype o*, USA, Texas, Waco. USNM Type No. 470. Designated by Hodges (1986). Left fore- and hindwing lacking [Gelechiidae: Dichomeridinae]

trinota Clarke, 1965 (Echinoglossa); Proceedings of the U.S. National Museum 117: 83. Syntype $q$, Chile, Juan Fernandez Islands. Clarke (1965) inadvertently failed to designate a holotype or list the specimens examined. [Gelechiidae: Gelechiinae]

trinotella Busck, 1906 (Trichotaphe); Canadian Entomologist 32: 122. Holotype 9 , USA, Pennsylvania, Pittsburgh, 29 May 1905, H. Engel. USNM Type No. 9794, USNM Genitalia Slide No. 9244. Homonym, replaced by Dichomeris agonia Hodges (1986). [Gelechiidae: Dichomeridinae]

trinotella Coquillet, 1883 (Nothris); Papilio 3: 81. Holotype (uncertain sex), USA, Illinois, "1052," Fernald Collection. Type consists of head (with most features in tact), prothorax, mesothorax, and right forewing. [Gelechiidae: Dichomeridinae]

GELECHIOIDEA TYPES IN USNM

(C) 2004 Magnolia Press 

[Gelechiidae: Dichomeridinae]

trizeugma Wu \& Park, 1999 (Frisilia); Korean Journal of Systematic Zoology 15: 3. Holotype $\sigma^{*}$, Sri Lanka, Kan. District, Teldeniya 1400', 18-20 November 1976, blacklight, G. Hevel, R. Dietz, S. Karunaratne \& D. Balasooriya. USNM Genitalia Slide No. 88828. [Lecithoceridae]

trochilosticta Walsingham, 1913 (Stenoma); Biologia Centrali-Americana, Lepidoptera Heterocera 4: 177. Holotype $o^{*}$, Panama, Chiriqui, [no date], W. Schaus, Walsingham Collection, 1910-427. USNM Genitalia Slide No. 13400. [Elachistidae: Stenomatinae]

trophella Busck, 1903 (Gelechia); Proceedings of the U.S. National Museum 25: 860. Lectotype ${ }^{x}$, USA, Colorado, Platte Canyon, [no date], Dyar. USNM Type No. 6375, USNM Genitalia Slide No. 8339. Designated by Hodges (1999). [Gelechiidae: Gelechiinae]

truncata Clarke, 1978 (Doina); Smithsonian Contributions to Zoology 273: 27. Holotype $\sigma^{\star}$, Chile, Centro-Austral, January-March 1898, V. Izquerdo. USNM Type No. 73704, USNM Genitalia Slide No. 24256. [Amphisbatidae]

tryphon Busck, 1920 (Stenoma); Insecutor Inscitiae Menstruus 8: 89. Holotype $\sigma^{\star}$, Guatemala, Cayuga, [no date], W. Schaus. USNM Type No. 22308, USNM Genitalia Slide No. 13222. [Elachistidae: Stenomatinae]

tuberosella Busck, 1931 (Gnorimoschema); Proceedings of the Entomological Society of Washington 33: 59. Syntypes (uncertain sex), Peru, Lima, r.f. Solanum tuberosa, [no date], C. H. T. Townsend. USNM Type No. 43309. [Gelechiidae: Gelechiinae]

tunicata Busck, 1914 (Cryptolechia); Proceedings of the U.S. National Museum 47: 29. Holotype + , Panama, Porto Bello, June 1912, A. Busck. USNM Type No. 16749, USNM Genitalia Slide No. 69395. [Elachistidae: Depressariinae]

umbrimarginella Busck, 1907 (Ethmia); Proceedings of the Entomological Society of Washington 8: 94. Holotype $\sigma^{x}$, USA, New Mexico, Las Cruces, Mesilla Park, r.f. Rumex hymenosepalus, T. D. A. Cockerell. USNM Type No. 9899, USNM Genitalia Slide No. 98446. [Elachistidae: Ethmiinae] 
ungulatella Busck, 1914 (Ethmia); Proceedings of the U.S. National Museum 47: 34. Holotype + , Panama, Cabima, May 1911, A. Busck. USNM Type No. 16696. [Elachistidae: Ethmiinae]

unifasciella Busck, 1903 (Gelechia); Proceedings of the U.S. National Museum 25: 865. Holotype $\sigma^{*}$, USA, Arizona, Williams, July, Barber. USNM Type No. 6380, USNM Genitalia Slide No. 9983. [Gelechiidae: Gelechiinae]

unistrigella Busck, 1914 (Anacampsis); Proceedings of the U.S. National Museum 47: 9. Holotype $\sigma^{\star}$, Panama, Taboga Island, June, A. Busck. USNM Type No. 15821. [Gelechiidae: Gelechiinae]

untomiella Busck, 1906 (Untomia); Proceedings of the U.S. National Museum 30: 727. Holotype $\sigma^{*}$, USA, Texas, Brownsville, June, H. S. Barber. USNM Type No. 9771, USNM Genitalia Slide No. 10651. [Gelechiidae: Gelechiinae]

ursula Walsingham, 1910 (Anacampsis); Biologia Centrali-Americana, Lepidoptera Heterocera 4: 35. Syntype 9 , Mexico, Morelos, Cuernavaca, June, H. H. Smith. Walsingham identified a "type $\sigma^{\star}$ " and "type $q$ " for this species. [Gelechiidae: Gelechiinae]

ustor Hodges, 1999 (Chionodes); Moths of America north of Mexico, fasc. 7.6: 207. Holotype $\sigma^{*}$, USA, Wyoming, Sublette Co., Wind River Mountains, Bridger Forest Campground, 10 August 1972, R. Leuschner. USNM Genitalia Slide No. 945. [Gelechiidae: Gelechiinae]

utricula Wu \& Park, 1998 (Hygroplasta); Insecta Koreana 15: 10. Holotype ${ }^{*}$, Sri Lanka, Kan. District, $5 \mathrm{mi}$ NW Mahiyangana, malaise trap at Hasalaka Irrigation Bungalow, 30 March-9 April 1971, P. \& P. Spangler. USNM Genitalia Slide No. 88945. [Lecithoceridae]

vacciniella Busck, 1915 (Dichomeris); Proceedings of the Entomological Society of Washington 17: 83. Lectotype ${ }^{*}$, USA, New Jersey, Princeton, bred, "7-9-14," Quaintance no. 11934, H. B. Scammell. The specimen is represented by the head and prothorax only. Designated by Hodges (1986). [Gelechiidae: Dichomeridinae]

vaccinii Clarke, 1947 (Filatima); Journal of the Washington Academy of Sciences 37: 270. Holotype $\sigma^{\star}$, USA, New Jersey, Toms River, r.f. Vaccinium vacillans, em. 30 June 1923, H. B. Scammell. USNM Type No. 58254, USNM Genitalia Slide No. 9701. [Gelechiidae: Gelechiinae] 
vaniae Clarke, 1947 (Filatima); Journal of the Washington Academy of Sciences 37: 266. Holotype $\sigma^{*}$, USA, Utah, Murray, r.f. Ribes, July 1913, P. H. Timberlake. USNM Type No. 58255, USNM Genitalia Slide No. 9714. [Gelechiidae: Gelechiinae]

vanis Busck, 1911 (Stenoma); Proceedings of the U.S. National Museum 40: 221. Holotype $\sigma^{*}$, French Guiana, St. Jean, Maroni River, [no date], W. Schaus. USNM Type No. 13584, USNM Genitalia Slide No. 13021. [Elachistidae: Stenomatinae]

variabilis Busck, 1903 (Gelechia); Proceedings of the U.S. National Museum 25: 871. Syntypes $\left(20^{x}, 1 \%\right)$, USA, California, Placer Co., August. USNM Type No. 6385. Described from 20 syntypes. [Gelechiidae: Gelechiinae]

varronia Busck, 1913 (Dichomeris); Insecutor Inscitiae Menstruus 1: 89. Holotype $q$, British Guiana, Pln. Kitty, r.f. blacksage (Varronia curissavaca), [no date], H. Moore. USNM Type No. 16012. Abdomen in gelatin capsule. [Gelechiidae: Dichomeridinae]

velatella Busck, 1907 (Telphusa); Proceedings of the Entomological Society of Washington 8: 90 . Syntypes $\left(10^{\star}, 2 \circ\right)$, USA, Arizona, Williams, July, H. S. Barber. USNM Type No. 9893, USNM Slide No. $6679\left({ }^{\star}\right)$. Abdomen lacking from one female. [Gelechiidae: Gelechiinae]

veledae Hodges, 1966 (Lita); Proceedings of the U.S. National Museum 119: 24. Holotype ๙, USA, California, Imperial Co., Dixieland, 15-30 March 1922, O. C. Poling. USNM Type No. 67648, USNM Genitalia Slide No. 10673. [Gelechiidae: Gelechiinae]

veles Hodges, 1999 (Chionodes); Moths of America north of Mexico, fasc. 7.6: 212. Holotype $\sigma^{*}$, USA, Colorado, Chaffee Co., Castles, 8 mi E Buena Vista, 8800', 11 July 1982, R. W. Hodges. [Gelechiidae: Gelechiinae]

venatum Busck, 1911 (Stenoma); Proceedings of the U.S. National Museum 40: 217. Holotype $\$$, French Guiana, St. Jean, Maroni River, [no date], W. Schaus. USNM Type No. 13576, USNM Genitalia Slide No. 12873. [Elachistidae: Stenomatinae]

venifica Hodges, 1964 (Aeaea); Proceedings of the U.S. National Museum 115: 301. Holotype ${ }^{\star}$, USA, Illinois, Putnam Co., 26 June 1957, M. O. Glenn. USNM Genitalia Slide No. 4487. [Cosmopterigidae: Chrysopeleiinae]

venis Hodges, 1978 (Anoncia); Moths of America north of Mexico, fasc. 6.1: 70. Holotype $\uparrow$, Arizona, Yuma Co., Quartzite, 4 April 1973, J. H. Baker. USNM Genitalia Slide No. 6093. [Cosmopterigidae: Cosmopteriginae] 
venosa Busck, 1914 (Galtica); Proceedings of the U.S. National Museum 47: 6. Holotype o, Panama, Porto Bello, April, A. Busck. USNM Type No. 15817. Right fore- and hindwing and abdomen lacking. [Gelechiidae: Gelechiinae]

ventralilata Wu \& Park, 1999 (Torodora); Tinea 16: 63. Holotype o*, Sri Lanka, Gal. District, $4 \mathrm{mi}$ E Udugama, Kanneliya Jungle, malaise trap, 7 October 1973, C. Krombein, Karunarata, Fernando \& Ferdinando. USNM Genitalia Slide No. 89205. [Lecithoceridae]

ventrellus Fitch, 1854 (Chaetochilus); Transactions of the New York State Agricultural Society 13: 234. Holotype ${ }^{\star}$, USA, New York, [no date]. USNM Genitalia Slide No. 10641. [Gelechiidae: Dichomeridinae]

vestaliella Dietz, 1910 (Holcocera); Transactions of the American Entomological Society 36: 63. Lectotype ${ }^{*}$, USA, Maryland, Montgomery Co., Plummers Island, June 1903, A. Busck. USNM Genitalia Slide No. 11140. Designated by Adamski \& Hodges (1996). [Coleophoridae: Blastobasinae]

vestita Walsingham, 1911 (Glyphidocera); Biologia Centrali-Americana, Lepidoptera Heterocera 4: 112. Holotype $\sigma^{\star}$, Panama, Tabernilla, Canal Zone, [no date], A. Busck. Abdomen lacking. [Glyphidoceridae]

vibex Hodges, 1978 (Melanocinclis); Moths of America north of Mexico, fasc. 6.1: 43. Holotype ${ }^{\star}$, USA, Florida, Archbold Biological Station, Lake Placid, 16-22 May 1964, R. W. Hodges. USNM Type No. 70569, USNM Genitalia Slide No. 5586. [Cosmopterigidae: Cosmopteriginae]

victor Hodges, 1964 (Aeaea); Proceedings of the U.S. National Museum 115: 304. Holotype $o^{\star}$, USA, Virginia, Cherrydale, Hopkins No. 13942g, r.f. oak, 22 September 1922, A. Busck. USNM Genitalia Slide No. 4552. [Cosmopterigidae: Chrysopeleiinae]

villella Busck, 1900 (Triclonella); Journal of the New York Entomological Society 8: 238. Holotype $\diamond^{\star}$, USA, Maryland, Montgomery Co., Cabin John Bridge, r.f. Xilisma (Andromeda) ligustrina?, em. 11 May 1900. USNM Type No. 5355, USNM Genitalia Slide No. 81572. Left forewing and right hindwing lacking. [Coleophoridae: Blastobasinae]

villella Busck, 1904 (Cosmopteryx); Proceedings of the U.S. National Museum 27: 768. Holotype 9 , USA, Washington, Seattle, 7 June 1902, T. Kincaid. USNM Type No. 7866, USNM Genitalia Slide No. 4987. [Elachistidae: Agonoxenidae] 

Slide No. 9454. [Gelechiidae: Dichomeridinae]

violacea Busck, 1914 (Atoponeura); Proceedings of the U.S. National Museum 47: 4. Holotype $\sigma^{*}$ Panama, Trinidad River, March, A. Busck. USNM Type No. 15816, J. F. G. Clarke Genitalia Slide No. 10976. [Gelechiidae: Gelechiinae]

virgea Clarke, 1947 (Filatima); Journal of the Washington Academy of Sciences 37: 272. Holotype $o^{\star}$, USA, Texas, Presidio, r.f. Acacia vernicosa, em. 8 November 1944, J. H. Russell. USNM Type No. 58258, USNM Genitalia Slide No. 9559. [Gelechiidae: Gelechiinae]

virginia Busck, 1914 (Doxa); Proceedings of the U.S. National Museum 47: 27. Holotype o, Panama, Trinidad River, March 1912, A. Busck. USNM Type No. 16745. [Oecophoridae: Oecophorinae]

viridis Busck, 1914 (Stenoma); Proceedings of the U.S. National Museum 47: 42. Holotype 9 , Panama, Trinidad River, March 1912, A. Busck. USNM Type No. 16716, USNM Genitalia Slide No. 12874. [Elachistidae: Stenomatinae]

vita Busck, 1911 (Gonioterma); Proceedings of the U.S. National Museum 40: 226. Holotype $\uparrow$, French Guiana, St. Jean, Maroni River, [no date], W. Schaus. USNM Type No. 13595, USNM Genitalia Slide No. 14011. [Elachistidae: Stenomatinae]

vittella Busck, 1916 (Duvita); Proceedings of the Entomological Society of Washington 18: 147. Holotype, USA, New York, Long Island, r.f. pine cones, [no date. Heinrick. USNM Type No. 20206. [Gelechiidae: Gelechiinae]

vivax Busck, 1914 (Stenoma); Proceedings of the U.S. National Museum 47: 40. Holotype \%, Panama, Cabima, May 1911, A. Busck. USNM Type No. 16711, Genitalia Slide AB July 12, 1931. [Elachistidae: Stenomatinae]

vividella Busck, 1914 (Stenoma); Proceedings of the U.S. National Museum 47: 43. Holotype $\sigma^{\star}$, Panama, La Chorrera, March 1912, A. Busck. USNM Type No. 16718, USNM Genitalia Slide No. 13238. [Elachistidae: Stenomatinae]

volcanella Powell, 1973 (Ethmia); Smithsonian Contributions to Zoology 120: 97. Holotype ${ }^{\star}$, Mexico, Oaxaca, Tehuantepec, 15-16 July 1964, P. J. Spangler. USNM Genitalia Slide No. 87685. [Elachistidae: Ethmiinae] 
volo Hodges, 1999 (Chionodes); Moths of America north of Mexico, fasc. 7.6: 75. Holotype ${ }^{\star}$, USA, Texas, Jeff Davis Co., Fort Davis, 5000', 18 May 1971, A. \& M. E. Blanchard. USNM Genitalia Slide No. 15207. [Gelechiidae: Gelechiinae]

walsinghamella Beutenmüller, 1889 (Psecadia); Entomologica Americana 5: 9. Holotype $\uparrow$, USA, West Virginia, [no date], Beutenmüller. USNM Type No. 439. Left forewing lacking. [Elachistidae: Ethmiinae]

walsinghami Dietz, 1900 (Pseudochelaria); Entomological News 11: 352. Syntypes (6), USA, Pennsylvania, Hazleton, 7 June 1897. [Gelechiidae: Gelechiinae]

washingtoniella Busck, 1904 (Gnorimoschema); Proceedings of the U.S. National Museum 27: 756. Holotype ${ }^{*}$, USA, Washington, Pullman, 16 August 1898, C. V. Piper. USNM Type No. 7856, USNM Genitalia Slide No. 7045, USNM Wing Slide No. 7046. [Gelechiidae: Gelechiinae]

washingtoniella Busck, 1906 (Trichotaphe); Canadian Entomologist 38: 121. Lectotype o*, USA, Washington, D.C., r.f. Eupatorium, em. 1 June 1902, A. Busck. USNM Type No. 9793. Designated by Hodges (1986). Abdomen lacking. [Gelechiidae: Dichomeridinae]

websteri Clarke, 1942 (Aroga); Proceedings of the U.S. National Museum 92: 274. Holotype $\sigma^{*}$, USA, Washington, Pullman, 18 July 1930, J. F. G. Clarke. USNM Type No. 56275, USNM Genitalia Slide No. 9984. [Gelechiidae: Gelechiinae]

whalleyi Duckworth, 1967 (Thioscelis); Proceedings of the U.S. National Museum 123: 5. Holotype $\sigma^{*}$, Brazil, Campo Bella Rio, 30 January 1929. [Elachistidae: Stenomatinae]

whitmanella Clarke, 1942 (Chionodes); Proceedings of the U.S. National Museum 92: 271. Holotype ${ }^{*}$, USA, Washington, Whitman Co., Pullman, 23 September 1932, J. F. G. Clarke. USNM Type No. 56271, USNM Genitalia Slide No. 7947. [Gelechiidae: Gelechiinae]

whitmani Clarke, 1941 (Depressaria); Proceedings of the U.S. National Museum 90: 182. Holotype $o^{*}$, USA, Washington, Whitman Co., Snake River, opposite Clarkston, 23 May 1935, r.f. Lomatium macrocarpum, J. F. G. Clarke. USNM Type No. 52083, USNM Genitalia Slide No. 565. [Elachistidae: Depressariinae]

wilpattuae Park, 2001 (Carodista); in Park \& Wu, Insecta Koreana 18: 148. Holotype ${ }^{\star}$, Sri Lanka, Anu. District, Wildlife Soc. Bungalo, Hunuwilagama, 200', 10-19 March 1970, D. Davis \& B. Rowe. USNM Genitalia Slide No. 92166. [Lecithoceridae] 
wrightorum Adamski \& Metzler, 2000 (Glyphidocera); Proceedings of the Entomological Society of Washington 102: 302. Holotype ${ }^{*}$, USA, Ohio, Greene Co., Bath Township, Wright-Patterson Air Force Base, Huffman Prairie, G-4, 39² $48.4^{\prime} \mathrm{N}, 84^{\circ} 3.5^{\prime} \mathrm{W}, 14$ July 1995, malaise trap, E. Metzler. USNM Genitalia Slide No. 81650. [Glyphidoceridae]

wyattella Barnes \& Busck, 1920 (Stagmatophora); Contributions to the Natural History of the Lepidoptera of North America 4: 222. Holotype ${ }^{*}$, USA, Illinois, Palos Park, 15 July 1911, A. Wyatt. USNM Genitalia Slide No. 12412. [Cosmopterigidae: Cosmopteriginae]

xanthoa Hodges, 1986 (Dichomeris); Moths of America north of Mexico, fasc. 7.1: 102. Holotype $\propto^{*}$, USA, Nebraska, Cherry Co., Fort Niobrara National Wildlife Refuge, 19 June 1983, R. W. Hodges. USNM Genitalia Slide No. 12112. [Gelechiidae: Dichomeridinae]

xanthophilella Barnes \& Busck, 1920 (Gelechia); Contributions to the Natural History of the Lepidoptera North America 4: 228. Holotype 9 , California, Inyo Co., Olancha, 8-15 June. USNM Genitalia Slide No. 8187. [Gelechiidae: Gelechiinae]

xanthosoma Dognin, 1913 (Stenoma); Annales de la Société Entomologique de Belgique 57: 416. Holotype $\sigma^{\star}$, French Guiana, St. Laurent, Maroni River, Dognin Collection. USNM Type No. 32362, USNM Genitalia Slide No. 14031. [Elachistidae: Stenomatinae]

xanthotarsa Becker, 1982 (Timocratica); Bulletin of the British Museum (Natural History), Entomology 45: 242. Holotype ox, Panama, Barro Colorado Island, 1-9 May 1964, W. D. \& S. S. Duckworth. Left hindleg in gelatin capsule. [Elachistidae: Stenomatinae]

xenodora Clarke, 1982 (Odonna); Journal of Research on the Lepidoptera 20: 49. Holotype ${ }^{*}$, Colombia, Cauca, Paramo de Parace, Lake San Rafael, 3570 m, 29 January 1959, J. F. G. Clarke. USNM Type No. 100176, USNM Genitalia Slide No. 25135. Right foreand hindwing lacking. [Oecophoridae: Oecophorinae]

xestocephala Diakonoff, 1968 (Canthonistis); Bulletin of the U.S. National Museum 257 (1967): 127. Holotype $\sigma^{*}$, Philippine Islands, Luzon, Benguet Subprovince, Baguio, [no date], P. J. Baker. USNM Genitalia Slide No. 89998. [Gelechiidae: Gelechiinae]

yakimae Clarke, 1941 (Depressaria); Proceedings of the U.S. National Museum 90: 185. Holotype ${ }^{\circ}$, USA, Washington, Yakima Co., Yakima, 18 May 1931, F. P. Dean. USNM Type No. 52073, USNM Genitalia Slide No. 611. [Elachistidae: Depressariinae]

yuccasella Busck, 1903 (Deoclona); Proceedings of the U.S. National Museum 25: 837. Holotype ${ }^{\star}$, USA, California, Los Angeles Co., r.f. Yucca whipplei, 14 May 1889, Koebele. USNM Type No. 6368. [Deoclonidae] 
zavalla Busck, 1915 (Ethmia); Proceedings of the Entomological Society of Washington 17: 84. Holotype 9 , USA, Texas Zavalla Co., Nueces River, 28 April 1910, F. C. Pratt. USNM Type No. 19231. [Elachistidae: Ethmiinae]

zonaria Clarke, 1964 (Palinorsa); Proceedings of the U.S. National Museum 116: 200. Holotype $\sigma^{*}$, Bolivia, Monte Cristo, Amazones [no date]. USNM Type No. 64993, USNM Genitalia Slide No. 25768. [Oecophoridae: Oecophorinae]

zostera Clarke, 1978 (Muna); Smithsonian Contributions to Zoology 273: 17. Holotype o Chile, Chiloe Island, Dalcahue, 10-12 February 1954, L. E. Peña. USNM Type No. 73697, USNM Genitalia Slide No. 24250, USNM Wing Slide No. 24192. Although there is no type label on the specimen, it is undoubtedly the holotype since Clarke (1978) described the species from a unique male. [Oecophoridae: Oecophorinae]

\section{Acknowledgments}

We thank Harriet Bramble and Bernard Landry for assistance in organizing the type collection; assistance of the latter was supported by a Smithsonian Collection Improvement Grant to J. Brown. The following provided helpful reviews of the manuscript: Klaus Sat-

tler, The Natural History Museum, London, England; Jean-François Landry, Agriculture Canada, Ottawa, Ontraio, Canada; Gary Miller, USDA, Systematic Entomology Laboratory, Beltsville, Maryland; and David Smith, USDA, Systematic Entomology Laboratory, National Museum of Natural History, Washington, D.C.

\section{Literature cited}

Adamski, D. \& Hodges, R.W. (1996). An annotated list of North American Blastobasinae (Lepidoptera: Gelechioidea: Coleophoridae). Proceedings of the Entomological Society of Washington, $98,708-740$.

Brown, J.W. \& Lewis, J.A. (2000) Catalogue of the type specimens of Tortricidae (Lepidoptera) in the collection of the National Museum of Natural History, Smithsonian Institution, Washington, D.C. Proceedings of the Entomological Society of Washington, 102, 1014-1069.

Baldizzone, G. \& Landry, J.-F. (1993) The identity of Coleophora euryaula Meyrick, 1925 and $C$. vigilis Meyrick, 1925 (Lepidoptera, Coleophoridae). Nota lepidopterologica, 16, 125-137.

Clarke, J.F.G. (1971) The Lepidoptera of Rapa Island. Smithsonian Contributions to Zoology, 56, 1282.

Clarke, J.F.G. (1976) Microlepidoptera: Tortricoidea. Insects of Micronesia, 9, 1-144.

Clarke, J.F.G. (1986) Pyralidae and Microlepidoptera of the Marquesas Archipelago. Smithsonian Contributions to Zoology, 416, 1-485.

Diakonoff, A. (1968) Microlepidoptera of the Philippine Islands. Bulletin of the United States National Museum, 257 (1967), 1-484. 
Flint, O.S., Menke, A.S., Thompson, F.C. \& Ward, R.A. (1976) The United States National Entomological Collections. Smithsonian Institution Press, Washington, DC, 47 pp.

Heinrich, C. \& Loftin, U.C. (1944) August Busck 1870-1944 (Obituary). Proceedings of the Entomological Society of Washington, 46, 230-239.

Hodges, R.W. (1962) A revision of the Cosmopterigidae of America north of Mexico, with a definition of the Momphidae and Walshiidae (Lepidoptera: Gelechioidea). Entomologica Americana, (n.s.) $42,1-166$.

Hodges, R.W. (1974) Gelechioidea: Oecophoridae. Moths of America north of Mexico, fasc. 6.2. The Wedge Entomological Research Foundation, Washington, DC, $142 \mathrm{pp}$.

Hodges, R.W. (1978) Gelechioidea: Cosmopterigidae. Moths of America north of Mexico, fasc. 6.1. The Wedge Entomological Research Foundation, Washington, DC, 166 pp.

Hodges, R.W. (1986) Gelechioidea: Gelechiidae (part). Moths of America north of Mexico, fasc. 7.1. The Wedge Entomological Research Foundation, Washington, DC, 195 pp.

Hodges, R.W. (1998) Gelechioidea. In: Kristensen, N. (ed.), Lepidoptera, Moths and Butterflies. Volume 1: Evolution, Systematics, and Biogeography. Handbook of Zoology 4 (35), Arthropoda: Insecta. Walter de Gruyter, Berlin \& New York, pp. 131-158.

Hodges, R.W. (1999) Gelechioidea: Gelechiidae (part). Moths of America north of Mexico, fascicle 7.6. The Wedge Entomological Research Foundation, Washington, DC, $339 \mathrm{pp}$.

Kaila, L. (1995) A revision of the North American Perittia (= Onceroptila), with first Nearctic records of the genus Mendesia (Elachistidae). Journal of the Lepidopterists' Society, 49, 208222.

Kaila, L. (1996) Revision of the Nearctic species of Elachista. I. The tetragonella group (Lepidoptera: Elachistidae). Entomologia Scandinavica, 27, 217-238.

Kaila, L. (1997) Revision of the Nearctic species of Elachista s. 1. II. The argentella group (Lepidoptera, Elachistidae). Acta Zoologica Fennica, 206, 1-93.

Kaila, L. (1999) Revision of the Nearctic species of Elachista s. 1. III. The bifasciella, praelineata, saccharella and freyerella groups (Lepidoptera, Elachistidae). Acta Zoologica Fennica, 211, 1235.

Miller, S. E. \& R. W. Hodges (1990) Primary types of Microlepidoptera in the Museum of Comparative Zoology (with a discursion on V. T. Chambers' work). Bulletin of the Museum of Comparative Zoology, 152, 45-87.

Park, K.-T. (1995) Gelechiidae from Taiwan II. Hypatima and allies, with descriptions of a new genus and five new species (Lepidoptera, Gelechioidea). Tropical Lepidoptera, 6, 67-85.

Park, K.-T. (1999) Lecithoceridae (Lepidoptera) of Taiwan (I): Subfamily Lecithocerinae: Genera Homaloxestis Meyrick and Lecithocera Herrich-Schaeffer. Zoological Studies (Taipei), 38, 238-256.

Park, K.-T. (2001) Two new Dichomeris (Lepidoptera, Gelechiidae) from Taiwan. Insecta Koreana, $18,307-310$.

Park, K.-T. \& Wu, C. (2001) Additional faunistic data and discussions of Lecithoceridae (Lepidoptera) from Sri Lanka, with descriptions of seven new species. Insecta Koreana, 18, 139 152.

Park, K.-T. \& Wu, C. (2003) A revision of the genus Autosticha Meyrick (Lepidoptera, Oecophoridae) in eastern Asia. Insecta Koreana, 20, 195-226.

Powell, J. A. (1973) A systematic monograph of the New World ethmiid moths (Lepidoptera: Gelechioidea). Smithsonian Contributions to Zoology, 120, 1-302.

Wu, C. (1998) Genus Tisis Walker from Malaysia and Indonesia (Lepidoptera: Lecithoceridae), with description of three new species. Zoological Studies, 37, 191-196.

Wu, C. \& Park, K.-T. (1998) Taxonomic review of the family Lecithoceridae (Lepidoptera) from Sri Lanka II. The subfamily Torodorinae: Genera Deltoplastias, Hygroplasta, and Antiochtha. Insecta Koreana, 15, 81-22. 
Wu, C. \& Park, K.-T. (1999) A taxonomic review of the Lecithoceridae (Lepidoptera) in Sri Lanka ZOOTAXA III. The Subfamily Lecithocerinae: Genus Frisilia. Korean Journal of Systematic Zoology, 15, $1-9$. 


\section{zоотAXA Index to genera}

A

Acanthophlebia 17

Aeaea 113, 132, 144, 145

Aerotypia 114

Afeda 25

Agnippe 25, 54

Agonopterix 15, 28, 32, 34, 39, 40, 43, 47, 49, 60, 68, 75, 103, 114, 128, 137

Agonopterys 70

Agonopteryx 25, 29, 36, 79, 118, 136

Alciphanes 36

Aliciana 62

Altiura 87

Alynda 34, 125, 133

Amorbaea 134

Anacampsis 18, 25, 40, 42, 48, 50, 59, 78, 85, 89, 99, 105, 108, 112, 117, 136, 143

Anapatris 33

Anarsia 19, 33, 117

Anateotricha 75

Anchimompha 89

Ancipita 20

Anesychia 66

Aniuta 90, 103

Anoncia 11, 27, 59, 90, 94, 96, 113, 115, 117, 129, 144

Antaeotricha 30, 47, 61, 75, 94

Antiochtha 84

Antispila 87

Aproaerema 41, 76

Arauzona 95

Aristotelia 17, 24, 36, 53, 59, 67, 70, 75, 77, 82, 94, 97, 99, 107, 110, 119

Arla 136

Aroga 12, 17, 147

Arogalea 130

Arotrura 19

Astiarcha 116

Asymphorodes 7, 9, 11, 13, 16, 25, 26, 29, 32, 34, 41, 45, 46, 51, 56, 59, 67, 69, 71, 80, 81, 85, 86, $89,97,99,103,106,111,113,115,120,126,127,131,140,141$

Atasthalistis 45

Atha 141

Athleta 98

Atoponeura 146

Autosticha 81, 86, 91, 135

Auximobasis 36

B

Batrachedra 23, 28, 37, 39, 41, 42, 43, 58, 61, 66, 71, 82, 88, 95, 101, 107, 113, 121, 122, 125, 137, 140

Beltheca 112

Besciva 84 
Blastobasis 35, 52, 64, 65, 71, 80, 84, 96, 104, 136

Borkhausenia 17, 19, 36, 47, 97

Brachmia 68

Brachyloma 43

Butalis 10, 110

C

Callima 97

Calosima 85

Canthonistis 148

Carodista 54, 65, 140, 147

Carolana 64

Catarata 42, 79, 80, 101, 102, 118

Cerconota 30,46

Chaetocampa 41

Chaetochilus 145

Chedra 44, 73

Chelophoba 89

Chionodes $6,8,13,19,22,24,28,30,31,32,34,35,43,44,48,50,51,53,54,57,59,64,65,66$, $70,71,75,76,77,79,80,83,86,89,91,92,94,96,100,103,104,105,107,108,109,111,112$, $114,115,116,117,119,120,121,122,124,125,126,127,128,130,131,133,135,137,138$, $140,143,144,147$

Chrysoclista 64

Chrysonoma 68

Chrysopeleia 118

Coelopoeta 21

Coleophora 6, 10, 20, 52, 53, 76, 78, 92, 119, 120, 138

Coleotechnites 115

Colinita 131

Coptotelia 12, 37, 42, 50, 63, 99, 108, 110, 136

Corita 13

Cosmopterix 33, 55, 64, 99, 125, 129

Cosmopteryx 35, 56, 58, 74, 81, 93, 98, 145

Costoma 23

Cryptolechia 14, 19, 29, 34, 38, 70, 71, 79, 88, 92, 100, 101, 123, 126, 128, 134, 142

D

Darlia 116

Dasycera 53

Decantha 93, 132, 139

Deia 83

Deltoplastis $37,57,78$

Deoclona 148

Depressaria 12, 13, 14, 18, 20, 22, 23, 24, 29, 38, 42, 48, 50, 57, 60, 62, 76, 81, 87, 95, 96, 99, 105, $109,118,122,124,125,127,138,147,148$

Dichomeris 7, 10, 11, 14, 16, 23, 26, 28, 39, 40, 43, 44, 47, 50, 51, 54, 57, 60, 63, 66, 69, 71, 75, 78, $80,83,85,86,90,92,93,94,95,98,102,103,104,108,124,125,128,129,132,134,135,141$, $142,143,144,146,148$

Dinochares 100

Dita 112

GELECHIOIDEA TYPES IN USNM ～(C) 2004 Magnolia Press 
zootaXA Doina 15, 52, 58, 63, 72, 78, 106, 111, 133, 139, 142

510 Dolidiria 16

Donacivola 123

Dorata 10

Doshia 92

Doxa 146

Doxogenes 18, 31, 68

Durrantia 7

Duvita 146

E

Eccedoxa 138

Echinoglossa 141

Ectaga 80

Elachista 6, 7, 9, 10, 11, 13, 16, 26, 29, 32, 38, 43, 47, 49, 63, 65, 66, 75, 80, 83, 84, 86, 91, 93, 98, $100,101,103,107,109,119,122,125,127,133,135,136$

Elaeonoma 8, 94, 134

Eomichla 66

Ephysteris 69, 84

Epicallima 49, 85, 135

Epigritia 67

Epithectis 35, 134

Eraina 137

Eralea 6

Eriphia 38

Eritarbes 65

Eteobalea 51

Ethmia 6, 14, 27, 28, 32, 35, 36, 39, 41, 43, 44, 49, 51, 52, 56, 59, 62, 63, 66, 69, 79, 86, 88, 89, 94, $95,96,97,104,108,110,113,116,117,118,124,126,127,128,133,139,142,143,146,149$

Eubolepia 15

Euchionodes 139

Euclemensia 125

Eucleodora 36

Eucordylea 50

Eumimographe 41, 84

Eupragia 22, 70

Evagora 46

Evippe 115

Exotelia 15

F

Falculina 23, 76

Filatima $8,11,24,42,64,101,102,110,113,116,122,130,131,141,143,144,146$

Filinota $68,110,121$

Fortinea 20

Frisilia 14, 32, 48, 97, 128, 137, 141, 142

Frumenta 130

G

Galechia 125 
Galtica 145

Gelechia 5, 6, 11, 12, 17, 18, 20, 22, 23, 24, 25, 31, 32, 36, 37, 40, 43, 44, 45, 48, 49, 53, 57, 58, 59, $60,61,62,63,64,65,69,72,73,74,77,78,79,80,81,82,83,84,86,87,94,95,96,97,99$, $100,101,102,104,105,106,107,109,112,113,117,118,119,120,121,122,123,126,128$, $129,131,133,136,139,140,141,142,143,144,148$

Gerdana 30

Glyphidocera 6, 8, 12, 22, 26, 27, 30, 31, 34, 44, 46, 50, 58, 62, 63, 66, 70, 76, 85, 92, 95, 121, 124 , $127,130,145,148$

Gnorimoschema 10, 18, 19, 20, 21, 22, 23, 28, 32, 33, 39, 49, 56, 58, 61, 62, 65, 67, 68, 70, 76, 78, $86,90,93,110,111,116,119,124,126,129,131,134,136,137,142,147$

Gonada 28, 55

Gonionota 9, 13, 17, 26, 33, 37, 38, 45, 47, 52, 54, 57, 61, 67, 70, 71, 73, 91, 104, 106, 109, 112 , $115,126,131,139$

Gonioterma 14, 37, 51, 54, 62, 72, 73, 92, 122, 132, 146

H

Halolaguna 104

Hamadera 20

Harpalyce 10, 30

Hasta 17

Helcystogramma 49, 67

Helice 105

Herlinda 55, 74, 103, 111

Hilarographa 55

Himmacia 132

Holcocera 15, 20, 22, 38, 40, 48, 61, 80, 96, 102, 106, 129, 139, 145

Homaledra 68, 77

Homaloxestis 10, 21, 64, 77, 134

Homoeoprepes 56, 135

Hygroplasta 29, 91, 94, 100, 103, 117, 143

Hypatima 7, 47, 124

Hypatopa 41

Hypercallia 27, 48, 68, 101

Hyperochtha 7, 135

Hyphypena 135

Hypodrasia 8

Hyponomeuta 84

I

Irenia 42,82

Iressa 92,98

Isophrictis 8, 44, 123

Issikiopteryx 33, 135

Ithome 49, 79, 129

K

Kalocyrma 52, 104

L

Laverna 10, 17, 31, 63, 93, 133

GELECHIOIDEA TYPES IN USNM @ 2004 Magnolia Press 
zоOTAXA Lecithocera $7,19,25,43,48,72,81,86,91,106,114,115,136,137$

510 Lelita 7

Lethata $27,47,56,60,79,87,95,123$

Leucophryne 140

Lipatia 41

Lita $45,62,72,105,120,129,137,144$

Lycophantis 50

M

Maesara 61

Malthildana 58

Martyrhilda 75, 97, 131

Martyringa 120

Mastema 102

Melanocinclis 63, 130, 145

Mendesia 91, 127

Menesta 89, 139

Mesoptycha 67

Metathrinca 36, 75, 110

Metopleura 115

Mompha 26, 30, 36, 51, 74, 96, 108, 118, 130, 132

Moriloma 106

Mothonica 77, 102

Muna 149

$\mathrm{N}$

Nanodacna 73

Nealyda 77, 98, 112, 113

Neda 114

Nedenia 121

Neodactylota 22

Nepotula 126

Nosphistica 61

Nothris 35,141

Numata 25

O

Odites 15, 109

Odonna 107, 148

Oecophora 123

Oestomorpha 12

Onceroptila 52

Osmarina 17

$\mathbf{P}$

Palinorsa 7, 149

Palintropa 109

Paltodora 15, 46, 87, 108

Paralechia 28

Paralida 140 
Parasia 16

Paraspistis 105

Parastega 42, 68, 140

Pavolechia 16

Peleopoda 39, 71, 74, 88, 100, 120

Perimede 14, 34, 52, 53, 64, 88, 107

Periploca 18, 45, 69, 70, 74, 76, 77, 98, 103, 121, 130

Perittia 35

Perzelia 16

Petrochroa 46, 134

Phaetusa 114

Philharmonia 54, 73

Philobota 84

Philomusea 90

Phthorimaea 72, 92

Pitycona 20

Pleurota 83

Polyhymno 6

Proclesis 53

Profilinota 111

Promenesta 83, 88

Prostomeus 27

Psacaphora 29, 49, 107, 118

Psecadia 11, 44, 101, 114, 147

Pseudarla 93

Pseuderotis 29

Pseudethmia 117

Pseudochelaria 147

Pseudotelphusa 72

Psilocorsis 30, 41, 55, 57

Psoricoptera 16, 69

Psorosticha 90, 97

Ptilostonychia 114

Ptycerata 27

Pyramidobela 137

Q

Quassitagma 78

$\mathbf{R}$

Recurvaria 12, 37, 40, 50, 53, 57, 74, 76, 98, 118, 138

Retha 123

Revonda 53

Rhindoma 123

$S$

Sathrobrota 21

Scaeothyris 117

Scythris 9, 30, 72, 87, 101

Semioscopis 7, 21, 73, 88, 89, 91 
ZOOTAXA Semolina 82

510 Setiostoma 56

Siskiwitia $12,55,79$

Sophronia 116, 123

Sorhagenia 23, 40, 42, 111

Stagmatophora 148

Stathmopoda 18, 109

Stenoma 8, 9, 14, 22, 26, 33, 35, 37, 40, 44, 46, 47, 54, 55, 56, 59, 60, 65, 66, 73, 74, 77, 78, 82, 83, $85,87,88,90,93,94,98,99,102,104,105,108,110,111,112,114,119,123,124,128,130$, $132,133,134,138,139,140,142,144,146,148$

Stilbosis 83

Swezeyula 84

Symmoca 100

Synallagma 27

$\mathbf{T}$

Tabernillaia 52

Talitha 15

Tamarrha 25

Tanyzancla 13

Teladoma $65,67,72$

Telphusa 6, 21, 24, 33, 88, 89, 101, 102, 144

Teratomorpha 33

Teresita 75

Theisoa 24, 95

Thioscelis 147

Thymiatris 18

Tildenia 62

Timocratica 10, 60, 67, 81, 90, 148

Timyra 15, 31, 35, 103, 132

Tisis 21, 26, 113, 124

Tocasta 116

Tonica 35

Torodora 11, 55, 113, 145

Tosca 114

Trichotaphe 9, 22, 38, 56, 74, 81, 82, 90, 128, 141, 147

Triclonella 54, 109, 127, 145

Trypanisma 55

U

Untomia 143

Urangela 119

Utilia 55, 58

V

Valentinia 58, 119, 121

W

Walshia 11, 50, 58 
Zelosyne 115

Zetesima 79, 115, 138

zoorax 


\section{About the authors}

John W. Brown is a Research Entomologist with the Systematic Entomology Laboratory, USDA, and is stationed at the U.S. National Museum of Natural History, Washington, DC. Although his research focuses primarily on Tortricidae, he is responsible for maintenance and enhancement of the Gelechioidea collection at the National Museum.

David Adamski is a Support Scientist with the Systematic Entomology Laboratory and a Research Associate of the U.S. National Museum of Natural History. He has been with USDA for nearly 15 years in various capacities. His research focuses primarily on the systematics of Blastobasinae.

Ronald W. Hodges is widely recognized as the leading authority on Gelechioidea. Although he retired from a long and productive career with the USDA Systematic Entomology Laboratory in 1997, he has remained extremely active in the field. He is the Executive Director of the Wedge Entomological Foundation which publishes the monographic series "The Moths of North America" (a.k.a. the "Moths of America North of Mexico").

Stephen M. Bahr, II is an undergraduate student at Texas A\&M University. He was a intern in the Entomology Department at the U.S. National Museum during the summer of 2003, during which time he made a significant contribution to the catalog by locating type specimens in the main collection and by tracking down references of original descriptions. 